FZJ-IKP(TH)-2003-16

\title{
Meson production in nucleon-nucleon collisions close to the threshold
}

\author{
C. Hanhart \\ Institut für Kernphysik, Forschungszentrum Jülich GmbH, \\ D-52425 Jülich, Germany
}

\begin{abstract}
The last decade has witnessed great experimental progress that has led to measurements of near threshold cross sections - polarized as well as unpolarized - of high accuracy for various inelastic nucleon-nucleon collision channels. These data, naturally, pose challenges to theorists to develop methods by which they can be understood and explained in commensurate detail.

In this work we review the status of the present theoretical understanding of this class of reactions with special emphasis on model-independent methods. We discuss in detail not only the many observables involved in the reactions, but also the physical questions that can be addressed by studying them in the various reaction channels. The special advantages of nucleon-nucleon induced reactions are stressed. Foremost among these is the use of the initial and final states as a spin/isospin filter. This opens, for example, a window into the spin dependence of the hyperon-nucleon interaction and the dynamics of the light scalar mesons.
\end{abstract}




\section{Contents}

1 Introduction 1

$\begin{array}{lll}1.1 & \text { Strong Interactions at low and medium Energies } & 1\end{array}$

1.2 Theoretical approaches to the reaction $N N \rightarrow B_{1} B_{2} x \quad 4$

1.3 Specific aspects of hadronic meson production close to the threshold 8

$\begin{array}{lll}1.4 & \text { Remarks on the production operator } & 10\end{array}$

$\begin{array}{ll}\text { 1.5 The role of different isospin channels } & 11\end{array}$

$\begin{array}{lll}1.6 & \text { Selection Rules for } N N \rightarrow N N x & 13\end{array}$

$\begin{array}{lll}2 & \text { The final state interaction } & 17\end{array}$

$\begin{array}{lll}2.1 & \text { Heuristic approach } & 17\end{array}$

$\begin{array}{lll}2.2 & \text { Dispersion theoretical approach } & 22\end{array}$

$3 \quad$ The initial state interaction 31

4 Observables 33

$\begin{array}{lll}4.1 & \text { Unpolarized observables } & 35\end{array}$

$\begin{array}{lll}4.2 & \text { Spin dependent observables } & 41\end{array}$

$\begin{array}{lll}4.3 & \text { General structure of the amplitudes } & 50\end{array}$

$\begin{array}{lll}4.4 & \text { Spin Cross Sections } & 60\end{array}$

$\begin{array}{ll}\text { 4.5 Status of Experiment } & 63\end{array}$

$5 \quad$ Symmetries and their violation $\quad 65$

$\begin{array}{lll}5.1 & \text { Investigation of charge symmetry breaking (CSB) } & 65\end{array}$

$\begin{array}{lll}6 & \text { The reaction } N N \rightarrow N N \pi & 69\end{array}$

$\begin{array}{lll}6.1 & \text { Some History } & 69\end{array}$ 
6.3 Chiral perturbation theory

6.4 On the significance of off-shell effects

6.5 Lessons and outlook

7.4 Associated strangeness production or the hyperon-nucleon interaction from production reactions

$\begin{array}{llr}\text { A Kinematical variables } & 124\end{array}$

$\begin{array}{ll}\text { B Collection of useful formulas } & 126\end{array}$

$\begin{array}{lll}\text { B.1 Definition of Coordinate system } & 126\end{array}$

$\begin{array}{llr}\text { B.2 Spin traces } & 127\end{array}$

$\begin{array}{lll}\text { C Partial wave expansion } & 127\end{array}$

D On the non-factorization of a strong final state interaction $\quad 130$ 
E Chiral Counting for Pedestrians

E.1 Counting within TOPT

133

E.2 Counting within the covariant scheme

134

References

144 


\section{Introduction}

\subsection{Strong Interactions at low and medium Energies}

Quantum Chromo Dynamics (QCD), as the well-accepted theory of the Strong Interaction, unfolds its impressive predictive power in particular at high energies. However, with decreasing momentum transfer the mathematical structure of QCD becomes increasingly complicated because the perturbative expansion in the coupling constant no longer converges. The fact that particles carrying strong charge (color) have never been observed in an isolated state is just one prominent example of the non-perturbative character of QCD at low energy. Although a large amount of new data is available from measurements with electromagnetic probes (e.g., from MAMI at Mainz, ELSA at Bonn, and JLAB at Newport News), there is still much to be learned about the physics with hadronic probes at intermediate energies, comprising the investigation of production, decay, and interaction of hadrons. An important class of experiments in this context is meson production in nucleon-nucleon, nucleon-nucleus, and nucleus-nucleus collisions close to the production thresholds. Recently, two reviews on this subject $[1,2]$ were published, however both had their main emphasis on the experimental aspects. In this report we will focus on recent theoretical developments and insights. In addition in neither of these reviews was the potential of using polarization - which will be the main emphasis of this work-discussed in detail.

It is interesting to ask a priori what physics questions can be addressed with the production of various mesons near threshold in $p p, p d$, and $d d$ collisions with stored and extracted polarized beams. We therefore present here a brief

list, naturally influenced by the personal taste of the author, that is not aimed at completeness, but more at giving the reader a flavor of the potential of meson production reaction in nucleonic collisions for gaining insight into the strong interaction physics at intermediate energies.

- Final state interactions: In practice it is difficult to prepare secondary beams of unstable particles with sufficient accuracy and intensity that allow to 
study experimentally the scattering of those particles off nucleons. Here production reactions are an attractive alternative. One prominent example in this context is the hyperon-nucleon interaction that can be studied in the reaction $p p \rightarrow p Y K$, where $Y$ denotes a hyperon $(\Lambda, \Sigma$ etc.) and $K$ denotes a kaon. From the invariant mass distributions of the $Y N$ system, information about the on-shell $Y N$ interaction can be extracted. We shall return to this point in sec. 2.2 .

- Baryon resonances in a nuclear environment: By design, in nucleon-nucleon and nucleon-nucleus collisions we study systems with baryon number larger than 1. This allows to study particular resonances in the presence of other baryons and excited through various exchanged particles. One example is the $N^{*}(1535)$, which is clearly visible as a bump in any $\eta$ production cross section on a single nucleon. A lot is known about this resonance already, however, many new questions can be answered by a detailed study of the reaction $N N \rightarrow N N \eta$. In the close-to-threshold regime, kinematics and the conservation of total angular momentum constrains the initial state to only a few partial waves. This, in combination with the dominance of the $N^{*}(1535)$ in the $\eta$ production mechanism, allows for a detailed study of the $N N \rightarrow N^{*} N$ transition potential. This is relevant to understanding the behavior of the $N^{*}$ in any nuclear environment as well as might reveal information on the structure of the resonance. For a detailed discussion we refer to sec. 7.3 .

In hadronic reactions there is additionally a large number of excitation mechanisms for intermediate resonances possible. Besides pseudoscalars and vector mesons, which are more cleanly studied in pion and photon induced reactions, scalar excitations are possible. It was shown recently that, for instance, the Roper resonance is rather easily excited by a scalar source [3], and thus hadronic reactions might be the ideal place to study this controversial resonance [4].

- Charge symmetry breaking (CSB): Having available several possible initial isospin states ( $p p, p n, d d$, etc.), which all have several possible spin states, allows experiments that make the study of symmetry breaking easily accessible. One example is the impact of charge symmetry breaking on the reaction $p n \rightarrow d \pi^{0}$ : it leads to a forward-backward asymmetry in the dif- 
ferential cross section (c.f. section 5 and Refs. [5,6]). It is important to note that in case of the pion production the leading charge symmetry breaking operators are linked to the up-down mass difference.

A forward-backward asymmetry in the reaction $p n \rightarrow d\left(\pi^{0} \eta\right)_{(s-w a v e)}$ should allow extraction of the CSB $f_{0}-a_{0}$ mixing matrix element - a quantity believed to give insights into the structure of the light scalar mesons.

Once the leading symmetry breaking mechanism is identified, one can use the specific signals caused by CSB to extract information on symmetry conserving matrix elements. For details on the idea as well as an applicationextracting information on the existence of mesonic bound states - we refer the reader to Ref. [7].

- Effective field theory in large momentum transfer reactions: Pion production in nucleon-nucleon collisions is still amenable to treatment within chiral perturbation theory - if the expansion is adapted to the large momentum transfer typical of those reactions. This research is still in progress, however once completed it will allow not only to study systematically the CSB pion production mentioned above, but also to pin down the size of three body forces relevant for a quantitative understanding of $p d$ scattering, and to include the dispersive corrections to $\pi d$ scattering in a chiral perturbation theory analysis. The latter is necessary for an accurate extraction of the isoscalar $\pi N$ scattering length from deuteron reactions, which are otherwise difficult to access. This issue will be discussed in chapter 6.3.

The wealth of information comes with the drawback that, apart from rare cases, it is difficult to extract a particular piece of information from the data. For example, resonances and final state interactions modify in a coherent superposition the invariant mass plot. Fortunately, polarization can act as a spin filter and different contributions to the interaction can be singled out because they show up in the angular distributions of different spin combinations. The various polarization observables will be discussed in detail in sec. 4 .

One characteristic feature of meson production in $N N$ collisions at the first glance looks like a disadvantage with respect to a possible theoretical analysis: the large momentum transfer makes it difficult to reliably construct the production operator. However, as we will discuss in detail, due to this the pro- 


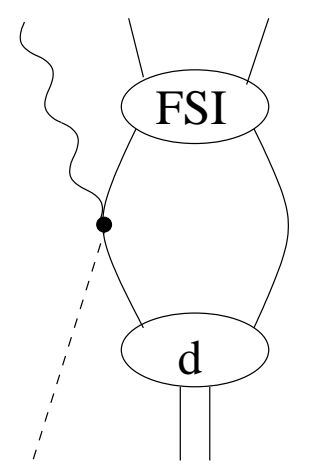

a)

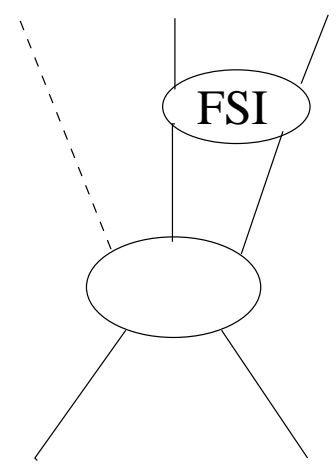

b)

Fig. 1. Comarison of two different reactions that can be used to extract parameters of a strong final state interaction. Shown is a typical low momentum transfer reaction (diagram a), e.g., $K^{-} d \rightarrow \gamma \Lambda n$ ) and a large momentum transfer reaction (diagram b), e.g., $\left.p p \rightarrow p K^{+} \Lambda\right)$.

duction operator is largely independent of the relative energy of a particular particle pair in the final state ${ }^{1}$ if we stay in the regime of small invariant masses. Because of this, dispersion relations can be used to extract low energy scattering parameters of the final state interaction and at the same time give a model-independent error estimate. In contrast, many reactions dominated by one-body currents that are characterized by small momentum transfers (c.f. Fig. 1). This makes a quantitatively controlled, model-independent analysis difficult. For example, it was shown in Refs. [8,9] that value for the neutronneutron $(\Lambda N)$ scattering length extracted from $\pi^{-} d \rightarrow \gamma n n\left(K^{-} d \rightarrow \gamma \Lambda n\right)$, where the initial state is in an atomic bound state, is sensitive to the shortrange behavior of the baryon-baryon interaction used ${ }^{2}$.

\subsection{Theoretical approaches to the reaction $N N \rightarrow B_{1} B_{2} x$}

In this chapter we will briefly describe the theoretical developments for the reactions $N N \rightarrow B_{1} B_{2} x$ close to the threshold, where the $B_{i}$ denote the two

$\overline{1}$ If there are resonances near by this statement no longer true. See the discussion in sec. 4.

2 This implies that meson exchange currents should contribute at the same order of magnitude (c.f. discussion in sec. 6.4). 
outgoing baryons (either $N N$ or, e.g., in case of the production of a strange meson $Y N$, where $Y$ denotes a hyperon).

The development of theoretical models especially for the reaction $N N \rightarrow$ $N N x$ has a long history. For a review of earlier works we refer the reader to the book by H. Garcilazo and T. Mizutani [10]. Most of the models can be put into one of two classes: namely those that use the distorted wave Born approximation, where a production operator that is constructed within some perturbative scheme is convoluted with nucleonic wavefunctions, and truly non-perturbative approaches, where integral equations are solved for the full $(N N, N N x)$ coupled-channel problem.

We start this section with a brief review of the second class. The obvious advantage of this kind of approach is that no truncation scheme is required with respect to multiple rescattering. This is a precondition to truly preserve three-body unitarity [11-13]. However, as we will see, this approach is technically rather involved and there is significant progress still to be made that will allow for quantitative predictions, especially for reactions with unbound few-nucleon systems in the initial and final state.

One obvious complication is to preserve the requirements of chiral symmetry, which are very important - at least for the reaction $N N \rightarrow N N \pi$ close to the threshold, as will be discussed in the following sections, since in its non linear realization it is necessary to consistently take into account one--, two- and more pion vertices as soon as loops are considered. Naturally, this can only be done within a truncation scheme that is consistent with chiral symmetry. As a possible solution, in Ref. [14] it was suggested to change to a linear representation, as given, e.g., by the linear sigma model. To our knowledge this area is not yet very well developed.

All the calculations so far carried out for pion ${ }^{3}$ production within the full $(N N, N N x)$ coupled-channel system, where performed within time ordered perturbation theory. Within this scheme, one way to impose unitarity is it to

$\overline{3 \text { To be }}$ concrete in this section we will talk about pions only. They are, in fact, the only mesons that have so far been studied with integral equation approaches. 


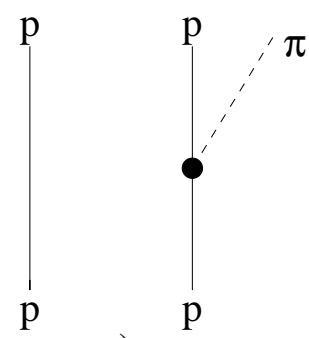

a)

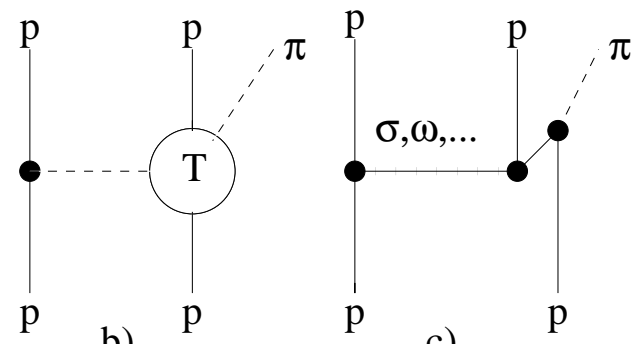

c)

Fig. 2. Some possible contributions to the production operator for neutral pion production in pp collisions. Solid lines are nucleons, dashed lines are pions.

demand, that the number of pions in any given intermediate state should not to exceed a given number. This, in combination with that there is no particle number conservation, implies the fact, that the $\pi N N$ coupling constant generated by the equations at some intermediate time depends on the number of pions in flight at that time. The same is true for the renormalization of the nucleon pole. Put to the extreme: in the nuclear matter limit the dressed pion nucleon coupling vanishes [15] (see also the discussion in Ref. [16]). Another problem inherently present in time-ordered formalisms was pointed out by Jennings [17]: namely that it is impossible simultaneously to fulfill the Pauli-Principle and the unitarity condition within these integral equations. A consequence of this is a wrong sign for $T_{20}$ for elastic $\pi d$ scattering at backward angles [18]. Both of these problems can be avoided within a covariant formulation of the integral equations as is given in Refs. [19,20]. Unfortunately, not only are those equations rather involved, they also need covariant $T$ matrices for both subsystems. Although these exist (e.g., Ref. [21] for the $\pi N$ system and Ref. [22] for the $N N$ system), they were not constructed consistently. Also the three dimensional convolution approach of Refs. [23,16] does not suffer from the above mentioned problems, but also within this scheme so far no results for the $(N N, N N x)$ were published.

On the other hand there are the approaches that treat the production operator perturbatively (typically diagrams of the type shown in Fig. 2 are considered), while including the nucleon-nucleon or, more generally, the baryon-baryon interaction in the final and initial state non-perturbatively. Therefore in this 
formalism three-body unitarity can be achieved only perturbatively, however, this formalism at least fulfills the minimal requirement: to take fully into account the non-perturbative nature of the $N N$ interaction. This is indeed necessary, as was confirmed experimentally, since a proper inclusion of the $N N$ final state interaction is required to describe the energy dependence of the reaction $p p \rightarrow p p X[1,2]$. A production operator that is constructed perturbatively allows in addition to treat the interactions between the subsystems without approximation and consistent with chiral symmetry (c.f. discussion in sec. 6.3). A priori, it is unclear according to what rules the production operator should be constructed and only a comparison with experiment can tell if the approach is appropriate or not. There is, however, one observation in favor of a perturbative treatment of the production operator: in the case of pion production, effective field theory methods can be applied to show that as long as there is a meson exchange current in leading order it dominates the low energy amplitude. Loops undergo strong cancellations and thus might well be neglected. If, however, there is no meson exchange current at leading order (as is the case for the reaction $p p \rightarrow p p \pi^{0}$ ) the situation is significantly more involved and loops might well be significant ${ }^{4}$. This issue will be discussed in detail in sec. 6 . In this introductory chapter we only wish to stress, that to our present knowledge the case of $\pi^{0}$ seems to be an exception and, in addition to the reaction $p p \rightarrow p n \pi^{+}$all heavy meson production cross sections close to the threshold are indeed dominated by meson exchange currents ${ }^{5}$. We must add, however, that the of heavier mesons can also give a significant contribution.

An approach that lies somewhere between those that solve the integral equations for the whole $N N \rightarrow N N \pi$ system and those that treat the production operator perturbatively is that of Refs. [22,24,25]. These works document the attempt to extend straight-forwardly what is known about the phenomenology of nucleon-nucleon scattering (see for example [26]) to energies above the pion threshold. The three-body singularities stemming from both the energy dependent pion exchange as well as the nucleon and Delta self energies were

\footnotetext{
4 Note, however, even then a perturbative treatment of the production operator is still justified.

5 Here this phrase is to be understood to include resonance excitations as well.
} 
taken into account. Via the optical theorem the pion production cross sections can then be calculated in a straight forward way. It turned out, however, that the models could not describe the close to threshold data. The reasons for this will become clear in chapter 6 . In sec. 6.5 we will discuss how the recent progress in our understanding of pion production shows what is needed in order to improve, for example, the work of Ref. [24].

The construction of theoretical models for the production of mesons heavier than the pion started only recently, after the accelerator COSY came into operation. Most of these models are one meson exchange models. Those approaches will be described to some extend in section 7.2 .

\subsection{Specific aspects of hadronic meson production close to the threshold}

In the near-threshold regime the available phase space changes very quickly. Thus, especially when comparing different reactions, an appropriate measure of the energy with respect to that particular threshold is required. In pion production traditionally the variable $\eta$, defined as the maximum pion momentum in units of the pion mass, is used to measure the excess above threshold. For all heavier mesons the so-called excess energy $Q$, defined as

$$
Q=\sqrt{s}-\sqrt{s^{(t h r e s)}}
$$

is normally used. In Appendix A we give the explicit formulas that relate $Q$ to $\eta$. It is useful to understand the physical meaning of the two different quantities: $Q$ directly gives the energy available for the final state. The interpretation of $\eta$ is somewhat more involved. In a non-relativistic, semiclassical picture the maximum angular momentum allowed can be estimated via the relation $l_{\text {max }} \simeq R q^{\prime}$, where $q^{\prime}$ denotes the typical momentum of the corresponding particle and $R$ is a measure of the range of forces. If we identify $R$ with the Compton wavelength of the meson of mass $m_{x}$, we find $l_{\max } \simeq q^{\prime} / m_{x} \simeq \eta$. This interpretation was given for example in Refs. [27,28].

When trying to compare the cross sections of reactions with different final states one has to choose carefully the variable that is used for the energy. In 


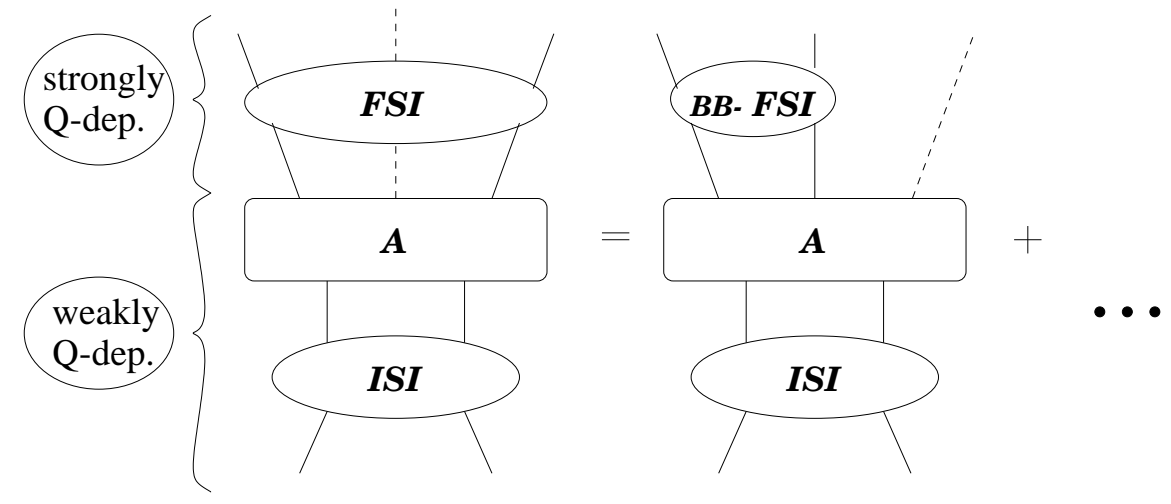

Fig. 3. Sketch of the production reaction showing the initial state interaction amongst the two nucleons (ISI), the final state interaction of all outgoing particles (FSI) as well as the production operator $A$ as defined in the text. The left diagram indicates the complexity possible, whereas the right diagram shows the first and potentially leading term for the FSI only.

Ref. [29] it is shown that the relative strength of the total cross sections for $p p \rightarrow p p \pi^{0}, p p \rightarrow p p \eta$ and $p p \rightarrow p p \eta^{\prime}$ is strikingly different when comparing them at equal $\eta$ or at equal $Q$. Since the dominant final state interaction in all of these reactions is the $p p$ interaction, it appears most appropriate to compare the cross sections at equal $Q$, for then at any given excess energy the impact of the final state interaction is equal for all reactions, which is not the case for equal values of $\eta$. In Ref. [30] a different energy variable was suggested, namely the phase space volume.

In order to produce a meson in nucleon-nucleon collisions the kinetic energy of the initial particles needs to be sufficiently large to put the outgoing meson on its mass shell. To produce a meson of mass $m_{x}$ an initial momentum larger than the threshold value

$$
\left|\vec{p}_{i}{ }^{(t h r e s)}\right|^{2}=m_{x} M_{N}+\frac{m_{x}^{2}}{4}
$$

is necessary. As long as we stay in a regime close to the production threshold the momenta of all particles in the final state are small and therefore $p_{i}^{(\text {thres })}$ also sets the scale for the typical momentum transfer. In a non-relativistic picture a large momentum transfer translates to a small reaction volume, defined by a size parameter $R \sim 1 / p_{i}$. Thus, already for pion production $R$ is as small as $R \sim 0.5 \mathrm{fm}$ and is getting even smaller for heavier mesons. 
Therefore the two nucleons in the initial state have to approach each other very closely before the production of the meson can happen. It then should come as no surprise that it is important for quantitative predictions to understand the elastic as well as inelastic $N N$ interaction in the initial state. However, since for all reactions we will be looking at the initial energy is significantly larger than the excess energy $Q$, the initial state interaction should at most mildly influence the energy dependence. The energy dependence of the production operator should also be weak, for it should be controlled by the typical momentum transfer, which is significantly larger than the typical outgoing momenta.

On the other hand, in the near-threshold regime all particles in the final state have low relative momenta and thus undergo potentially strong final state interactions that can induce strong energy dependences. The different parts of the matrix element are illustrated in Fig. 3.

In the next subsections we will briefly sketch some properties of meson production in nucleon-nucleon collisions in rather general terms with the emphasis on the gross features. Details, as well as selected results for various reactions, will be presented in the subsequent sections.

\subsection{Remarks on the production operator}

As was mentioned above one of the characteristics of meson production in nucleon-nucleon collisions is the large momentum transfer. This leads to a large momentum mismatch for any one-body operator that might contribute to the production reaction.

This reasoning becomes most transparent when looking at it in real space, as shown in Fig. 4. In case of the one-body operator (panel a) the evaluation of the matrix element involves the convolution of a rapidly oscillating function with a mildly oscillating function, which generally will lead to significant cancellations. If there is a meson exchange present, as sketched in panel b, the cancellations will not be as efficient. Thus one should expect that if there is a meson exchange current possible at leading order, it should dominate the 


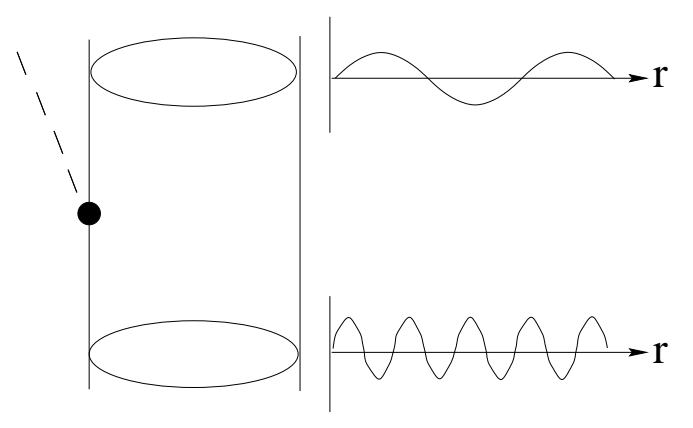

a)

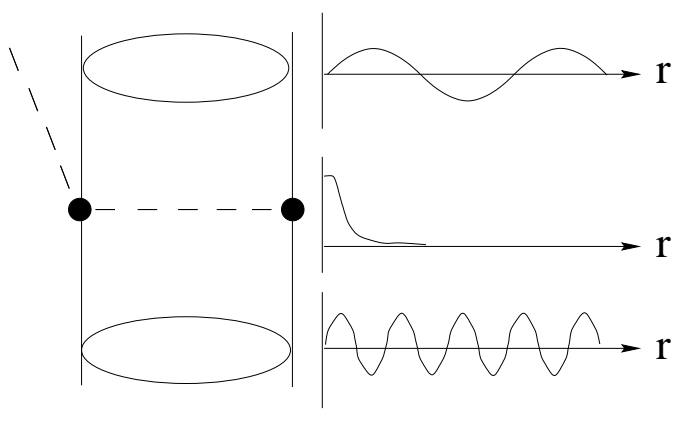

b)

Fig. 4. Illustration of the momentum mismatch. The picture shows why one should a priori expect meson exchange currents to be very important close to the threshold. production process. This picture was confirmed in explicit model calculations (see e.g. Ref. [31]).

As mentioned above one should in any case expect the production operator to be controlled by the large momentum transfer. Thus variations of the individual amplitudes with respect to the total energy should be suppressed by $\left(p^{\prime} / p\right)^{2}$, where $p^{\prime}(p)$ is the relative momentum of an outgoing to particle pair (of the initial nucleons). Therefore it should be negligible close to the production threshold. This will be used for model-independent analyses of the production of heavy mesons and was checked within the meson exchange picture (see discussion in Ref. [32] and sec. 2.2).

\subsection{The role of different isospin channels}

One big advantage of meson production in nucleon-nucleon collisions as well as nucleon-nucleus and nucleus-nucleus collisions is, that besides the spin of the particles there is another internal degree of freedom to be manipulated: the isospin.

Nucleons are isospin-(1/2) particles in an $S U(2)$ doublet (isospin $t=1 / 2$ ) with $t_{3}=+1 / 2$ and $t_{3}=-1 / 2$ for the proton $(p)$ and neutron $(n)$, respectively. Thus a two nucleon pair can be either in an isotriplet state (total isospin $T=1$ with the three possible projections of the total isospin $T_{3}=+1$ for a $p p$ state, $T_{3}=0$ for a $p n$ state and $T_{3}=-1$ for a $n n$ state) or in an isosinglet state 
( $T=0$ with $T_{3}=0$ for a $p n$ state)

Let us first concentrate on the isospin conserving situation. Even then various transitions are possible, depending on whether an isovector or an isoscalar particle is produced. Let us denote the allowed transition amplitudes by $A_{T_{i} T_{f}}$, where $T_{i}\left(T_{f}\right)$ denote the total isospin of the initial (final) $N N$ system [27]. Then, in the case of the production of an isovector, the amplitudes $A_{11}, A_{10}$, and $A_{01}$ are possible. They could be extracted individually from $p p \rightarrow p p x^{0} \propto$ $\left|A_{11}\right|^{2}, p p \rightarrow p n x^{+} \propto\left|A_{11}+A_{10}\right|^{2}$, and $p n \rightarrow p p x^{-} \propto 1 / 2\left|A_{11}+A_{01}\right|^{2}$, where the factor of $1 / 2$ in the latter case stems from the isospin factor of the initial state (we have $|p n\rangle=1 / \sqrt{2}(|10\rangle+|00\rangle)$ in which the isospin states are labeled by both the total isospin as well as their projection). On the other hand, for the production of an isoscalar there are two transitions possible, namely $A_{00}$ and $A_{11}$, where $p p \rightarrow p p x \propto\left|A_{11}\right|^{2}$ and $p n \rightarrow p n x \propto 1 / 2\left|A_{11}+A_{00}\right|^{2}$. In this case the measurement of two different reaction channels allows extraction of all the production amplitudes.

The deuteron is an isoscalar and thus acts as an isospin filter. Accordingly, one finds for the production of any isovector particle $x, A\left(p p \rightarrow d x^{+}\right)=2 A(p n \rightarrow$ $\left.d x^{0}\right)$, as long as isospin is conserved. The consequences of isospin breaking will be discussed in sec. 5 .

The relative strength of the different transition amplitudes proved to provide significant information about the production operator. As an example let us look at the production of an isoscalar particle. If the production operator is dominated by an isovector exchange the corresponding isospin structure of the exchange current is $\hat{O}_{i v}=\left(\vec{\tau}_{1} \cdot \vec{\tau}_{2}\right)$, where $\tau_{i}$ denotes the isospin operator of nucleon $i$, which is clearly distinguished from the structure corresponding to an isoscalar exchange $\hat{O}_{i s}=1$. Thus one finds ${ }^{6}$

${ }^{6}$ The easiest way to see this is to express $\hat{O}_{i v}$ in terms of the Casimir operators of the underlying group:

$$
\vec{\tau}_{1} \cdot \vec{\tau}_{2}=4 \hat{t}_{1} \cdot \hat{t}_{2}=2\left(\hat{T}^{2}-\hat{t}_{1}^{2}-\hat{t}_{2}^{2}\right)
$$

with $<T^{2}>=T(T+1)$ and $<t_{i}^{2}>=3 / 4$. 


$$
\left\langle T T_{3}\left|\hat{O}_{i v}\right| T T_{3}\right\rangle=2 T(T+1)-3=\left\{\begin{aligned}
1 & \text { for } T=1 \\
-3 & \text { for } T=0
\end{aligned}\right.
$$

On the other hand

$$
\left\langle T T_{3}\left|\hat{O}_{i s}\right| T T_{3}\right\rangle=1
$$

For two given $N N$ states a different total isospin implies that also either the total spin or the angular momentum are different (c.f. next section). Therefore, different isospin states can not interfere in the total cross section and one may expect

$$
\frac{\sigma_{t o t}(p n \rightarrow p n x)}{\sigma_{t o t}(p p \rightarrow p p x)} \sim \begin{cases}5 & \text { if isovector exchanges dominate } \\ 1 & \text { if isoscalar exchanges dominate }\end{cases}
$$

For $x=\eta$ a recent measurement at Uppsala [33] gave for this ratio a value of 6.5, clearly indicating a dominance of isovector exchanges. It should be stressed that the estimates given here only hold if effects from initial and final state interactions, the spin dependence of the production operator, as well as all other dynamical effects are completely neglected. However, even in the most detailed studies, the bulk of the ratio stems from the isospin factors [34-36].

\subsection{Selection Rules for $N N \rightarrow N N x$}

In this section we will present the selection rules relevant for nucleon-nucleon induced meson production. Those rules are based on the symmetries of the strong interaction that imply the conservation of parity, total angular momentum and isospin.

A two-nucleon system has to obey the Pauli Principle, which implies $(-)^{L+S+T}=(-1)$ where $L, S$ and $T$ denote the angular momentum, total spin and total isospin of the two nucleon system respectively. In the case of a two proton system, for example, where $T=1, L+S$ needs to be even. Consequently, for $T=1$ all even angular momenta, and therefore all even parity states, are spin singlet $(S=0)$ states and consequently have $J=L$. On the other hand, for $T=0$ states it is the odd parity states that are spin singlets. 
In addition, for a reaction of the type $N N \rightarrow N N x$ we find from parity conservation

$$
(-)^{L}=\pi_{x}(-)^{\left(L^{\prime}+l^{\prime}\right)}
$$

where $\pi_{x}$ denotes the intrinsic parity of particle $x$ and $L, L^{\prime}$ and $l^{\prime}$ denote, respectively, the angular momentum of the incoming two nucleon system, of the outgoing two-nucleon system and of particle $x$ with respect to the outgoing two-nucleon system.

We may now combine the two criteria to write

$$
(-)^{(\Delta S+\Delta T)}=\pi_{x}(-)^{l^{\prime}}
$$

where $\Delta S(\Delta T)$ denotes the change in total (iso)spin when going from the initial to the final $N N$ system.

As an example let us look at the reaction $p n \rightarrow d(\pi \eta)_{s-\text { wave }}$ - a reaction that should provide valuable information on the light scalar resonances (note: the $\pi \eta$ channel is the dominant decay channel of the scalar-isovector meson $a_{0}(980)$, c.f. chapter 7.5). Based on Eq. (9), the near-threshold regime is dominated by the lowest partial waves which in this case would be $S^{\prime}=1$ and $T^{\prime}=0$ (deuteron), together with a relative $s$ wave between the two meson system and the deuteron $\left(l^{\prime}=0\right)$. In addition, the $(\pi \eta)_{s-w a v e}$ system is a scalar-isovector system. Therefore we have to use $\pi_{x}=+, l^{\prime}=0$ and $\Delta T=1$ in Eq. (3), yielding $\Delta S=0$. On the other hand the initial state needs to be of even parity, but even parity $T=1$ states have $S=0$. Thus, for the production of a scalar-isovector state the $s$-wave deuteron with respect to the two meson system is prohibited and, as long as isospin is conserved, this reaction has to have at least one $p$-wave in the final state. On the other hand, for the production of an isoscalar scalar particle, the $s$-wave final state is allowed.

In a straight-forward way the corresponding selection rules for systems with nuclei in the initial and/or final state can be easily derived along the same lines. Bose symmetry, for example, demands a $d d$ system to be symmetric under exchange of the two deuterons, forcing $L+S$ to be even. Therefore the lowest partial waves contributing to $d d \rightarrow \alpha \eta$ are ${ }^{3} P_{0} \rightarrow s$ and ${ }^{5} D_{1} \rightarrow p$.

Another example of selection rules at work in a system other than $N N \rightarrow$ 


\begin{tabular}{|c|c|c|c|c|c|c|c|c|c|}
\hline$L_{N N} l_{x}$ & $(N N)_{i}$ & $(N N)_{f} l^{\prime}$ & $\mathrm{S}$ & $\mathrm{L}$ & $\mathrm{J}$ & $\mathrm{S}^{\prime}$ & $\mathrm{L}^{\prime}$ & j' & l' \\
\hline \multicolumn{10}{|c|}{$\left.\left.N N\right|_{(T=1)} \rightarrow N N\right|_{(T=1)}+$ pseudo-scalar (e.g., $\left.p p \rightarrow p p \pi^{o}\right)$} \\
\hline Ss & ${ }^{3} P_{0}$ & ${ }^{1} S_{0} s$ & 1 & 1 & 0 & 0 & 0 & 0 & 0 \\
\hline $\mathrm{Sp}$ & \multicolumn{9}{|c|}{ not allowed } \\
\hline \multirow[t]{2}{*}{ Ps } & ${ }^{1} S_{0}$ & ${ }^{3} P_{0} s$ & 0 & 0 & 0 & 1 & 1 & 0 & 0 \\
\hline & ${ }^{1} D_{2}$ & ${ }^{3} P_{2} s$ & 0 & 2 & 2 & 1 & 1 & 2 & 0 \\
\hline \multicolumn{10}{|c|}{$\left.\left.N N\right|_{(T=1)} \rightarrow N N\right|_{(T=0)}+$ pseudo-scalar (e.g., part of $\left.p p \rightarrow p n \pi^{+}\right)$} \\
\hline Ss & ${ }^{3} P_{1}$ & ${ }^{3} S_{1} s$ & 1 & 1 & 1 & 1 & 0 & 1 & 0 \\
\hline \multirow[t]{2}{*}{$\mathrm{Sp}$} & ${ }^{1} S_{0}$ & ${ }^{3} S_{1} p$ & 0 & 0 & 0 & 1 & 0 & 1 & 1 \\
\hline & ${ }^{1} D_{2}$ & ${ }^{3} S_{1} p$ & 0 & 2 & 2 & 1 & 0 & 1 & 1 \\
\hline Ps & \multicolumn{9}{|c|}{ not allowed } \\
\hline \multicolumn{10}{|c|}{$\left.\left.N N\right|_{(T=0)} \rightarrow N N\right|_{(T=1)}+$ pseudo-scalar (e.g., part of $\left.p n \rightarrow p p \pi^{-}\right)$} \\
\hline Ss & \multicolumn{9}{|c|}{ not allowed } \\
\hline \multirow[t]{2}{*}{$\mathrm{Sp}$} & ${ }^{3} S_{1}$ & ${ }^{1} S_{0} p$ & 1 & 0 & 1 & 0 & 0 & 0 & 1 \\
\hline & ${ }^{3} D_{1}$ & ${ }^{1} S_{0} p$ & 1 & 2 & 1 & 0 & 0 & 0 & 1 \\
\hline \multirow[t]{2}{*}{ Ps } & ${ }^{3} S_{1}$ & ${ }^{3} P_{1} s$ & 1 & 0 & 1 & 1 & 1 & 1 & 0 \\
\hline & ${ }^{3} D_{J}$ & ${ }^{3} P_{J} s$ & 1 & 2 & $\mathrm{~J}$ & 1 & 1 & $\mathrm{~J}$ & 0 \\
\hline \multicolumn{10}{|c|}{$\left.\left.N N\right|_{(T=0)} \rightarrow N N\right|_{(T=0)}+$ pseudo-scalar (e.g., part of $\left.p n \rightarrow p n \eta\right)$} \\
\hline Ss & ${ }^{1} P_{1}$ & ${ }^{3} S_{1} s$ & 0 & 0 & 0 & 1 & 0 & 1 & 1 \\
\hline \multirow[t]{2}{*}{$\mathrm{Sp}$} & ${ }^{3} S_{1}$ & ${ }^{3} S_{1} p$ & 1 & 0 & 1 & 1 & 0 & 1 & 1 \\
\hline & ${ }^{3} D_{J}$ & ${ }^{3} S_{1} p$ & 1 & 2 & $\mathrm{~J}$ & 1 & 0 & 1 & 1 \\
\hline \multirow[t]{2}{*}{ Ps } & ${ }^{3} S_{1}$ & ${ }^{1} P_{1} s$ & 1 & 0 & 1 & 0 & 1 & 1 & 0 \\
\hline & ${ }^{3} D_{1}$ & ${ }^{1} P_{1} s$ & 1 & 2 & 1 & 0 & 1 & 1 & 0 \\
\hline
\end{tabular}

Table 1

The lowest partial waves for the production of a pseudo-scalar particle in $N N \rightarrow$ $N N x$. 


\begin{tabular}{|c|c|c|c|c|c|c|c|c|c|}
\hline$L_{N N} l_{x}$ & $(N N)_{i}$ & $(N N)_{f} l^{\prime}$ & $\mathrm{S}$ & $\mathrm{L}$ & $\mathrm{J}$ & $S^{\prime}$ & $\mathrm{L}^{\prime}$ & j' & l' \\
\hline \multicolumn{10}{|c|}{$\left.\left.N N\right|_{(T=1)} \rightarrow N N\right|_{(T=1)}+$ scalar (e.g., $\left.p p \rightarrow p p a_{0}^{0}\right)$} \\
\hline Ss & ${ }^{1} S_{0}$ & ${ }^{1} S_{0} s$ & 1 & 0 & 0 & 0 & 0 & 0 & 0 \\
\hline $\mathrm{Sp}$ & ${ }^{3} P_{1}$ & ${ }^{1} S_{0} p$ & 1 & 1 & 1 & 0 & 0 & 0 & 1 \\
\hline \multirow[t]{2}{*}{ Ps } & ${ }^{3} P_{J}$ & ${ }^{3} P_{J} s$ & 1 & 1 & $\mathrm{~J}$ & 1 & 1 & $\mathrm{~J}$ & 0 \\
\hline & ${ }^{3} F_{2}$ & ${ }^{3} P_{2} s$ & 1 & 3 & 2 & 1 & 1 & 2 & 0 \\
\hline \multicolumn{10}{|c|}{$\left.\left.N N\right|_{(T=1)} \rightarrow N N\right|_{(T=0)}+$ scalar (e.g., part of $\left.p p \rightarrow p n a_{0}^{+}\right)$} \\
\hline Ss & \multicolumn{9}{|c|}{ not allowed } \\
\hline \multirow[t]{2}{*}{$\mathrm{Sp}$} & ${ }^{3} P_{J}$ & ${ }^{3} S_{1} p$ & 1 & 1 & $\mathrm{~J}$ & 1 & 0 & 1 & 1 \\
\hline & ${ }^{3} F_{2}$ & ${ }^{3} S_{1} p$ & 1 & 3 & 2 & 1 & 0 & 1 & 1 \\
\hline \multirow[t]{2}{*}{ Ps } & ${ }^{3} P_{J}$ & ${ }^{3} P_{J} s$ & 1 & 1 & $\mathrm{~J}$ & 1 & 1 & $\mathrm{~J}$ & 0 \\
\hline & ${ }^{3} F_{2}$ & ${ }^{3} P_{2} s$ & 1 & 3 & 2 & 1 & 1 & 2 & 0 \\
\hline \multicolumn{10}{|c|}{$\left.\left.N N\right|_{(T=0)} \rightarrow N N\right|_{(T=1)}+$ scalar $\left(\right.$ e.g., part of $\left.p n \rightarrow p p a_{0}^{-}\right)$} \\
\hline Ss & \multicolumn{9}{|c|}{ not allowed } \\
\hline $\mathrm{Sp}$ & ${ }^{1} P_{1}$ & ${ }^{1} S_{0} p$ & 0 & 1 & 1 & 0 & 0 & 0 & 1 \\
\hline Ps & ${ }^{1} P_{1}$ & ${ }^{3} P_{1} s$ & 0 & 1 & 1 & 1 & 1 & 1 & 0 \\
\hline \multicolumn{10}{|c|}{$\left.\left.N N\right|_{(T=0)} \rightarrow N N\right|_{(T=0)}+$ scalar $\left(\right.$ e.g., part of $\left.p n \rightarrow p n f_{0}\right)$} \\
\hline \multirow[t]{2}{*}{ Ss } & ${ }^{3} S_{1}$ & ${ }^{3} S_{1} s$ & 1 & 0 & 1 & 1 & 0 & 1 & 0 \\
\hline & ${ }^{3} D_{1}$ & ${ }^{3} S_{1} s$ & 1 & 2 & 1 & 1 & 0 & 1 & 0 \\
\hline $\mathrm{Sp}$ & ${ }^{1} P_{1}$ & ${ }^{3} S_{1} p$ & 0 & 1 & 1 & 1 & 0 & 1 & 1 \\
\hline Ps & ${ }^{1} P_{1}$ & ${ }^{1} P_{1} s$ & 0 & 1 & 1 & 0 & 1 & 1 & 0 \\
\hline
\end{tabular}

Table 2

The lowest partial waves for the production of a scalar particle in $N N \rightarrow N N x$. 


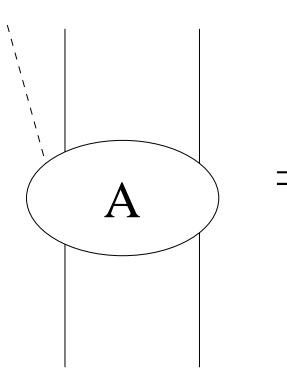

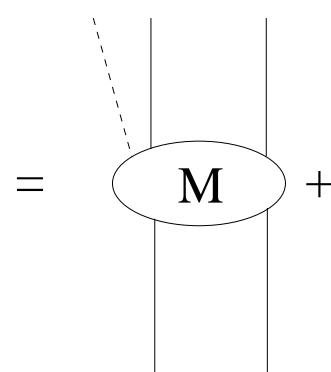

a)

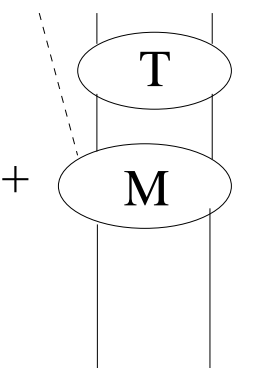

b)

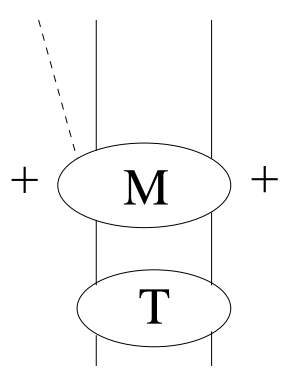

c)

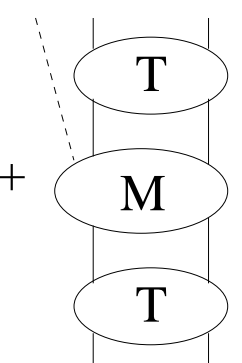

d)

Fig. 5. Diagrammatic presentation of the distorted wave Born approximation. $N N x$ will be given in sec. 4.3.1.

\section{The final state interaction}

The role of final state interactions in production reactions has been discussed in the literature for a long time and at various levels of sophistication. Extensive discussions can be found in Refs. [37,38]. For more recent discussions focusing on the production of mesons in nucleon-nucleon collisions we refer to Refs. [39,40,32]. In this section we will approach the question of the role of the final state interactions from two approaches: first we will present a heuristic approach that should make the physics clear, but has only limited quantitative applicability; then, using dispersion integrals, quantitative expressions will be derived, but these are less transparent. This investigation will show under what circumstances scattering parameters can be extracted from high momentum transfer production reactions, and will address questions about the accuracy of this extraction.

\subsection{Heuristic approach}

As early as 1952 Watson pointed out the circumstances under which one would expect the final state interaction to strongly modify the energy dependence of the total production cross-section $N N \rightarrow N N x$ [41]. His rather convincing argument went as follows: assume in contrast to the production reaction the pion absorption on a free two nucleon pair. Due to time reversal invariance the matrix elements for the two reactions are equal. Based on the observation that 
the absorption takes place in a small volume, it is obvious that the reaction is a lot more probable if, in a first step, the two nucleons come together rather closely due to their attractive interaction and that this significantly more compact object then absorbs the pion. In this picture the influence of the two-nucleon system on the energy dependence of the cross section is very natural. Based on this argument, one should expect ${ }^{7}$

$$
\sigma_{N N \rightarrow N N x}(\eta) \propto \int_{0}^{m_{x} \eta} d^{3} q^{\prime} p^{\prime} d \Omega_{p^{\prime}}\left|\Phi_{x}^{\dagger}\left(q^{\prime}\right) \Psi_{N N}\left(p^{\prime}\right)\right|^{2}
$$

where $q^{\prime}\left(p^{\prime}\right)$ denotes the momentum of the outgoing meson (the relative momentum of the outgoing two nucleon pair) and $d^{3} q^{\prime}=d q^{\prime} d \Omega_{q^{\prime}}$; energy conservation implies $p^{\prime 2}=M_{N}\left(E-\omega_{q^{\prime}}\right)$, where $\omega_{q^{\prime}}$ denotes the energy of the meson. In Eq. (4) $\Psi\left(p^{\prime}, p^{\prime}\right)$ is the $N N$ wavefunction at the energy, $2 E\left(p^{\prime}\right)$, of the final $N N$ subsystem, where $E\left(p^{\prime}\right) \equiv \frac{p^{\prime 2}}{2 M_{N}}$ and $\Phi_{x}$ denotes the wavefunction for the outgoing meson. If we now neglect the impact of the meson-nucleon interaction on the energy dependence, the meson wave function is given by a plane wave. For its component in the $l$-th partial wave we get [42]

$$
\Phi_{x}^{l}\left(q^{\prime}\right) \propto j_{l}\left(q^{\prime} R\right) \simeq q^{\prime l}
$$

where $j_{l}\left(q^{\prime} R\right)$ denotes the $l$ th Bessel function. The term $q^{\prime l}$ is the first term in an expansion of $q^{\prime} R$, where $R$ represents the range of forces. Therefore, Eq. (5) should appropriately describe the $q^{\prime}$ dependence of the corresponding partial wave amplitude as long as $\left(q^{\prime} R\right) \ll 1$. In addition, when the final state interaction is very strong and shows a strong energy dependence (as, for example, the nucleon-nucleon interaction in the ${ }^{1} S_{0}$ for small relative energies), we may replace the $N N$ wave function by the on-shell $T$ matrix, which can be written in the $L$-th partial wave

$$
T^{L}\left(E_{p^{\prime}}\right)=T_{0}^{L} e^{i \delta_{L}\left(E_{p^{\prime}}\right)} \frac{\sin \delta_{L}\left(E_{p^{\prime}}\right)}{p^{\prime L+1}}
$$

where $\delta_{L}\left(E_{p^{\prime}}\right)$ denotes the corresponding phase shift as deduced from elastic $N N$ scattering data. In practice this means that we neglect the term with the non-interacting outgoing two nucleon pair (Fig. 5a) und c)) compared to that with the pair interacting (Fig. 5b) and d)). In case of the $N N$ final state

7 Here all final state particles are treated non-relativistically for simplicity. 
interaction this is indeed justified. In general, however, the plane wave piece is not negligible. Fortunately in this case dispersion integrals may be used to fix the relative strength of diagrams $a$ and $b$ of Fig. 5. This will be discussed in detail in the next section.

At low energies the $T$ matrix may be replaced by the first term in the effective range expansion ${ }^{8}$.

$$
p^{2 L+1} \cot \delta_{L}\left(E_{p^{\prime}}\right)=\frac{1}{a_{L}}
$$

where $a_{L}$ denotes the scattering length of the $N N$ interaction in the partial wave characterized by $L$. Thus we can write

$$
\sigma_{N N \rightarrow N N x}^{(L, l)}(\eta) \propto \int_{0}^{m_{x} \eta} d q^{\prime} q^{2 l+2} \frac{p^{2 L+1}}{1+a_{L}^{2} p^{\prime 4 L+2}} .
$$

Since Eq. (5) only holds for $\left(q^{\prime} R\right) \ll 1$, in Eq. (8) the $a_{L}$-term has to be kept in the denominator only if $a_{L} \gg R^{2 L+1}$ In case of the $N N$ interaction, where $R \sim m_{\pi}$ this condition holds for the $S$-waves only. When data for the reaction $p p \rightarrow p p \pi^{0}$ close to threshold became available $[43,44]$ Eq. 8 indeed turned out to give the correct energy dependence of the total cross-section [45]. Eq. (8) contains two very important messages. First of all that a strong final state interaction will significantly change the invariant mass distribution of the corresponding two particle subsystem - the relevant scale parameter is $\left(a_{L} p^{\prime}\right)$. This observation was used in Ref. [46] to extract information on the $\Lambda N$ interaction. We come back to this in section 2 . Secondly that due to the centrifugal barrier higher partial waves are suppressed in the close to threshold regime. As a rough estimate we can take non relativistic kinematics to derive

$$
\sigma_{N N \rightarrow N N x}^{(L, l)}(\eta) \propto\left(\frac{\mu_{N N}}{\mu_{(N N) x}}\right)^{L}\left(m_{x} \eta\right)^{2 l+2 L+4} \propto \mu_{N N}^{L} \mu_{(N N) x}^{l} Q^{l+L+2}
$$

for all those partial waves where there is no strong FSI. Here we used the relation between $Q$ and $\eta$ as given in Eq. (A.4) and $\mu_{N N}=M_{N} / 2$ and $\mu_{(N N) x}=$ $m_{x} /\left(1+m_{x} /\left(2 M_{N}\right)\right)$ denote the reduced masses of the $N N$ or more generally

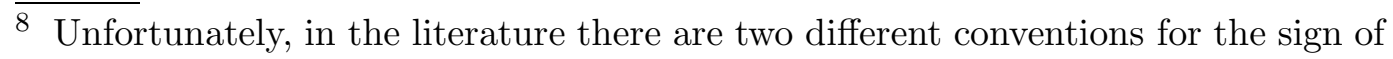
the scattering length. The convention used here is that of Goldberger and Watson [37]; the usual convention for baryon-baryon interactions has a minus sign on the right hand side. 
the baryon-baryon system and of the particle $x$ with respect to the $N N$ system respectively.

Eq. (9) contains two pieces of information: first of all it shows that, for a given value of $Q$, heavier systems are more likely to be in a higher partial. The reason is that their maximum momentum allowed by energy conservation is larger than that of light particles. Secondly it should give a reasonable estimate of the energy dependence of the partial cross sections for all those partial waves where the $N N$ system is in a partial wave higher than the $S-$ wave. In case of the pion production this finding was confirmed recently in Ref. [47] for the reaction $p p \rightarrow p p \pi^{0}$ as is demonstrated in Fig. 6, where the spin cross sections ${ }^{1} \sigma_{0}$ and ${ }^{3} \sigma_{1}$ are shown as a function of $\eta$. We use the notation of Ref. [47] as ${ }^{2 S+1} \sigma_{m}$, where $S(m)$ denotes the total spin (spin projection) of the initial state. For the definition we refer to chapter 4.2. It is easy to show that the lowest partial waves in the final state that contribute to ${ }^{1} \sigma_{0}$ $\left({ }^{3} \sigma_{1}\right)$ are $P s(P p$ and $D s)$, where capital letters denote the relative angular momentum of the two nucleons and the small letters that of the pion with respect to the two-nucleon system. Here we used that pion $d$-waves should be suppressed compared to $N N D$-waves (c.f. Eq. (9)). Accordingly one should expect the $P s$ states to have an energy dependence given by $\eta^{6}$ whereas the $P p$ states should follow an $\eta^{8}$ dependence (c.f. Eq. (9)). The corresponding fits are shown in the Figure as a solid and a dashed line, respectively. The good agreement of these simple forms for the energy dependence of the different partial waves supports the conjecture that neither the initial state interaction nor the production operator and not even the FSI, for partial waves higher than $S$-waves, induce a significant energy dependence beyond the centrifugal barrier.

As we have seen, the energy dependence factorizes from the total amplitude. The situation is somewhat more complicated, however, when it comes to quantitative predictions for the cross section: in the presence of very strong final state interactions it is not possible to separate model independently production operator and final state interaction. The reason for this is that what sets the scale for the size of the matrix element is the convolution integral of the production operator with the final state half off-shell $N N$ T-matrix. This con- 


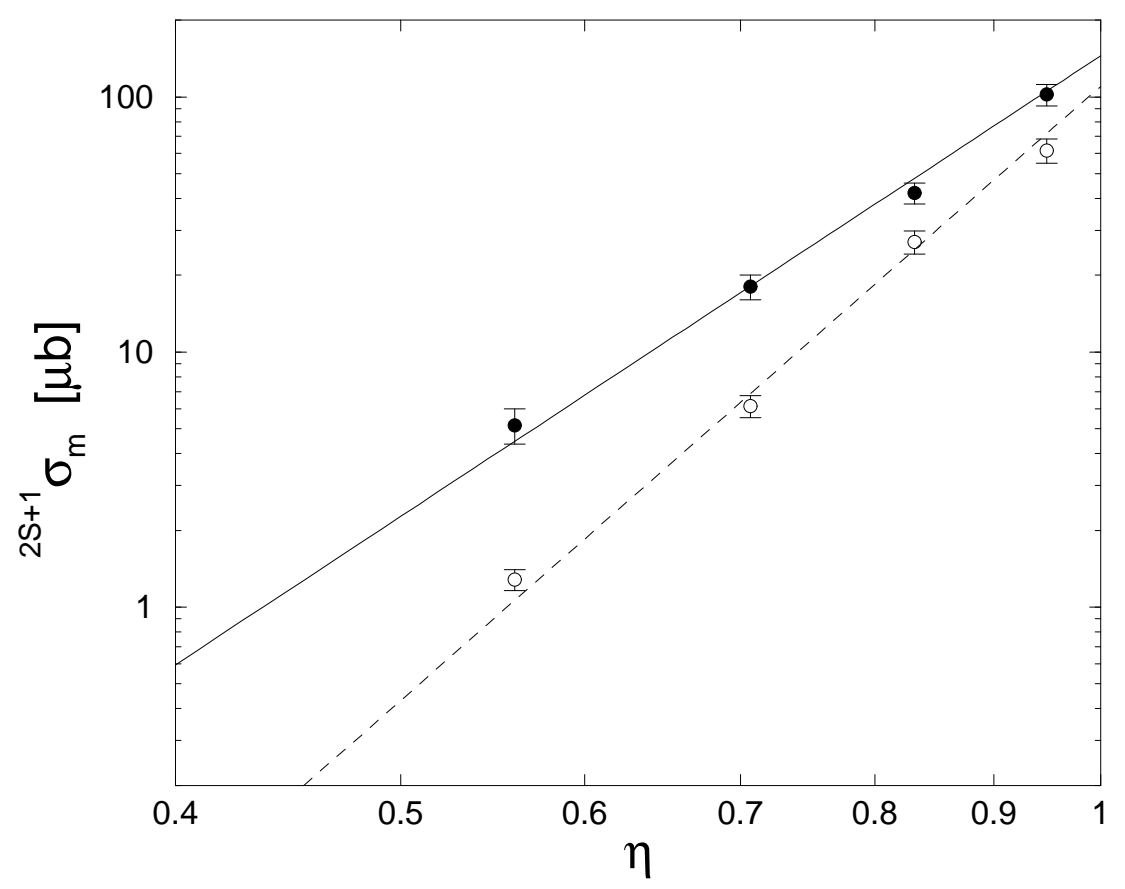

Fig. 6. Energy dependence of two different spin cross sections for the reaction $p p \rightarrow p p \pi^{0}$. The data for ${ }^{1} \sigma_{0}\left({ }^{3} \sigma_{1}\right)$, shown as solid (opaque) dots, are compared to a fit of the form $y=A \eta^{\tau}$ with $\tau=6(\tau=8)$. The data are from table $V$ of Ref. [47] with an additional error of $10 \%$ on the total cross section.

volution consists of a unitarity cut contribution in which the intermediate two nucleons are on their mass shell, and a principal value piece that is sensitive to the off-shell behavior of both the $N N$ T-matrix as well as the production operator. This already shows that it is impossible to separate the production operator from the final state interaction in a model independent way. Since off-shell effects are not observable, a consistent construction of production operator and final state interaction is required to get meaningful results for the observables.

In order to keep the formulas simple so far we have not discussed at all the effect of the Coulomb interaction, although its effect especially on near threshold meson production with two protons in the final state is known to be quite strong [45]. To account for the Coulomb interaction in the formulas given above one should use the Coulomb modified effective range expansion instead of Eq. (7). The corresponding formulas are e.g. given in Ref. [48]. 


\subsection{Dispersion theoretical approach}

The fact that meson production in nucleon-nucleon collisions is a high momentum transfer reaction allows for a rigorous treatment of strong final state interactions within a dispersion theoretical approach ${ }^{9}$. As we will see, in those cases where the effective range of a particular final state interaction is of the order of the scattering length, the modification of the invariant mass spectrum induced by the corresponding final state interaction is no longer proportional to the elastic scattering situation. However, the corrections that arise can be accounted for systematically. In addition to things that are are well known and presented in various text books $[37,38]$, we derive an integral representation for the scattering length in terms of an observable [32]. In addition one gets at the same time an integral representation of the uncertainty of the method. The latter can be estimated using scale arguments or by doing a model calculation. For example, in the case of the hyperon-nucleon interaction the scattering lengths as extracted from the available data on elastic scattering are of the order of a few fermi, but with an uncertainty of a few fermi-especially for the spin singlet scattering length (c.f. discussion in section 7.4). Using the kinematics for associated strangeness production to estimate of the theoretical uncertainty, we find a value of $0.3 \mathrm{fm}$. Thus it is feasible from the theoretical point of view to improve significantly our knowledge of the hyperon-nucleon scattering parameters through an analysis of the reaction $p p \rightarrow p K \Lambda$.

To be definite, we will discuss the reaction $p p \rightarrow p K \Lambda$, however - as should be obvious - the complete discussion also applies to any subsystem with a sufficiently strong interaction. Let us assume the $\Lambda p$ system is in a single partial wave (this can be easily extracted from polarized experiments as will be discussed in sec. 4.3.1) and the third particle - here the kaon - is produced with a definite momentum transfer $t=\left(p_{1}-p_{K}\right)^{2}$, where $p_{1}$ denotes the beam momentum. The full production amplitude may then be written as

\footnotetext{
9 Actually, the formalism applies to all large momentum transfer reactions. In addition to meson production in $N N$ collisions, one may study e.g. $\gamma d \rightarrow B_{1} B_{2}+$ meson. This reaction is also discussed in Refs. [49-54].
} 


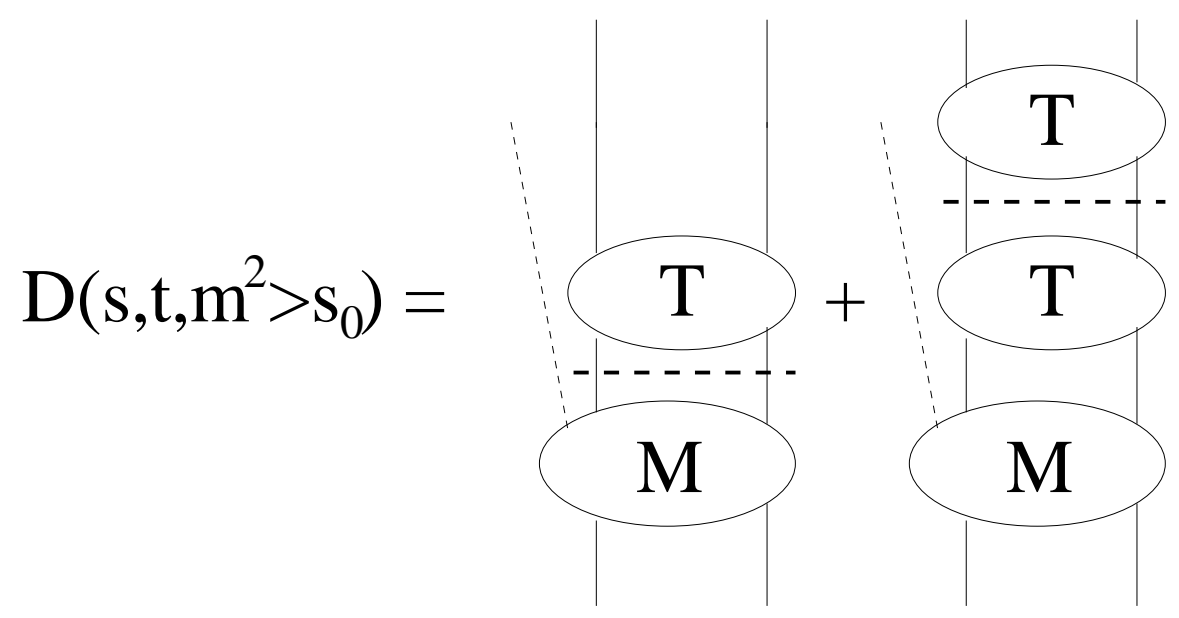

Fig. 7. Illustration of the sources of a discontinuity in the amplitude A from two baryon intermediate states. The dashed horizontal lines indicate the presence of a two baryon unitarity cut (Diagrams with initial state interaction are not shown explicitly (c.f. Fig. 5)).

$$
A\left(s, t, m^{2}\right)=\frac{1}{\pi} \int_{-\infty}^{\tilde{m}^{2}} \frac{D\left(s, t, m^{\prime 2}\right)}{m^{\prime 2}-m^{2}} d m^{\prime 2}+\frac{1}{\pi} \int_{m_{0}^{2}}^{\infty} \frac{D\left(s, t, m^{\prime 2}\right)}{m^{\prime 2}-m^{2}} d m^{\prime 2},
$$

where $m^{2}=\tilde{m}^{2}$ is the lefthand singularity closest to the physical region, $m_{0}=M_{N}+M_{\Lambda}$ corresponds to the first right hand cut, and

$$
D\left(s, t, m^{2}\right)=\frac{1}{2 i}\left(A\left(s, t, m^{2}+i 0\right)-A\left(s, t, m^{2}-i 0\right)\right) .
$$

Note that, in contrast to the representation of the amplitude in case of one open channel only as in $\bar{N} N \rightarrow \pi \pi, D$ is not simply given by the imaginary part of $A$, since the initial state interaction also induces a phase in $A$ (c.f. discussion in the next section). Thus $D$ has to be written explicitly as the discontinuity of $A$ along the appropriate two-particle cut.

The second integral in Eq. (10) gets contributions from the various possible final state interactions, namely $\Lambda K, N K$ and $\Lambda N$, where the latter is the strongest. We may thus neglect the former two for a moment to get, for $m^{2}>$ $m_{0}^{2}$

$$
D\left(s, t, m^{2}\right)=A\left(s, t, m^{2}\right) e^{-i \delta} \sin \delta
$$

where $\delta$ is the elastic $\Lambda N$ phase shift. To see where this relation comes from we refer to Fig. 7: the appearance of the various unitarity cuts due to the baryon- 
baryon final state interaction leads to a discontinuity in the amplitude along the corresponding branch cut. We thus may read almost directly off the figure that, indeed, the discontinuity in the amplitude is given by the production amplitude times the two-baryon phase space density times the (complex conjugate of the) on-shell $\Lambda N T$-matrix ${ }^{10}$. Eq. (12) follows by simply using the definition of the elastic phase shift: $\kappa T\left(m^{2}\right)=e^{i \delta} \sin \delta$, where $\kappa=p^{\prime} \pi \mu$ denotes the phase space density here expressed in terms of the reduced mass of the $\Lambda N$ system $\mu=\left(M_{N} M_{\Lambda}\right) /\left(M_{N}+M_{\Lambda}\right)$.

We will discuss in sec. 7.4 how to control the possible influence of the $K$ interactions. The solution of (10) in the physical region can then be written as (see [38] and references therein)

$$
A\left(s, t, m^{2}\right)=e^{u\left(m^{2}+i 0\right)} \Phi\left(s, t, m^{2}\right)
$$

where

$$
\Phi\left(s, t, m^{2}\right)=\frac{1}{\pi} \int_{-\infty}^{\tilde{m}^{2}} \frac{d m^{\prime 2} D\left(s, t, m^{\prime 2}\right)}{m^{\prime 2}-m^{2}} e^{-u\left(m^{\prime 2}\right)}
$$

and, in the absence of bound states,

$$
u(z)=\frac{1}{\pi} \int_{m_{0}^{2}}^{\infty} \frac{\delta\left(m^{2}\right)}{m^{\prime 2}-z} d m^{\prime 2} .
$$

The $m^{2}$ dependence of $\Phi$ is dominated by the $m^{2}$ dependence of the production operator. The momentum transfer in the production operator, however, is controlled by the initial momentum and therefore one should expect the $m^{2}$ dependence of $\Phi$ to be weak as long as the corresponding relative momentum of the $\Lambda$-nucleon pair is small. Thus, in a large momentum transfer reaction, $\overline{10}$ The appearance of the complex conjugation here follows from the direct evaluation of the discontinuity of the $T$-matrix by employing the unitarity of the $S$ matrix: from $S^{\dagger} S=1$ it follows, that

$$
\operatorname{disc}(T):=\frac{1}{2 i}\left(T\left(m^{2}+i 0\right)-T\left(m^{2}-i 0\right)\right)=-\kappa\left|T\left(m^{2}\right)\right|^{2} .
$$


the $m^{2}$ dependence of the production amplitude $A$ is governed by the elastic scattering phase shifts of the dominant two particle reaction in the final state! The relation between the phase shifts and the $m^{2}$ dependence of the amplitude as can be extracted from an invariant mass distribution is fixed by analyticity and unitarity!

So far we are in line with the reasoning of the previous section, however the exponential factor in Eq. (13) is in general not simply the elastic scattering amplitude. For illustration we investigate the form of $A\left(m^{2}\right)$ for a final state interaction that is fully described by the first two terms in the effective range expansion (c.f. Eq. (7)), $p^{\prime} \operatorname{ctg}\left(\delta\left(m^{2}\right)\right)=1 / a+(1 / 2) r p^{\prime 2}$, where $p^{\prime}$ is the relative momentum of the final state particles under consideration in their center of mass system. Then $A$ can be given in closed form as [37]

$$
A\left(s, t, m^{2}\right)=\frac{\left(p^{2}+\alpha^{2}\right) r / 2}{1 / a+(r / 2) p^{\prime 2}-i p^{\prime}} \Phi\left(s, t, m^{2}\right)
$$

where $\alpha=(1 / r)(1+\sqrt{1+2 r / a})$. Note: The case of an attractive interaction without a bound state in this convention corresponds to a positive value of $a$ and a positive value of $r$. In the limit $a \rightarrow \infty$, as is almost realized in $N N$ scattering, the energy dependence of $A\left(m^{2}\right)$ is given by $1 /\left(1-i a p^{\prime}\right)$ as long as $p^{\prime} \ll 1 / r$. This, however, exactly agrees with the energy dependence for $N N$ on-shell scattering. This prediction was experimentally confirmed by the near-threshold measurements of the reaction $p p \rightarrow p p \pi^{0}[43,44]$. However, for interactions where the effective range is of the order of the scattering length, the numerator of Eq. (16) plays a role and thus the full production amplitude is no longer given by the on-shell elastic scattering times terms whose energy dependence is independent of the scattering parameters.

It is often argued that since the full production amplitude is given by a term with a plane wave final state as well as one with the strong interactions, that an appropriate parameterization is given by a Watson term (proportional to the elastic scattering of the outgoing particles) plus a constant term, and their relative strength should be taken as a free parameter. The considerations in the previous paragraphs show, however, that this is not the case: Eq. (13) (and thus also the special form given in Eq. (16)) describes the $m^{2}$ dependence of 

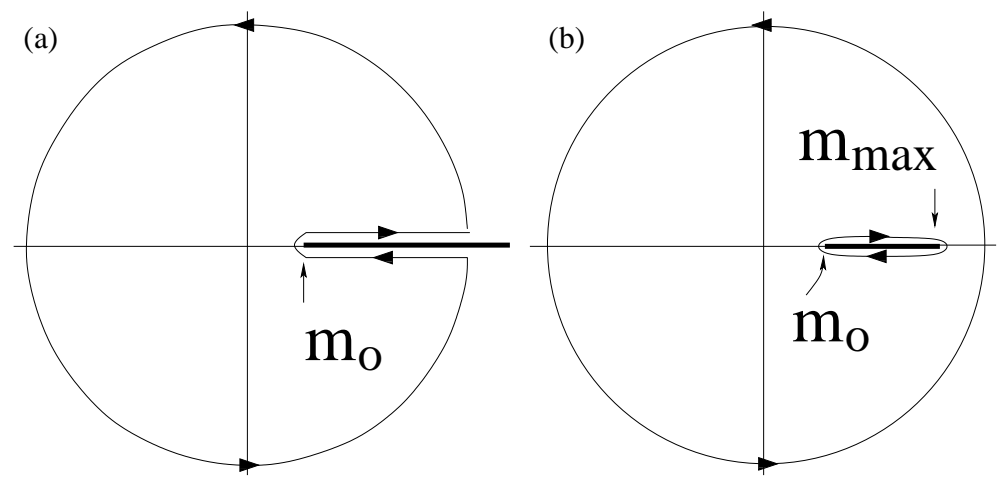

Fig. 8. The integration contours in the complex $m^{\prime 2}$ plane to be used in the original treatment by Geshkenbein [55,56] (a) and in the derivation of Eq. (18) (b). The thick lines indicate the branch cut singularities.

the full invariant mass spectrum.

One more comment is in order: In the previous section we stressed that, although the shape of the invariant mass spectra as well as the energy dependence of the total cross section are governed by the on-shell interactions in the subsystems, the overall normalization is not. This statement, based on effective field theory arguments, is illustrated in Appendix D. On the other hand, Eq. (13) gives a closed expression for the invariant mass spectrum for arbitrary values of $m^{2}$. On the first glance this look like a contradiction. However, it should be stressed that Eq. (13) does in general not allow one to relate the asymptotic form of the full production amplitude with information on the scattering parameters of the strongly interacting subsystems in the final state to the amplitude in the close to threshold regime. Besides the trivial observation that Eq. (13) holds for each amplitude individually and that far away from the threshold there should be many partial waves contributing, there is no reason to believe that the function $\Phi$ is constant over a wide energy range. Therefore, we want to emphasize that the $m^{2}$ dependence of Eq. (13) is controlled by the scattering phase shifts of the most strongly interacting subsystem only in a very limited range of invariant masses. In fact, the leading singularity that contributes to an $m^{2}$ dependence of $\Phi$ is that of the $t$-channel meson exchange in the production operator. The $m^{2}$ dependence that originates from this singularity can be estimated through a Taylor expansion of the momentum transfer and thus turns out to be governed by $\left(p^{\prime} / p\right)^{2}$, where $p^{\prime}$ denotes the relative momentum of the final state particle pair of interest. For 
$p$ we may use that of the initial state particles at the production threshold, all to be taken in the over all center of mass system. As a consequence, for values of $m^{2}$ that do not significantly deviate from $m_{0}^{2}$ (or equivalently $p^{\prime} \ll p$ ) the assumption of $\Phi$ being constant is justified. However $\Phi$ can show a significant $m^{2}$ dependence over a large range of invariant masses.

Let us now return to our main goal: namely to derive from (13) a formula that allows extraction of the elastic scattering phase shifts from an invariant mass spectrum. In this course we will derive a dispersion relation in terms of the function $\left|A\left(m^{2}\right)\right|^{2}$. Here we will use a method similar to the one used in $[55,56]$. However, in contrast to the formulas derived in these references, we will present a integral representation for the elastic scattering phase shifts from production data that involves a finite integration range only.

For this we first observe that Eq. (15) holds for purely elastic scattering only and thus is of very limited practical use. On the other hand a significant contribution stems from large values of $m^{\prime 2}$ which depends only weakly on $m^{2}$ in the near-threshold region, and therefore can be absorbed into the function $\Phi$. Let

$$
A\left(m^{2}\right)=\exp \left[\frac{1}{\pi} \int_{m_{0}^{2}}^{m_{\max }^{2}} \frac{\delta\left(m^{\prime 2}\right)}{m^{\prime 2}-m^{2}-i 0} d m^{\prime 2}\right] \tilde{\Phi}\left(m_{\text {max }}^{2}, m^{2}\right)
$$

where $\tilde{\Phi}\left(m_{\max }^{2}, m^{2}\right)=\Phi\left(m^{2}\right) \Phi_{m_{\max }^{2}}\left(m^{2}\right)$, with

$$
\Phi_{m_{\text {max }}^{2}}\left(m^{2}\right)=\exp \left[\frac{1}{\pi} \int_{m_{\text {max }}^{2}}^{\infty} \frac{\delta\left(m^{\prime 2}\right)}{m^{\prime 2}-m^{2}-i 0} d m^{\prime 2}\right]
$$

The quantity $m_{\max }^{2}$ is to be chosen by physical arguments in such a way that both $\Phi\left(m^{2}\right)$, and $\Phi_{m_{\text {max }}^{2}}\left(m^{2}\right)$ vary slowly on the interval $\left(m_{0}^{2}, m_{\max }^{2}\right)$. Obviously, it needs to be sufficiently large that the structure in the amplitude we are interested in can be resolved ${ }^{11}$. On the other hand, it should be as small as possible, since this will keep the influence of the inelastic channels

\footnotetext{
${ }^{11}$ One immediately observes that the integral given in Eq. (22) goes to zero for $m_{\max } \rightarrow m_{0}$. At the same time the theoretical uncertainty $\delta a^{m_{\max }}$, defined in Eq. (24), tends to the value of the scattering length.
} 
small. In order to estimate the minimal value of $m_{\max }$ we see from Eq. (16) that the values of $p^{\prime}$ that enter in the integral given in Eq. (22) should be at least large enough that the scattering length term plays a significant role. Thus we require $p_{\max }^{\prime} \sim 1 / a_{\text {typ }}$. In case of the $p n$ interaction (the spin triplet $p n$ scattering length is $5.4 \mathrm{fm}$ ) this would correspond to a value of $p_{\text {max }}^{\prime}$ of only $10 \mathrm{MeV}$ corresponding to a value for $\epsilon_{\max }=m_{\max }-m_{0}$ - the maximum excess energy that should occur within the integral in Eq. (17) (as well as Eq. (22) below) - as small as $2 \mathrm{MeV}$. On the other hand, if we want to study the hyperon-nucleon interaction, we want to 'measure' scattering lengths of the order of a few fermi we choose $a_{t y p}=1 \mathrm{fm}$, leading to a value of $40 \mathrm{MeV}$ for $\epsilon_{\max }$. Note that $40 \mathrm{MeV}$ is still significantly smaller than the thresholds for the closest inelastic channels (that for the $K \Sigma N$ final state is at $75 \mathrm{MeV}$, that for the $\pi \Lambda K N$ channel at $140 \mathrm{MeV})$.

The integral in (17) contains an unphysical singularity of the type $\log \left(m_{\max }^{2}-m^{2}\right)$, which is canceled by the one in $\tilde{\Phi}\left(m_{\max }^{2}, m^{2}\right)$, but this does not affect the region near threshold.

Notice next that the function

$$
\begin{aligned}
\frac{\log \left\{A\left(m^{2}\right) / \tilde{\Phi}\left(m_{\max }^{2}, m^{2}\right)\right\}}{\sqrt{\left(m^{2}-m_{0}^{2}\right)\left(m_{\text {max }}^{2}-m^{2}\right)}}= & \frac{1}{\sqrt{\left(m^{2}-m_{0}^{2}\right)\left(m_{\max }^{2}-m^{2}\right)}} \\
& \times \frac{1}{\pi} \int_{m_{0}^{2}}^{m_{\max }^{2}} \frac{\delta\left(m^{\prime 2}\right)}{m^{\prime 2}-m^{2}-i 0} d m^{\prime 2}
\end{aligned}
$$

has no singularities in the complex plane except the cut from $m_{0}^{2}$ to $m_{\max }^{2}$ (c.f. Fig. 8b) and its value below the cut equals the negative of the complex conjugate from above the cut. Hence,

$$
\begin{aligned}
& \frac{\delta\left(m^{2}\right)}{\sqrt{m^{2}-m_{0}^{2}}}= \\
& \quad-\frac{1}{2 \pi} \mathbf{P} \int_{m_{0}^{2}}^{m_{\max }^{2}} \frac{\log \left|A\left(m^{\prime 2}\right) / \tilde{\Phi}\left(m_{\max }^{2}, m^{\prime 2}\right)\right|^{2}}{\sqrt{m^{\prime 2}-m_{0}^{2}}\left(m^{\prime 2}-m^{2}\right)} \sqrt{\frac{m_{\max }^{2}-m^{2}}{m_{\max }^{2}-m^{\prime 2}}} d m^{\prime 2} .
\end{aligned}
$$

It is an important point to stress that 


$$
\mathbf{P} \int_{m_{0}^{2}}^{m_{\max }^{2}} \frac{1}{\sqrt{m^{\prime 2}-m_{0}^{2}}\left(m^{\prime 2}-m^{2}\right)} \sqrt{\frac{m_{\max }^{2}-m^{2}}{m_{\max }^{2}-m^{\prime 2}}} d m^{\prime 2}=0
$$

as long as $m^{2}$ is in the interval between $m_{0}^{2}$ and $m_{\text {max }}^{2}$. Therefore, if the function $\Phi$ only weakly depends on $m^{2}$, as it should in large momentum transfer reactions, it can well be dropped in the above equation. In addition, up to kinematical factors, $|A|^{2}$ agrees with the cross section for the production of a $\Lambda p$ pair of invariant mass $m$. We can therefore replace it in Eq. (20) by the cross section where, because of Eq. (21), all constant prefactors can be dropped. Thus we get

$$
\begin{array}{r}
a_{S}=\lim _{m^{2} \rightarrow m_{0}^{2}} \frac{1}{2 \pi}\left(\frac{M_{\Lambda}+M_{p}}{\sqrt{M_{\Lambda} M_{p}}}\right) \mathbf{P} \int_{m_{0}^{2}}^{m_{\max }^{2}} d m^{\prime 2} \sqrt{\frac{m_{\max }^{2}-m^{2}}{m_{\max }^{2}-m^{\prime 2}}} \\
\times \frac{1}{\sqrt{m^{\prime 2}-m_{0}^{2}}\left(m^{\prime 2}-m^{2}\right)} \log \left\{\frac{1}{p^{\prime}}\left(\frac{d^{2} \sigma_{S}}{d m^{\prime 2} d t}\right)\right\} .
\end{array}
$$

This is the desired formula: namely, the scattering length is expressed in terms of an observable. Note that Eq. (22) is applicable only if it is just a single $Y N$ partial wave that contributes to the cross section $\sigma_{S}$. In sec. 4.3 .1 we will discuss how the use of polarization in the initial state can be used to project out a particular spin state in the final state.

Up to the neglect of the kaon-baryon interactions, Eq. (20) is exact. Thus, $\delta a^{(t h)}$ — the theoretical uncertainty of the scattering length extracted using Eq. (22) - is given by the integral

$$
\begin{aligned}
\delta a^{(t h)}=- & \lim _{m^{2} \rightarrow m_{0}^{2}} \frac{1}{2 \pi}\left(\frac{M_{\Lambda}+M_{p}}{\sqrt{M_{\Lambda} M_{p}}}\right) \\
& \times \mathbf{P} \int_{m_{0}^{2}}^{m_{\max }^{2}} \frac{\log \left|\tilde{\Phi}\left(m_{\max }^{2}, m^{\prime 2}\right)\right|^{2}}{\sqrt{m^{\prime 2}-m_{0}^{2}}\left(m^{\prime 2}-m^{2}\right)} \sqrt{\frac{m_{\text {max }}^{2}-m^{2}}{m_{\text {max }}^{2}-m^{\prime 2}}} d m^{\prime 2} .
\end{aligned}
$$

Since $\log \left|\tilde{\Phi}\left(m_{\text {max }}^{2}, m^{2}\right)\right|^{2}=\log \left|\Phi\left(m^{2}\right)\right|^{2}+\log \left|\Phi_{m_{\text {max }}^{2}}\left(m^{2}\right)\right|^{2}$ we may write $\delta a^{(t h)}=\delta a^{(l h c)}+\delta a^{m_{\max }}$, where the former, determined by $\Phi\left(m^{2}\right)$, is controlled by the left hand cuts, and the latter, determined by $\Phi_{m_{\max }^{2}}\left(m^{2}\right)$, by the large-energy behavior of the $\Lambda N$ scattering phase shifts. The closest left 
hand singularity is that introduced by the production operator, which is governed by the momentum transfer. Up to an irrelevant overall constant, we may therefore estimate the variation of $\Phi \sim 1+\delta\left(p^{\prime} / p\right)^{2}$, where we assume $\delta$ to be of the order of 1 . Evaluation of the integral (23) then gives

$$
\delta a^{(l h c)} \sim \delta\left(p_{\max }^{\prime} / p^{2}\right) \sim 0.05 \mathrm{fm}
$$

where we used $p_{\max }^{\prime}{ }^{2}=2 \mu \epsilon_{\max }$ with $\epsilon_{\max } \sim 30 \mathrm{MeV}$ and the threshold value for $p \sim 900 \mathrm{MeV}$. On the other hand, using the definition of $\Phi_{m_{\max }^{2}}\left(m^{2}\right)$ given in Eq. (18) one easily derives

$$
\left|\delta a^{m_{\max }}\right|=\frac{2}{\pi p_{\max }^{\prime}}\left|\int_{0}^{\infty} \frac{\delta(y) d y}{\left(1+y^{2}\right)^{(3 / 2)}}\right| \leq \frac{2}{\pi p_{\max }^{\prime}}\left|\delta_{\max }\right|,
$$

where $y^{2}=\left(m-m_{\max }\right) / \epsilon_{\max }$. Thus, in order to estimate $\delta a^{m_{\max }}$ we need to make an assumption about the maximum value of the elastic $\Lambda N$ phase for $m^{2} \geq m_{\text {max }}^{2}$. Recall that we implicitly assume the inelastic channel not to play a role. This assumption was confirmed in Ref. [32] within a model calculation. The denominator in the integral appearing in Eq. (24) strongly suppresses large values of $y$. Since for none of the existing $\Lambda N$ models does $\delta_{\max }$ exceed $0.4 \mathrm{rad}$, we estimate

$$
\delta a^{m_{\max }} \sim 0.2 \mathrm{fm} .
$$

When using the phase shifts as given by the models directly in the integral, the value for $\delta a^{m_{\max }}$ is significantly smaller, since for all models the phase changes sign at energies above $m_{\max }^{2}$. Combining the two error estimates, we conclude

$$
\delta a \lesssim 0.3 \mathrm{fm}
$$

In the considerations of the theoretical uncertainty of the parameters extracted so far we did not talk about the possible effect of the kaon-baryon interaction. Unfortunately it can not be quantified a priori; however, it can be controlled experimentally. As will be discussed in sec. 4, a significant meson-baryon interaction, due, for example, to the presence of a resonance, will show up as a band in the Dalitz plot. If this band overlaps with the region of final state interactions we are interested in, interference effects might heavily distort the signal [57]. By choosing a different beam energy, the FSI region and the 
resonance band move away from each other. Thus there should be an energy regime where the FSI can be studied undistorted. It is therefore necessary for a controlled extraction of FSI parameters to do a Dalitz plot analysis at the same time. An additional possible cross-check of the influence of a mesonbaryon interaction would be to do the full analysis at two different beam energies separated in energy by at least the typical hadronic width (about 100-200 MeV). If the parameters extracted agree with each other, there was no substantial influence from other subsystems.

The possible gain in experimental accuracy for the extraction of the $\Lambda N$ scattering lengths is illustrated and discussed in detail in sec. 7.4.

To our knowledge so far the corresponding formalism including the Coulomb interaction is not yet derived.

\section{The initial state interaction}

As mentioned earlier, the collision energy of the two nucleons in the initial state is rather large, especially when we study the production of a heavy meson. However, as long as the excess energy is small, only very few partial waves contribute in the final state and thus the conservation of total angular momentum as well as parity and the Pauli principle allow for only a small number of partial waves in the initial state. In addition, only small total angular momenta are relevant. In such a situation the standard methods developed by Glauber, which include the initial state interaction via an exponential suppression factor [58], cannot be applied. The role of the initial state interaction relevant to meson production reactions close to the threshold was discussed for the first time in Ref. [40].

It should be clear a priori that for quantitative predictions the ISI has to play an important role: to allow a production reaction to proceed the nucleons in the initial state have to approach each other very closely-actually significantly closer that the range of the $N N$ interaction. Thus, especially for the production off heavy mesons, a large number of elastic and inelastic $N N$ reac- 
tions can happen before the two-nucleon pair comes close enough together to allow for the meson production. Thus the effective initial current gets reduced significantly.

Technically the inclusion of the initial state interaction in a calculation for a meson production process requires a convolution of the production operator with the half off-shell $T$-matrix for the scattering of the incoming particles (c.f. Fig. 5c) and d)). Contrary to the final state interaction, for the high initial energies the energy dependence of the $N N$ interaction is rather weak. One might therefore expect that the real part of the convolution integral is small and that the effect of the initial state interaction is dominated by the two nucleon unitarity cut [40]. This contribution, however, can be expressed completely in terms of on-shell $N N$ scattering parameters:

$$
\begin{aligned}
\left|\lambda_{L}\right|^{2} & =\left|\frac{1}{2} e^{i \delta_{L}(p)}\left(\eta_{L}(p) e^{i \delta_{L}(p)}+e^{-i \delta_{L}(p)}\right)\right|^{2} \\
& =-\eta_{L}(p) \sin ^{2}\left(\delta_{L}(p)\right)+\frac{1}{4}\left[1+\eta_{L}(p)\right]^{2} \leq \frac{1}{4}\left[1+\eta_{L}(p)\right]^{2},
\end{aligned}
$$

where $p$ denotes the relative momentum of the two nucleons in the initial state with the total energy $E$, and $\delta_{L}\left(\eta_{L}\right)$ denote the phase shift (inelasticity) in the relevant partial wave $L$. Each partial wave amplitude should be multiplied by $\lambda_{L}$, defined in Eq. (25), in order to account for the dominant piece of the ISI. This method was used e.g. in Ref. [59]. Using typical values for phase shifts and inelasticities at $N N$ energies that correspond to the thresholds of the production of heavier mesons, Eq. (25) leads to a reduction factor of the order of 3, thus clearly indicating that a consideration of the ISI is required for quantitative predictions. One should keep in mind, however, that Eq. (25) can only give a rough estimate of the effect of the initial state interaction and should whenever possible be replaced by a full calculation. This issue is discussed in detail in Ref. [34].

Unfortunately, the applicability of Eq. (25) is limited to energies where scattering parameters for the low $N N$ partial waves are available. Due to the intensive program of the EDDA collaboration for elastic proton-proton scattering [60] and the subsequent partial wave analysis documented in the SAID database [61], scattering parameters in the isospin-one channel are available 
up to energies that correspond to the $\phi$ production threshold. The situation is a lot worse in the isoscalar channel. Here $p n$ scattering data would be required. At present, those are available only up to the $\eta$ production threshold. It is very fortunate that there is a proposal in preparation to measure spin observables in the $p n$ system in this unexplored energy range at COSY [62].

\section{Observables}

In this section the various observables experimentally accessible are discussed. After some general remarks about the three body kinematics in the final state, in the next subsection we will discuss unpolarized observables. In the following subsection we will then focus on polarized observables.

Even if $x$ denotes just a single meson, the reaction $N N \rightarrow B_{1} B_{2} x$ is subject to a five dimensional phase: three particles in the final state introduce $3 \times$ $3=9$ degrees of freedom, but the four-momentum conservation reduces this number to 5 . As we will restrict ourselves to the near-threshold regime, the final state can be treated non-relativistically. The natural coordinate system is therefore given by the Jacobi-coordinates in the overall center of mass system (c.f. Ref. [42]), where first the relative momentum of one pair of particles is constructed and then the momentum of the third particle is calculated as the relative momentum of the third particle with respect to the two body system. Obviously there are three equivalent sets of variables possible. In the center of mass this choice reads

$$
\begin{aligned}
\vec{p}_{i j}{ }^{\prime} & =\frac{M_{i} \vec{p}_{j}-M_{j} \vec{p}_{i}}{M_{i}+M_{j}} \\
\vec{q}_{k} & =\frac{\left(M_{i}+M_{j}\right) \vec{p}_{k}-M_{k}\left(\vec{p}_{i}+\vec{p}_{j}\right)}{M_{i}+M_{j}+M_{k}}=\vec{p}_{k}
\end{aligned}
$$

where we labeled the three final state particles as $i j k ; q_{k}{ }^{\prime}=p_{k}$ holds in the over all center of mass system only. For simplicity in the following we will drop the subscripts when confusion is excluded. For reactions of the type $N N \rightarrow B_{1} B_{2} x$ it is common to work with the relative momentum of the twonucleon system and to treat the particle $x$ separately. From the theoretical 


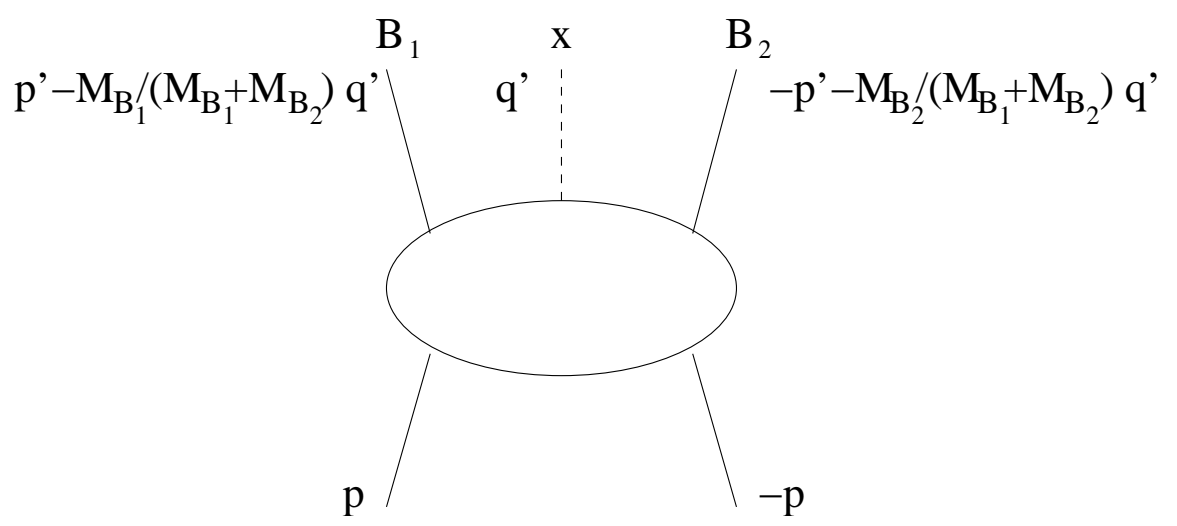

Fig. 9. Illustration of the choice of variables in the over all center-of-mass system.

point of view this choice is most convenient, for one is working already with the relative momentum of the dominant final state interaction and the assignment of partial waves as used in sec. 1.6 is straightforward. Note that for any given relative energy of the outgoing two nucleon system $\epsilon=p^{2} / M_{N}$ the modulus of the meson momentum $\left|\vec{q}^{\prime}\right|$ is fixed by energy conservation; we thus may characterize the phase space by the 5 tuple

$$
\xi=\left\{\epsilon, \Omega_{p^{\prime}}, \Omega_{q^{\prime}}\right\}
$$

where $\Omega_{k}=\left(\cos \left(\theta_{k}\right), \phi_{k}\right)$ denotes the angular part of vector $\vec{k}$. The coordinate system is illustrated in Fig. 9. The center of mass nucleon momentum in the initial state will be denoted by $\vec{p}$. Throughout this report we choose the beam axis along the $z$ axis. Explicit expressions for the vectors appearing are given in Appendix B.1.

Due to the high dimensionality of the phase space it is not possible to present the full complexity of the data in a single plot. At the end of sec. 4.2 we will discuss a possible way of presenting the data through integrations subject to particular constraints. A different choice would be simply to present highly differential observables as is done for bremsstrahlung ${ }^{12}$, or at least to use the two dimensional representation of the Dalitz plot to show some correlations.

\footnotetext{
${ }^{12}$ It should be noted that in the early bremsstrahlung experiments the method of integration was not possible, because the detectors used had very limited angular acceptance.
} 


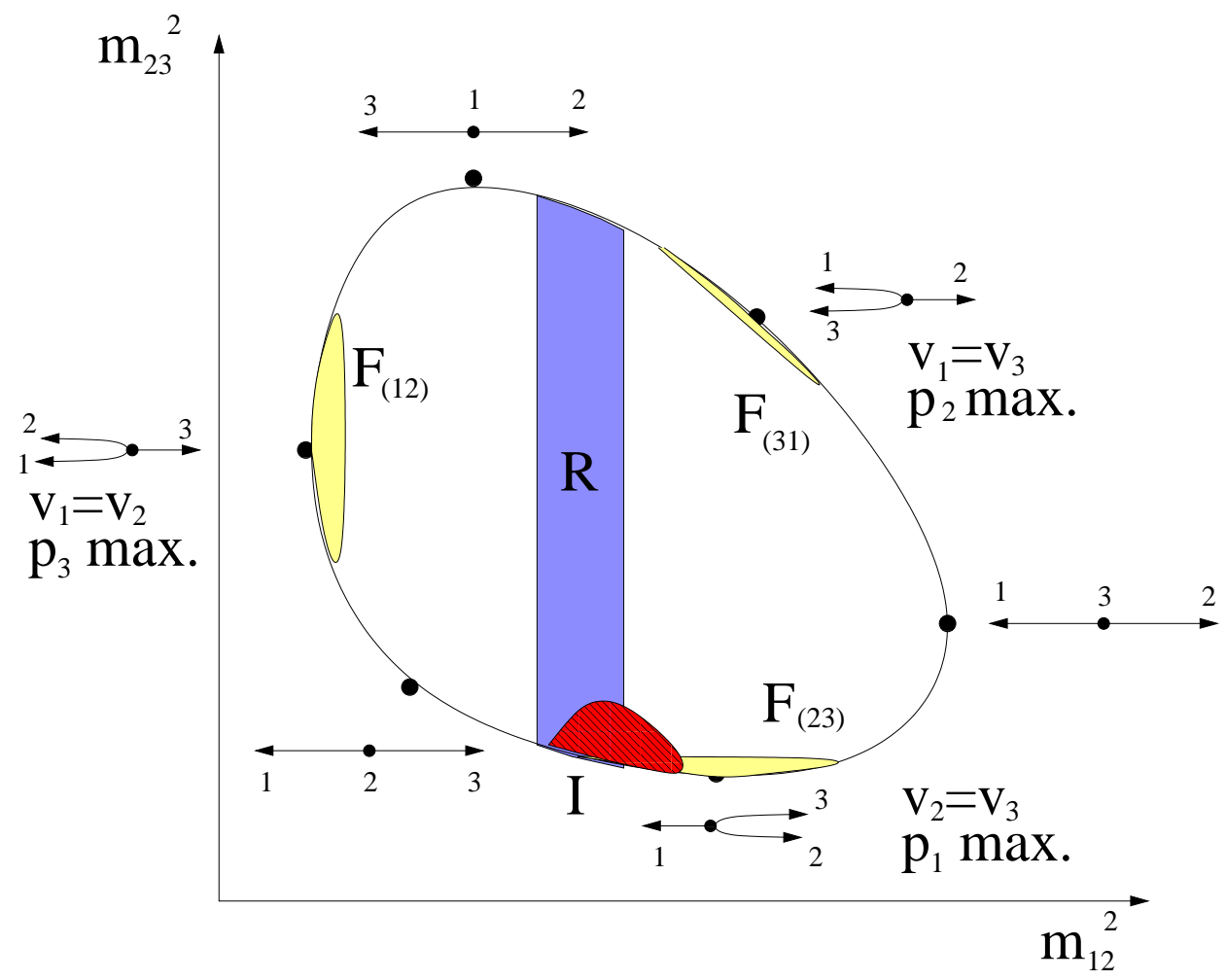

Fig. 10. Sketch of a Dalitz plot for a reaction with a three-body final state (particles are labeled as 1,2 and 3). Regions of possibly strong final state interactions in the (ij) system are labeled by $F_{(i j)}$; a resonance in the (12)-system would show up like the band labeled as $R$. The region of a possible interference of the two is labeled as I.

\subsection{Unpolarized observables}

As long as the initial state is unpolarized, the system has azimuthal rotation symmetry, reducing the number of degrees of freedom from 5 to 4 .

In the case of a three-particle decay (a $1 \rightarrow 3$ reaction) the physics does not depend on the initial direction. The same is true for reactions where the initial state does not define a direction, like in the experiment series for $\bar{p} p$ annihilation at rest carried out by the Crystal Barrel collaboration at LEAR (see Ref. [63] and references therein). Therefore the number of degrees of 
freedom is further reduced from four to two ${ }^{13}$. Those are best displayed in the so-called Dalitz plot (see [64] and references therein) that shows a two dimensional representation in the plane of the various invariant masses $m_{i k}^{2}=$ $\left(p_{i}+p_{k}\right)^{2}$. In reactions with two particles in the initial state and three particles in the final state $(2 \rightarrow 3$ reactions), however, the initial momentum defines an axis and therefore a single fully differential plot is no longer possible. Especially in the example given below it will become clear how the appearance of the additional momentum changes drastically the situation.

To account for the higher complexity of the $2 \rightarrow 3$ reactions, if enough statistics were collected in a particular experiment, one can present differential Dalitz plots - a new plot for each different orientation of the reaction plane [65]. However, in what follows we will call Dalitz plot the representation of a full data set/calculation in the plane of invariant masses, ignoring the initial direction. Obviously this means throwing out some correlations, for in general any integration reduces the amount of information in an observable. We will briefly review the properties of a Dalitz plot, closely following Ref. [64]. In Fig. 10 a schematic picture of it is shown. In this plot also different regimes are specified: those of potentially strong final state interaction for the subsystem $(i j)$ are labeled $F_{(i j)}$. They should occur when particle $i$ and $j$ move along very closely. On the other hand, resonances will show up as bands in the Dalitz plot. Labeled as $R$ in the figure, the effect of a resonance in the (12) system is shown. The total area of the Dalitz plot scales with the phase space volume. Therefore, as the excess energy decreases, resonance and final state interaction signals or the regions of different final state interactions might start to overlap, leading to interference phenomena (c.f. the hatched area $I$ in Fig. 10). It was demonstrated recently [57] that those can rather strongly distort resonance and final state interaction signals. At least for known final state properties, these patterns might help to better pin down resonance parameters [57]. On the other hand, as the excess energy increases the different structures move away from each other and one should then be able to study them individually.

\footnotetext{
$\overline{13}$ This is quite obvious, since one can look at a three particle decay as the crossed channel reaction of two body scattering which is well known to be characterized by two variables in the unpolarized case.
} 
This observation is of great relevance if one wants to extract parameters of a particular final state interaction from a production reaction (c.f. discussion in section 2).

In case of particle decays of spinless or unpolarized particles, the Dalitz plot not only contains the information about the occurrence of a resonance, but also its quantum numbers can be extracted by projecting the events in the resonance band (labeled as $R$ in Fig. 10) on the appropriate axis (for the example of the figure this is the 23 axis). In the case of $2 \rightarrow 3$ reactions, however, this projection is not necessarily conclusive. To explain this statement we have to have a closer look at the angles of the system. First of all there is a set of angles, the so called helicity angles, that can be constructed from the final momenta only. One example is

$$
\cos \left(\theta_{p^{\prime} q^{\prime}}\right)=\frac{\vec{p}^{\prime} \cdot \vec{q}^{\prime}}{\left|\vec{p}^{\prime}\right|\left|\vec{q}^{\prime}\right|},
$$

were $p^{\prime}$ and $q^{\prime}$ where defined in Eq. (26). These angles that can be extracted from the Dalitz plot directly. In addition there are those angles that are related to the initial momentum - the Jackson angles. One example is

$$
\cos \left(\theta_{p^{\prime} p}\right)=\frac{\vec{p}^{\prime} \cdot \vec{p}}{\left|\vec{p}^{\prime}\right||\vec{p}|}
$$

It should be stressed that it is not in the distribution of the helicity angles but that of the Jackson angles that the subsystems reflect their quantum numbers $[64]^{14}$. Therefore a pure Dalitz plot analysis is insufficient for production reactions and the distributions for the Jackson angles have to be studied as well. This will be illustrated in an example in the following subsection.

Note that in the presence of spin there are even additional axes in the problem. This will be discussed in detail in sec. 4.2.

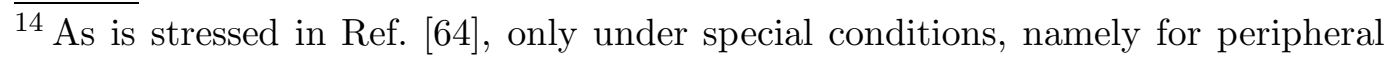
production as it occurs in high energy experiments, the information in the helicity angles and the Jackson angles agrees. Close to threshold, however, meson production is not at all peripheral.
} 

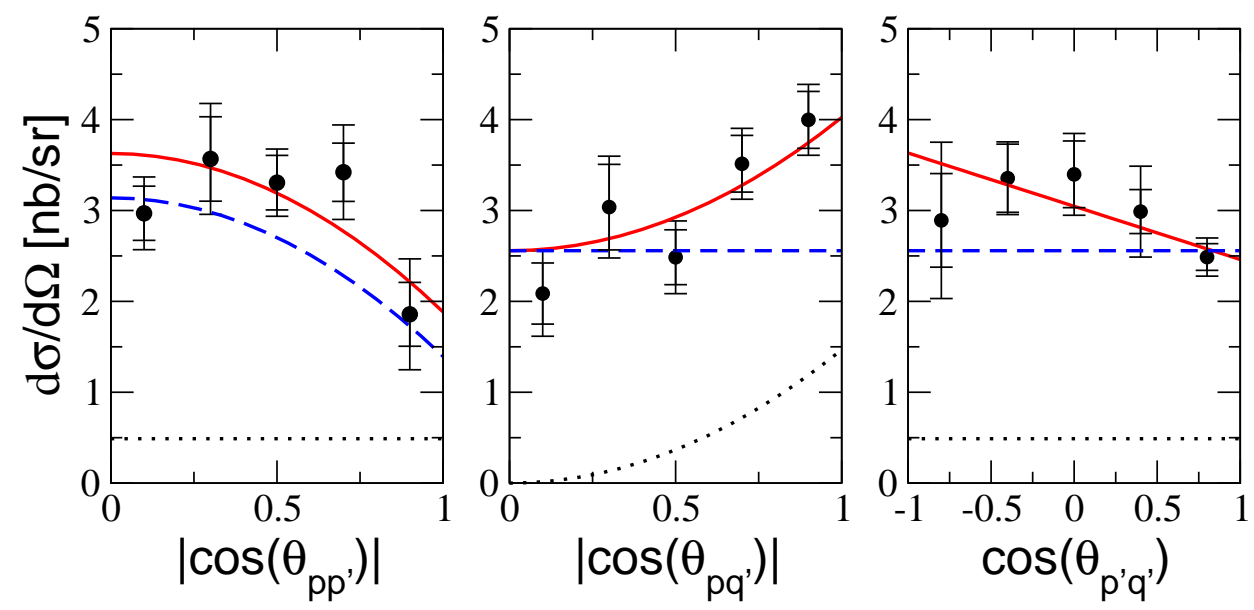

Fig. 11. Angular distributions for the reaction $p p \rightarrow d \bar{K}^{0} K^{+}$measured at $Q=46$ MeV [66]. The solid line shows the result of the overall fit including both $\bar{K} K$ $s$-waves as well as p-waves. To obtain the dashed (dotted) line, the parameters for the p-wave (s-wave) were set to zero (see text). The small error shows the statistical uncertainty only, whereas the large ones contain both the systematic as well as the statistical uncertainty added linearly.

\subsubsection{Example: analysis of $p p \rightarrow d \bar{K}^{0} K^{+}$close to threshold}

Recently, a first measurement of the reaction $p p \rightarrow d \bar{K}^{0} K^{+}$close to the production threshold was reported [66] at an excess energy $Q=46 \mathrm{MeV}$. The data, as well as the corresponding theoretical analysis, based on the assumption that only the lowest partial waves contribute, will now be used to illustrate the statements of the previous section. It will become clear, especially, that the information encoded in the distributions of the Jackson angles and the helicity angles is rather different.

As can be seen in table 2, a final state that contains $s$-waves only is not allowed in this reaction. For later convenience ${ }^{15}$ we work with the relative momentum of the kaon system $\left(\vec{p}_{\bar{K} K}{ }^{\prime} \equiv \vec{p}^{\prime}\right.$; c.f. Eqs. (26)) and the deuteron momentum with respect to this system $\left(\vec{q}_{d}{ }^{\prime} \equiv \vec{q}^{\prime}\right)$. Given our assumptions, that only the lowest partial waves contribute, the amplitudes that contribute to the production reaction are either linear in $q^{\prime}$ or linear in $p^{\prime}$. In Ref. [67]

\footnotetext{
$\overline{{ }^{15} \text { In sec. }} 7.5$ it will be argued, that the reaction $p p \rightarrow d \bar{K}^{0} K^{+}$can be used to study scalar resonance $a_{0}^{+}(980)$. Thus we are especially interested in the partial waves of the kaon system, that should show a strong final state interaction.
} 
Table 3

Results for the $C$ parameters from a fit to the experimental data. The parameters are given in units of $C_{0}^{q^{\prime}}$.

\begin{tabular}{ccccc}
\hline$C_{0}^{p^{\prime}}$ & $C_{0}^{q^{\prime}}$ & $C_{1}^{p^{\prime}}$ & $C_{1}^{q^{\prime}}$ & $C_{2}+\frac{1}{3} C_{3}$ \\
\hline $0 \pm 0.1$ & $1 \pm 0.03$ & $1.26 \pm 0.08$ & $-0.6 \pm 0.1$ & $-0.36 \pm 0.17$ \\
\hline
\end{tabular}

the full amplitude was constructed (c.f. also discussion in sec. 7.5), however it should be clear that the spin averaged square of the matrix element can be written as

$$
\begin{aligned}
|\overline{\mathcal{M}}|^{2}=C_{0}^{q^{\prime}} q^{\prime 2}+C_{0}^{p^{\prime}} p^{\prime 2}+C_{1}^{q^{\prime}}\left(\overrightarrow{q^{\prime}} \cdot \hat{p}\right)^{2}+C_{1}^{p^{\prime}}\left(\overrightarrow{p^{\prime}} \cdot \hat{p}\right)^{2} \\
+C_{2}\left(\overrightarrow{p^{\prime}} \cdot \overrightarrow{q^{\prime}}\right)+C_{3}\left(\overrightarrow{p^{\prime}} \cdot \hat{p}\right)\left(\overrightarrow{q^{\prime}} \cdot \hat{p}\right)
\end{aligned}
$$

since all terms in the amplitude are either linear in $\overrightarrow{p^{\prime}}$ or linear in $\overrightarrow{q^{\prime}}$. Here $\hat{p}=\vec{p} /|\vec{p}|$ denotes the beam direction. Since the two protons in the initial state are identical, any observable has to be symmetric under the transformation $\vec{p}=-\vec{p}$. This is why $\hat{p}$ appears in even powers only.

Figs. 11 and 12 show the data as well as a fit based on Eq. (30). The parameters extracted are given in table 3. The first two panels of Fig. 11 contain the distributions of the angles $\left(\overrightarrow{p^{\prime}} \cdot \hat{p}\right) / p^{\prime}$ and $\left(\overrightarrow{q^{\prime}} \cdot \hat{p}\right) / q^{\prime}$. The last panel contains the distribution of the helicity angle $\left(\overrightarrow{p^{\prime}} \cdot \overrightarrow{q^{\prime}}\right) /\left(q^{\prime} p^{\prime}\right)$. The solid line corresponds to a complete fit to the data including both $p$-waves in the $\bar{K}^{0} K^{+}$system as well as those in the $d\left(\bar{K}^{0} K^{+}\right)$system. For the long dashed line the $\bar{K}^{0} K^{+} p$-waves were set to zero, whereas for the dotted line the $d\left(\bar{K}^{0} K^{+}\right) p$-waves (corresponding to $\bar{K} K s$-waves) were set to zero. Thus, the first two panels truly reflect the partial wave content of the particular subsystems individually, whereas the helicity angle (which can also be extracted from the Dalitz plot) shows a flat distribution only if both subsystems are in a $p$-wave simultaneously. Therefore, the helicity angle can well be isotropic although one of the subsystems is in a high partial wave. Note that this statement is true even if all particles were spinless. The only thing that would change is that $C_{0}^{q^{\prime}}$ and $C_{0}^{p^{\prime}}$ would vanish (c.f. subsec. 4.3.2). In Fig. 12 two Dalitz plot projections (invariant mass spectra) are shown. The first one $\left(d \sigma / d m_{\bar{K} K}\right)$ is needed to disentangle $C_{0}^{q^{\prime}}$ and $C_{0}^{p^{\prime}}$. The second one does not give any additional information. 

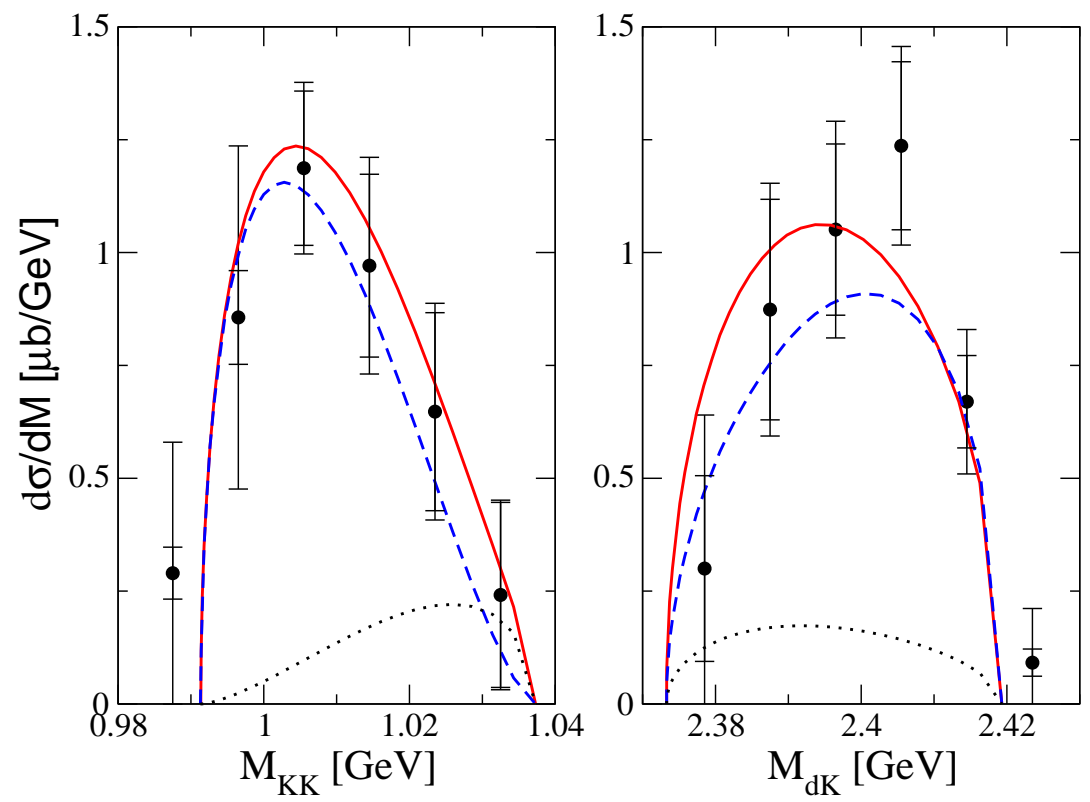

Fig. 12. Various mass distributions for the reaction $p p \rightarrow d \bar{K}^{0} K^{+}$(Line code as in Fig. 11). The small error bars show the statistical uncertainty only, whereas the large ones contain both the systematic as well as the statistical uncertainty (c.f. Ref. [66]).

What is now the information contained in the two-dimensional Dalitz plot? Since it does not contain any information about the initial state, the parts of the squared amplitude that can be extracted from the Dalitz plot are easily derived from Eq. (30) by integrating over the beam direction [64], giving

$$
\begin{aligned}
\int d \Omega_{p}|\overline{\mathcal{M}}|^{2}=\left(C_{0}^{q^{\prime}}+\frac{1}{3} C_{1}^{q^{\prime}}\right) q^{\prime 2}+( & \left.C_{0}^{p^{\prime}}+\frac{1}{3} C_{1}^{p^{\prime}}\right) p^{\prime 2} \\
& +\left(C_{3}+\frac{1}{3} C_{4}\right)\left(\overrightarrow{p^{\prime}} \cdot \overrightarrow{q^{\prime}}\right) .
\end{aligned}
$$

Therefore from the Dalitz plot one can extract the total $\bar{K} K s$-wave strength $\left(C_{0}^{q^{\prime}}+(1 / 3) C_{1}^{q^{\prime}}\right)$, the total $\bar{K} K p$-wave strength $\left(C_{0}^{p^{\prime}}+(1 / 3) C_{1}^{p^{\prime}}\right)$, as well as the strength of the interference of the two $\left(C_{3}+(1 / 3) C_{4}\right)$. Note that in this particular example, all the coefficients given in Eq. (31) (and even the $C_{0}$ and $C_{1}$ individually) can as well be extracted from the angular distributions given in Fig. 11 and the $\bar{K} K$ invariant mass distribution (left panel of Fig. 12) directly; the Dalitz plot here does not provide any additional information.

Obviously, as we move further away from the threshold, the complexity of the amplitude increases and the Dalitz plot contains information not revealed in 
the projections. To summarize, in order to allow for a complete analysis of the production data, in addition to the Dalitz plot the angular distributions of the final momenta on the beam momentum need to be analyzed as well. The latter distributions are the ones that give most direct access to the partial wave content of the subsystems.

\subsection{Spin dependent observables}

Polarization observables for $2 \rightarrow 2$ reactions are discussed in great detail in Ref. [68]. In our case, however, we have one more particle in the final state and therefore there are more degrees of freedom available. Here we will not only derive the expressions for the observables in terms of spherical tensors but also relate these to the partial wave amplitudes of the production matrix elements. In this section we closely follow Refs. [47,69].

In terms of the so called Cartesian polarization observables, the spindependent cross section can be written as

$$
\begin{aligned}
\sigma\left(\xi, \vec{P}_{b}, \vec{P}_{t}, \vec{P}_{f}\right)=\sigma_{0}(\xi)[1 & +\sum_{i}\left(\left(P_{b}\right)_{i} A_{i 0}(\xi)+\left(P_{f}\right)_{i} D_{0 i}(\xi)\right) \\
& +\sum_{i j}\left(\left(P_{b}\right)_{i}\left(P_{t}\right)_{j} A_{i j}(\xi)+\left(P_{b}\right)_{i}\left(P_{f}\right)_{j} D_{i j}(\xi)\right) \\
& \left.+\sum_{i j k}\left(P_{b}\right)_{i}\left(P_{t}\right)_{j}\left(P_{f}\right)_{k} A_{i j, k 0}(\xi) \ldots\right]
\end{aligned}
$$

where $\sigma_{0}(\xi)$ is the unpolarized differential cross section, the labels $i, j$ and $k$ can be either $x, y$ or $z$, and $P_{b}, P_{t}$ and $P_{f}$ denote the polarization vector of beam, target and the first one of the final state particles, respectively. All kinematic variables are collected in $\xi$, defined in Eq. (27). The observables shown explicitly in Eq. (32) include the beam analysing powers $A_{i 0}$, the corresponding quantities for the final state polarization $D_{0 i}$, the spin correlation coefficients $A_{i j}$, and the spin transfer coefficients $D_{i j}$. In this context it is important to note that baryons that decay weakly, as the hyperons do, have a self analyzing decay. In other words, the angular pattern of the decay particles depends on the polarization of the hyperon, therefore, the hyperon polariza- 
tion in the final state can be measured without an additional polarimeter (see e.g. Ref. [70]). All those observables that can be defined by just exchanging $\vec{P}_{b}$ and $\vec{P}_{t}$, such as the target analyzing power $A_{0 i}$, are not shown explicitly. From Eq. (32) it follows that for example

$$
\sigma_{0} A_{i j, k 0}=\frac{1}{\left(2 s_{b}+1\right)\left(2 s_{t}+1\right)} \operatorname{Tr}\left(\sigma_{k}^{(f)} \mathcal{M} \sigma_{i}^{(b)} \sigma_{j}^{(t)} \mathcal{M}^{\dagger}\right)
$$

where the $\sigma_{i}^{(b)}\left(\sigma_{j}^{(t)}\right)$ are the Pauli matrices acting in the spin space of beam and target, respectively. The production matrix element is denoted by $\mathcal{M}$. In addition, $s_{b}\left(s_{t}\right)$ denote the total spin of the beam (target) particles.

It is straightforward to relate the polarization observables to the partial wave amplitudes that can be easily extracted from any model. For this purpose it is convenient to use spherical tensors defined through

$$
\mathcal{T}_{k_{1} q_{1}, k_{2} q_{2}}^{k_{3} q_{3}, k_{4} q_{4}}=\frac{1}{\left(2 s_{b}+1\right)\left(2 s_{t}+1\right)} \operatorname{Tr}\left[\tau_{k_{3} q_{3}}^{\left(f_{1}\right) \dagger} \tau_{k_{4} q_{4}}^{\left(f_{2}\right) \dagger} \mathcal{M} \tau_{k_{1} q_{1}}^{(b)} \tau_{k_{2} q_{2}}^{(t)} \mathcal{M}^{\dagger}\right]
$$

where the $\tau_{k q}$ denote the spherical representation of the spin matrices

$$
\tau_{10}=\sigma_{z}, \tau_{1 \pm 1}=\mp \frac{1}{\sqrt{2}}\left(\sigma_{x} \pm i \sigma_{y}\right), \tau_{00}=1
$$

To relate the observables to the spherical tensors, the easiest method is to use the definitions of Eqs. (35) inside the various Eqs. (34). For the observables for which the final polarization remains undetected, the relations between the various $\mathcal{T}$ and the corresponding observables are shown in Table 4. In Table 5 a few of the observables that contain the final state polarization are listed. Triple polarization observables are not listed explicitly, but it is straightforward to derive also the relevant expressions for these, such as

$$
A_{x x, x}+A_{y y, x}-\left(A_{x y, y}-A_{y x, y}\right)=\sqrt{2}^{3} \operatorname{Re}\left(\mathcal{T}_{111-1}^{1100}\right)
$$

After some algebra given explicitly in Appendix C, one finds

$$
\mathcal{T}_{\rho}(\hat{p}, \hat{q})=\frac{1}{4} \sum_{\tilde{L} \tilde{l} \lambda} B_{\tilde{L} \tilde{l}, \lambda}^{Q}(\hat{q}, \hat{p}) \mathcal{A}_{\tilde{L} \tilde{l}, \lambda}^{\rho}
$$

where $\rho=\left\{k_{1} q_{1}, k_{2} q_{2}, k_{3} q_{3}, k_{4} q_{4}\right\}$ and $Q=q_{1}+q_{2}-q_{3}-q_{4}$. All the angular 

Cartesian Observable
$\mathcal{T}_{k_{1} q_{1} k_{2} q_{2}}$
$Q_{i}=q_{1}+q_{2}$

Differential cross section
$\sigma_{0}$
$\mathcal{T}_{0000}(s, \epsilon)$
0
*

Beam analyzing powers

$\begin{array}{cccc}\sigma_{0} A_{x 0} & -\sqrt{2} \operatorname{Re}\left(\mathcal{T}_{1100}(s, \epsilon)\right) & 1 & * \\ \sigma_{0} A_{y 0} & -\sqrt{2} \operatorname{Im}\left(\mathcal{T}_{1100}(s, \epsilon)\right) & 1 & \\ \sigma_{0} A_{z 0} & \mathcal{T}_{1000}(s, \epsilon) & 0 & *\end{array}$

Target analyzing powers

$\begin{array}{lcc}\sigma_{0} A_{0 x} & -\sqrt{2} \operatorname{Re}\left(\mathcal{T}_{0011}(s, \epsilon)\right) & 1 \\ \sigma_{0} A_{0 y} & -\sqrt{2} \operatorname{Im}\left(\mathcal{T}_{0011}(s, \epsilon)\right) & 1 \\ \sigma_{0} A_{0 z} & \mathcal{T}_{0010}(s, \epsilon) & 0\end{array}$
1

Spin correlation parameters

$\begin{array}{cccc}\sigma_{0} A_{z z} & \mathcal{T}_{1010}(s, \epsilon) & 0 & * \\ \sigma_{0} A_{\Sigma} & -2 \operatorname{Re}\left(\mathcal{T}_{111-1}(s, \epsilon)\right) & 0 & * \\ \sigma_{0} A_{\Delta} & 2 \operatorname{Re}\left(\mathcal{T}_{1111}(s, \epsilon)\right) & 2 & * \\ \sigma_{0} A_{x z} & -\sqrt{2} \operatorname{Re}\left(\mathcal{T}_{1110}(s, \epsilon)\right) & 1 & (*) \\ \sigma_{0} A_{z x} & -\sqrt{2} \operatorname{Re}\left(\mathcal{T}_{1011}(s, \epsilon)\right) & 1 & * \\ \sigma_{0} A_{y z} & -\sqrt{2} \operatorname{Im}\left(\mathcal{T}_{1110}(s, \epsilon)\right) & 1 & \\ \sigma_{0} A_{z y} & -\sqrt{2} \operatorname{Re}\left(\mathcal{T}_{1011}(s, \epsilon)\right) & 1 & \\ \sigma_{0}\left[A_{x y}+A_{y x}\right] & 2 \operatorname{Im}\left(\mathcal{T}_{1111}(s, \epsilon)\right) & 2 & * \\ \sigma_{0} A_{\Xi} & 2 \operatorname{Im}\left(\mathcal{T}_{111-1}(s, \epsilon)\right) & 0 & *\end{array}$

Table 4

Relations between spherical tensors and some observables that do not contain the final state polarization following Ref. [69]. To simplify notation, the indices specifying the final state polarization are dropped. The symbol * indicates a possible set of independent observables. Note: For pp induced reactions more observables become equivalent, as described in the text. Those are marked by $\left(^{*}\right)$. The linear combinations of spin correlation observables appearing in the table are defined in Eqs. (39). 


\begin{tabular}{|c|c|c|c|}
\hline Cartesian Observable & $\mathcal{T}_{k_{1} q_{1} k_{2} q_{2}}^{k_{3} q_{3} k_{4} q_{4}}$ & $Q=q_{1}+q_{2}-q_{3}-q_{4}$ & \\
\hline \multicolumn{4}{|l|}{ Induced polarization } \\
\hline$\sigma_{0} D_{0 x}$ & $\sqrt{2} \operatorname{Re}\left(\mathcal{T}_{0000}^{1-100}(s, \epsilon)\right)$ & 1 & $*$ \\
\hline$\sigma_{0} D_{0 y}$ & $\sqrt{2} \operatorname{Im}\left(\mathcal{T}_{0000}^{1-100}(s, \epsilon)\right)$ & 1 & \\
\hline$\sigma_{0} D_{0 z}$ & $\mathcal{T}_{0000}^{1000}(s, \epsilon)$ & 0 & $*$ \\
\hline \multicolumn{4}{|l|}{ Spin transfer coefficients } \\
\hline$\sigma_{0} D_{z z}$ & $\mathcal{T}_{1000}^{1000}(s, \epsilon)$ & 0 & $*$ \\
\hline$\sigma_{0} D_{\Sigma}$ & $2 \operatorname{Re}\left(\mathcal{T}_{1100}^{1100}(s, \epsilon)\right)$ & 0 & $*$ \\
\hline$\sigma_{0} D_{\Delta}$ & $-2 \operatorname{Re}\left(\mathcal{T}_{1100}^{1-100}(s, \epsilon)\right)$ & 2 & $*$ \\
\hline$\sigma_{0} D_{x z}$ & $-\sqrt{2} \operatorname{Re}\left(\mathcal{T}_{1100}^{1000}(s, \epsilon)\right)$ & 1 & $*$ \\
\hline$\sigma_{0} D_{z x}$ & $\sqrt{2} \operatorname{Re}\left(\mathcal{T}_{1000}^{1-100}(s, \epsilon)\right)$ & 1 & * \\
\hline$\sigma_{0} D_{y z}$ & $-\sqrt{2} \operatorname{Im}\left(\mathcal{T}_{1100}^{1000}(s, \epsilon)\right)$ & 1 & \\
\hline$\sigma_{0} D_{z y}$ & $\sqrt{2} \operatorname{Re}\left(\mathcal{T}_{1000}^{1-100}(s, \epsilon)\right)$ & 1 & \\
\hline$\sigma_{0}\left[D_{x y}+D_{y x}\right]$ & $-2 \operatorname{Im}\left(\mathcal{T}_{1100}^{1-100}(s, \epsilon)\right)$ & 2 & \\
\hline$\sigma_{0} D_{\Xi}$ & $2 \operatorname{Im}\left(\mathcal{T}_{1100}^{1100}(s, \epsilon)\right)$ & 0 & $*$ \\
\hline
\end{tabular}

Table 5

Relations between spherical tensors and some observables that do contain the final state polarization. The symbol * indicates a possible set of independent observables. The linear combinations of spin correlation observables appearing in the table are defined in Eqs. (40).

dependence is contained in

$$
B_{\tilde{L} \tilde{l}, \lambda}^{Q}(\hat{q}, \hat{p})=\sum_{\mu_{L}, \mu_{l}} \frac{1}{4 \pi}\left\langle\tilde{L} \mu_{L}, \tilde{l} \mu_{l} \mid \lambda Q\right\rangle Y_{\tilde{L} \mu_{L}}(\hat{p}) Y_{\tilde{l} \mu_{l}}(\hat{q})
$$

and

$$
\mathcal{A}_{\tilde{L} \tilde{l}, \lambda}^{\rho}=\sum_{\alpha, \bar{\alpha}} C_{\tilde{L} \tilde{l}, \lambda}^{\alpha, \bar{\alpha}, \rho} M^{\alpha}\left(M^{\bar{\alpha}}\right)^{\dagger} .
$$

Here $\alpha$ and $\bar{\alpha}$ are multi-indices for all the quantum numbers necessary to characterize a particular partial wave matrix element and $C$ denotes a coupling coefficient that can be expressed in terms of Clebsch-Gordan coefficients. Its 
explicit form is given in Eq. (C.9). From Eq. (36) one can derive the angular dependences of all observables.

In what follows it is convenient to define the following quantities:

$$
A_{\Sigma}=A_{x x}+A_{y y}, A_{\Delta}=A_{x x}-A_{y y}, \text { and } A_{\Xi}=A_{x y}-A_{y x}
$$

and, analogously,

$$
D_{\Sigma}=D_{x x}+D_{y y}, D_{\Delta}=D_{x x}-D_{y y}, \text { and } D_{\Xi}=D_{x y}-D_{y x}
$$

Using the conservation of parity and the explicit expression for the $C$ coefficient given in Eq. (C.9)

$$
C_{\tilde{L} \tilde{l}, \lambda}^{\alpha, \bar{\alpha}, \rho}=(-)^{\left(k_{1}+k_{2}+k_{3}+k_{4}\right)} C_{\tilde{L} \tilde{l}, \lambda}^{\bar{\alpha}, \alpha, \rho}
$$

Since the parameter $C$ is real, the analyzing powers are proportional to the imaginary part of $M_{\alpha} M_{\bar{\alpha}}^{*}$, whereas the differential cross section as well as the spin correlation parameters depend on the real part of $\mathcal{M}_{\alpha} \mathcal{M}_{\bar{\alpha}}^{*}$. Thus, it is either the real part or the imaginary part of $B$ that contributes to the angular structure. As we will see in the following subsection, this observation allows for a straight-forward identification of the possible azimuthal dependences of each observable. Another obvious consequence of Eq. (41) is, that those observables for which $\sum k_{i}$ is odd have to be small when only a single partial wave dominates. Thus, at the threshold, analyzing powers will vanish.

The structure of Eq. (36) is general - no assumption regarding the number of contributing partial waves was necessary. However, if we want to make statements about the expected angular dependence of observables, the number of partial waves needs to be restricted. For example, if we allow for at most $p$-waves for the $N N$ system as well as the particle $x$ with respect to the $N N$ system, then the largest value of $\tilde{L}$ and $\tilde{l}$ that can occur is 2 , which strongly limits the possible $\theta$-dependences that can occur in the angular function $B$ defined in Eq. (37).

If the spin of the particles is not detected there is no interference between different spin states in the final state. This leads to a severe selection rule 
in case of the reaction $p p \rightarrow p p X$ : since the final state is necessarily in a $T=1$ state the Pauli principle demands that different spin be accompanied by different angular momentum. Therefore, for the a reaction with a $p p$ final state the partial waves can be grouped into two sets, namely $\{S s, S d, D s, D p\}$ and $\{P p, P s\}$, where only the members of the individual sets interfere with each other.

\subsubsection{Equivalent observables}

All the angular dependence of the observables is contained in the function $B$ defined in Eq. (37). In this subsection we will discuss some properties of $B$ and relate these to properties of particular observables.

The functional form of $B$ enables one immediately to read off the allowed azimuthal dependences for each observable as well as to identify equivalent observables. To see this we rewrite Eq. (37) as

$$
B_{\tilde{L} \tilde{l}, \lambda}^{Q}(\hat{q}, \hat{p})=\sum_{n} f_{\tilde{L} \tilde{l}, \lambda, Q, n}\left(\theta_{p^{\prime}}, \theta_{q^{\prime}}\right) \exp \left\{i\left[(Q-n) \phi_{p^{\prime}}+n \phi_{q^{\prime}}\right]\right\}
$$

which directly translates into the following $\phi$-dependences for the spherical tensors (c.f. Eq. (36)):

$$
\mathcal{T}_{\rho}(\hat{p}, \hat{q})=\sum_{n=-N}^{N} g_{\rho, n}\left(\theta_{p^{\prime}}, \theta_{q^{\prime}}\right) \exp \left\{i\left[(Q-n) \phi_{p^{\prime}}+n \phi_{q^{\prime}}\right]\right\}
$$

Note that $N$ is given by the highest partial waves that contribute to the reaction considered:

$$
-\tilde{L}_{\max } \leq(Q-N) \leq \tilde{L}_{\max } \text { and } N \leq \tilde{l}_{\max }
$$

where $\tilde{L}_{\max }\left(\tilde{l}_{\max }\right)$ is given by twice the maximum baryon-baryon (meson) angular momentum. These limits are inferred by the Clebsch-Gordan coefficient appearing in the definition of $B$ in Eq. (37).

Eq. (43) directly relates the real and the imaginary parts of the spherical tensors:

$$
\operatorname{Im}\left(\mathcal{T}_{\rho}\left(\theta_{p^{\prime}}, \phi_{p^{\prime}}+\pi /(2 Q), \theta_{q^{\prime}}, \phi_{q^{\prime}}+\pi /(2 Q)\right)\right)=\operatorname{Re}\left(\mathcal{T}_{\rho}\left(\theta_{p^{\prime}}, \phi_{p^{\prime}}, \theta_{q^{\prime}}, \phi_{q^{\prime}}\right)\right)
$$


Thus, two observables are equivalent if they are given by the real and imaginary parts of the same spherical tensor with $Q \neq 0$. In table 4 the relations of the various observables to the spherical tensors are given. Thus, using Eq. (45) we can identify the following set of pairwise equivalent observables:

$$
A_{y 0} \equiv A_{x 0}, A_{0 y} \equiv A_{0 x}, A_{x x}-A_{y y} \equiv A_{x y}+A_{y x}
$$

and analogously for observables for which the final state polarization is measured as well. Notice that there is no connection between $A_{x x}+A_{y y}$ and $A_{x y}-A_{y x}$, for these have $Q=0$ and therefore there is no transformation, such as the one given in Eq. (45), that relates real and imaginary parts of the spherical tensors.

For identical particles in the initial state, as in $p p$ induced reactions, all observables should be equivalent under the exchange of beam and target. This further reduces the number of independent observables, for now the beam analyzing powers are equivalent to the target analyzing powers and $A_{x z}$ is equivalent to $A_{z x}$. In tables 4 and 5 a possible set of independent observables is marked by a *. Those of these that are not independent for identical particles in the initial state are labeled as $(*)$.

From the discussion in the previous section it follows (c.f. Eq. (41)), that all those observables with an even (odd) value of $k_{1}+k_{2}+k_{3}+k_{4}$ lead to a real (imaginary) value for $g_{\rho, n}$, defined in Eq. (43). As a consequence, for all coefficients appearing in the expansion of the observables, the $\phi$-dependence is fixed (c.f. table (4)); for example, the terms that contribute to $\sigma_{0}, \sigma_{0} A_{z z}$, and $\sigma_{0} A_{\Sigma}$ behave as $\cos \left(n\left(\phi_{q^{\prime}}-\phi_{p^{\prime}}\right)\right)$.

As was stressed above, the phase space for the production reactions is of high dimension. To allow for a proper presentation of the data as well as of calculations, one either needs high dimensional plots (see discussion in the previous section) or the dimensionality needs to be reduced to one dimensional quantities ${ }^{16}$, while, however, still preserving the full complexity of the data. As

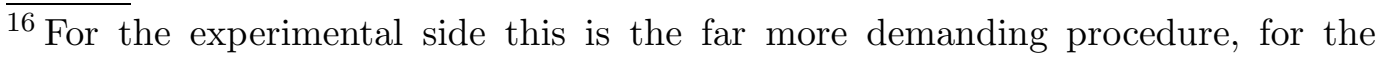
angular dependence of efficiency as well as acceptance needs to be known very well over the full angular range for those variables that are integrated in order not
} 

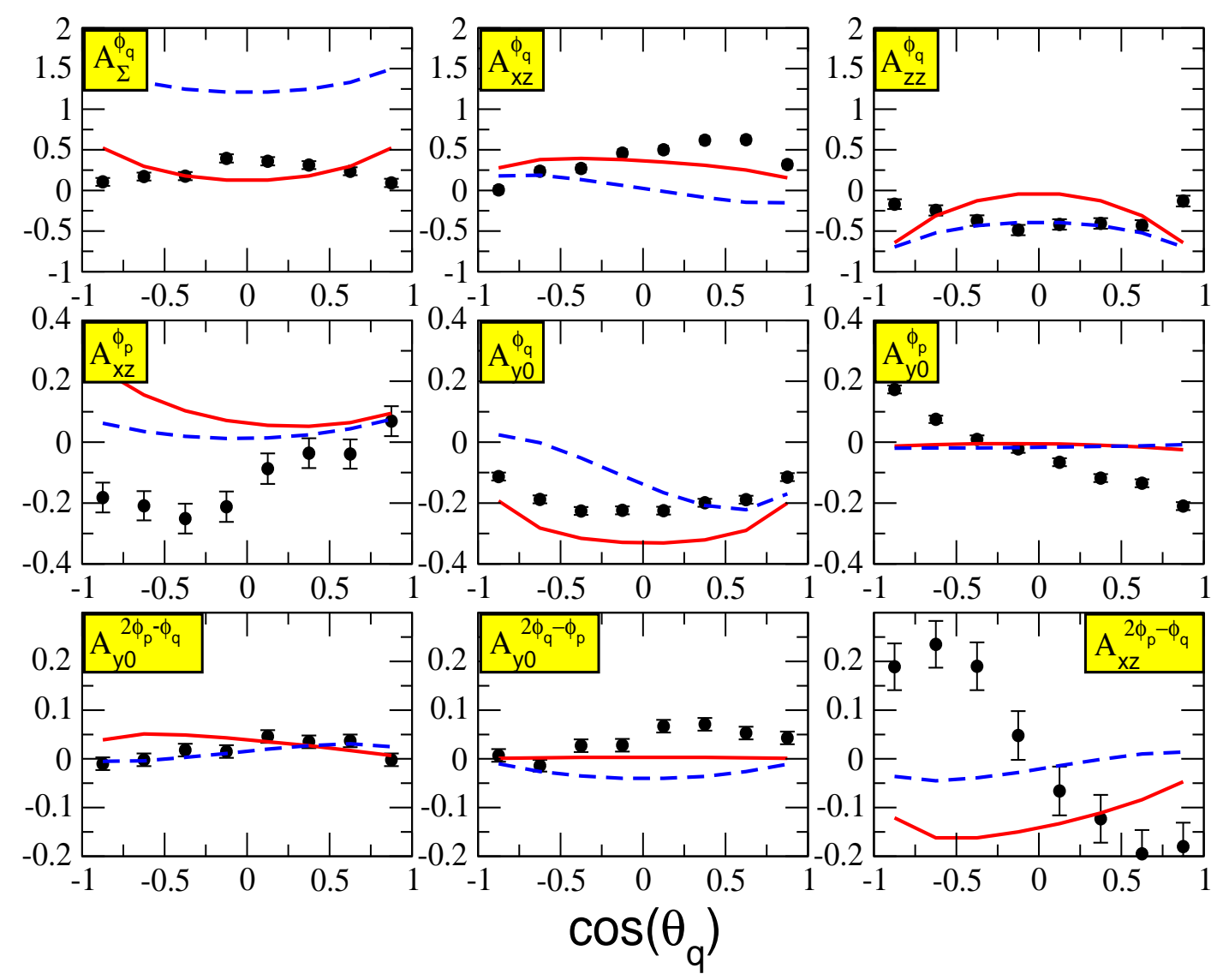

Fig. 13. Some polarization observables reported in Ref. [47] for the reaction $\vec{p} \vec{p} \rightarrow p p \pi^{0}$ at $\eta=0.83$ as a function of the pion angle compared to predictions of the model of Ref. [71]. The solid lines show the results for the full model whereas contributions from the Delta where omitted for the dashed lines.

can be seen in Eq. (43), each polarization observable is described by $2 N+1$ functions $g_{\rho, n}\left(\theta_{p^{\prime}}, \theta_{q^{\prime}}\right)$, where the number of relevant terms is given by the number of partial waves. In order to allow disentanglement of these functions, in Ref. [47] it was proposed to integrate each observable over both azimuthal angles under a particular constraint,

$$
\Phi=m \phi_{p}+n \phi_{q}=c
$$

This integration projects on those terms that depend on $\Phi$ or do not show any azimuthal dependence at all [47]. To further reduce the dimensionality of the data, either the relative proton angle or the meson angle can be integrated to leave one with a large number of observables that depend on one parameter only. Those are then labeled as $A_{i j}^{\Phi}\left(\theta_{k}\right)$, where $k$ is either $p$ or $q$. In Figs. 13

to introduce false interferences. 

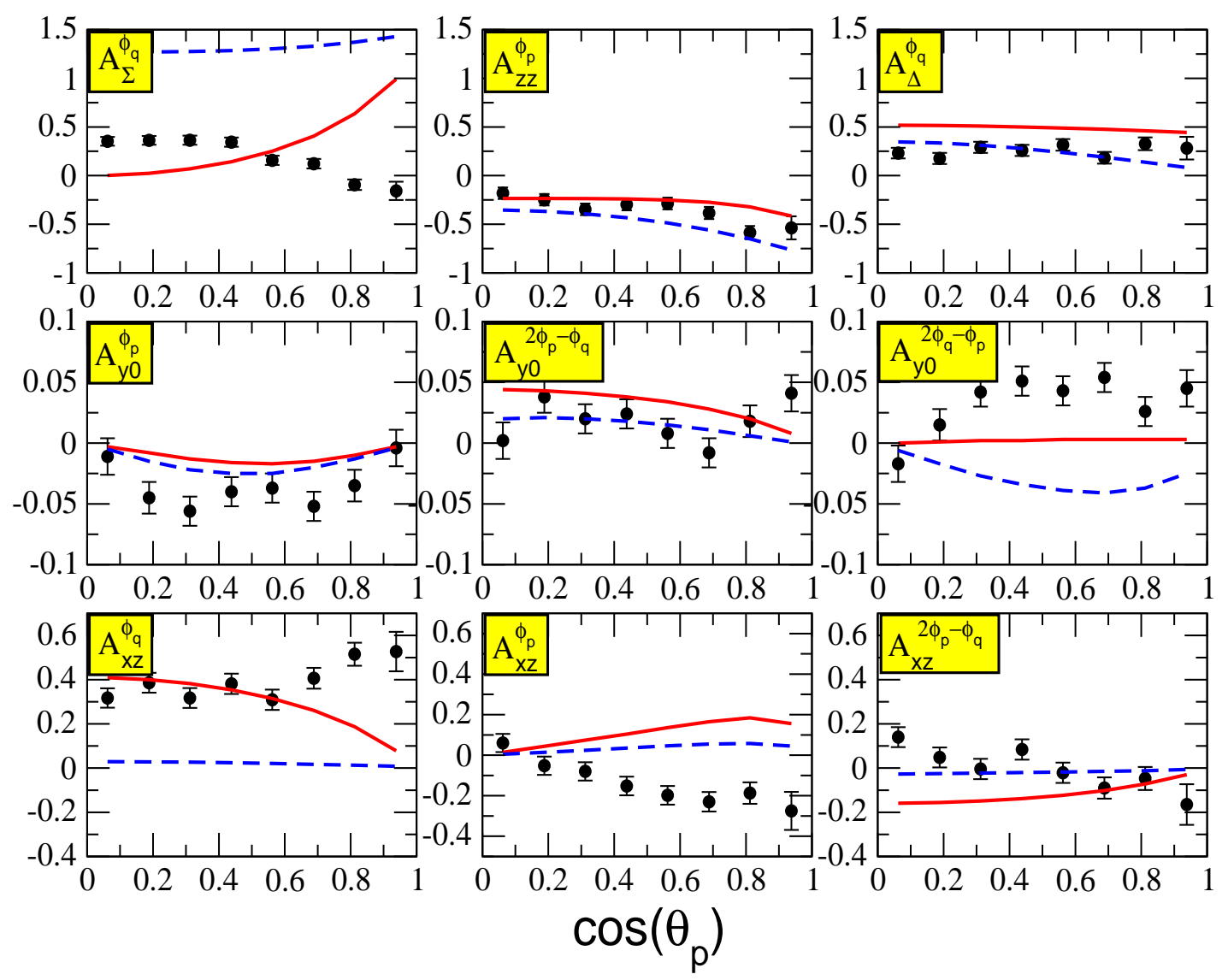

Fig. 14. Some polarization observables reported in Ref. [47] for the reaction $\vec{p} \vec{p} \rightarrow p p \pi^{0}$ at $\eta=0.83$ as a function of $\cos \left(\theta_{p}\right)$ compared to predictions of the model of Ref. [71]. The solid lines show the results for the full model whereas contributions from the Delta were omitted for the dashed lines.

and 14 some observables reported in Ref. [47] are shown for the energy with highest statistics, namely $\eta=0.83$. In the figures the data is compared to the model predictions of Ref. [71]. The solid lines are the results of the full model whereas for the dashed lines the contribution from the Delta isobar was switched off. In section 6 this model will be discussed in more detail.

In the case of Ref. [47] the complete set of polarization observables for the reaction $\vec{p} \vec{p} \rightarrow p p \pi^{0}$ is given. Since the particles in the initial state are identical there are 7 independent observables, all functions of 5 independent parameters (c.f. table 4). In the analysis of the data presented in the same reference it was assumed that only partial waves up to $p$-waves in both the $N N$ as well as the $(N N) \pi$ system were relevant. Thus the various integrations described in the previous paragraph lead to 32 independent integrated observables that depend only on a single parameter. On the other hand, if the assumption 
about the maximum angular momenta holds, only 12 partial waves have to be considered in the partial wave analysis. Since the amplitudes are complex and two phases are not observable (c.f. discussion at the end of section 4.2), a total of 22 degrees of freedom needs to be fixed from the data. Thus a complete partial wave decomposition for the reaction $\overrightarrow{p p} \rightarrow p p \pi^{0}$ seems feasible.

\subsection{General structure of the amplitudes}

In this section we give the recipe for constructing the most general transition amplitude for reactions of the type $N N \rightarrow B_{1} B_{2} x$, where we focus on spin $1 / 2$ baryons in the final state. A generalization to other reactions is straightforward. For further applications we refer to a recent review [72].

For simplicity let us restrict ourselves to those reactions in which there is only one meson produced. The system is then characterized by three vectors,

$$
\vec{p}, \quad \vec{q}^{\prime}, \text { and } \vec{p}^{\prime}
$$

denoting the relative momentum of the two nucleons in the initial state, the meson momentum, and the relative momentum of the two nucleons in the final state, respectively - in the over all center of mass system. In addition, as long as $x$ denotes a scalar or pseudoscalar meson, we find 6 axial vectors, namely those that can be constructed from the above:

$$
i\left(\vec{p} \times \vec{p}^{\prime}\right), \quad i\left(\vec{p} \times \vec{q}^{\prime}\right), \quad \text { and } i\left(\vec{p}^{\prime} \times \vec{q}^{\prime}\right) ;
$$

and those that contain the final or initial spin of the two nucleon system

$$
\vec{S}, \quad \vec{S}^{\prime}, \text { and } i\left(\vec{S} \times \vec{S}^{\prime}\right)
$$

where $\vec{S}=\chi_{1}^{T} \sigma_{y} \vec{\sigma} \chi_{2}$ and $\vec{S}^{\prime}=\chi_{3}^{\dagger} \vec{\sigma} \sigma_{y}\left(\chi_{4}^{\dagger}\right)^{T}$. Here $\chi_{i}$ denotes the Pauli spinors for the incoming $(1,2)$ and outgoing $(3,4)$ nucleons and $\vec{\sigma}$ denotes the usual Pauli spin matrices. If $x$ is a vector particle, an additional axial vector, namely the polarization vector of the vector meson $\vec{\epsilon}^{*}$ occurs. In addition, if instead of a two nucleon state in the continuum a deuteron occurs in the final state, its polarization direction will be characterized by the same $\vec{\epsilon}^{*}$. Since the energy 
available for the final state is small (we focus on the close to threshold regime), we restrict ourselves to a non relativistic treatment of the outgoing particles. This largely simplifies the formalism since a common quantization axis can be used for the whole system.

In order to construct the most general transition amplitude that satisfies parity conservation, we have to combine the vectors and axial vectors given above so that the final expression form a scalar or pseudoscalar for reactions where the produced meson has positive or negative intrinsic parity, respectively. The most general form of the transition matrix element may be written as

$$
\mathcal{M}=H\left(\mathcal{I} \mathcal{I}^{\prime}\right)+i \vec{Q} \cdot\left(\vec{S} \mathcal{I}^{\prime}\right)+i \vec{A} \cdot\left(\vec{S}^{\prime} \mathcal{I}\right)+\left(S_{i} S_{j}{ }^{\prime}\right) B_{i j}
$$

where $\mathcal{I}=\left(\chi_{2}^{T} \sigma_{y} \chi_{1}\right)$ and $\mathcal{I}^{\prime}=\left(\chi_{4}^{\dagger} \sigma_{y}\left(\chi_{3}^{T}\right)^{\dagger}\right)$. In addition, the amplitudes have to satisfy the Pauli Principle as well as invariance under time reversal. This imposes constraints on the terms that are allowed to appear in the various coefficients.

The 9 amplitudes that contribute to $B$ may be further decomposed according to the total spin to which $\vec{S}$ and $\vec{S}^{\prime}$ may be coupled

$$
B_{i j}=b^{s} \delta_{i j}+b_{k}^{v} \epsilon_{i j k}+b_{i j}^{t}
$$

where the superscripts indicate if the combined spin of the initial and the final state are coupled to $0(s), 1(v)$ or $2(t)$, where $b_{i j}^{t}$ is to be a symmetric, trace free tensor.

Once the amplitudes are identified the evaluation of the various observables is straightforward. In this case the polarization comes in through

$$
\chi_{i} \chi_{i}^{\dagger}=\frac{1}{2}\left(1+\vec{P}_{i} \cdot \vec{\sigma}\right)
$$

where $\vec{P}_{i}$ denotes the polarization direction of particle $i$. Using the formulas given in Appendix B.2 one easily derives (summation over equal indices is implied): 


$$
\begin{gathered}
4 \sigma_{0}=|H|^{2}+|\vec{Q}|^{2}+|\vec{A}|^{2}+\left|B_{m n}\right|^{2} \\
4 A_{0 i} \sigma_{0}=+i \epsilon_{i j k}\left(Q_{j}^{*} Q_{k}+B_{j l}^{*} B_{k l}\right)+2 \operatorname{Im}\left(B_{i l}^{*} A_{l}-Q_{i}^{*} H\right) \\
4 D_{0 i} \sigma_{0}=-i \epsilon_{i j k}\left(A_{j}^{*} A_{k}+B_{l j}^{*} B_{l k}\right)-2 \operatorname{Im}\left(B_{l i}^{*} Q_{l}-A_{i}^{*} H\right) \\
4 A_{i j} \sigma_{0}=\delta_{i j}\left(-|H|^{2}+|Q|^{2}-|A|^{2}+\left|B_{m n}\right|^{2}\right) \\
+2 \operatorname{Re}\left(\epsilon_{l i j}\left(Q_{l}^{*} H-A_{m}^{*} B_{l m}\right)-Q_{i}^{*} Q_{j}-B_{i m}^{*} B_{j m}\right) \\
4 D_{i j} \sigma_{0}=2 \operatorname{Re}\left(Q_{i}^{*} A_{j}+\epsilon_{i l m} Q_{l}^{*} B_{m j}\right. \\
\left.+\epsilon_{j m l} B_{i l} A_{m}^{*}+\frac{1}{2} \epsilon_{i l m} \epsilon_{j n k} B_{l n}^{*} B_{m k}+B_{i j}^{*} H\right) \\
4 A_{i j, k 0} \sigma_{0}=\operatorname{Im}\left(\delta_{i j}\left(2 H^{*} A_{k}-2 Q_{l}^{*} B_{l k}-\epsilon_{\alpha \beta k}\left(A_{\alpha}^{*} A_{\beta}-B_{l \alpha}^{*} B_{l \beta}\right)\right)\right. \\
+2 \epsilon_{l i j}\left(Q_{l}^{*} A_{k}-\epsilon_{n m k} A_{n}^{*} B_{l m}+H B_{l k}^{*}\right) \\
\left.+2\left(Q_{i}^{*} B_{j k}+Q_{j}^{*} B_{i k}\right)-\epsilon_{m n k}\left(B_{i m}^{*} B_{j n}+B_{j m}^{*} B_{i n}\right)\right)
\end{gathered}
$$

For illustration we also give here the explicit expressions for $A_{\Sigma}$ and $A_{\Delta}$ defined in the previous section:

$$
\begin{aligned}
\sigma_{0} A_{\Sigma} & =\frac{1}{2}\left(-|H|^{2}+\left|Q_{z}\right|^{2}-|\vec{A}|^{2}+\left|B_{z n}\right|^{2}\right) \\
\sigma_{0} A_{\Delta} & =-\frac{1}{2}\left(Q_{x}^{*} Q_{x}-Q_{y}^{*} Q_{y}+B_{x m}^{*} B_{x m}-B_{y m}^{*} B_{y m}\right) .
\end{aligned}
$$

As was stressed in the previous section, the method of spherical tensors is very well suited to identifying equivalent observables. This is significantly more difficult in the amplitude approach. However the amplitude method becomes extremely powerful if - due to physical arguments or appropriate kinematical cuts - one of the subsystems can be assumed in an $s$-wave, for then the number of available vectors is reduced significantly and rather general arguments become possible (c.f. sec. 4.3.1).

Since any amplitude can be made successively more complex by multiplying it by an arbitrary scalar, in most of the cases an ordering scheme is demanded in order to make the approach useful. In the near-threshold regime this is given by the power of final momenta occurring - in analogy with the partial wave expansion. Actually, the amplitude approach presented here and the partial wave expansion presented in the previous subsection are completely equivalent. However, in the near-threshold regime the amplitude method is 

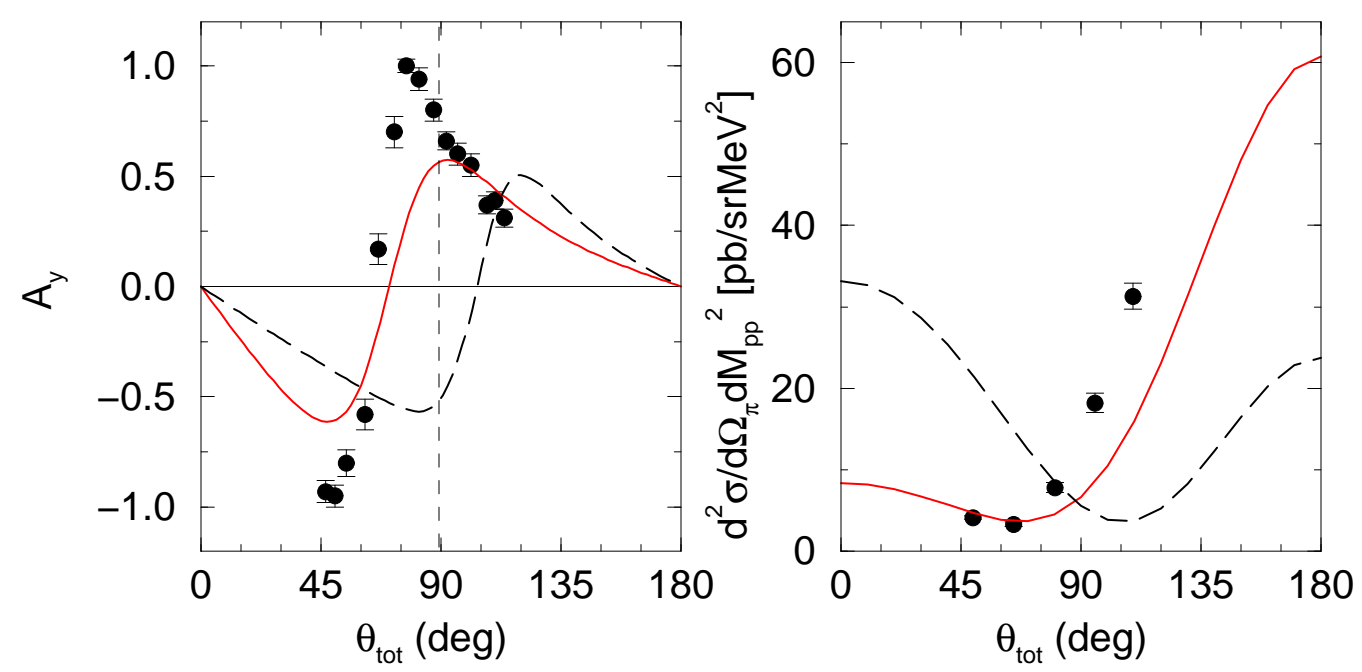

Fig. 15. Sensitivity of the analyzing power as well as the differential cross section for the reaction $p n \rightarrow p p \pi^{-}$to the sign of the ${ }^{3} P_{0} \rightarrow{ }^{1} S_{0} s$ amplitude $a_{1}$. The lines corresponds to the model of Ref. [73]: the solid line is the model prediction whereas for the dashed line the sign of $a_{1}$ was reversed. The experimental data are from Ref. [74] and [75] at $T_{L a b}=353 \mathrm{MeV}(\eta=0.65)$.

more transparent. As one goes away from the threshold the number of partial waves contributing as well as the number of the corresponding terms in the amplitude expansion increases rapidly. As a consequence the construction of the most general transition amplitude is rather involved. The partial wave expansion, on the other hand, can be easily extended to an arbitrary number of partial waves.

As follows directly from Eq. (48), in the general case the matrix element $\mathcal{M}$ is described by 16 complex valued scalar functions. One can show, e.g. by explicit construction, that for general kinematics of the reaction $N N \rightarrow N N x$ all 16 amplitudes are independent. A possible choice is given explicitly in Table 6 for the reaction $p p \rightarrow p p+$ (pseudoscalar). However, for particular reaction channels or an appropriate kinematical situation their number sometimes reduces drastically. For example in collinear kinematics (where $\vec{p}, \vec{q}^{\prime}$, and $\vec{p}^{\prime}$ are all parallel) the number of amplitudes that fully describes the reactions $p p \rightarrow p p+$ (pseudoscalar) is equal to 3 (this special case is discussed in Ref. [76]). This can be directly read off Table 6, for under collinear conditions all cross products vanish and all structures of one group 
Table 6

List of a possible set of the independent amplitude structures that contribute to the reaction $p p \rightarrow p p+$ (pseudoscalar). In the last column shows the lowest partial waves for the final state that contribute to the given amplitude (using the notation of section 1.6). To keep the expressions simple we omitted to give the symmetric, trace free expressions for the terms listed in the last two lines.

\begin{tabular}{|l|lll|l|}
\hline Amplitudes & \multicolumn{2}{|l|}{ Structures } & & Lowest pw \\
\hline \hline$H$ & $\left(\vec{p} \times \vec{p}^{\prime}\right) \vec{q}^{\prime}\left(\vec{p} \cdot \vec{p}^{\prime}\right)$ & & Ds \\
\hline$\vec{Q}$ & $\vec{p}$ & $\vec{p}^{\prime}\left(\vec{p} \cdot \vec{p}^{\prime}\right)$ & $\vec{q}^{\prime}\left(\vec{p} \cdot \vec{q}^{\prime}\right)$ & Ss, Ds, Sd \\
\hline$\vec{A}$ & $\vec{p}\left(\vec{p} \cdot \vec{p}^{\prime}\right)$ & $\vec{q}^{\prime}\left(\vec{p}^{\prime} \cdot \vec{q}^{\prime}\right)$ & Ps, Pd \\
\hline$B_{i j}$ & $\vec{p}^{\prime}$ & $\epsilon_{i j k} p_{k}^{\prime}\left(\vec{p} \cdot \vec{q}^{\prime}\right)$ & $\epsilon_{i j k} q_{k}^{\prime}\left(\vec{p}^{\prime} \cdot \vec{p}\right)$ & Pp \\
& $\epsilon_{i j k} p_{k}\left(\vec{p}^{\prime} \cdot \vec{q}^{\prime}\right)$ & $\left(\vec{p} \times \vec{q}^{\prime}\right)_{i} p_{j}^{\prime}$ & \\
& $\delta_{i j}\left(\vec{p} \times \vec{p}^{\prime}\right) \vec{q}^{\prime}$ & $\left(\vec{p} \times \vec{p}^{\prime}\right)_{i} q_{j}^{\prime}$ & $\vec{p}^{\prime}$ & \\
& $p_{i} p_{j}\left(\vec{p} \times \vec{p}^{\prime}\right) \vec{q}^{\prime}$ & $\left(\vec{p} \times \vec{p}^{\prime}\right)_{i} p_{j}\left(\vec{p} \cdot \vec{q}^{\prime}\right)$ & $\left(\vec{p} \times \vec{q}^{\prime}\right)_{i} p_{j}\left(\vec{p} \cdot \vec{p}^{\prime}\right)$ & \\
\hline
\end{tabular}

that are given by the different vectors of the system collapse to one structure $\left(\vec{Q} \rightarrow \alpha \vec{p}, \quad \vec{A} \rightarrow \beta p, \quad B_{i j} \rightarrow \gamma \epsilon_{i j k} p_{k}\right)$.

Another interesting example is that of elastic $p p$ scattering. The Pauli Principle demands (c.f. section 1.6) that odd (even) parity states are in a spin triplet (singlet). Time reversal invariance requires the amplitude to be invariant under the interchange of the final and the initial state. When parity conservation is considered in addition, one also finds that the total spin is conserved. Thus, only $H$ and $B_{i j}$ will contribute to elastic $p p$ scattering. In addition, from the two vectors available in the system $\left(\vec{p}\right.$ and $\left.\vec{p}^{\prime}\right)$ one can construct only 4 structures that contribute to the latter, namely $\delta_{i j}\left(\vec{p} \cdot \vec{p}^{\prime}\right) b_{1}, \epsilon_{i j k}\left(\vec{p} \times \vec{p}^{\prime}\right)_{k} b_{2}$, $\left(p_{i} p_{j}^{\prime}+p_{j} p_{i}^{\prime}-(2 / 3) \delta_{i j}\right)\left(\vec{p} \cdot \vec{p}^{\prime}\right) b_{3}$, and $\left(p_{i} p_{j}+p_{j}^{\prime} p_{i}^{\prime}-(2 / 3) \vec{p}^{2} \delta_{i j}\right)\left(\vec{p} \cdot \vec{p}^{\prime}\right) b_{4} \cdot{ }^{17}$ Thus, $p p$ scattering is completely characterized by 5 scalar functions.

As a further example and to illustrate how the formalism simplifies in the vicinity of the production threshold, we will now discuss in detail the production of pions in $N N$ collisions. Throughout this report, however, the formalism will be $\overline{{ }^{17} \text { Note }}$, this choice of structures in not unique; we could also have used $\left(\vec{p} \times \vec{p}^{\prime}\right)_{i}(\vec{p} \times$ $\left.\vec{p}^{\prime}\right)_{j}$ as a replacement of any other structure in $b_{i j}^{t}$. 
applied to various reactions. In our example there are three reaction channels experimentally accessible, namely $p p \rightarrow p p \pi^{0}, p p \rightarrow p n \pi^{+}$, and $p n \rightarrow p p \pi^{-}$, that can be expressed in terms of the three independent transition amplitudes $\mathcal{A}_{T_{i} T_{f}}$ (as long as we assume isospin to be conserved), where $T_{i}\left(T_{f}\right)$ denote the total isospin of the initial (final) $N N$ system [27] (c.f. section 1.5). As in section 1.6, we will restrict ourselves to those final states that contain at most one $p$-wave. We may then write for the amplitudes of $\mathcal{A}_{11}$,

$$
\begin{aligned}
H^{11} & =0, \\
\vec{Q}^{11} & =a_{1} \hat{p}, \\
\vec{A}^{11} & =a_{2} \vec{p}^{\prime}+a_{3}\left[\left(\hat{p} \cdot \vec{p}^{\prime}\right) \hat{p}-\frac{1}{3} \vec{p}^{\prime}\right], \\
B_{i j}^{11} & =0 ;
\end{aligned}
$$

for the amplitudes of $\mathcal{A}_{10}$,

$$
\begin{aligned}
H^{10} & =0 \\
\vec{Q}^{10} & =0 \\
\vec{A}^{10} & =b_{2} \vec{q}^{\prime}+b_{3}\left[\left(\vec{q}^{\prime} \cdot \hat{p}\right) \hat{p}-\frac{1}{3} \vec{q}^{\prime}\right], \\
\vec{B}_{i j}^{10} & =\epsilon_{i j k} b_{1} \hat{p}_{k} ;
\end{aligned}
$$

for the amplitudes of $\mathcal{A}_{01}$,

$$
\begin{aligned}
H^{01} & =0, \\
\vec{Q}^{01} & =c_{1} \vec{q}^{\prime}+c_{2}\left[\hat{p}\left(\hat{p} \cdot \vec{q}^{\prime}\right)-\frac{1}{3} \vec{q}^{\prime}\right], \\
\vec{A}^{01} & =0, \\
B_{i j}^{01} & =\epsilon_{n m k} \vec{p}_{k}{ }^{\prime}\left(c_{3} \delta_{i n} \delta_{j m}+c_{4} \delta_{j m}\left(\hat{p}_{i} \hat{p}_{n}-\frac{1}{3} \delta_{i n}\right)\right. \\
& \left.+c_{5} \delta_{i n}\left(\hat{p}_{j} \hat{p}_{m}-\frac{1}{3} \delta_{j m}\right)\right),
\end{aligned}
$$

where $\hat{p}$ denotes the initial $N N$ momentum, normalized to 1 , and $\vec{p}^{\prime}$ and $\vec{q}^{\prime}$ denote the final nucleon and pion relative momentum, respectively. The coefficients given are directly proportional to the corresponding partial wave amplitudes as listed in Table 1; e.g., $a_{3}$ is proportional to the transition amplitude ${ }^{1} D_{2} \rightarrow{ }^{3} P_{2} s$. When constructing amplitude structures for higher partial waves care has to be taken not to list dependent structures. In order to remove 
dependent structures the reduction formula Eq. (B.7) proved useful. In addition, one should take care that the number of coefficients appearing exactly matches the number of partial waves allowed. For example, the partial waves that contribute to $B_{i j}^{01}$ are ${ }^{3} S_{1} \rightarrow{ }^{3} P_{1} s,{ }^{3} D_{1} \rightarrow{ }^{3} P_{1} s$ and ${ }^{3} D_{2} \rightarrow{ }^{3} P_{2} s$.

The large number of zeros appearing in the above list of amplitude structures reflects the strong selection rules discussed in section 1.6.

As an example we will calculate the beam analyzing power and the differential cross section for the reaction $\overrightarrow{p n} \rightarrow p p \pi^{-}$. These observables were measured at TRIUMF $[74,75]$ and later at PSI $[77,78]$ - here, however, with a polarized neutron beam). In accordance with the TRIUMF experiment, where the relative $N N$ energy in the final state was restricted to at most $7 \mathrm{MeV}$, we assume that the outgoing $N N$ system is in a relative $S$-wave. This largely simplifies the expressions. We then find

$$
\begin{aligned}
& \sigma_{0}= \frac{1}{4}\left|a_{1}\right|^{2}+\frac{1}{2} q^{\prime} \operatorname{Re}\left(a_{1}^{*}\left(c_{1}+\frac{2}{3} c_{2}\right)\right) \cos (\theta), \\
& \sigma_{0} A_{y}=\left(\frac{1}{4} q^{\prime 2} \operatorname{Im}\left(c_{1}^{*} c_{2}\right) \sin (2 \theta)\right. \\
&\left.\quad-\frac{1}{2} q^{\prime} \operatorname{Im}\left(a_{1}^{*}\left(c_{1}-\frac{1}{3} c_{2}\right)\right) \sin (\theta)\right) \cos (\phi),
\end{aligned}
$$

where we used the definitions $\left(\vec{q}^{\prime} \times \hat{p}\right)_{y}=-q^{\prime} \sin (\theta) \cos (\phi)$ and $\left(\vec{q}^{\prime} \cdot \hat{p}\right)=$ $q^{\prime} \cos (\theta)$ (c.f. Appendix B.1). Thus, the forward-backward asymmetry in $\sigma_{0}$ as well as the shift of the zero in $A_{y}$ directly measure the relative phase of the ${ }^{3} P_{0} \rightarrow{ }^{1} S_{0} s$ transition in $A_{11}\left(a_{1}\right)$ in the pion $p$-wave transitions of $A_{01}$ $\left(c_{1}\right.$ and $\left.\left.c_{2}\right)\right)$, as was first pointed out in Ref. [79]. This issue will be discussed below (c.f. sec. 6). Note: As before we neglected here pion $d$-waves, since they are kinematically suppressed close to the threshold (c.f. Eq. (9)).

\subsubsection{Example I: Polarization observables for a baryon pair in the ${ }^{1} S_{0}$ final state}

As an example of the efficiency of the amplitude method, in this subsection we will present an analysis of the angular pattern of some polarization observables for the reaction $p p \rightarrow B_{1} B_{2} x$ under the constraint that the outgoing two 
baryon state $\left(B_{1} B_{2}\right)$ is in the ${ }^{1} S_{0}$ partial wave and $x$ is a pseudoscalar, as is relevant for the reaction $p p \rightarrow p K \Lambda$. The dependence of the observables on the meson emission angle is largely constrained under these circumstances, as was stressed in Ref. [80]. In contrast to the discussion in sec. 1.6, here we will not assume $B_{1}$ and $B_{2}$ to be identical particles. The results of this subsection will show how to disentangle in a model independent way the two spin components of the hyperon-nucleon interaction [32].

The analysis starts with identifying the tensors that are to be considered in the matrix element of Eq. (48) ${ }^{18}$. For this we go through a chain of arguments similar to those in sec. 1.6. Given that we restrict ourselves to a spin-zero final state, only $H$ and $\vec{Q}$ can be non-zero. In addition, the quantum numbers of the final state are fixed by $l_{x}$, the angular momentum of the pseudoscalar with respect to the two baryon system, since

$$
J=l_{x} \quad \text { and } \quad \pi_{t o t}=(-)^{\left(l_{x}+1\right)}
$$

for the total angular momentum and the parity, respectively. Conservation of parity and angular momentum therefore gives

$$
(-)^{L}=(-)^{l_{x}+1}=(-)^{J+1} \quad \longrightarrow \quad S=1
$$

and consequently we get $H=0$. In addition, for odd values of $l_{x}$ we see from the former equation that $L$ must be even. In $p p$ systems, however, even values of $L$ correspond to $S=0$ states, not allowed in our case. Therefore $l_{x}$ must be even. We may thus make the following ansatz:

$$
\vec{Q}=\alpha \vec{p}+\beta \vec{q}^{\prime}\left(\vec{q}^{\prime} \cdot \vec{p}\right)
$$

where $\alpha$ and $\beta$ are even functions of $p, p^{\prime}$ and $\left(\vec{p} \cdot \vec{p}^{\prime}\right)$. All other coefficients appearing in Eq. (48) vanish. This has serious consequences for the angular dependences of the various observables. For example, the expression for the analyzing power collapses to

\footnotetext{
$\overline{18 \text { Note, }}$ here we could as well refer to table 6 to come to the same conclusion as in this section, for the ${ }^{1} S_{0}$ state is allowed for the $p p$ system. However, the argument given is quite general and instructive.
} 


$$
A_{0 i} \sigma_{0}=\frac{i}{4} \epsilon_{i j k}\left(Q_{j}^{*} Q_{k}\right)=\frac{1}{2} \operatorname{Im}\left(\beta^{*} \alpha\right)\left(\vec{q}^{\prime} \cdot \vec{p}\right)\left(\vec{q}^{\prime} \times \vec{p}\right)_{i}
$$

Therefore, independently of the partial wave of the pseudoscalar emitted, for a two-baryon pair in the ${ }^{1} S_{0}$ state the analyzing power $A_{0 y}$ vanishes if the pseudoscalar is emitted either in the $x y$-plane or in the $z x$-plane. In Ref. [32] this observation was used to disentangle the different spin states of the $\Lambda N$ interaction.

\subsubsection{Example II: Amplitude analysis for $p p \rightarrow d \bar{K}^{0} K^{+}$close to threshold}

In sec. 4.1.1 we discussed in some detail the data of Ref. [66] for the reaction $p p \rightarrow d \bar{K}^{0} K^{+}$based on rather general arguments on the cross section level. In this subsection we will present the corresponding production amplitude based on the amplitude method presented above. This study will allow us at the same time to extract information on the relative importance of the $a_{0}^{+}$and the $\Lambda(1405)$ in the reaction dynamics.

As in sec. 4.1.1, we assume that either the $\bar{K} K$ system or the deuteron with respect to the $\bar{K} K$ system is in a $p$-wave, whereas the other system is in an $s$-wave, calling for an amplitude linear in $p^{\prime}$ or $q^{\prime}$, respectively. We use the same notation as in sec. 4.1.1. Therefore the final state has odd parity and thus also the amplitude needs to be odd in the initial momentum $\vec{p}$. An odd parity isovector $N N$ state has to be $S=1$ and thus has to be linear in $\vec{S}$, defined in section 4.3. In addition, the deuteron in the final state demands that each term is linear in the deuteron polarization vector $\epsilon$. We therefore get for the full transition amplitude, in slight variation to Eq. (48) due to the presence of the deuteron in the final state,

$$
\mathcal{M}=B_{i j} \vec{S}_{i} \vec{\epsilon}_{j}^{*}
$$

where $\vec{\epsilon}$ appears as complex conjugate, since the deuteron is in the final state, and

$$
\begin{aligned}
B_{i j}= & a_{S p} \hat{p}_{i}{\overrightarrow{q_{j}}}^{\prime}+b_{S p}\left(\hat{p} \cdot \vec{q}^{\prime}\right) \delta_{i j}+c_{S p}{\overrightarrow{q_{i}}}^{\prime} \hat{p}_{j}+d_{S p} \hat{p}_{i} \hat{p}_{j}\left(\vec{q}^{\prime} \cdot \hat{p}\right) \\
+ & a_{P s} \hat{p}_{i} \vec{p}_{j}{ }^{\prime}+b_{P s}\left(\hat{p} \cdot \vec{p}^{\prime}\right) \delta_{i j}+c_{P s} \vec{p}_{i}{ }^{\prime} \hat{p}_{j}+d_{P s} \hat{p}_{i} \hat{p}_{j}\left(\vec{q}^{\prime} \cdot \hat{p}\right),
\end{aligned}
$$




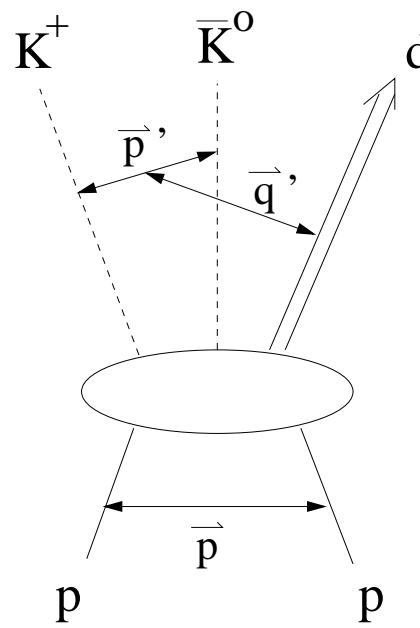

a)

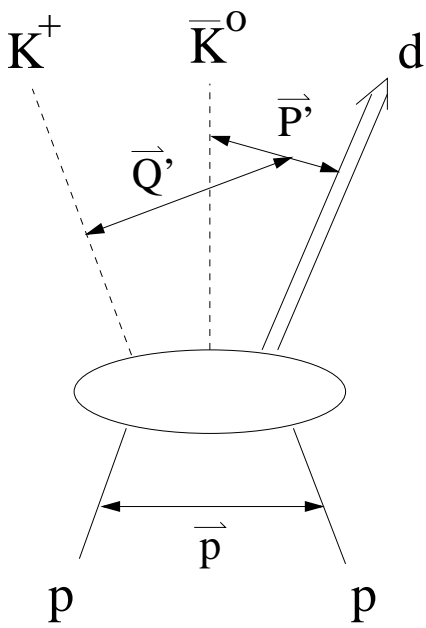

b)

Fig. 16. Illustration of the coordinate system used in the analysis for the reaction $p p \rightarrow d \bar{K}^{0} K^{+}$.

where capital letters in the amplitude label indicate the partial wave of the $\bar{K} K$ system and small letters that of the deuteron with respect to the $\bar{K} K$ system.

Once the individual terms in the amplitude are identified, it is straightforward to express the $C_{i}$ defined in sec. 4.1.1 in terms of them. We find, for example,

$$
\begin{aligned}
C_{0}^{q^{\prime}} & =\frac{1}{2}\left(\left|a_{S p}\right|^{2}+\left|c_{S p}\right|^{2}\right), \\
C_{1}^{q^{\prime}} & =\left|b_{S p}\right|^{2}+\frac{1}{2}\left|b_{S p}+d_{S p}\right|^{2}+\operatorname{Re}\left[a_{S p}^{*} c_{S p}+\left(a_{S p}+c_{S p}\right)^{*}\left(b_{S p}+d_{S p}\right)\right], \\
C_{2} & =a_{S p}^{*} a_{P s}+c_{S p}^{*} c_{P s} .
\end{aligned}
$$

A fit to the experimental data revealed that, within the experimental uncertainty, $C_{0}^{p^{\prime}}$ is compatible with zero. Thus, given the previous formulas, both $a_{P s}$ and $c_{P s}$ have to vanish individually.

Based on the strongly populated $\bar{K} K s$-waves in Ref. [66] it was argued that the reaction $p p \rightarrow d \bar{K}^{0} K^{+}$is governed by the production of the $a_{0}^{+}$. In Ref. [81], however, it was argued that the strong $\bar{K} d$ interaction caused by the proximity of the $\Lambda(1405)$ resonance should play an important role as well. This FSI should enhance the $\bar{K} d s$-wave. We now want to calculate the contribution of this partial wave relative to the $\bar{K} d p$-wave based on the amplitudes given 
in table 3. This will illustrate a further strength of the amplitude method, for within this scheme changing the coordinate system is trivial. The coordinate system suited to study resonances in the $\bar{K} K$ system and in the $\bar{K} d$ system are illustrated in the left and right panels of Fig. 16. All we need to do now is express the vectors that appear in Eq. (30) in terms of $\vec{Q}^{\prime}$ and $\vec{P}^{\prime}$. We find

$$
\vec{q}^{\prime}=\vec{P}^{\prime}-\alpha \vec{Q}^{\prime} \quad \text { and } \quad \vec{p}^{\prime}=\frac{1}{2}\left((2-\alpha) \vec{Q}^{\prime}+\vec{P}^{\prime}\right)
$$

where $\alpha=m_{d} /\left(m_{d}+m_{\bar{K}}\right)$. Obviously, the squared amplitude expressed in the new coordinates reveals the same structure as Eq. (30):

$$
\begin{aligned}
|\overline{\mathcal{M}}|^{2}=B_{0}^{Q^{\prime}} Q^{\prime 2}+B_{0}^{P^{\prime}} P^{\prime 2}+B_{1}^{Q^{\prime}} & \left(\overrightarrow{Q^{\prime}} \cdot \hat{p}\right)^{2}+B_{1}^{p^{\prime}}\left(\overrightarrow{P^{\prime}} \cdot \hat{p}\right)^{2} \\
& +B_{2}\left(\overrightarrow{P^{\prime}} \cdot \overrightarrow{Q^{\prime}}\right)+B_{3}\left(\overrightarrow{P^{\prime}} \cdot \hat{p}\right)\left(\overrightarrow{Q^{\prime}} \cdot \hat{p}\right)
\end{aligned}
$$

where the coefficients appearing can be expressed in terms of the $C$ coefficients of Eq. (30) so that, for example,

$$
\begin{aligned}
& B_{0}^{Q^{\prime}}=\frac{(2-\alpha)^{2}}{4} C_{0}^{q^{\prime}}+\alpha^{2} C_{0}^{k^{\prime}}-\frac{\alpha(2-\alpha)}{2} \frac{1}{2} C_{2}, \\
& B_{1}^{Q^{\prime}}=\frac{(2-\alpha)^{2}}{4} C_{1}^{q^{\prime}}+\alpha^{2} C_{1}^{k^{\prime}}-\frac{\alpha(2-\alpha)}{2} \frac{1}{2} C_{3} .
\end{aligned}
$$

With these expressions at hand it is easy to verify, that $K \bar{K} s$-waves contribute to $83 \%$ to the total cross section, whereas $\bar{K} d s$-waves contribute to $54 \%$ only [82]. Here we used the total $s$-wave strength for $\bar{K} K,\left(C_{0}^{q^{\prime}}+(1 / 3) C_{1}^{q^{\prime}}\right)$, and the total $s$-wave strength for $\bar{K} d,\left(B_{0}^{Q^{\prime}}+(1 / 3) B_{1}^{Q^{\prime}}\right)$, as a measure of the strength of the partial waves.

As we do not see a significant population of the $\bar{K} d s$-wave, it appears that the $\Lambda(1405)$ does not play an essential role in the reaction dynamics of $p p \rightarrow$ $d \bar{K}^{0} K^{+}$close to threshold in contrast to the $a_{0}^{+}$.

\subsection{Spin Cross Sections}

As early as 1963, Bilenky and Ryndin showed [83], that from the spin correlation coefficients that can be extracted from measurements with polarized 
Table 7

List of the lowest partial waves in the final state that contribute to the individual spin cross sections. Capital letters denote the baryon-baryon partial waves whereas small letters that of the meson with respect to the baryon-baryon system.

\begin{tabular}{|c|c|c|}
\hline${ }^{2 S+1} \sigma_{m}$ & \multicolumn{2}{|c|}{ possible final states for $p p \rightarrow b b^{\prime} x$} \\
& $S l_{x}$ & $P l_{x}$ \\
\hline${ }^{1} \sigma_{0}$ & ${ }^{3} S_{1} p$ & ${ }^{3} P_{j} s,{ }^{1} P_{1} s$ \\
${ }^{3} \sigma_{0}$ & ${ }^{1} S_{0} s,{ }^{1} S_{0} d,{ }^{3} S_{1} d$ & ${ }^{3} P_{j} p,{ }^{1} P_{1} p$ \\
${ }^{3} \sigma_{1}$ & ${ }^{3} S_{1} s,{ }^{1} S_{0} d,{ }^{3} S_{1} d$ & ${ }^{3} P_{j} p,{ }^{1} P_{1} p$ \\
\hline
\end{tabular}

beam and target, the cross section can be separated into pieces that stem from different initial spin states. Their results were recently re-derived [84] and the formalism was generalized to the differential level in Ref. [85]. With these socalled spin cross sections it can easily be demonstrated how the use of spin observables enable one to filter out particular aspects of a reaction. We begin this subsection with a derivation of the spin cross sections using the amplitude method of the previous subsection and then use the reaction $\vec{p} \vec{p} \rightarrow p n \pi^{+}$as an illustrative example.

Since we have the amplitude decomposition of the individual observables given in Eqs. (49)-(54), one easily finds

$$
\begin{array}{r}
\sigma_{0}\left(1-A_{x x}-A_{y y}-A_{z z}\right)=|H|^{2}+|A|^{2}=:{ }^{1} \sigma_{0}, \\
\sigma_{0}\left(1+A_{x x}+A_{y y}-A_{z z}\right)=\left|Q_{z}\right|^{2}+\left|B_{z n}\right|^{2}=:{ }^{3} \sigma_{0}, \\
\sigma_{0}\left(1+A_{z z}\right)=\frac{1}{2}\left(\left|Q_{x}\right|^{2}+\left|Q_{y}\right|^{2}+\left|B_{x n}\right|^{2}+\left|B_{y n}\right|^{2}\right)=:{ }^{3} \sigma_{1},
\end{array}
$$

where the assignment of the various spin cross sections ${ }^{(2 S+1)} \sigma_{M_{S}}$, with $S\left(M_{S}\right)$ the total spin (projection of the total spin on the beam axis) of the initial state can be easily confirmed from the definition of the amplitudes in Eq. (48).

As was shown in section 1.6, in case of two nucleon initial or final states restrictive selection rules apply. For example, for $p p$ final states the isospin of the final $N N$ system is 1 and therefore states with even (odd) angular momentum have total spin 0 (1). From Table 7 it thus follows, that the $p p S_{-}$ 

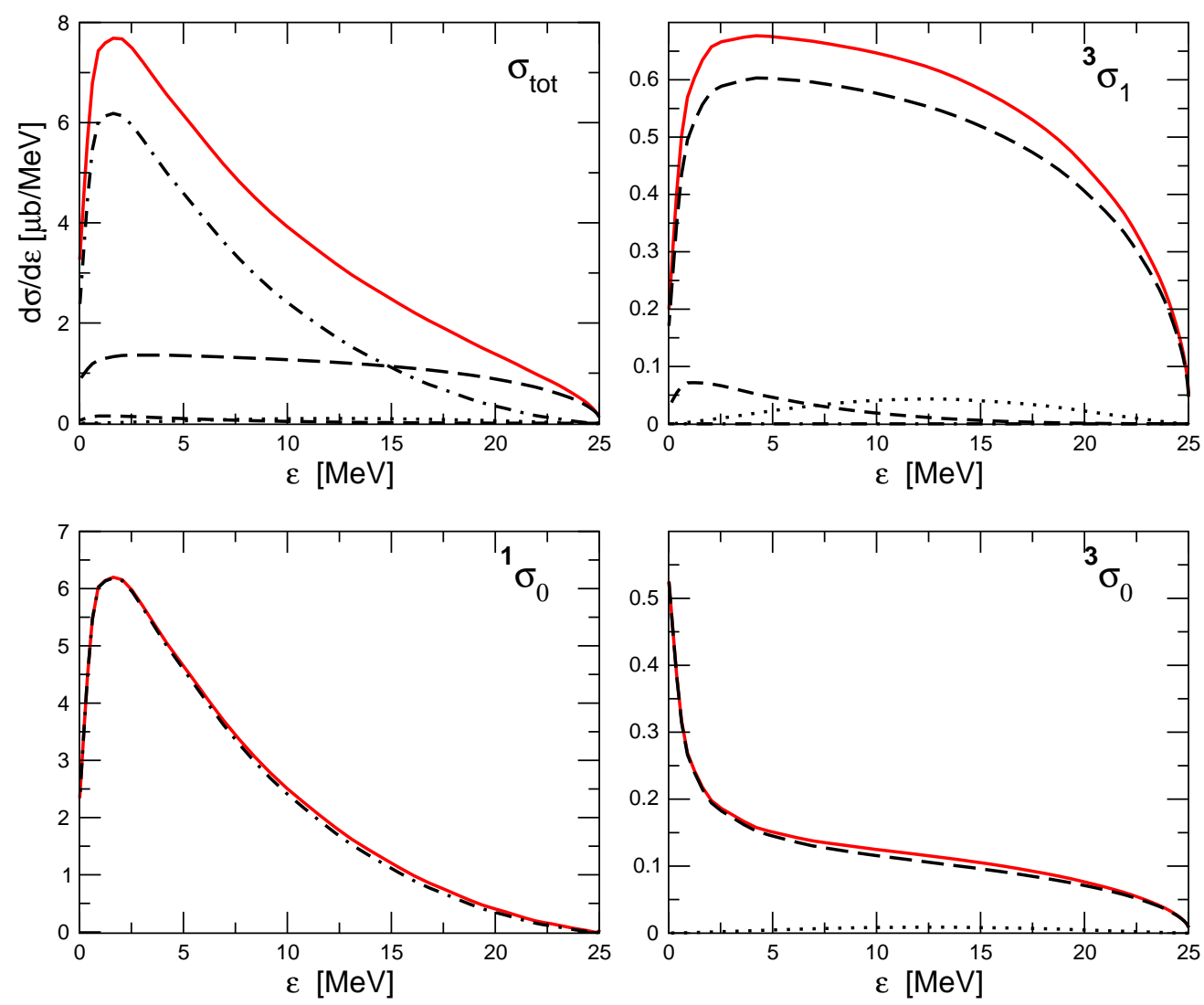

Fig. 17. Demonstration of the selectivity of the spin cross sections. Shown are the spectra of the reaction $\vec{p} \vec{p} \rightarrow p n \pi^{+}$as a function of $\epsilon$ for an excess energy of 25 $\mathrm{MeV}$. In each panel the solid line shows the full result for the corresponding cross section, the dot-dashed, long-dashed, dashed, and dotted lines show the Sp, Ss, Sd, and Pp contribution respectively. The curves are from the model of Ref. [71].

wave in connection with a meson $s$-wave contributes only to ${ }^{3} \sigma_{0}$. This example of how spin observables can be used to filter out particular final states was used previously in sec. 2 (c.f. discussion to Fig. 6).

For illustrative purposes we show in Fig. 17 the spectra for the various cross sections for the reaction $\vec{p} \vec{p} \rightarrow p n \pi^{+}$, as a function of the relative energy of the nucleon pair in the final state. The curves correspond to the model of Ref. [71] that very well describes the available data in the $\pi^{+}$production channel. The model is described in detail in sec. 6. In the upper left panel the unpolarized cross section is shown. It is dominated by the $S p$ final state (the dominant transition is ${ }^{1} D_{2} \rightarrow{ }^{3} S_{1} p$ ), and from this spectrum alone it would be a hard task to extract information on final states other than the ${ }^{3} S_{1} N N$ state. This 


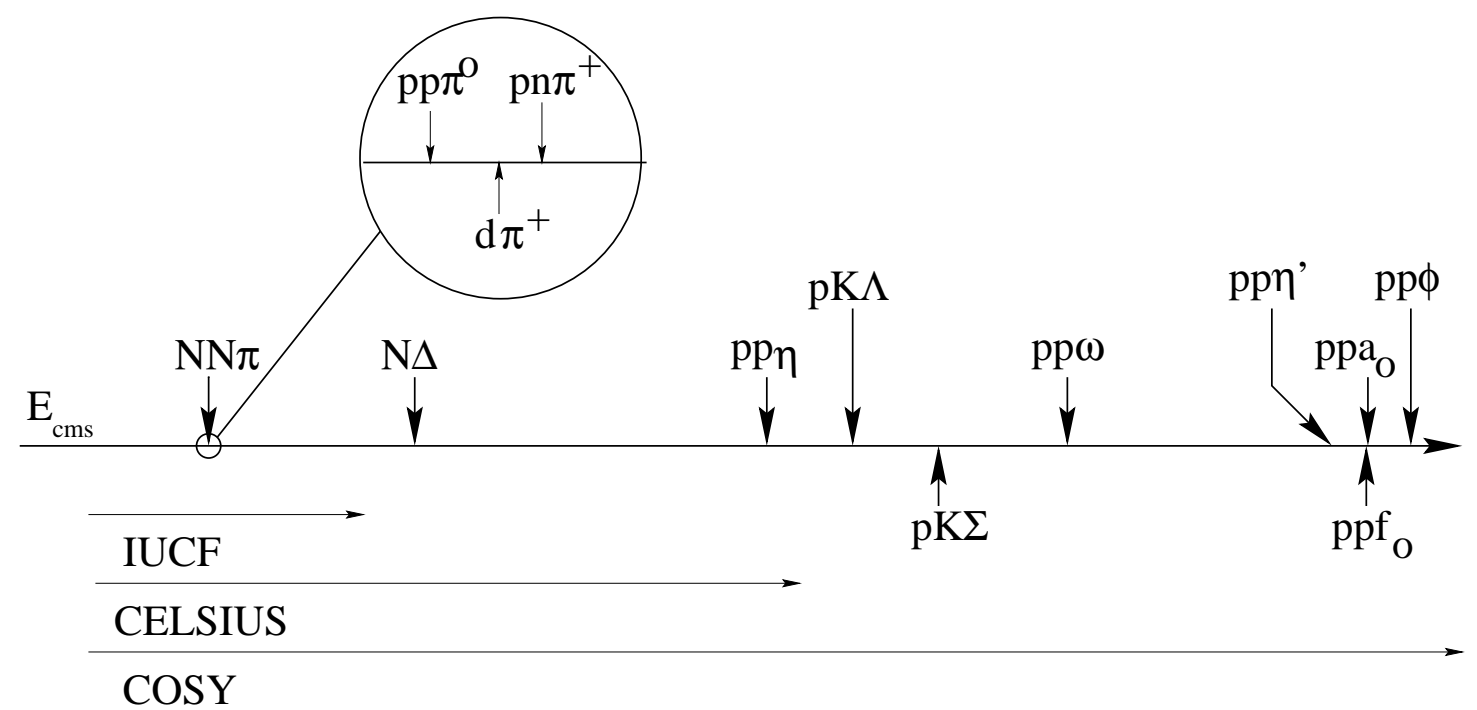

Fig. 18. The lowest meson production thresholds for single meson production in proton-proton collisions together with the corresponding energy ranges of the modern cooler synchrotrons.

can be clearly seen by the similarity of the shapes of the dot-dashed line and the solid line. The spin cross sections, however, allow separation of the spin singlet from the spin triplet initial states. Naturally ${ }^{1} \sigma_{0}$ (lower left panel of Fig. 17 ) is now saturated by the $S p$ final state, but in ${ }^{3} \sigma_{0}$ and ${ }^{3} \sigma_{1}$ other structures appear: the former is now dominated by the transition ${ }^{3} P_{0} \rightarrow{ }^{1} S_{0} s$ and the latter by ${ }^{3} P_{1} \rightarrow{ }^{3} S_{1} s$.

\subsection{Status of Experiment}

In this presentation we will be rather brief on details about current as well as planned experiments, as this subject was already covered in recent reviews $[1,2]$. Here we only wish to give a brief list of observables and reactions that are measured already or are planned to be measured in nucleon-nucleon and nucleon-nucleus induced reactions.

In the case of pion production, measurements with vector- and tensorpolarized deuteron and vector-polarized proton targets and polarized proton beams have been carried out at IUCF [134].

Because of good $4 \pi$ detection of photons and charged particles, CELSIUS, at 
Table 8

List of observables measured for various $N N \rightarrow N N x$ channels for excess energies up to $Q=40 \mathrm{MeV}$.

\begin{tabular}{|c|c|c|c|c|c|}
\hline channel & $\sigma_{t o t}$ & $d \sigma / d \Omega$ & $d \sigma / d m$ & $A_{o i}$ & $A_{i j}$ \\
\hline$p p \rightarrow p p \pi^{0}$ & {$[43,44,86-89]$} & {$[90,88,87]$} & [87-89] & {$[89,47]$} & {$[47]$} \\
\hline$p p \rightarrow p n \pi^{+}$ & [91-93] & {$[92]$} & & {$[92]$} & {$[94]$} \\
\hline$p n \rightarrow p p \pi^{-}$ & {$[77]$} & {$[77,74]$} & {$[77]$} & {$[78,75]$} & \\
\hline$p p \rightarrow d \pi^{+}$ & [95-104] & {$[95,103,104]$} & - & [105-107] & [108] \\
\hline$p p \rightarrow p p \eta$ & [109-117] & {$[113,112,118]$} & {$[113,112]$} & {$[119]$} & \\
\hline$p n \rightarrow p n \eta$ & {$[33]$} & & & & \\
\hline$p n \rightarrow d \eta$ & {$[120,114]$} & & - & & \\
\hline$p p \rightarrow p p \eta^{\prime}$ & {$[117,29,121]$} & & & & \\
\hline$p p \rightarrow p K^{+} \Lambda$ & {$[122,123,46]$} & & {$[46]$} & & \\
\hline$p p \rightarrow p K^{+} \Sigma^{0}$ & {$[124]$} & & & & \\
\hline$p p \rightarrow p p \omega$ & {$[125]$} & & & & \\
\hline$p n \rightarrow d \omega$ & {$[126]$} & & - & & \\
\hline$p p \rightarrow p p \phi$ & {$[127]$} & {$[127]$} & & & \\
\hline$p p \rightarrow p p f_{0} / a_{0}$ & {$[128]$} & & & & \\
\hline$p p \rightarrow p p \pi^{+} \pi^{-}$ & [129-131] & [129-131] & [129-131] & & \\
\hline$p p \rightarrow p n \pi^{+} \pi^{0}$ & {$[131]$} & {$[131]$} & {$[131]$} & & \\
\hline$p p \rightarrow p p \pi^{0} \pi^{0}$ & [131] & {$[131]$} & [131] & & \\
\hline$p p \rightarrow p p K^{+} K^{-}$ & {$[132]$} & & & & \\
\hline$p p \rightarrow d K^{+} \bar{K}^{0}$ & {$[133]$} & {$[133]$} & {$[133]$} & & \\
\hline
\end{tabular}

least in the near future, is well equipped for studies involving $\eta$ mesons. For the production of heavier mesons, COSY, due to its higher beam energies and intensities, but due most importantly to the possibility to use polarization, is 
in a position to dominate the field during the years to come. The energy range of the different cooler synchrotrons is illustrated in Fig. 18.

In table 8 a list is given for the various $N N$ induced production reactions measured in recent years at SACLAY, TRIUMF, PSI, COSY, IUCF, and CELSIUS in the near-threshold regime $(Q<40 \mathrm{MeV})$. The corresponding references are listed as well. In the subsections to come we will discuss some examples of the kind of physics that can be studied with the various observables in the many reaction channels.

\section{Symmetries and their violation}

As was already mentioned in several places in this article, symmetries strongly restrict the allowed pattern for various observables. This leads to observable consequences. Naturally, it is therefore also straight forward to investigate the breaking of these symmetries by looking at a violation of these symmetry predictions.

Probably the most prominent example of an experiment for a storage ring is that proposed by the TRI collaboration to be performed at the COSY accelerator. The goal is to do a null experiment in order to put an upper limit on the strength of $\mathrm{T}$-odd $\mathrm{P}$-even interactions via measuring $A_{y, x z}$ in polarized proton-deuteron scattering, which should vanish if time reversal invariance holds [135]. For details we refer to Ref. [136].

\subsection{Investigation of charge symmetry breaking (CSB)}

If the masses of the up and down quark were equal, the QCD Lagrangian were invariant under the exchange of the two quark flavors. In reality these masses are not equal and also the presence of electro magnetic effects leads to small but measurable charge symmetry breaking effects. Note, the mass difference of a few MeV [137] is small compared to the typical hadronic scale of $1 \mathrm{GeV}$. Quantifying CSB effects therefore allows to extract information on the light 
quark mass differences from hadronic observables.

As should be clear from the previous paragraph, CSB is closely linked to the isospin symmetry. However, does isospin symmetry demand an independence of the interaction under an arbitrary rotation in isospin space, a system is charge symmetric, if the interaction does not change under a 180 degree rotation in isospin space. Therefore isospin symmetry or charge independence is the stronger symmetry than charge symmetry. For an introduction into the subject we refer to Ref. [5].

The advantage of reactions with only nucleons or nuclei in the initial state is, that one can prepare initial states with well-defined isospin. This is in contrast to photon induced reactions, since a photon has both isoscalar and isovector components. Therefore, in the case of meson photo- or electro-production, CSB signals can only be observed as deviations from some expected signal. (As an example of this reasoning see Ref. [138].) In the case of hadron induced reactions on the other hand, experiments can be prepared that give a nonvanishing result only in the presence of CSB. This makes the unambiguous identification of the effect significantly easier.

One complication that occurs if a CSB effect is to be extracted from the comparison of two cross sections with different charges in the final state is that of the proper choice of energy variable. Obviously, the quantity that changes most quickly close to the threshold is the phase space, so that it appears natural to compare two reaction channels that have the same phase space volume. However, due to the differences in the particle masses, this calls for different initial energies. In the case of a resonant production mechanism this might lead to effects of the same order as the effect of interest. In Ref. [139] this is discussed in detail for the reactions $p p \rightarrow d \pi^{+}$and $p n \rightarrow d \pi^{0}$.

In this report we will concentrate on the implications of CSB on observables in $N N$ and $d d$ collisions. For details of the mechanisms of CSB we refer to Ref. [5]. In the corresponding class of experiments the deuteron as well as the alpha particle play an exceptional role since as isoscalars they can act as isospin filters. 
The most transparent example of a CSB reaction is

$$
d d \rightarrow \alpha x
$$

where $x$ is some arbitrary isovector. The reaction with $x=\pi^{o}$ was recently measured at IUCF for the first time close to the threshold [140]. The initial state as well as the $\alpha$ are pure isoscalars. Thus the final state as an isovector can not be reached as long as isospin is conserved.

In addition $p n$ reactions can be used for producing clean signals of CSB. More generally, whenever a $p n$ pair has a well-defined isospin they behave as identical particles. Specifically, the differential cross section needs to be forward-backward symmetric because nothing should change if beam and target are interchanged. Any deviation from this symmetry is an unambiguous signal of CSB [5]. An experiment performed at TRIUMF recently claimed for the first time a non-vanishing forward-backward asymmetry in $p n \rightarrow d \pi^{0}[6]$. Note that not every forward-backward asymmetry in $p n$ reactions stems from isospin violation. A counter example was given at the end of section 4.3, where the differential cross section for $p n \rightarrow p p \pi^{-}$is discussed in detail. There, in contrast to the previous example, $T=0$ and $T=1$ initial states interfere.

In case of pion production in nucleon-nucleon collisions it is possible to define a convergent effective field theory (see section 6). Within this theory it is possible to relate effects of CSB in these reactions directly to the up-down quark mass difference $[5,141]$. Preliminary studies show, that the relative importance of different CSB mechanisms in the reaction $p n \rightarrow d \pi^{0}$ and $d d \rightarrow \alpha \pi^{0}$ are very different [142], and thus it should be possible to extract valuable information on the leading CSB operators from a combined analysis of the two reactions.

In the arguments given all that was used was the isovector character of the meson produced. Thus, the same experimental signals will be seen also in $p n \rightarrow d\left(\pi^{0} \eta\right)$ [143] and $d d \rightarrow \alpha\left(\pi^{0} \eta\right)$ [144]. In Ref. [67] also the analysing power was identified as a useful quantity for the extraction of the $a_{0}-f_{0}$ mixing matrix element (see also discussion in Ref. [145]). The $\left(\pi^{0} \eta\right)_{s-\text { wave }}$ is interesting especially close to the $\bar{K} K$ threshold, since it should give insight into the nature of the light scalar mesons $a_{0}(980)$ and $f_{0}(980)$ (c.f. sec. 7.5). 


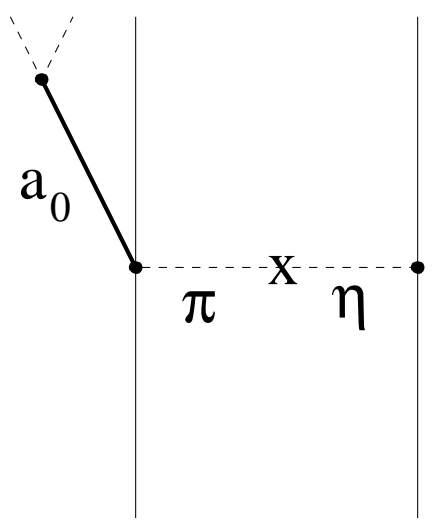

a)

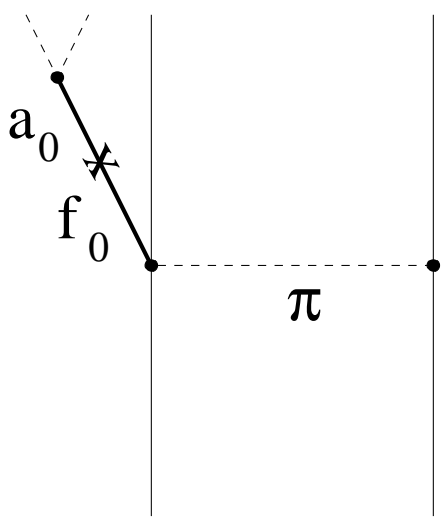

b)

Fig. 19. Illustration of different sources of charge symmetry breaking: diagram a) shows CSB in the production operator through $\pi-\eta$ mixing and diagram b) shows CSB in the propagation of the scalars. Thin solid lines denote nucleons, thick solid lines scalar mesons and dashed ones pseudoscalar mesons. The $X$ indicates the occurrence of a CSB matrix element.

Note that the $\pi \eta$ channel is the dominant decay channel of the $a_{0}$, which is an isovector-scalar particle. It should be stressed that the charge symmetry breaking signal in case of the scalar mesons is significantly easier to interpret in comparison to the case of pion production. The reason is that the two scalar resonances of interest overlap and therefore the effect of CSB as it occurs in the propagation of the scalar mesons is enhanced compared to mixing in the production operator [82]. To make this statement more quantitative we compare the impact of $f_{0}-a_{0}$ mixing in the propagation of the scalar mesons (Fig. 19b) to that of $\pi-\eta$ mixing in the production operator (Fig. 19a). We regard the latter as a typical CSB effect and thus as a reasonable order-of-magnitude estimate for CSB in the production operator. Observe that the relevant dimensionless quantity for this comparison is the mixing matrix element times a propagator (c.f. Fig. 19). In the production operator the momentum transfer - at least close to the production threshold - is given by $t=-M_{N} m_{R}$, where $m_{R}$ denotes the invariant mass of the meson system produced (or equivalently the mass of the resonance) and $M_{N}$ denotes the nucleon mass. Thus, the appearance of the $\eta$ propagator introduces a factor of about $1 / t$ into the amplitude, since $t \gg m_{\eta}^{2}$. On the other hand, the resonance propagator is given by $1 /\left(m_{R} \Gamma_{R}\right)$, as long as we concentrate on invariant masses of the outgoing meson system close to the resonance position. Here $\Gamma_{R}$ 
denotes the width of the scalar resonance. Thus we find using $\Gamma_{R}=50 \mathrm{MeV}$ [146] that the CSB in the production operator is kinematically suppressed by a factor of more than $\Gamma_{R} / M_{N} \sim 1 / 20$ as compared to CSB in the propagation of the scalars. In addition the mixing matrix element is enhanced in the case of $f_{0}-a_{0}$ mixing (c.f. sec. 7.5) and therefore it should be possible to extract the $f_{0}-a_{0}$ mixing matrix element from $N N$ and $d d$ induced reactions.

\section{The reaction $N N \rightarrow N N \pi$}

The production of pions in nucleon-nucleon collisions has a rather special role. First of all, it is the lowest hadronic inelasticity for the nucleon-nucleon interaction and thus an important test of our understanding of the phenomenology of the $N N$ interaction. Secondly, since pions are the Goldstone bosons of chiral symmetry, it is possible to study this reaction using chiral perturbation theory. This provides the opportunity to improve the phenomenological approaches via matching to the chiral expansion as well as to constrain the chiral contact terms via resonance saturation. Last but not least, a large number of (un)polarized data is available (c.f. table 8).

After a brief history, we continue this chapter with a discussion of a particular phenomenological model for pion production near the threshold, followed by a presentation of recent results from chiral perturbation theory.

\subsection{Some History}

In section 1.2 it was argued, that for the near-threshold regime the distorted wave born approximation is appropriate, and here we will concentrate on those models that work within this scheme ${ }^{19}$.

Pioneering work on pion production was done by Woodruf [147] as well as by Koltun and Reitan [31] in the 1960s. The diagrams included are shown in

\footnotetext{
${ }^{19}$ In the next section a further argument in favor of the distorted wave born approximation will be given.
} 
Figs. $2 \mathrm{a}$ and $2 \mathrm{~b}$ where, in these early approaches, the $\pi N \rightarrow \pi N$ transition amplitudes (denoted by $T$ in diagram $2 \mathrm{~b}$ ) were parameterized by the scattering lengths.

When the first data on the reaction $p p \rightarrow p p \pi^{0}$ close to the threshold were published [43,44], it came as a big surprise that the model of Koltun and Reitan [31] underestimated the data by a factor of $5-10$. This is in vast contrast to the reaction $p p \rightarrow p n \pi^{+}$reported in Ref. [91], where the discrepancy was less than a factor of 2 . On the other hand, it was shown that the energy dependence of the total cross section can be understood from that of the $N N$ FSI once the Coulomb interaction is properly included [45] (c.f. sec. 2).

Niskanen investigated whether the inclusion of the Delta isobar, as well as keeping the rather strong on-shell energy dependence of the $\pi N$ interaction, could help to improve the theoretical results for neutral pion production [148]. Although these improvements lead to some enhancement, the cross section was still missed by more than a factor of 3 .

The first publication that reported a quantitative understanding of the $p p \rightarrow$ $p p \pi^{0}$ data was that by Lee and Riska [149] and later confirmed by Horowitz et al. [150], where it was demonstrated that short range mechanisms as depicted in Fig. 2c, can give a sizable contribution. However, shortly after this discovery Hernández and Oset demonstrated, using various parameterizations for the $\pi N \rightarrow \pi N$ transition amplitude and qualitatively reproducing earlier work by Hachenberg and Pirner [151] that the strong off-shell dependence of that amplitude can also be sufficient to remove the discrepancy between the Koltun and Reitan model and the data. Gedalin et al. came to the same conclusion within a relativistic one boson exchange model [152]. In Ref. [153] the $\pi N$ amplitude needed as input for the evaluation of diagram $2 \mathrm{~b}$ was extracted from a microscopic model. Also there a significant although smaller contribution from the pion rescattering was found. Thus, in this model still some additional short range mechanism is needed.

In the succeeding years many theoretical works presented calculations for the $p p \rightarrow p p \pi^{0}$ cross section. In Refs. [154,155] covariant one boson exchange models were used in combination with an approximate treatment of the nucleon- 
nucleon interaction. Both models turned out to be dominated by heavy meson exchanges and thus give further support to the picture proposed in Ref. [149]. However, in Ref. [156] the way that the anti nucleons were treated in Refs. $[149,150,154]$ was heavily criticized: the authors argued that the anti nucleon contributions get significantly suppressed once they are included non perturbatively. It is interesting to note, that also in Bremsstrahlung the contribution from anti-nucleons in a non-perturbative treatment is significantly reduced compared to a perturbative inclusion $[157,158]$. Additional short range contributions were also suggested, namely the $\rho-\omega$ meson exchange current [159], resonance contributions [160] and loops that contain resonances [161], all those, however, turned out to be smaller compared to the heavy meson exchanges and the off-shell pion rescattering, respectively.

At that time the hope was that chiral perturbation theory might resolve the true ratio of rescattering and short range contributions. It came as a big surprise, however, that the first results for the reaction $p p \rightarrow p p \pi^{0}[162,163]$ found a rescattering contribution that interfered destructively with the direct contribution (diagram a in Fig. 2), making the discrepancy with the data even more severe. In addition, the same isoscalar rescattering amplitude also worsened the discrepancy in the $\pi^{+}$channel $[164,165]$. Some authors interpreted this finding as a proof for the failure of chiral perturbation theory in these large momentum transfer reactions $[155,166]$. Only recently was it demonstrated, that it is possible to appropriately modify the chiral expansion in order to make it capable of analysing meson production in nucleon-nucleon collisions. We will report on those studies in sec. 6.3 that in the future will certainly prove useful for improving the phenomenological approaches (c.f. subsec. 6.5).

Before we close this section a few remarks on the off-shell $\pi N$ amplitude are necessary. For this purpose we write the relevant piece of the $\pi N$ interaction $T$-matrix in the following form

$$
T_{\pi N}=-t^{(+)} \frac{1}{2} N^{\dagger} \pi \cdot \pi N+t^{(-)} \frac{1}{2 m_{\pi}} N^{\dagger} \tau \cdot(\pi \times \dot{\pi}) N
$$

where $t^{(+)}\left(t^{(-)}\right)$denote the isoscalar (isovector) component. Note that it is only the former that can contribute to the reaction $p p \rightarrow p p \pi^{020}$, for the

\footnotetext{
${ }^{20}$ This is only true if we do not include the $\Delta$ isobar explicitly, as will be discussed
} 
isospin structure of the latter changes the total isospin of the two nucleon system.

As long as we neglect the distortions due to the final and initial state interactions, what is relevant for the discussion in this paragraph is the half off-shell $\pi N$ amplitude. We thus may write $t=t\left(s, k^{2}\right)$, where $s$ denotes the invariant energy of the $\pi N$ system and $k^{2}$ is the square of the four momentum of the incoming pion. At the threshold for elastic $\pi N$ scattering $\left(s=\left(m_{\pi}+M_{N}\right)^{2}\right.$, $k^{2}=m_{\pi}^{2}$ ) we may write

$$
\begin{aligned}
t^{(+)}\left(s_{0}, m_{\pi}^{2}\right) & :=\frac{4 \pi}{3}\left(1+\frac{m_{\pi}}{M_{N}}\right)\left(a_{1}+2 a_{3}\right)=(-0.05 \pm 0.01) m_{\pi}^{-1} \\
t^{(-)}\left(s_{0}, m_{\pi}^{2}\right) & :=\frac{4 \pi}{3}\left(1+\frac{m_{\pi}}{M_{N}}\right)\left(a_{1}-a_{3}\right)=(1.32 \pm 0.02) m_{\pi}^{-1}
\end{aligned}
$$

where the $a_{2 I}$ denote the scattering lengths in the corresponding isospin channels $I$. The corresponding values were extracted from data on $\pi^{-} d$ atoms in Ref. [167]. Note that the dominance of the isovector interaction is a consequence of the chiral symmetry: the leading isoscalar rescattering is suppressed by a factor $m_{\pi} / M_{N}$ compared to the leading iso-vector contribution-the so called Weinberg-Tomozawa term [168,169]. However, it is still remarkable, that also the higher order chiral corrections are small leaving a value consistent with zero for the isoscalar scattering length. A detailed study showed, that this smallness is a consequence of a very efficient cancellation of several individually large terms that are accompanied with different kinematical factors [170]. To be concrete: to order $\mathcal{O}\left(p^{2}\right)$ one finds

$$
t^{(+)}=\frac{2}{f_{\pi}^{2}}\left(-2 m_{\pi}^{2} c_{1}+q_{0}^{\prime} k_{0}\left(c_{2}-\frac{g_{A}^{2}}{8 M_{N}}\right)+\left(q^{\prime} k\right) c_{3}\right),
$$

where $k$ and $q^{\prime}$ denote the four momentum of the initial and final pion respectively. The values for the various $c_{i}$ are given in table 9. For on-shell scattering at the threshold $\left(q^{\prime}=k=\left(m_{\pi}, \overrightarrow{0}\right)\right)$ one gets $t^{(+)}\left(s_{0}, m_{\pi}^{2}\right)=-0.24 m_{\pi}^{-1}$ using the values of Ref. [171] ${ }^{21}$. Please note, that the linear combination of the $c_{i}$ appearing above turns out to be an order of magnitude smaller than the individ-

in the next section.

${ }^{21}$ Note, this value is inconsistent with the empirical value given in Eq. (71). To come to a consistent value one has to go to one loop order as discussed in Ref. [172]. 
ual values. As a consequence, the on-shell isoscalar amplitude shows a rather strong energy dependence above threshold. It should not then come as a surprise, that the transition amplitude corresponding to Eq. (70), when evaluated in the kinematics relevant for pion production in $N N$ collisions, within chiral perturbation theory turns out to be rather large numerically $[162,163]$. For the non-covariant expression given in Eq. (72) this translates into $q^{\prime}=\left(m_{\pi}, \overrightarrow{0}\right)$ and $k=\left(m_{\pi} / 2, \vec{k}\right)$, leading to $t^{(+)}\left(\left(m_{\pi}+M_{N}\right)^{2},-M_{N} m_{\pi}\right)=0.5 m_{\pi}^{-1}$. This is why the first calculations using chiral perturbation theory found a big effect from pion rescattering - unfortunately increasing the discrepancy with the data. In Ref. [173] the tree level chiral perturbation theory calculations where repeated using a different prescription for the energy of the exchange pion ${ }^{22}$. The authors found agreement with the data, but with a sign of the full amplitude opposite to the one of the direct term.

One year earlier it was shown, that within phenomenological approaches the isoscalar transition amplitude evaluated in off-shell kinematics is also significantly different from its on-shell value. For example, in the Jülich meson exchange model it is the contribution from the iterated $\rho t$-channel exchange and the $\sigma$ exchange that are individually large but basically cancel in threshold kinematics in the isoscalar channel [153]. This cancellation gets weaker away from the threshold point. This rescattering contribution, however, turned out to interfere constructively with the direct term.

Since chiral perturbation theory as the effective field theory for low energy strong interactions is believed to be the appropriate tool to study pion reactions close to threshold, it seemed at this stage as if there were a severe problem with the phenomenology. However, as was shown in section 4.3, there are observables that are sensitive to the sign of the $s$-wave $p p \rightarrow p p \pi^{0}$ amplituderelative to a $p$-wave amplitude that is believed to be under control - and the experimental results $[74,75,77,78]$ agree with the sign as given by the phenomenological model (c.f. Fig. 15) .

Does this mean that chiral perturbation theory is wrong or not applicable? No. As we will discuss in the subsequent sections, it was demonstrated recently

${ }^{22}$ This prescription was later criticized in Ref. [174]. 
that the chiral counting scheme needs to be modified in the case of large momentum transfer reactions. No complete calculation has been carried out up to now, but intermediate results look promising for a consistent picture to emerge in the years to come. The insights gained so far from the effective field theory studies call also for a modification of the phenomenological treatment. This will be discussed in detail in section 6.5 .

\subsection{Phenomenological approaches}

As stressed in the previous section, the number of phenomenological models for pion production is large. For definiteness in this section we will focus on one particular model, namely that presented in Refs. [71,73,153], mainly because it incorporates most of the mechanisms proposed in the literature for pion production in nucleon-nucleon collisions, its ingredients are consistent with the data on $\pi N$ scattering, and it is the only model so far whose results have been compared to the polarization data recently measured at IUCF $[47,94,108]$.

The model is the first attempt to treat consistently the $N N$ as well as the $\pi N$ interaction for meson production reactions close to the threshold: both were taken from microscopic models (described in Refs. [175] and [176] for the $N N$ and the $\pi N$ interaction, respectively). These were constructed from the same effective Lagrangians consistent with the symmetries of the strong interaction and are solutions of a Lippman-Schwinger equation based on time-ordered perturbation theory. Although not all parameters and approximations used in the two systems are the same, this model should still be viewed as a benchmark calculation for pion production in $N N$ collisions within the distorted wave Born approximation. We will start this section with a description of the various ingredients of the model and then present some results.

\subsubsection{The $N N$ interaction}

A typical example of a so-called realistic model for $N N$ scattering is the Bonn potential [26]. The model used for the $N N$ distortions in the final and initial states is based on this model, where a pseudo-potential is constructed based 


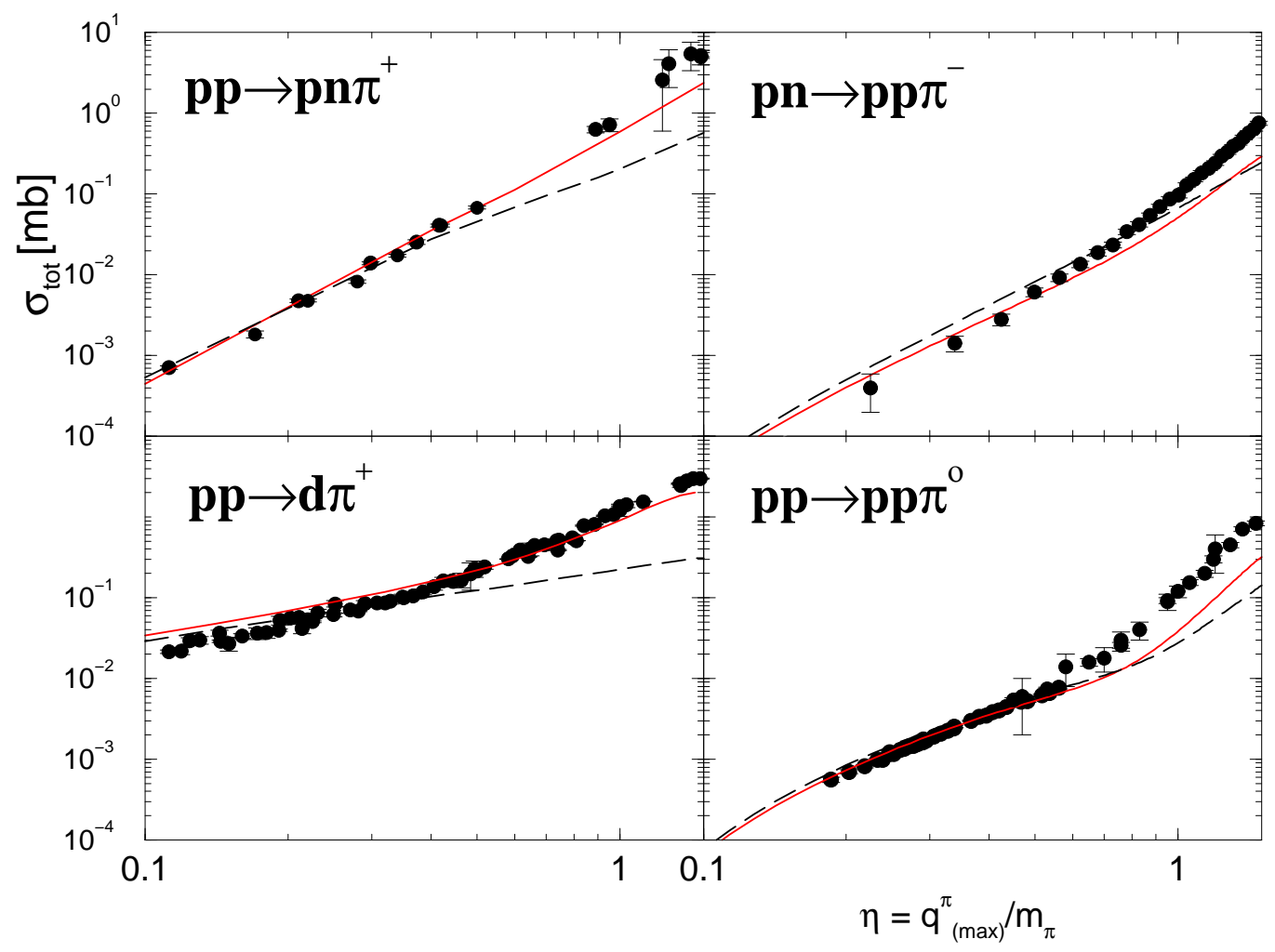

Fig. 20. Comparison of the model predictions of Ref. [73] to the data. The references for the experimental data can be found in table 8. The solid lines show the results of the full model; the dashed line shows the results without the $\Delta$ contributions.

on the $t$-channel exchanges of all established mesons below one $\mathrm{GeV}$ in mass between both nucleons and Delta isobars.

The interaction amongst the various dynamical fields in the model derived from the following Lagrange densities:

$$
\begin{aligned}
\mathcal{L}_{N N \pi} & =\frac{f_{N N \pi}}{m_{\pi}} \bar{\psi} \gamma_{5} \gamma_{\mu} \boldsymbol{\tau} \cdot \partial^{\mu} \boldsymbol{\pi} \psi \\
\mathcal{L}_{N N \rho} & =g_{N N \rho} \bar{\psi} \gamma_{\mu} \boldsymbol{\tau} \cdot \boldsymbol{\rho}^{\mu} \psi+\frac{f_{N N \rho}}{4 M_{N}} \bar{\psi} \sigma_{\mu \nu} \boldsymbol{\tau} \cdot\left(\partial^{\mu} \boldsymbol{\rho}^{\nu}-\partial^{\nu} \boldsymbol{\rho}^{\mu}\right) \psi \\
\mathcal{L}_{N N \omega} & =g_{N N \omega} \bar{\psi} \gamma_{\mu} \omega^{\mu} \psi \\
\mathcal{L}_{N N \sigma} & =g_{N N \sigma} \bar{\psi} \sigma \psi \\
\mathcal{L}_{N N a_{0}} & =g_{N N a_{0}} \bar{\psi} \boldsymbol{\tau} \cdot \boldsymbol{a}_{\mathbf{0}} \psi \\
\mathcal{L}_{N \Delta \pi} & =\frac{f_{N \Delta \pi}}{m_{\pi}} \bar{\psi} \vec{T} \cdot \partial^{\mu} \boldsymbol{\pi} \psi_{\mu}+\text { h.c. } \\
\mathcal{L}_{N \Delta \rho} & =i \frac{f_{N \Delta \rho}}{m_{\pi}} \bar{\psi} \gamma_{5} \gamma_{\mu} \vec{T} \cdot\left(\partial^{\mu} \boldsymbol{\rho}^{\nu}-\partial^{\nu} \boldsymbol{\rho}^{\mu}\right) \psi_{\nu}+\text { h.c. }
\end{aligned}
$$



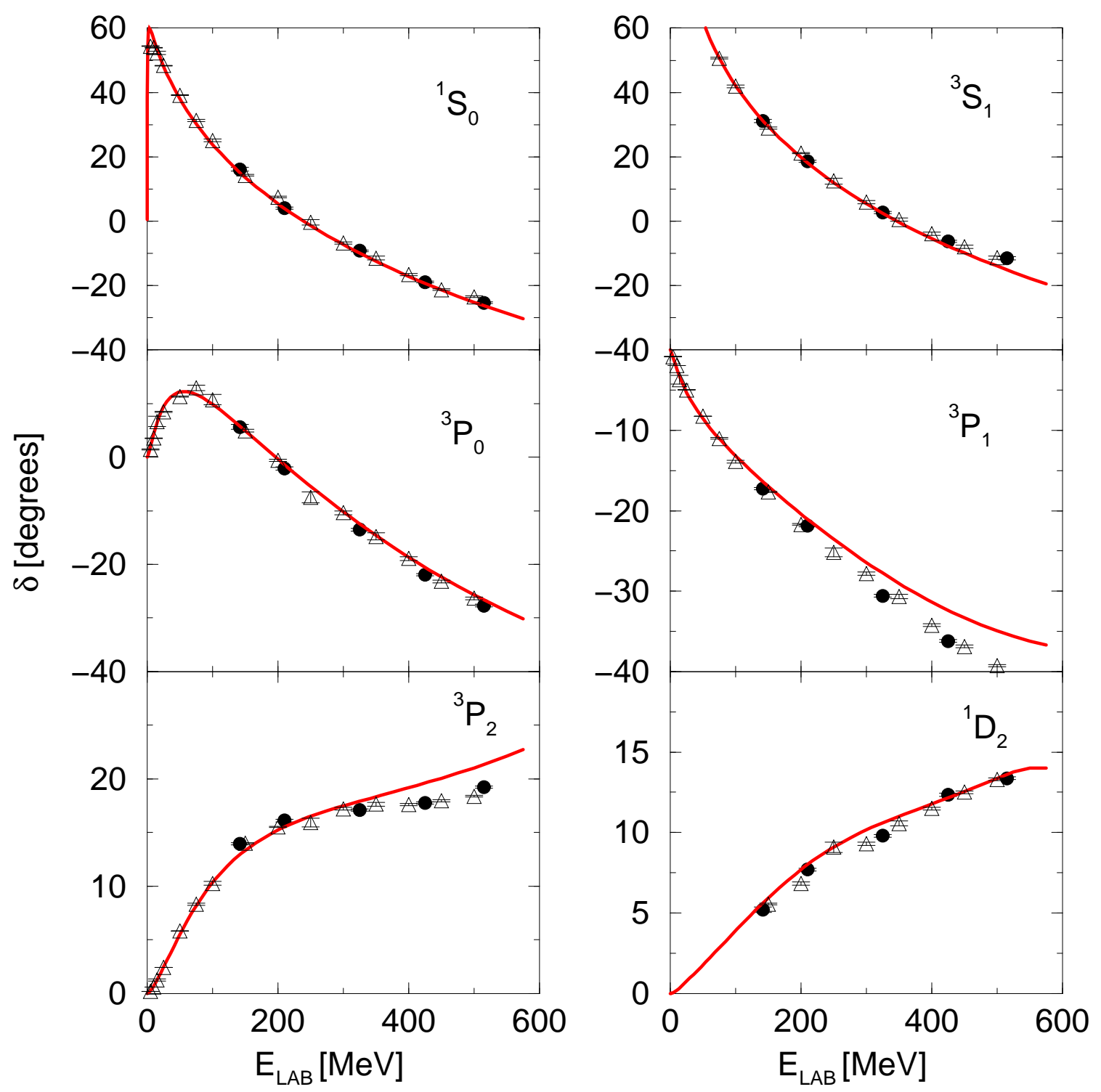

Fig. 21. The NN phase shifts for the model of Ref. [175] in the energy range relevant for pion production. The pion production threshold is at $E_{L A B}=286 \mathrm{MeV}$. The experimental data are from Refs. [177] (triangles) and [61] (circles).

Note: The particle called $\delta$ in the original Bonn publication [26] is nowadays called $a_{0}$. The operator $\vec{T}$ as well as the fields are defined in Ref. [26]. Note that there is no tensor coupling for the $\omega N N$ vertex given for the fit to the elastic $N N$ scattering data did not need any such coupling.

There is a difference between the nucleon-nucleon model used [175] and the Bonn potential [26]. The original Bonn model has an energy dependent interaction, for it keeps the full meson retardation in the intermediate state. 
This, however, leads to technical problems, once the model is to be evaluated above the pion production threshold due to the occurrence of three body singularities. In Ref. [24] those singularities were handled by solving the dynamical equations in the complex plane. Unfortunately, this method is not useful for the application in a distorted wave Born approximation. Instead we used a model based on the so-called folded diagram formalism developed in Ref. [178]. This formalism, worked out to infinite order, is fully equivalent to time-ordered perturbation theory. When truncated at low order, however, it leads to energy independent potentials that can formally be evaluated even above the pion production threshold. In addition, the model of Ref. [175] is constructed as a coupled-channel model including the $N N$ as well as the $N \Delta$ and $\Delta \Delta$ channels. This enables us to treat the $\Delta$ isobar on equal footing with the nucleons.

The resulting phase shifts are shown in Fig. 21. Note that the model parameters were adjusted to the phase shifts below the pion production threshold only, which is located at $E_{L A B}=286 \mathrm{MeV}$. Fig. 21 thus clearly illustrates that using this model for the $N N$ interaction is indeed justified and we may conclude that - at least up to laboratory energies of $600 \mathrm{MeV}$-the $N N$ phenomenology is well understood.

\subsubsection{The $\pi N$ model}

The $\pi N$ interaction that enters in the pion rescattering diagrams can be taken from a meson exchange model as well [179]. This allows a consistent treatment of the meson and nucleon dynamics. It should be stressed that this is the precondition for comparing the results of the phenomenological model to those of chiral perturbation theory, as we will do below. In addition, since we also want to include the rescattering diagram in partial waves higher than the $s^{-}$ wave, after a fit to the $\pi N$ data the pole contributions (nucleon and Delta) need to be removed from the amplitudes in order to avoid double counting with the direct production. This is possible only within a microscopic model.

The main features of the $\pi N$ model of Ref. [179] are that it is based on an effective Lagrangian consistent with chiral symmetry to leading order and that 


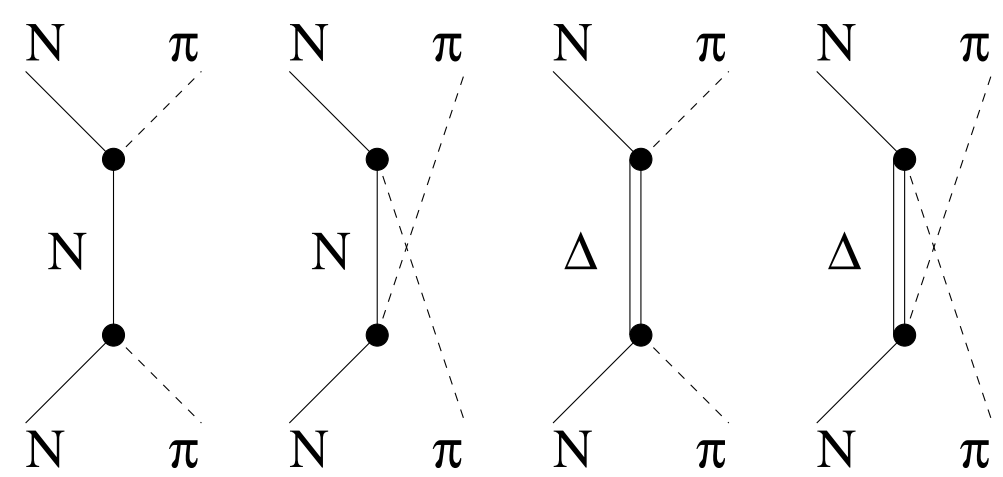

a)

b)

c)

d)

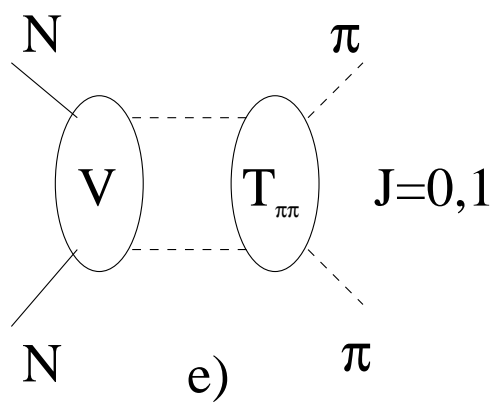

Fig. 22. Contributions to the potential of the model of Ref. [179].

the $t$-channel exchanges in the isovector $(\rho)$ and isoscalar $(\sigma)$ channel are constructed from dispersion integrals ${ }^{23}$. For details on how the $t$-channel exchanges are included we refer to Ref. [179]. The diagrams that enter the potential are displayed in Fig. 22. This potential is then unitarized with a relativistic Lippmann-Schwinger equation - in complete analogy to the nucleon-nucleon interaction.

Within this model, at tree level the isoscalar and isovector $\pi N$ interaction are give by the corresponding $t$-channel exchanges. In the latter case the unitarization does not have a big influence in the near-threshold regime, and thus also the isovector scattering length is governed by the tree level $\rho$-exchange. The famous KFSR relation [181,182], that relates the couplings of the $\rho$-meson to pions and nucleons to the coupling strength of the Weinberg-Tomozawa term, is a consequence of this. On the other hand, for the isoscalar $\pi N$ interaction the effects of the unitarization are large and lead to an almost complete can-

\footnotetext{
$\overline{23}$ There are ambiguities in how to extrapolate the results of the dispersion integrals to off-shell kinematics. This issue is discussed in detail in Ref. [180].
} 

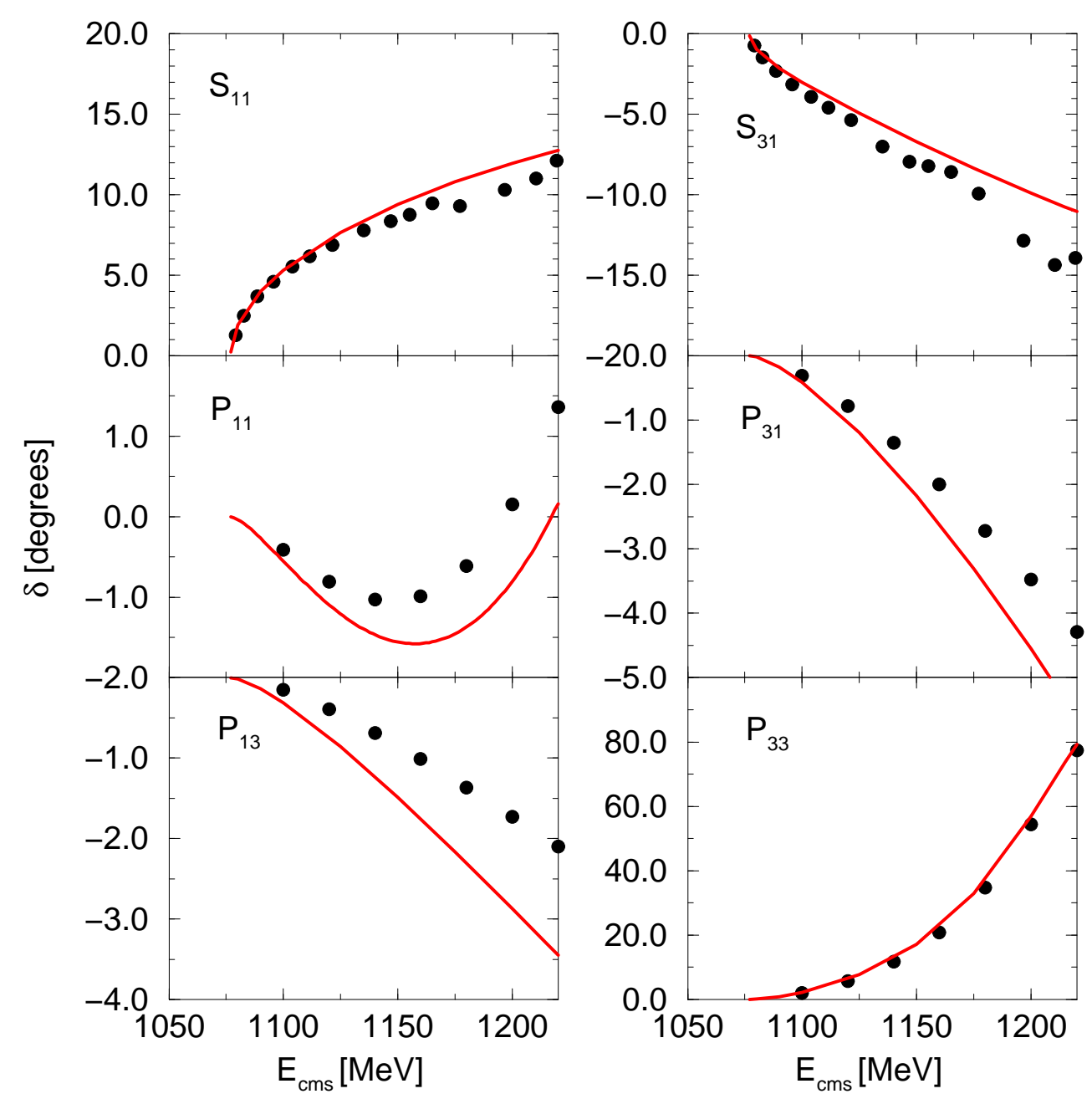

Fig. 23. The $\pi N$ phase shifts for the model of Ref. [179] in the energy range relevant for pion production. The experimental data are from Refs. [183,184]. Note the different scales of the various panels.

cellation of the isoscalar potential with the iterated $\rho$-exchange in the near threshold regime. As one moves away from the threshold value this cancellation gets weaker leading to the strong variation of the off-shell isoscalar $\pi N$ $T$-matrix mentioned at the end of sec. 6.1.

In Figure 23 the results for the model of Ref. [179] are compared to the data of Refs. [183,184]. As one can see the model describes the data well, especially in the most relevant partial waves: $S_{11}, S_{31}$ and $P_{33}$. 


\subsubsection{Additional short range contributions and model parameters}

As was stressed above, a large class of additional mechanisms was suggested in the literature to contribute significantly to pion production in nucleon-nucleon collisions. Since they are all of rather short range and mainly influence the production of $s$-wave pions, in this work only a single diagram was included (heavy meson exchange through the $\omega-$ Fig. 2c) to parameterize these various effects. Consequently, the strength of this contribution was adjusted to reproduce the total cross section of the reaction $p p \rightarrow p p \pi^{0}$ close to the production threshold. The short-range contributions turn out to contribute about $20 \%$ to the amplitude. After this is done, all parameters of the model are fixed.

\subsubsection{Results}

The results of the model presented have already appeared several times in this report (Figs. 13,14,15,17,20,24) - mainly for illustrative purposes - and they are discussed in detail in Refs. [73,71].

Overall the model is rather successful in describing the data, given that only one parameter was adjusted to the total cross section for low energy neutral pion production (see sec. 6.2.3). One important finding is that the sign of the $s$-wave neutral pion production seems to be in accord with experiment, as is illustrated in Fig. 15 (c.f. corresponding discussion in sec. 4.3), in contrast to the early calculations using chiral perturbation theory. As we will see in sec. 6.3 , today we know that those early calculations using effective field theory were incomplete.

The most striking differences appear, however, for double polarization observables in the neutral pion channel, as shown in Figs. 13 and 14. As a general pattern the amplitudes seem to be of the right order of magnitude, but show a wrong interference pattern. To actually allow a detailed comparison of the model and data a partial wave decomposition of both is necessary. Work in this direction is under way.

It is striking that for charged pion production the pattern is very different, for

here almost all observables are described satisfactorily. In Fig. 24 the results of 
the model for charged and neutral pion production are compared to the data for a few observables. In contrast to the neutral channel, the charged pion production is completely dominated by two transitions, namely ${ }^{3} P_{1} \rightarrow{ }^{3} S_{1} s$, which is dominated by the isovector pion rescattering, and ${ }^{1} D_{2} \rightarrow{ }^{3} S_{1} p$, which governs the cross section especially in the regime of the Delta resonance. The prominence of the Delta resonance is a consequence of the strong transition ${ }^{1} D_{2}(N N) \rightarrow{ }^{5} S_{2}(N \Delta)$ that even shows up as a bump in the $N N$ phase shifts (see Fig. 21). This effect was first observed long ago and is well known (see, e.g., discussion in Ref. [185]). As a consequence, the $N N \rightarrow N \Delta$ transition potential should be rather well constrained by the $N N$ scattering data and is not the case for all the many transitions relevant in case of the neutral pion production, where the $S p$ final state is not allowed due to selection rules (see sec. 1.6). One might therefore hope to learn more about the $\Delta N$ interaction from the pion production data.

One can also ask how well we know the production operator. Fortunately, at the pion production threshold it is still possible to analyze meson production in $N N$ collisions within effective field theory. This analysis will give deeper insight into the production dynamics, as will be explained in the following section. The two approaches are then compared in sec. 6.5.

\subsection{Chiral perturbation theory}

The phenomenological approaches, such as the one described in the previous section, lack a systematic expansion. Thus it is neither possible to estimate the associated model uncertainties nor to systematically improve the models. On the other hand, for various meson production reactions the phenomenological approaches proved to be quite successful. One might therefore hope that effective field theories will give insights into why the phenomenology works, as was first stressed by Weinberg [186].

A first attempt to construct - model independently - the transition amplitude $N N \rightarrow N N \pi$ was carried out almost 40 years ago [187-189]. The authors tried to relate what is known about nucleon-nucleon scattering to the pro- 

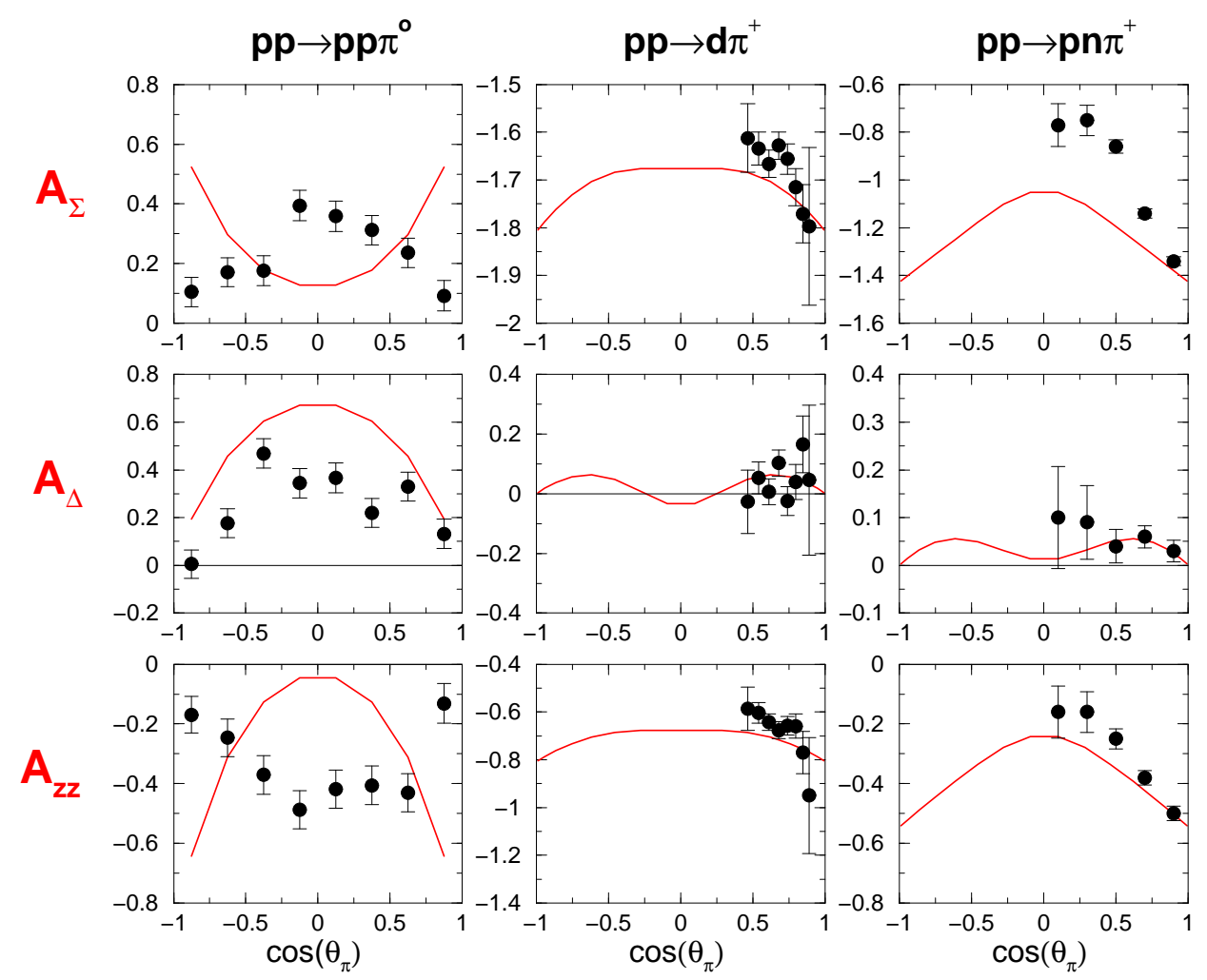

Fig. 24. Comparison of the model predictions to the data taken from Ref. [47] $\left(p p \rightarrow p p \pi^{o}\right), \operatorname{Ref}$. [108] $\left(p p \rightarrow d \pi^{+}\right)$and Ref. [94] $\left(p p \rightarrow p n \pi^{+}\right)$.

duction amplitude via the low energy theorem of Adler and Dothan [190], which is a generalization of the famous Low theorem for conserved currents [191] to partially conserved currents via the PCAC relation (see, e.g., Ref. [192]). However, it soon was realized that the extrapolation from the chiral limit $\left(m_{\pi}=0\right)$ to the physical point can change the hierarchy of different diagrams. The reason for the non-applicability of soft radiation theorems to meson production close to the threshold is easy to see: a necessary condition for the soft radiation theorem to be applicable is that the energy emitted is significantly smaller than the typical energy scale, that characterizes variations of the nuclear wave function. In the near-threshold regime, however, the scale of variation is set by the inverse of the $N N$ scattering lengths - thus a soft radiation theorem of the type of Low or that of Adler and Dothan could only be applicable to meson production if $m_{\pi} \ll 1 /\left(M_{N} a^{2}\right)$. In reality, however, the pion mass exceeds the energy scale introduced by inverse $N N$ scattering length by more than two orders of magnitude. The range of applicability of soft radiation theorems is discussed in Ref. [193] in a different context. 
More recent analyses, however, show that in the case of pion production it is indeed possible to define a convergent effective field theory that allows a systematic study of the structure of the production operator. As we will show, most of the diagrams included in the Koltun and Reitan model [31] are indeed the leading operators in pion production. In addition this study will show

- that the use of the distorted wave born approximation is justified

- why neutral pion production is the more problematic case

- the importance of loop contributions

- that there is a close connection between pion production in nucleon-nucleon collisions and the three nucleon problem.

The problem with strong interaction phenomena is their non-perturbative nature with respect to the coupling constants. To construct a controlled expansion it is necessary, to identify a small expansion parameter. In general this is only possible for a limited energy range. The conditio sine qua non for constructing an effective field theory for any system is the separation of scales characteristic for the system. Once the - in this context light-scales are identified, one treats them dynamically, while all the dynamics that are controlled by the heavy scales are absorbed in contact interactions. As long as the relevant external momenta and energies are such that structures of the size of the inverse of the heavy scale can not be resolved, this procedure should always work. Weinberg [194] as well as Gasser and Leutwyler [195] have shown that this general idea works even when loops need to be included.

In case of low energy pion physics it is the chiral symmetry that provides both preconditions for the construction of an effective field theory, in that it forces not only the mass of the pion $m_{\pi}$, as the Goldstone boson of the chiral symmetry breaking, to be low, but also the interactions to be weak, for the pion needs to be free of interactions in the chiral limit for vanishing momenta. The corresponding effective field theory is called chiral perturbation theory $(\chi P T)$ and was successfully applied to meson-meson [196] scattering. Treating baryons as heavy allows straightforward extension of the scheme to meson-baryon [197] as well as baryon-baryon [198-201] systems. In all these references the expansion parameter used was $p / \Lambda_{\chi} \sim p /\left(4 \pi f_{\pi}\right) \sim p / M_{N}$, where 
$\Lambda_{\chi}$ denotes the chiral symmetry breaking scale, $f_{\pi}$ the pion decay constant and $M_{N}$ the nucleon mass. Recently it was shown that also the Delta isobar can be included consistently in the effective field theory [202]. The authors treated the new scale, namely the Delta nucleon mass splitting $\Delta=M_{\Delta}-M_{N}$, to be of the order of $p$ that is taken to be of the order of $m_{\pi}$.

An additional new scale occurs for meson production in nucleon-nucleon collisions, namely the initial momentum $p_{i} \sim \sqrt{m_{\pi} M_{N}}$. Note that, although larger than the pion mass, this momentum is still smaller than the chiral symmetry breaking scale and thus the expansion should still converge, but slowly. A priori there are now two options to construct an effective field theory for pion production. The first option, called Weinberg scheme in what follows, treats all light scales to be of order of $m_{\pi}$. Thus, in this case, there is one expansion parameter, namely $\chi_{W}=m_{\pi} / M_{N}$. The other option is to expand in two scales simultaneously, namely $m_{\pi}$ and $p_{i}$. In this case the expansion parameter is

$$
\chi=\sqrt{\frac{m_{\pi}}{M_{N}}} \sim 0.4 .
$$

This scheme was advocated in Refs. [163,203] and applied in Ref. [204]. The two additional scales, namely $\Delta$ and $m_{\pi}$, are identified with

$$
\frac{\Delta}{\Lambda_{\chi}} \sim \frac{p_{i}}{\Lambda_{\chi}}=\chi \quad \text { and } \quad \frac{m_{\pi}}{\Lambda_{\chi}} \sim \frac{p_{i}^{2}}{\Lambda_{\chi}^{2}}=\chi^{2},
$$

where the former assignment was made due to the numerical similarity of the two numbers ${ }^{24}\left(\Delta=2.1 m_{\pi}\right.$ and $\left.p_{i}=2.6 m_{\pi}\right)$. Only explicit calculations can reveal which one is the more appropriate approach.

Within the Weinberg counting scheme, tree level calculations were performed for $s$-wave pion production in the reactions $p p \rightarrow p p \pi^{0}[162,163,173]$ as well as $p p \rightarrow p n \pi^{+}[164,165]$. In addition, complete calculations to next-to-next-toleading order (NNLO), where in the Weinberg scheme for the first time loops appear, are available for $p p \rightarrow p p \pi^{0}[205,206]$. The authors found that some of the NNLO contributions exceeded significantly the next-to-leading (NLO) terms leading them to the conclusion that the chiral expansion converges only

\footnotetext{
${ }^{24}$ Note that $\Delta$ stays finite in the chiral limit, whereas both $p_{i}$ as well as $m_{\pi}$ vanish.
} 
slowly, if at all. This point was further stressed in Refs. [207,155], however, it was shown recently that as soon as the scale induced by the initial momentum is taken into account properly (expansion in $\chi$ and not in $\chi_{W}$ ), the series indeed converges [203,204]. For illustration, in this section we compare the order assignment of the Weinberg scheme to that of the modified scheme.

In appendix E the relevant counting rules of the new scheme are presented as well as justified via application to a representative example. Especially, it is not clear a priori what scale to assign to the zeroth component $l_{0}$ of the four dimensional integration volume $d^{4} l$ as it occurs in covariant loops. After all, the typical energy of the system is given by $m_{\pi}$, but the momentum by $p_{i}$. As is shown in the appendix by matching a covariant analysis to one carried out in time-ordered perturbation theory, in loops $l_{0} \sim p_{i}$. This assignment was also confirmed in explicit calculations [204].

The starting point is an appropriate Lagrangian density, constructed to be consistent with the symmetries of the underlying more fundamental theory (in this case QCD) and ordered according to a particular counting scheme. Omitting terms that do not contribute to the order we will be considering here, we therefore have for the leading order Lagrangian [208,199,197]

$$
\begin{aligned}
\mathcal{L}^{(0)}= & \frac{1}{2} \partial_{\mu} \boldsymbol{\pi} \partial^{\mu} \boldsymbol{\pi}-\frac{1}{2} m_{\pi}^{2} \boldsymbol{\pi}^{2}+\frac{1}{2 f_{\pi}^{2}}\left[\left(\boldsymbol{\pi} \cdot \partial_{\mu} \boldsymbol{\pi}\right)^{2}-\frac{1}{4} m_{\pi}^{2}\left(\boldsymbol{\pi}^{2}\right)^{2}\right] \\
& +N^{\dagger}\left[i \partial_{0}-\frac{1}{4 f_{\pi}^{2}} \boldsymbol{\tau} \cdot(\boldsymbol{\pi} \times \dot{\boldsymbol{\pi}})\right] N \\
& +\frac{g_{A}}{2 f_{\pi}} N^{\dagger} \boldsymbol{\tau} \cdot \vec{\sigma} \cdot\left(\vec{\nabla} \boldsymbol{\pi}+\frac{1}{2 f_{\pi}^{2}} \boldsymbol{\pi}(\boldsymbol{\pi} \cdot \vec{\nabla} \boldsymbol{\pi})\right) N \\
& +\Psi_{\Delta}^{\dagger}\left[i \partial_{0}-\Delta\right] \Psi_{\Delta}+\frac{h_{A}}{2 f_{\pi}}\left[N^{\dagger}(\boldsymbol{T} \cdot \vec{S} \cdot \vec{\nabla} \boldsymbol{\pi}) \Psi_{\Delta}+\text { h.c. }\right]+\cdots
\end{aligned}
$$

The expressions for interactions with more than two pions depend on the interpolating field used. The choice made here was the so called sigma gaugec.f. Appendix A of Ref. [197], where also the corresponding vertex functions are given explicitly ${ }^{25}$.

For the next-to-leading order Lagrangian we get

${ }^{25}$ As usual, all observables are independent of the choice of the pion field. 


$$
\begin{aligned}
\mathcal{L}^{(1)}= & \frac{1}{2 m_{N}}\left[N^{\dagger} \vec{\nabla}^{2} N+\Psi_{\Delta}^{\dagger} \vec{\nabla}^{2} \Psi_{\Delta}\right] \\
& +\frac{1}{8 M_{N} f_{\pi}^{2}}\left(i N^{\dagger} \boldsymbol{\tau} \cdot(\boldsymbol{\pi} \times \vec{\nabla} \boldsymbol{\pi}) \cdot \vec{\nabla} N+h . c .\right) \\
& +\frac{1}{f_{\pi}^{2}} N^{\dagger}\left[\left(c_{2}+c_{3}-\frac{g_{A}^{2}}{8 m_{N}}\right) \dot{\boldsymbol{\pi}}^{2}-c_{3}(\vec{\nabla} \boldsymbol{\pi})^{2}-2 c_{1} m_{\pi}^{2} \boldsymbol{\pi}^{2}\right. \\
& \left.-\frac{1}{2}\left(c_{4}+\frac{1}{4 m_{N}}\right) \varepsilon_{i j k} \varepsilon_{a b c} \sigma_{k} \tau_{c} \partial_{i} \pi_{a} \partial_{j} \pi_{b}\right] N \\
& -\frac{g_{A}}{4 m_{N} f_{\pi}}\left[i N^{\dagger} \boldsymbol{\tau} \cdot \dot{\boldsymbol{\pi}} \vec{\sigma} \cdot \vec{\nabla} N+h . c .\right] \\
& -\frac{h_{A}}{2 m_{N} f_{\pi}}\left[i N^{\dagger} \boldsymbol{T} \cdot \dot{\boldsymbol{\pi}} \vec{S} \cdot \vec{\nabla} \Psi_{\Delta}+h . c .\right] \\
& -\frac{d_{1}}{f_{\pi}} N^{\dagger}(\boldsymbol{\tau} \cdot \vec{\sigma} \cdot \vec{\nabla} \boldsymbol{\pi}) N N^{\dagger} N \\
& -\frac{d_{2}}{2 f_{\pi}} \varepsilon_{i j k} \varepsilon_{a b c} \partial_{i} \pi_{a} N^{\dagger} \sigma_{j} \tau_{b} N N^{\dagger} \sigma_{k} \tau_{c} N+\cdots
\end{aligned}
$$

where $f_{\pi}$ denotes the pion decay constant in the chiral limit, $g_{A}$ is the axialvector coupling of the nucleon, $h_{A}$ is the $\Delta N \pi$ coupling, and $\vec{S}$ and $\boldsymbol{T}$ are the transition spin and isospin matrices, normalized such that

$$
\begin{aligned}
S_{i} S_{j}^{\dagger} & =\frac{1}{3}\left(2 \delta_{i j}-i \epsilon_{i j k} \sigma_{k}\right), \\
T_{i} T_{j}^{\dagger} & =\frac{1}{3}\left(2 \delta_{i j}-i \epsilon_{i j k} \tau_{k}\right) .
\end{aligned}
$$

These definitions are in line with the ones introduced in sec. 6.2.1. The dots symbolize that what is shown are only those terms that are relevant for the calculations presented. As demanded by the heavy baryon formalism, the baryon fields $N$ and $\Psi_{\Delta}$ are the velocity-projected pieces of the relativistic fields appearing in the interactions discussed in section 6.2.1; e.g. $N=1 / 2(1+\varphi) \psi$, where $v^{\mu}$ denotes the nucleon 4 -velocity.

The terms in the Lagrangians given are ordered according to the conventional counting $\left(p \simeq m_{\pi}\right)$. A reordering on the basis of the new scheme does not seem appropriate, for what order is to be assigned to the energies and momenta occurring depends on the topology of a particular diagram (see also Appendix E).

The constants $c_{i}$ can be extracted from a fit to elastic $\pi N$ scattering. This was 


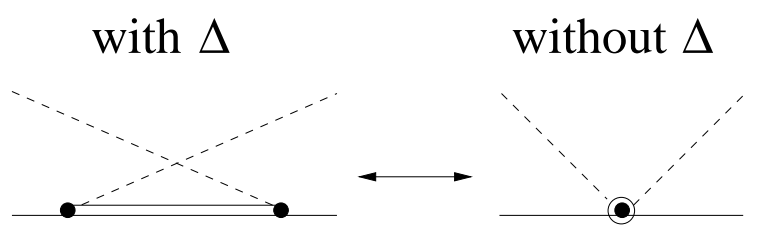

Fig. 25. Illustration of resonance saturation.

\section{Table 9}

The various low energy constants $c_{i}$ in units of $G e V^{-1}$. The first two columns give the values from the original references (left column: tree level calculation of Ref. [171]; right column: one loop calculation of Ref. [172]), whereas the last two columns give those values reduced by the Delta contribution as described in the text. It is those numbers that where used in the calculations presented here.

\begin{tabular}{|c|cc|cc|}
\hline$i$ & $c_{i}^{\text {tree }}$ & $c_{i}^{\text {loop }}$ & $c_{i}^{\text {tree }}(\Delta)$ & $c_{i}^{\text {loop }}(\Delta)$ \\
\hline 1 & -0.64 & -0.93 & -0.64 & -0.93 \\
2 & 1.78 & 3.34 & 0.92 & 0.64 \\
3 & -3.90 & -5.29 & -1.20 & -2.59 \\
4 & 2.25 & 3.63 & 0.9 & 2.28 \\
\hline
\end{tabular}

done in a series of papers with successively improved methods [171,172,209212]. However, here we will focus on the values extracted in Refs. [171,172] for it is those that were used in the calculations for pion production in nucleonnucleon collisions. In the former work the $c_{i}$ were extracted at tree level and in the latter to one loop. The corresponding values are given in table 9 . In both papers the Delta isobar was not considered as explicit degree of freedom. In an effective field theory the low energy constants appearing depend on the dynamical content of the theory. Thus, in a theory without explicit Deltas, their effect is absorbed in the low energy constants [172]. This is illustrated graphically in Fig. 25. Thus we need to subtract the Delta contribution from the values given in the first columns of table 9 . Analytical results for those contributions are given in Refs. $[213,172]^{26}$ :

$$
c_{2}^{\Delta}=-c_{3}^{\Delta}=2 c_{4}^{\Delta}=\frac{h_{A}^{2}}{9\left(M_{\Delta}-M_{N}\right)}=2.7 \mathrm{GeV}^{-1}
$$

\footnotetext{
${ }^{26}$ To match the results of the two references the large $N_{C}$ value has to be used for $h_{A}=3 g_{A} / \sqrt{2} \simeq 2.7$.
} 


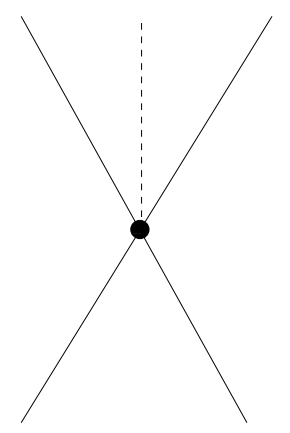

a)

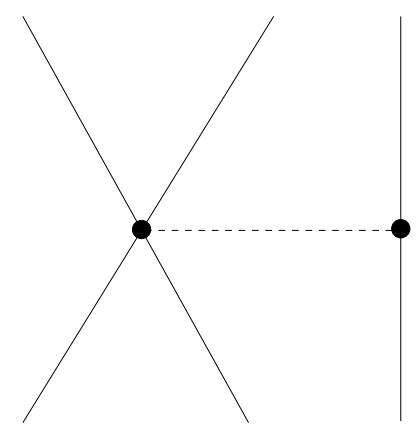

b)

Fig. 26. Illustration of the role of the $4 N \pi$ contact term in $N N \rightarrow N N \pi$ and three nucleon scattering. Solid lines denote nucleons, dashed lines denote pions.

Thus, the only undetermined parameters in the interaction Lagrangian are $d_{1}$ and $d_{2}$. Since they are the strength parameters of 4-nucleon contact interactions that do not contain any derivatives of the nucleon fields, they only contribute to those amplitudes that have an $N N S$-wave to $N N S$-wave plus pion $p$-wave transition. This automatically excludes a transition from an isospin triplet to an isospin triplet state, for this would demand to go from ${ }^{1} S_{0}$ to ${ }^{1} S_{0}$ accompanied by a $p$-wave pion, which is forbidden by conservation of total angular momentum. Thus, only two transitions are possible: $T=0 \rightarrow T=1$, as it can be studied in $p n \rightarrow p p \pi^{-}$, and $T=1 \rightarrow T=0$, as it can be studied in $p p \rightarrow p n \pi^{+}$. Even more importantly, in both channels the $d_{i}$ appear with the same linear combination $d$ which is completely fixed by the corresponding isospin factors:

$$
d=\frac{1}{3}\left(d_{1}+2 d_{2}\right) \sim \frac{\delta}{f_{\pi}^{2} M_{N}},
$$

where we have introduced the dimensionless parameter $\delta$. In order for the counting scheme to work, $\delta=\mathcal{O}(1)$ has to hold. As we will show below, this order of magnitude is indeed consistent with the data. Please note, the fournucleon-pion contact term with $p$-wave discussed here was to some extend also investigated in Ref. [214], however, within a different expansion scheme. Thus direct comparison is not possible.

The parameter $d$ is very interesting, for it is at the same time the leading shortrange-long-range contribution ${ }^{27}$ to the three nucleon force, as illustrated in $\overline{{ }^{27} \text { In this }}$ context, pion exchanges are called long-ranged, whereas any exchange of heavier mesons - absorbed in the contact terms - are called short-ranged. 
Fig. 26. Naturally, it can also be fixed from $p d$ scattering data directly. This was done in Ref. [215]. We come back to this point below.

The effect of the large scale on the vertices is best illustrated with an example. In $\mathcal{L}^{(0)}$ the so-called Weinberg-Tomozawa (WT) term $1 /\left(4 f_{\pi}^{2}\right) N^{\dagger} \boldsymbol{\tau} \cdot(\boldsymbol{\pi} \times \dot{\boldsymbol{\pi}}) N$ appears. The corresponding recoil correction $1 /\left(8 M_{N} f_{\pi}^{2}\right)\left(i N^{\dagger} \boldsymbol{\tau} \cdot(\boldsymbol{\pi} \times \vec{\nabla} \boldsymbol{\pi})\right.$. $\vec{\nabla} N+$ h.c.) appears in $\mathcal{L}^{(1)}$. Therefore the vertex function derived from the WT term is proportional to $\left(q_{0}+k_{0}\right) / f_{\pi}^{2}$, where $q_{0}\left(k_{0}\right)$ denote the zero-th component of the 4 -vectors for the outgoing (incoming) pion. If the WT term appears as the $\pi N$ vertex in the rescattering contribution (c.f. Fig. 2b), in threshold kinematics $k_{0}=m_{\pi} / 2$ and $q_{0}=m_{\pi}$. Also in threshold kinematics, where the 3-momentum transfer equals the initial momentum, we find for the contribution from the recoil term $p^{2} /\left(2 M_{N} f_{\pi}^{2}\right)$. Obviously, since $p^{2}=M_{N} m_{\pi}$, the contribution from the WT term and from its recoil term are of the same order. This changes if the WT term appears inside a loop, for then the scale for $k_{0}$ is also set by $p$-in this case the recoil term is suppressed by one chiral order compared to the WT term itself. The assignments made are confirmed by explicit calculation [204] as well as by a toy model study [174].

Since we know now the interactions of pions and nucleons we can investigate the relevance of subleading loops, where we mean loops that can be constructed from a low order diagram by inserting an additional pion line. Obviously this procedure introduces at least one $\pi N N$ vertex $\sim p / f_{\pi}$, a pion propagator $\sim 1 / p^{2}$, an integral measure $p^{4} /(4 \pi)^{2}$ and either an additional $\pi N N$ vertex together with two nucleon propagators, or one additional nucleon propagator together with a factor $1 / f_{\pi}$. To get the leading piece of the loops in the latter case, the integration over the energy variable has to pick the large scale and thus we are to count the nucleon propagator as $1 / p$, leading to an overall suppression factor for this additional loop of $p^{2} / M_{N}^{2}$. In the former case, however, a topology is possible that contains a two-nucleon cut. This unitarity cut leads to an enhancement of that intermediate state, for it pulls a large scale $\left(M_{N}\right)$ into the numerator. Based on this observation Weinberg established a counting scheme for $N N$ scattering that strongly differs from that in $\pi \pi$ as well as $\pi N$ scattering [186]. The corresponding factor introduced by this loop is $p / M_{N}$ (and in addition typically comes with a factor of $\pi$ ). As was stressed 
Table 10

Comparison of the corresponding chiral order in the Weinberg scheme $\left(p \sim m_{\pi}\right.$ ) and the new counting scheme ( $p \sim \sqrt{m_{\pi} M_{N}}$ ) for several nucleonic contributions for $p$-wave pion production. Subleading vertices are marked as $\odot$. Here $q$ denotes the external pion momentum. For simplicity we assume the outgoing nucleons at rest.

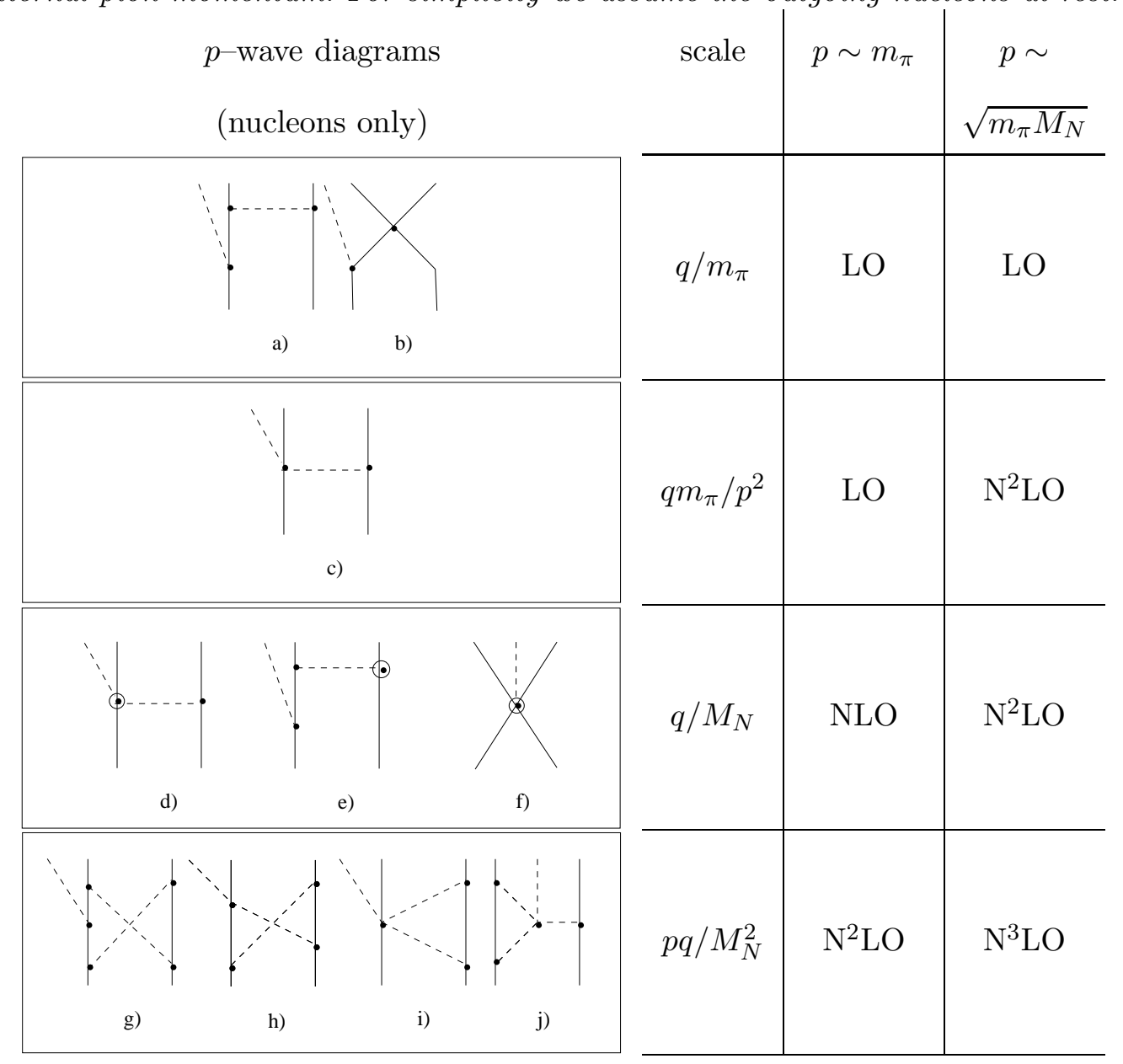

by Weinberg, this suppression is compensated by the size of the corresponding low energy constants of the two-nucleon interaction and therefore all diagrams that can be cut by crossing a two nucleon line only are called reducible and the initial as well as final state $N N$ interaction is summed to all orders. This is what is called distorted wave Born approximation. There is one exception to this rule: namely when looking at the two-nucleon intermediate state close to the pion production vertex in diagram a of Fig. 2. Either the incoming or the outgoing nucleon needs to be off-shell and thus this intermediate state does not allow for a two nucleon unitarity cut. Therefore those two-nucleon intermediate states are classified as irreducible. 
There is a special class of pion contributions not yet discussed, namely those that contain radiative pions (on-shell pions in intermediate states). We restrict ourselves to a kinematic regime close to the production threshold. As soon as an intermediate pion goes on-shell, the typical momentum in the corresponding loop automatically needs to be of order of the outgoing momenta. This leads to an effective suppression of radiative pions; e.g., for $s$-wave pions the effects of pion retardation become relevant at $\mathrm{N}^{5} \mathrm{LO}$.

Note that each loop necessarily contributes at many orders simultaneously. The reason for this is that the two scales inherent to the pion production problem can be combined to a dimensionless number smaller than one: $m_{\pi} / p_{i}=\chi$ . However, what can always be done on very general grounds is to assign the minimal order at which a diagram can start to contribute and this is sufficient for an efficient use of the effective field theory.

The next step is the consistent inclusion of the nuclear wave functions. However, the $N N$ potentials constructed consistently with chiral perturbation theory [198-201] are not applicable at the pion production threshold. Therefore we use the so-called hybrid approach originally introduced by Weinberg [216], where we convolute the production operator, constructed within chiral perturbation theory, with a phenomenological $N N-N \Delta$ wavefunction. We use the CCF model described in the previous section [175].

Let us start with a closer look at the production of $p$-wave pions, for those turned out easier to handle than $s$-wave pions. The reason for this pattern lies in the nature of pions as Goldstone bosons of the chiral symmetry: since in the chiral limit for vanishing momenta the interaction of pions with matter has to vanish, the coupling of pions naturally occurs in the company of either a derivative or an even power of the pion mass ${ }^{28}$. As a consequence, the leading piece of the $\pi N N$ vertex is of $p$-wave type, whereas the corresponding $s^{-}$ wave piece is suppressed as $\omega_{\pi} / M_{N}$, where $\omega_{\pi}$ denotes the pion energy. Note

\footnotetext{
$\overline{28}$ Only even powers of the pion mass are allowed to occur in the interaction, since, due to the Gell-Mann-Oakes-Renner relation [217], $m_{\pi}^{2} \propto m_{q}$, where $m_{q}$ is the current quark mass and in the interaction no terms non-analytic in the quark masses are allowed.
} 


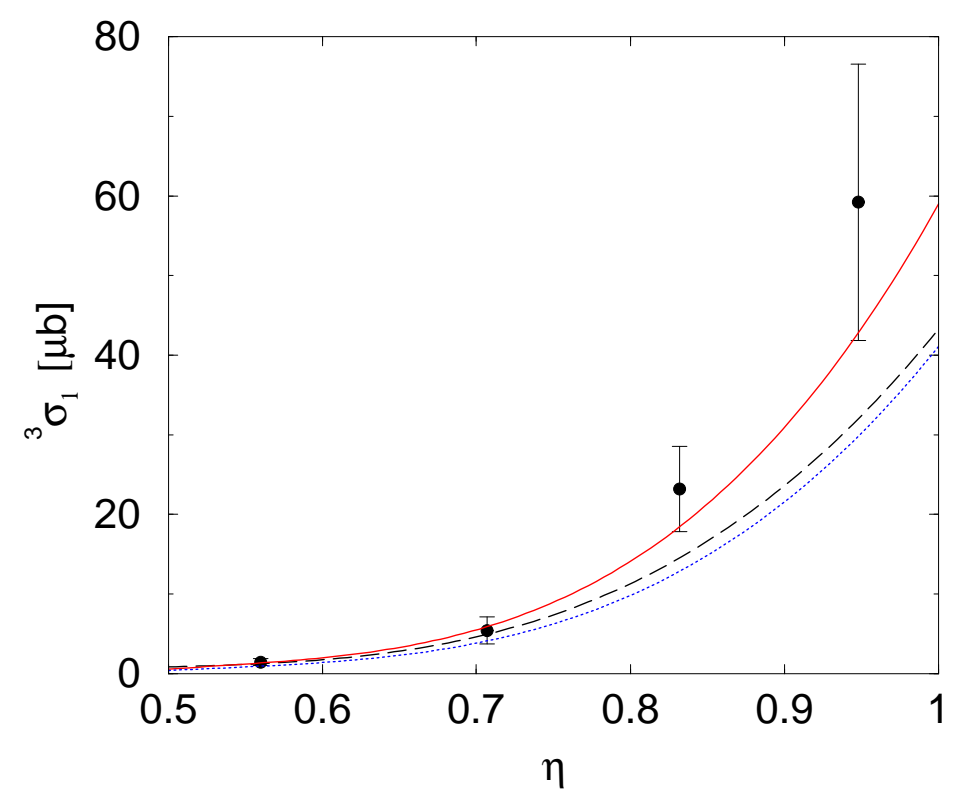

Fig. 27. Comparison of the predictions from effective field theory at LO (dashed line) and $N L O$ (with the $\pi N$ parameters from a NLO (dotted line) and an NNLO (solid line) analysis) with the ${ }^{3} \sigma_{1}$ cross section for $p p \rightarrow p p \pi^{0} \quad$ [47].

that also in the case of neutral pion photoproduction the $s$-wave amplitude is dominated by pion loops, whereas the $p$-wave amplitude is dominated by tree level diagrams $[218,197]$.

The corresponding diagrams are shown in table 10 up to $\mathrm{N}^{3} \mathrm{LO}$. So far in the literature calculations have been carried out only up to $\mathrm{N}^{2} \mathrm{LO}$ [203]. An important test of the approach is to show its convergence. For that we need an observable to which $s^{-}$wave pions do not contribute. Such an observable is given by the spin cross section ${ }^{3} \sigma_{1}$ recently measured at IUCF for the reaction $p p \rightarrow p p \pi^{0}$ [47] (c.f. table 7 in section 4.2). The parameter-free prediction of chiral perturbation theory compared to the data is shown in Fig. 27. As one can see, the total amplitude is clearly dominated by the leading order suggesting a convenient rate of convergence for the series. In addition, the prediction agrees with the data.

Thus we are now prepared to extract the parameter $d$ from data on the reaction $p p \rightarrow p n \pi^{+}$[219]. As it was argued above, only the amplitude corresponding to the transition ${ }^{1} S_{0} \rightarrow{ }^{3} S_{1} p$, called $a_{0}$, is influenced by the corresponding contact interactions. The results of the chiral perturbation theory calculations 


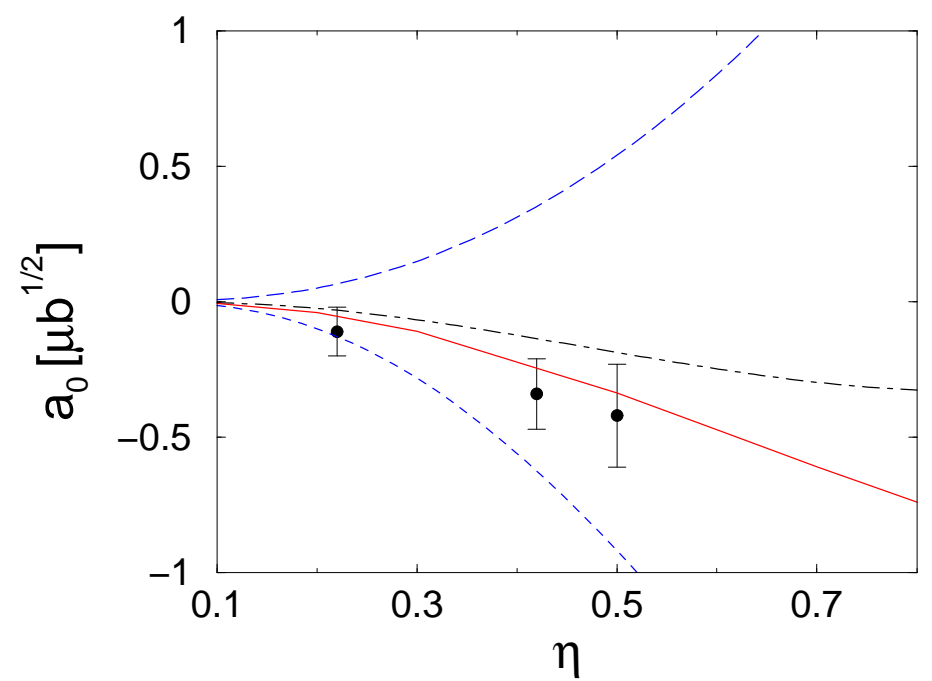

Fig. 28. $a_{0}$ of $p p \rightarrow n p \pi^{+}$in chiral perturbation theory. The different lines correspond to values of the parameter related to the three-nucleon force: $\delta=1$ (long dashed line). $\delta=0$ (dot-dashed line), $\delta=-0.2$ (solid line), and $\delta=-1$ (short dashed line). Data are from Ref. [219].

are shown in Fig. 28. The figure shows four curves for different values of the parameter $\delta$ defined in Eq. (86), namely the result for $\delta=0$ (dot-dashed line), for $\delta=-0.2$ - the authors of Ref. [220] claim this value to yield an important contribution to $A_{y}$ in $N d$ scattering at energies of a few $\mathrm{MeV}^{29}$ - as well as the results we get when $\delta$ is varied within its natural range $\delta=+1$ and $\delta=-1$ shown as the long-dashed and the short-dashed curve, respectively. Thus we find that the results for $a_{0}$ are indeed rather sensitive to the strength of the contact interaction. This might be surprising at first glance, since we are talking about a subleading operator, however it turned out that the leading (diagrams a) and b) in table 10) and subleading contributions (the same diagrams with a $\Delta$ intermediate state) largely cancel. Thus, to draw solid conclusions the $p$-wave calculations should be improved by one chiral order, the corresponding diagrams of which are shown in table $10 \mathrm{~g})-\mathrm{j}$ ).

Please note that the rescattering contribution involving $c_{4}$ that occurs at the same order as the $d$ contribution turned out to be sensitive to the regulator used for the evaluation of the convolution integral with the nuclear wavefunction. This cutoff dependence can be absorbed in $d$, which in turn is now cutoff-dependent. Therefore, in order to compare the results for $d$ from the pion production reaction to those extracted from the three-nucleon system

${ }^{29}$ The calculation of Ref. [220] suffers from numerical problems. 
[215] a consistent calculation that is not possible at present has to be performed. Note that the $d$ parameter as fixed in Ref. [215] (there it is called E) also turned out to be sensitive to the regulator. On the long run, however, a consistent description of pion production and three-nucleon scattering should be a rather stringent test of chiral effective field theories at low and intermediate energies. As should be clear from the discussion above, to yield values for the low energy constant $\delta$ that are compatible both calculations $p d$ scattering as well as $\pi$-production have to be performed using the same dynamical fields - at present the $\Delta$-isobar is not considered as explicit degree of freedom in Ref. [215], but plays a numerically important role in the extraction of $\delta$ from $N N \rightarrow N N \pi$.

In section 4.3 it was shown that the differential cross section as well as the analysing power for the reaction $p n \rightarrow p p \pi^{-}$is sensitive to an interference term of the $s^{-}$-wave pion production amplitude of the $A_{11}$ amplitude $\left({ }^{3} P_{0} \rightarrow{ }^{1}\right.$ $S_{0} s$ ) and the $p$ amplitudes of $A_{01}:{ }^{3} S_{1} \rightarrow{ }^{1} S_{0} p$ and ${ }^{3} D_{1} \rightarrow{ }^{1} S_{0} p$. Obviously, the 4-nucleon contact interaction contributes to the former. Thus, once a proper chiral perturbation theory calculation is available for the $s$-wave pion production - whose status will be discussed in the subsequent paragraphsthe reaction $p n \rightarrow p p \pi^{-}$close to the production threshold might well be the most sensitive reaction to extract the parameter $d$.

Let us now turn to the $s$-wave contributions. The leading diagrams containing nucleons only are shown in table 11 ; those that contain the $\Delta$ are shown in table 12. Again, the Weinberg scheme and the new scheme are compared. The list is complete up to NNLO in both schemes. Please note, however, that one class of diagrams ( $k$ and $l$ ) that is of NLO in the Weinberg counting in the new scheme is pushed to $\mathrm{N}^{4} \mathrm{LO}$ !

Thus in the new counting scheme - contrary to the Weinberg scheme - the leading pieces of some loops appear one order lower than the tree level isoscalar rescattering amplitudes. As can also be read from the table, the corresponding order is $m_{\pi} / M_{N}$. If we consider, in addition, that due to the odd parity of the pion the initial state has to be a $p$-wave, the loops themselves have to scale as $\sqrt{m_{\pi}}$. Since no counter term non-analytic in $m_{\pi}$ is allowed, the loops have 
Table 11

Comparison of the corresponding chiral order in the Weinberg scheme $\left(p \sim m_{\pi}\right.$ ) and the new counting scheme ( $p \sim \sqrt{m_{\pi} M_{N}}$ ) for several nucleonic contributions for $s$-wave pion production. Subleading vertices are marked as $\odot$. Not shown explicitly are the recoil corrections for low-order diagrams. For example, recoil corrections to diagram b) appear at order $\mathrm{pm}_{\pi} / M_{N}^{2}$.

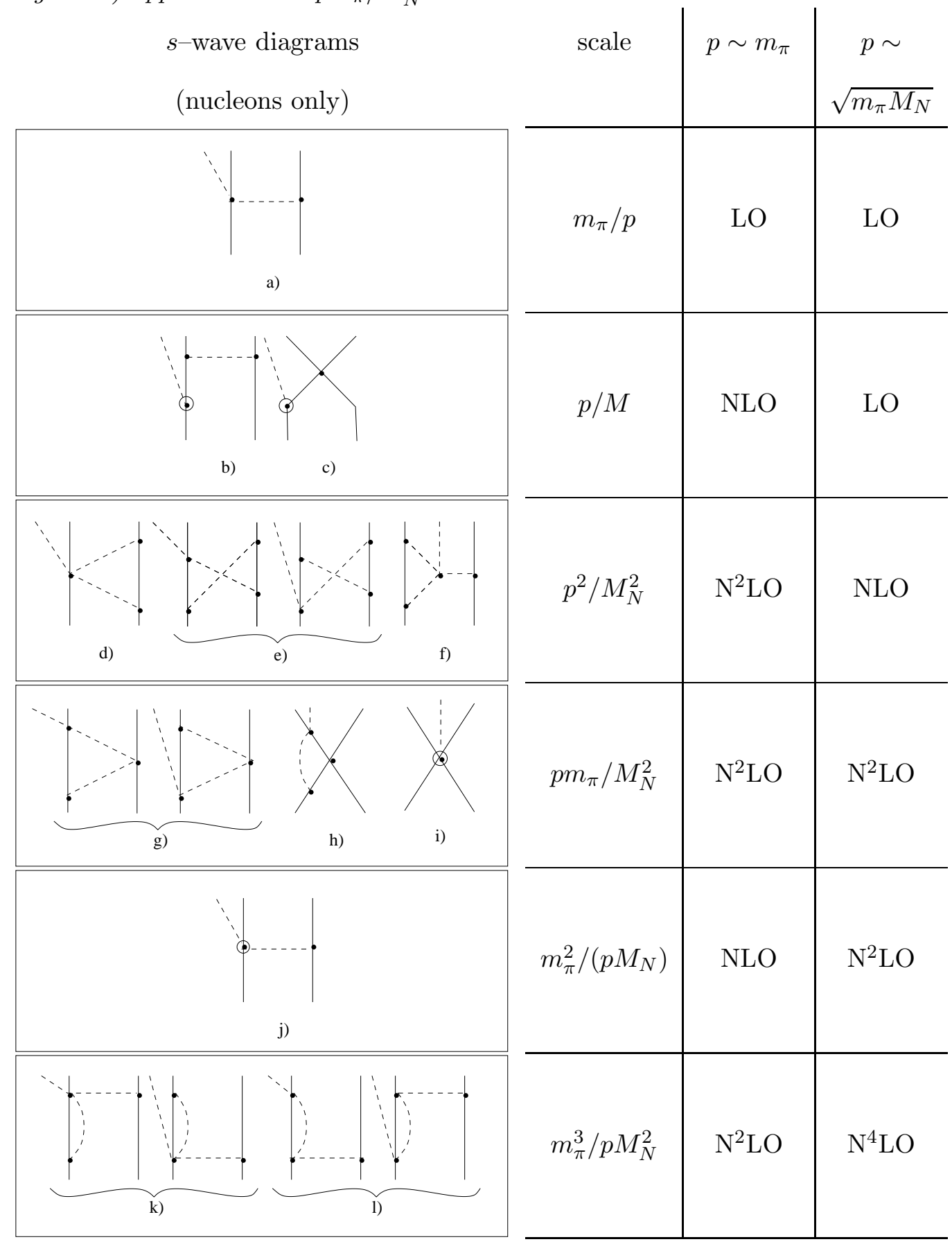


Table 12

Comparison of the corresponding chiral order in the Weinberg scheme $\left(p \sim m_{\pi}\right.$ ) and the new counting scheme $\left(p \sim \sqrt{m_{\pi} M_{N}}\right)$ for the leading and next-to-leading $\Delta$ contributions for s-wave pion production. Subleading vertices are marked as $\odot$. Not shown explicitly are the recoil corrections for low-order diagrams.

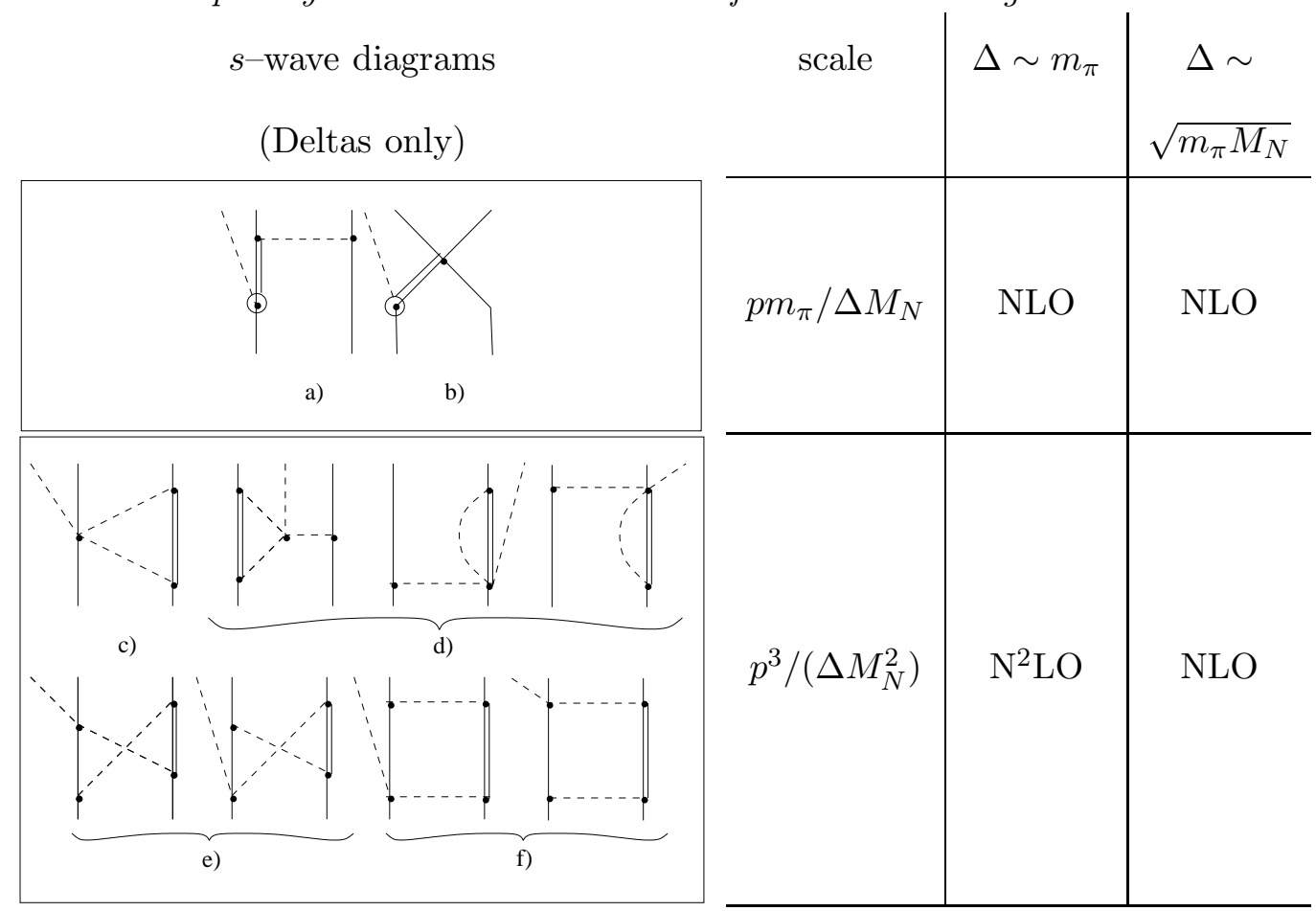

to be finite or to cancel exactly. This requirement is an important consistency check of the new counting scheme.

The details of the loops calculations in threshold kinematics can be found in Ref. [204]. Here we only give the results. Note, we only evaluate the leading order pieces of the integrals corresponding to the diagrams of table (11). For example, in the integrals we drop terms of order $m_{\pi}$ compared to $l_{0}$.

After these simplifications, straightforward evaluation gives for the production amplitude from the loops with nucleons only

$$
A_{n}=\frac{i}{F_{\pi}^{3}} g_{A}^{3}\left(\vec{\sigma}_{1} \cdot \frac{\vec{k}}{|\vec{k}|}\right)\left(\frac{M_{N} m_{\pi}}{128 F_{\pi}^{2}}\right) \iota_{n},
$$

where $n$ denotes the diagram (labels as in the figure). For the isospin functions we find 


$$
\iota_{d}=-\tau_{1}^{c}, \quad \iota_{e}=-\frac{1}{4}\left(\tau_{1}^{c}+\tau_{2}^{c}\right), \quad \iota_{f}=\frac{3}{2} \tau_{1}^{c}
$$

Those can be easily evaluated in the different isospin channels. We find for $\iota\left(T T_{3}\right)=\left\langle T T_{3}\left|\iota_{d}+\iota_{e}+\iota_{f}\right| 11\right\rangle$ (it is sufficient here to look at the $p p$ initial state only)

$$
\iota(11)=-1-\frac{1}{2}+\frac{3}{2}=0, \quad \iota(00)=-1+0+\frac{3}{2}=-\frac{1}{2}
$$

The first observation is that the NLO contributions are of the order of magnitude expected by the power counting, since

$$
\left(\frac{M_{N} m_{\pi}}{128 F_{\pi}^{2}}\right)=0.8 \chi^{2}
$$

where we used $F_{\pi}=93 \mathrm{MeV}$. The power counting proposed in Refs. [163,203] thus is indeed capable of treating properly the large scale inherent to the the $N N \rightarrow N N \pi$ reaction.

We checked that our results for the individual diagrams agree with the leading non-vanishing pieces from the calculations of Ref. [205] ${ }^{30}$. In addition, for almost all diagrams given in table 11, Ref. [205] gives explicit numbers for the amplitudes in threshold kinematics. It is intriguing to compare those to what one expects from the different counting schemes. This is done in table 13, where the first line specifies the particular diagram according to table 11 and the second gives the result of the analytical calculation of Ref. [205] ${ }^{31}$ (normalized to the first column). In the following two lines those numbers are compared to the expectations based on the counting schemes - first showing those for the Weinberg scheme and then those for the new counting scheme. As can clearly be seen, the latter does an impressive job of predicting properly the hierarchy of diagrams. Thus, at least when ISI and FSI are neglected, the

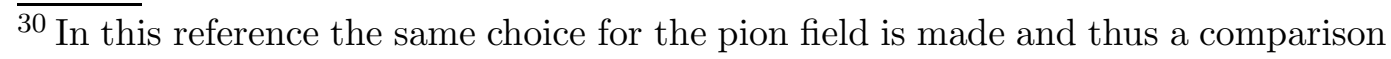
of individual diagrams makes sense. Note that there is a sign error in the formula for diagram $f$ in Ref. [205]. We are grateful to F. Myhrer for helping us to resolve this discrepancy.

${ }^{31}$ In Ref. [205] the full result for the particular loops are given. Thus, any loop contains higher order contributions. 
Table 13

Comparison of the results of the analytic calculation of Ref. [205] with the expectations based on the two counting schemes as discussed in the text. The diagrams are labeled as in Fig. 11 (the label $j_{R}$ shows that here the recoil term of diagram $j$ is calculated; it appears at NNLO in the Weinberg scheme as well as in the new counting scheme).

\begin{tabular}{c|c|c|c|c|c|c|} 
Diagram & $\mathrm{d}$ & $\mathrm{e}$ & $\mathrm{g}$ & $j_{R}$ & $\mathrm{k}$ & $\mathrm{l}$ \\
\hline Ref. $[205]$ & 1.0 & -1.0 & 0.1 & 0.4 & 0.03 & 0.02 \\
$p \sim m_{\pi}$ & 1.0 & 1.0 & 1.0 & 1.0 & 1.0 & 1.0 \\
$p \sim \sqrt{m_{\pi} M_{N}}$ & 1.0 & 1.0 & 0.4 & 0.4 & 0.06 & 0.06
\end{tabular}

counting scheme proposed is capable of dealing with these large momentum transfer reactions.

It is striking that the sum of loops in the case of the neutral pion production vanishes $(\iota(11)=0$ in Eq. (89)). In addition, for neutral pion production there is no meson exchange current at leading order and the nucleonic current (diagrams b) and c) in table 11) gets suppressed by the poor overlap of the initial and final state wave functions (see discussion in sec. 1.4) - an effect not captured by the counting - and interferes destructively with the direct production off the Delta (diagrams a) and b) in table 12). Thus the first significant contributions to the neutral pion production appear at NNLO. This is the reason why many authors found many different mechanisms, all of similar importance and capable of removing the discrepancy between the Koltun and Reitan result and the data, simply because there is a large number of diagrams at NNLO. The situation is very different for the charged pions. Here there is a meson exchange current at leading order and there are nonvanishing loop contributions. We therefore expect charged pion production to be significantly better under control than neutral pion production and this is indeed what we found in the phenomenological model described in the previous section.

Next let us have a look at the loops that contain Delta isobars (c.f. Fig. 12). In Ref. [204] it was shown that the individual loops diverge already at leading order, because the Delta-Nucleon mass difference introduces a new 
scale. Therefore, as was argued above, the sum of the diagrams has to cancel, for at NLO there is no counter term. Is is an important check of the counting scheme that this does indeed happen. We take this as a strong indication that expanding in $\chi$ is consistent with the chiral expansion.

The observation that we have now established a counting scheme for reactions of the type $N N \rightarrow N N \pi$ also has implications for the understanding of other reactions. One example is the analysis elastic $\pi d$ scattering, which is commonly used to extract the isoscalar $\pi N$ scattering length that is difficult to get at otherwise [167]. As mentioned above, due to chiral symmetry constraints the isoscalar scattering length does not get a contribution at leading order and thus, for an accurate extraction of this important quantity from data on $\pi d$ scattering or on $\pi d$ bound states, a controlled calculation of the few bodycorrections is compulsory. One of these corrections are the so-called dispersive corrections (see Ref. [221] and references therein): loop contributions to the elastic scattering that have intermediate two-nucleon states. Obviously, the imaginary part of those loops gives the essential contribution to the imaginary part of the $\pi d$ scattering length ${ }^{32}$, however there is also a real part to these loops that needs to be calculated within a scheme consistent with that used for the calculation of the other contributions. Within chiral perturbation theory that has not been done up to now. Given the progress reported here, such a calculation is now feasible. The same technique can then also be used to calculate the corresponding corrections for $\pi^{3} \mathrm{He}$ scattering, recently calculated in chiral perturbation theory for the first time [223].

\subsection{On the significance of off-shell effects}

A few years a ago there existed a strong program at several hadron facilities to measure bremsstrahlung in $N N$ collisions with the goal to identify the true off-shell behavior of the $N N$ interaction. Indeed it was found that the predictions for several highly differential observables are significantly different when different $N N$ potentials are employed.

\footnotetext{
${ }^{32}$ About one third of the imaginary part was found to be related to the reaction $\pi d \rightarrow \gamma N N[222]$.
} 


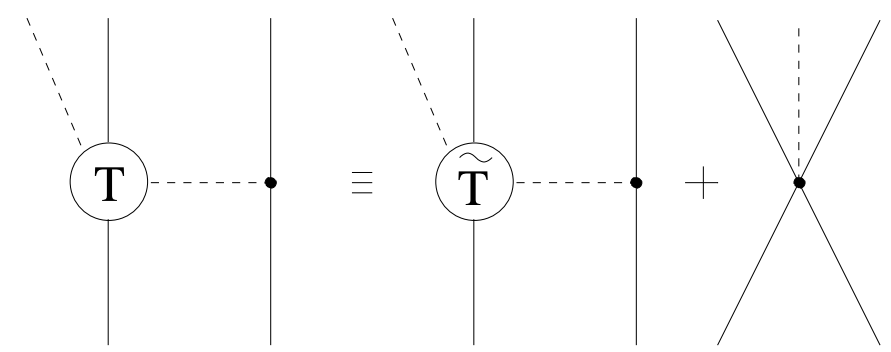

Fig. 29. Visualisation of Eq. (90).

On the other hand, a change in the off-shell behavior of any $T$-matrix can be realized on the level of the effective interaction by a field redefinition and it is known since long that $S$ matrix elements do not change under those transformations (as long as one works within a well defined field theory)[224,225]. Thus, for any given set of fields the off-shell amplitudes might well have a significant impact on the values of various observables, but it is an intrinsic feature of quantum field theory that they can not be separated from the short range interactions constructed within the same model space. In a very pedagogical way those results were presented later in Refs. [226,227].

How can one understand this seemingly contradictory situation: on the one hand off-shell amplitudes enter the evaluation of matrix elements and in some cases influence significantly the result (c.f. discussion in previous sections), on the other hand they are claimed not to have any physical significance? Following Ref. [226] to start the discussion, let us consider some general halfoff shell $\pi N T$-matrix. In a covariant form the list of its arguments contains the standard Mandelstam variables $s, t$ as well as $q^{2}$ - the four momentum squared of the off-shell particle(s). For our discussion let this be the incoming pion and for simplicity omit all spin indices. We now define $T_{R}\left(s, t, q^{2}\right)$ via

$$
T\left(s, t, q^{2}\right)=\tilde{T}\left(s, t, q^{2}\right)+\left(q^{2}-m_{\pi}^{2}\right) T_{R}\left(s, t, q^{2}\right)
$$

where $\tilde{T}\left(s, t, q^{2}\right)$ is arbitrary up to the condition that it has to agree with the on-shell amplitude for $q^{2}=m_{\pi}^{2}$. Obviously, this is always possible and we can assume $T_{R}$ to be smooth around the on-shell point. Then, when introduced into the $N N \rightarrow N N \pi$ transition amplitude, we get

$$
A=T \frac{1}{q^{2}-m_{\pi}^{2}} W_{\pi N N}=\tilde{T} \frac{1}{q^{2}-m_{\pi}^{2}} W_{\pi N N}+T_{R} W_{\pi N N}
$$

where $W_{\pi N N}$ denotes the $\pi N N$ vertex function. This example highlights two 
important facts: i) a change in the off-shell dependence of a particular amplitude can always be compensated via an appropriate additional contact term. The only quantities that are physically accessible are $S$-matrix elements and those are by definition on-shell; and ii) the contribution stemming from the off-shell $\pi N T$-matrix is a short range contribution. Therefore the distinction between short range and off-shell rescattering is artificial.

\subsection{Lessons and outlook}

The previous section especially made clear that it is rather difficult to construct a model that gives quantitatively satisfactory results for the reaction $p p \rightarrow p p \pi^{0}$. On the other hand, it turned out that for the reaction $p p \rightarrow p n \pi^{+}$ current models as well as the effective field theory approach do well. In the previous sections this difference was traced back to a suppression of meson exchange currents in the neutral pion production. What is the lesson to be learned from this? First of all, any model for meson production close to the threshold should contain the most prominent meson exchange currents; as long as these are not too strongly suppressed, one can expect them to dominate the total production cross section close to the threshold. However if there is no dominant meson exchange current, then there is a large number of sub-leading operators that compete with each other and make a quantitative understanding of the cross section difficult. The optimistic conclusion to be drawn from these observations is that (also for heavier mesons) the leading meson exchange currents should give a reasonable description of the data, while the contributions from irreducible loops largely cancel. Obviously, in heavy meson production there is no reason anymore to consider pions only as the exchange particles. In this sense it is $\pi^{+}$production that is the typical case, whereas the $\pi^{0}$ is exceptional due to the particular constraints from chiral symmetry in that channel. Note that also in the case of pion photoproduction close to the threshold the $\pi^{0}$ plays a special role, in that the $s$-wave amplitude is dominated by loops [218].

Let us look in somewhat more detail at the production operator for neutral pion production. Within the model described above, the most prominent di- 


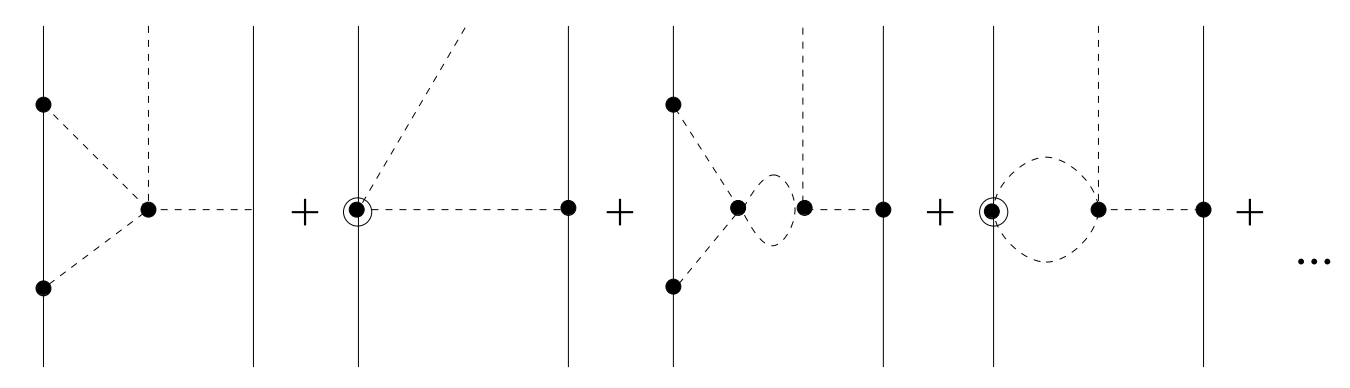

Fig. 30. The one sigma exchange as it is perturbatively built up in the effective field theory, starting from the left with the lowest order diagram (NLO). The chiral order increases by one power in $\chi$ between each diagram from the left to the right.

agram for neutral pion production close to the production threshold is pion rescattering via the isoscalar pion-nucleon $T$-matrix that, for the kinematics given, is dominated by a one-sigma exchange (diagram b) of Fig. 2, where the $T$-matrix is replaced by the isoscalar potential given by diagram e) of Fig. 22). Within the effective field theory the isoscalar potential is built up perturbatively. This is illustrated in Fig. 30. As was shown above, the leading piece of the one-sigma exchange gets canceled by other loops that cannot be interpreted as a rescattering diagram (the sum of diagrams d), e) and f) of table 11 vanishes) and are therefore not included in the phenomenological approach. This is an indication that in order to improve the phenomenological approach, at least in case of neutral pion production, pion loops should be considered as well ${ }^{33}$.

There is one more important conclusion to be drawn from the insights reported in the two previous sections: We can now identify what was missing in the straightforward extension of the Bonn potential reported in Ref. [24]. This is especially relevant, since we will see that the failure to describe the low energy pion production data in that approach does not point at a missing degree of freedom in the nucleon-nucleon phenomenology, but at an incomplete treatment of the cut structure.

Diagrammatically, the dressed $\pi N N$ vertex function is given by

\footnotetext{
$\overline{33}$ Note that within the effective field theory approach the convergence of the series shown in Fig. 30 should also be checked, as pointed out in a different context in Ref. [228].
} 


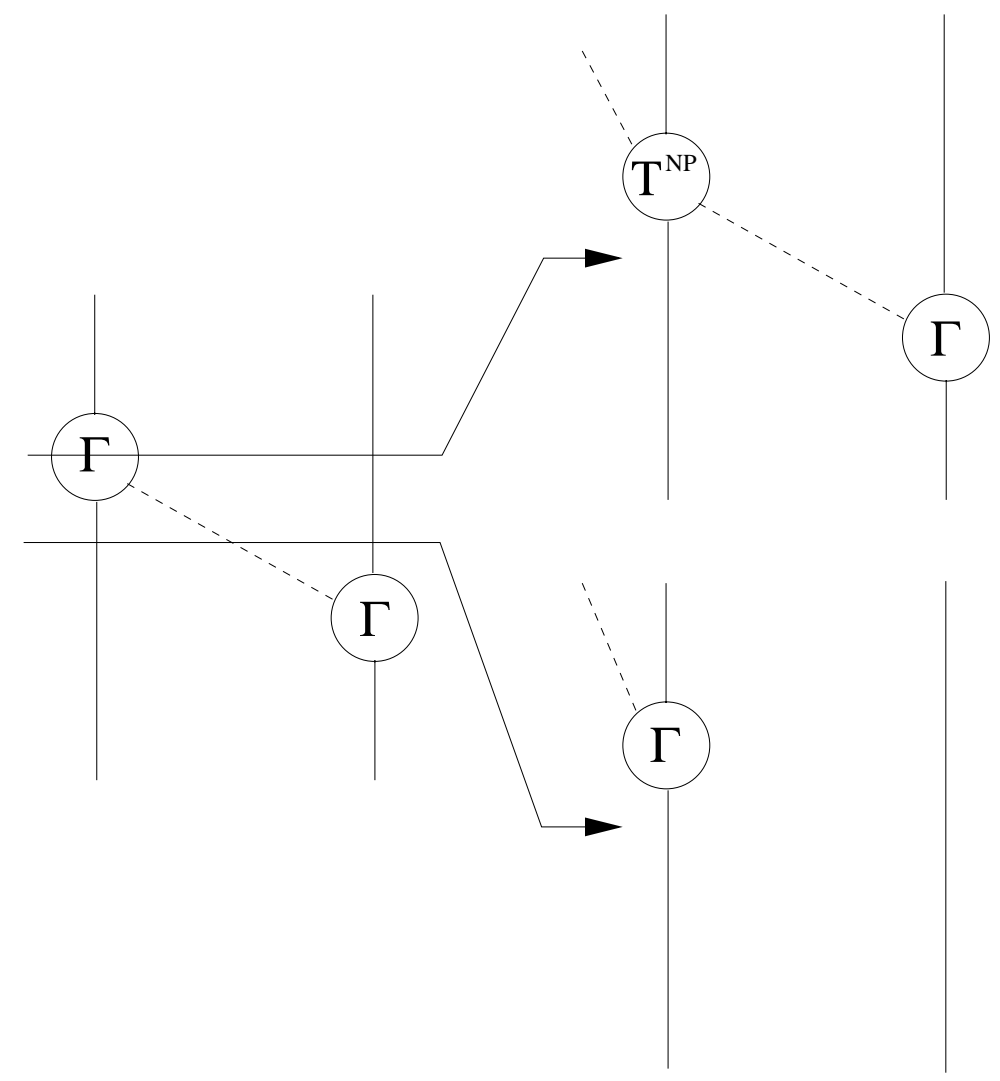

Fig. 31. Leading cut structure of the one pion exchange potential in nucleon-nucleon scattering.

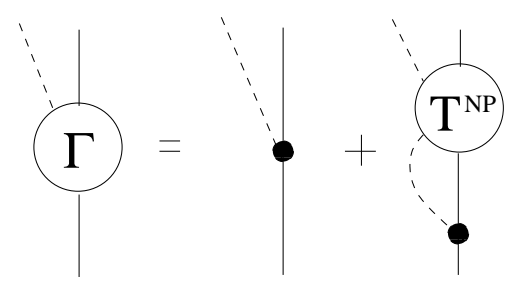

where the solid dot indicates the so-called bare vertex (in this picture it is to be understood as a parameterization of both the extended structure of the nucleon as well as meson dynamics not included in the non-pole $\pi N T$-matrix $T^{N P}$, such as $\pi \rho$ [229] or $\pi \sigma$ [230] correlations) as well as the dressing due to the $\pi N$ interactions parameterized in $T^{N P}$. The latter structure, however, contains a $\pi N$ cut not considered in Ref. [24]. It is straightforward to map the different three-body cuts in the one pion exchange potential to those that must occur in the pion production operator in order to allow for a consistent description of scattering and production. This is indicated in Fig. 31: the additional cut due to the pion dynamics in the vertex function is related to the pion rescattering diagram in the production operator. Therefore, at least close to threshold in 
Ref. [24], the most relevant mechanism that leads to inelasticities was missed.

As we saw, however, as we move away from the near-threshold region the contribution from the Delta isobar becomes more and more significant. Consequently, the results of Ref. [24] look significantly better at higher energies.

\section{Production of heavier mesons}

We start this section with some general remarks and then discuss some special examples. The phenomenology will be discussed only very briefly; for details on this we refer to the original references or the recent reviews $[1,2]$. The list of reactions discussed in detail is by no means complete. For example, we will not discuss here $\eta^{\prime}$ production, for this was already discussed in great detail in Ref. [2]. Neither will the production of vector mesons be discussed in detail. Here we refer to the recent contributions to the literature (Ref. [59] and references therein).

As can be seen from the headlines already, special emphasis will now be put on the physics that can be extracted from the particular reactions.

\subsection{Generalities}

As the mass of the produced meson increases, the initial energy needs to increase as well and consequently also the typical momentum transfer at threshold. This has various implications:

- the $N N$ interaction needed for a proper evaluation of the initial state interaction is less under control theoretically; for the $T=0$ channel there is not even a partial wave analysis available above the $\eta$-production threshold, due to a lack of data

- a larger momentum transfer makes it more difficult to construct the production operator; at least one should expect that the exchanges of heavier mesons become even more relevant compared to the pion case 
- it is not known, how to formulate a convergent effective field theory ${ }^{34}$

- less is known about the interactions of the subsystems

- the treatment of three-body singularities due to the exchange of light particles requires more care.

In the upcoming section we will not discuss in detail the role of the threebody singularities. Only recently was a method developed that in the future will allow inclusion of those singularities in calculations within the distorted wave born approximation [231]. This method was already applied in a toy model calculation [232], where its usefulness was demonstrated.

In calculations these singularities can occur only if the initial state interaction is included. For the production of mesons heavier than the $\eta$, however, no reliable model is available at present. Up to now no model for the production of heavy mesons takes three-body singularities into account, and thus their role is yet unclear.

\subsection{Remarks on the production operator for heavy meson production}

As in sec. 6 we will only look at analyses, that work within the distorted wave born approximation. For the two-baryon states nothing changes, other than that the nucleon-nucleon interaction needed for the initial state interaction gets less reliable from the phenomenological point of view as we go up in energy. The construction of the production operator, however, is now even more demanding, for with increasing momentum transfer heavier exchange mesons can play a significant role. Therefore, in order to get anything useful out of a model calculation, as many reaction channels as possible should be studied simultaneously. Only in this way can the phenomenological model parameters can be fixed and useful information be extracted. In this context also the simultaneous analysis of $p p$ and $p n$ induced reactions plays an important role,

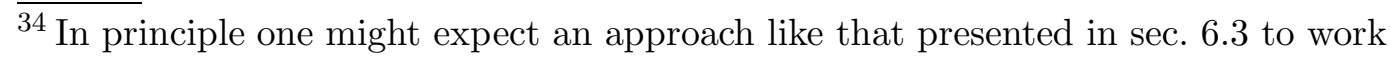
for the production of all Goldstone bosons. However, the next threshold after pion production is that for eta production and it already corresponds to an initial momentum of $p_{i}=770 \mathrm{MeV}$. Thus the expansion parameter would be $\sqrt{m_{\eta} / M_{N}}=0.8$.
} 
for different isospin structures in the production operator will lead to very different relative strengths of the two channels (c.f. discussion in sec. 1.5). Then, once a basic model is constructed that is consistent with most of the data, deviations in particular reaction channels can be studied.

In Ref. [34] the model described in sec. 6.2 was extended to $\eta$ production in nucleon-nucleon collisions, where the single channel $\pi N T$-matrix used in the pion production calculations was replaced by the multi channel mesonbaryon model of Ref. [233] to properly account for the exchange of heavy mesons. So far only $s$-waves were considered in the calculation. Results for the various total cross sections using two different models for the final state $N N$ interaction are shown in Fig. 32. The studies of Ref. [34] indicate, that a complete calculation for $N N \rightarrow N N \eta$ will put additional constraints on the relative phases of the various meson-baryon $\rightarrow \eta N$ transition amplitudes. Unfortunately, up to now the $\eta$ is the heaviest meson that can be investigated using this kind of microscopic approach, for there is no reliable model for the $N N$ interaction for energies significantly above the $\eta$ production threshold.

The strategy that therefore needs to be followed is to study many reaction channels consistently. This was done, for example, in a series of papers by Kaiser and others for $\pi, \eta, \eta^{\prime}$ [155], $\omega$ [234] as well as strangeness production [235] and by Nakayama and coworkers for $\omega[59,236,237], \phi[237,238]$, $\eta[239,240]$ and $\eta^{\prime}$ [241] production. Both groups construct the production operator as relativistic meson exchange currents, where the parameters are constrained by other data such as decay ratios. The striking difference between the two approaches is that the former studies $s$-waves only, does not consider effects of the initial state interaction and treats the final state interaction in an approximate fashion. The latter group includes the ISI through the procedure of Ref. [40] (c.f. sec. 3), treats the FSI microscopically and includes higher partial waves as well. It is important to stress that, where compatible, the two approaches give qualitatively similar results, as stressed in Ref. [234].

Recently it was observed that the onset of higher partial waves can strongly constrain the production operator [240]. In the same reference it was demonstrated that to unambiguously disentangle effects from FSI or higher partial 
waves, polarization observables are necessary. Thus one should expect that once a large amount of polarized data is available on the production of heavy mesons, the production operators and thus the relevant short-range physics for the production of a particular meson, can be largely fixed. As an example, this particular issue is discussed in detail in the next section.

\subsection{The reaction $N N \rightarrow N N \eta$ or properties of the $S_{11}(1535)$}

The $\eta$ meson is a close relative of the pion, since it is also a member of the nonet of the lightest pseudo scalar mesons. It is an isoscalar with a mass of 547.3 MeV - the difference in mass from the pion can be understood from its content of hidden strangeness through the Gell-Mann-Okubo mass formula $[242,243]$.

As for the pion, the $\eta$ couples strongly to a resonance, but a resonance with different quantum numbers: is it the positive parity $\Delta(1232)$ for the pion that in the $\Delta$ rest frame leads to $p$ wave pion production, the $\eta$ production is dominated by the negative parity $S_{11}(1535)^{35}$, which leads to $s$ wave $\eta$ production in its rest frame. As a consequence near-threshold $\eta$ production is completely dominated by the $S_{11}(1535)$ resonance. This statement can well be reversed: studying $\eta$ production in various reaction channels close to the production threshold allows selective study of the properties of the $S_{11}(1535)$ resonance in various environments. In this context it is interesting to note that the nature of this particular resonance is under discussion: within the so-called chiral unitary approach the resonance turns out to be dynamically generated [244,245], while on the other hand, detailed studies within the meson exchange approach [246], as described in sec. 6, as well as quark models (see Ref. [247] and references therein) call for a genuine quark resonance. One expects that a molecule behaves differently in the presence of other baryons than a threequark state due to the naturally enhanced affinity to meson baryon states of the former. In this sense data on $\eta$ production in few-baryon systems should

\footnotetext{
35 The quantum numbers are chosen in accordance with the partial wave in which the resonance would appear in $\pi N$ scattering. Therefore positive parity resonances appear in odd partial waves.
} 


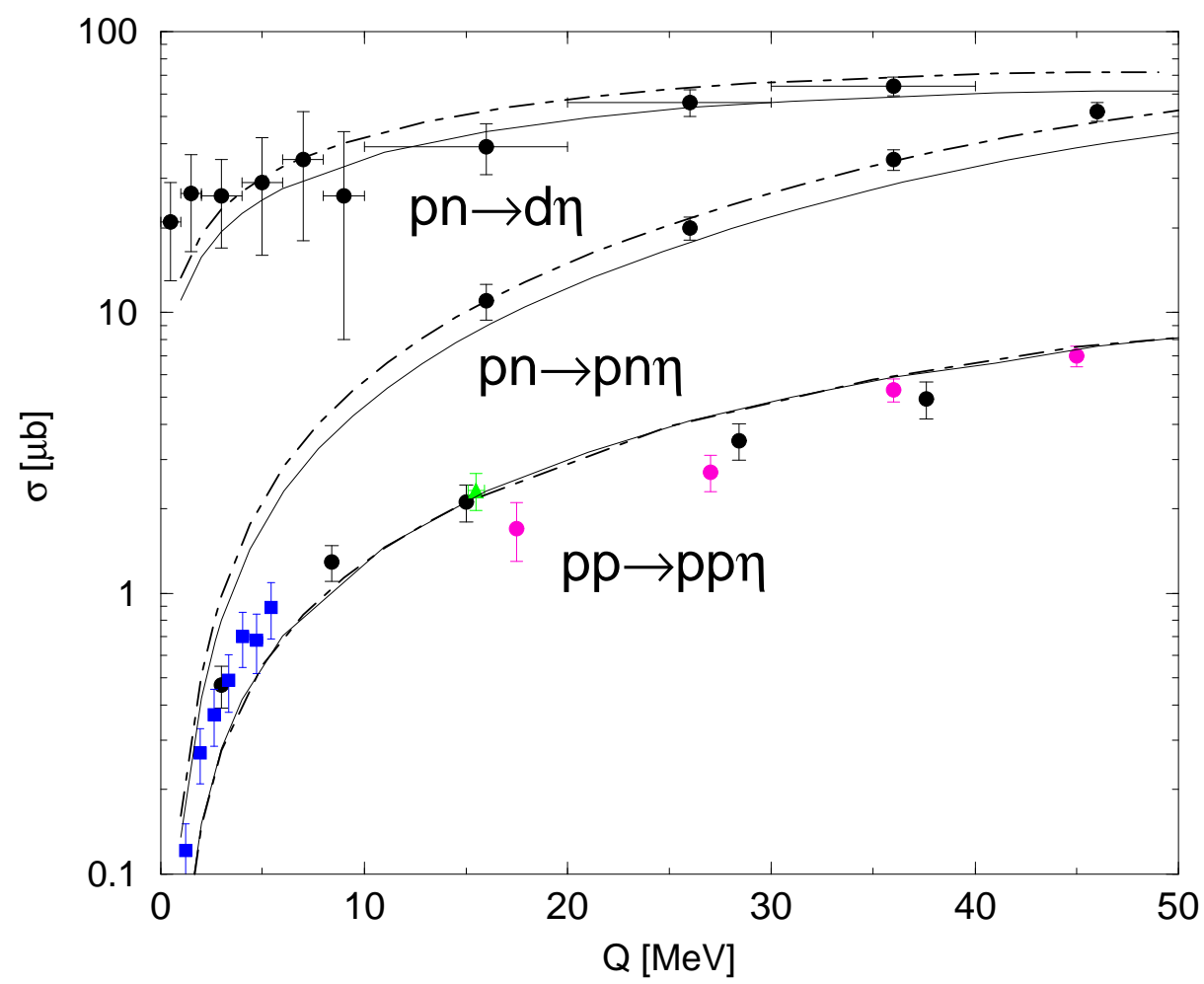

Fig. 32. Results for the total cross sections of the reactions $p p \rightarrow p p \eta, p n \rightarrow p n \eta$, and $p n \rightarrow d \eta$ from Ref. [34] employing different $N N$ models for the final state interaction. The solid lines represent the results with the CCF NN model [175] whereas the dashed-dotted lines were obtained for the Bonn B model [25]. Data are from Refs. [33,109-112,120].

provide valuable information about this lowest negative parity excitation of the nucleon.

The reaction $N N \rightarrow N N \eta$ was studied intensively both theoretically $[34,155,239,240,248-259]$ as well as experimentally (c.f. table 8) in recent years. The outstanding feature of $\eta$ production in $N N$ collisions is the strong effect of the $\eta N$ FSI, most visible in the reaction $p n \rightarrow d \eta$, where in a range of $10 \mathrm{MeV}$ the amplitude grows by about an order of magnitude [120]. This phenomenon was analyzed by means of Faddeev calculations, showing this enhancement to be consistent with a real part of the $\eta N$ scattering length of $0.4 \mathrm{fm}$ [248]. It is interesting that the same value of the $\eta N$ scattering length was recently extracted from an analyses of the reaction $\gamma d \rightarrow \eta p n[260]$ and that this value is consistent with that stemming from the microscopic model for meson-baryon scattering of Ref. [233]. First three body calculations for the reaction with 
a two-nucleon pair in the continuum show only a minor impact of the $\eta N$ interaction on the invariant mass spectra [261].

Most model calculations for the production operator share the property that the production operator was calculated within the meson exchange picture ${ }^{36}$. This was then convoluted with the $N N$ FSI, treated either microscopically or in some approximate fashion. In most analyses it was found that the dominant production mechanism is via the $S_{11}(1535)$, with the exception of Ref. [249], where the reaction $p p \rightarrow p p \eta$ turned out to be dominated by isoscalar meson exchanges, in analogy with the heavy meson exchanges in the reaction $p p \rightarrow p p \pi^{0}$. The largest qualitative differences between the various models is the relative importance of the exchanged mesons. In Refs. [155,252-255] the $\rho$-exchange turned out to be the dominant process, whereas it played a minor role in Refs. [34,256,249]. In Ref. [259] the $\rho$-exchange was not even considered, but $\eta$ exchange turned out to be significant there. It remains to be seen, however, if models that are dominated by isoscalar exchanges, Refs. $[259,249]$, are capable of accounting for the large ratio of $p n$ - to $p p$-induced eta production, as discussed in sec. 1.5.

As stressed above, in this section we do not want to focus on the details of the dynamics of the production operator, but instead on the physical aspect of what we can learn about the $S_{11}$ resonance from studying eta meson production in $N N$ collisions. Thus, in the remaining part of this section we will restrict ourselves to a model-independent analysis of a particular set of data based on the amplitude method introduced in sec. 4.3. Here we closely follow the reasoning of Ref. [240].

To be definite we will now concentrate on the measurement of angular distributions and invariant mass spectra for $p p \rightarrow p p \eta$ at $Q=15 \mathrm{MeV}$ [113]. The experiment shows that the angular distribution of both the two-proton pair and of the $\eta$ are flat, suggesting that only $s$-waves are present. On the other hand, the invariant mass distribution $d \sigma / d m_{p p}$ deviates significantly from what

\footnotetext{
$\overline{36}$ The only exception to the list given above is Ref. [257], where the ratio of $p p-$ to $p n$-induced $\eta$ production is explained by the instanton force. The relation between this result and the hadronic approach is unclear [257].
} 


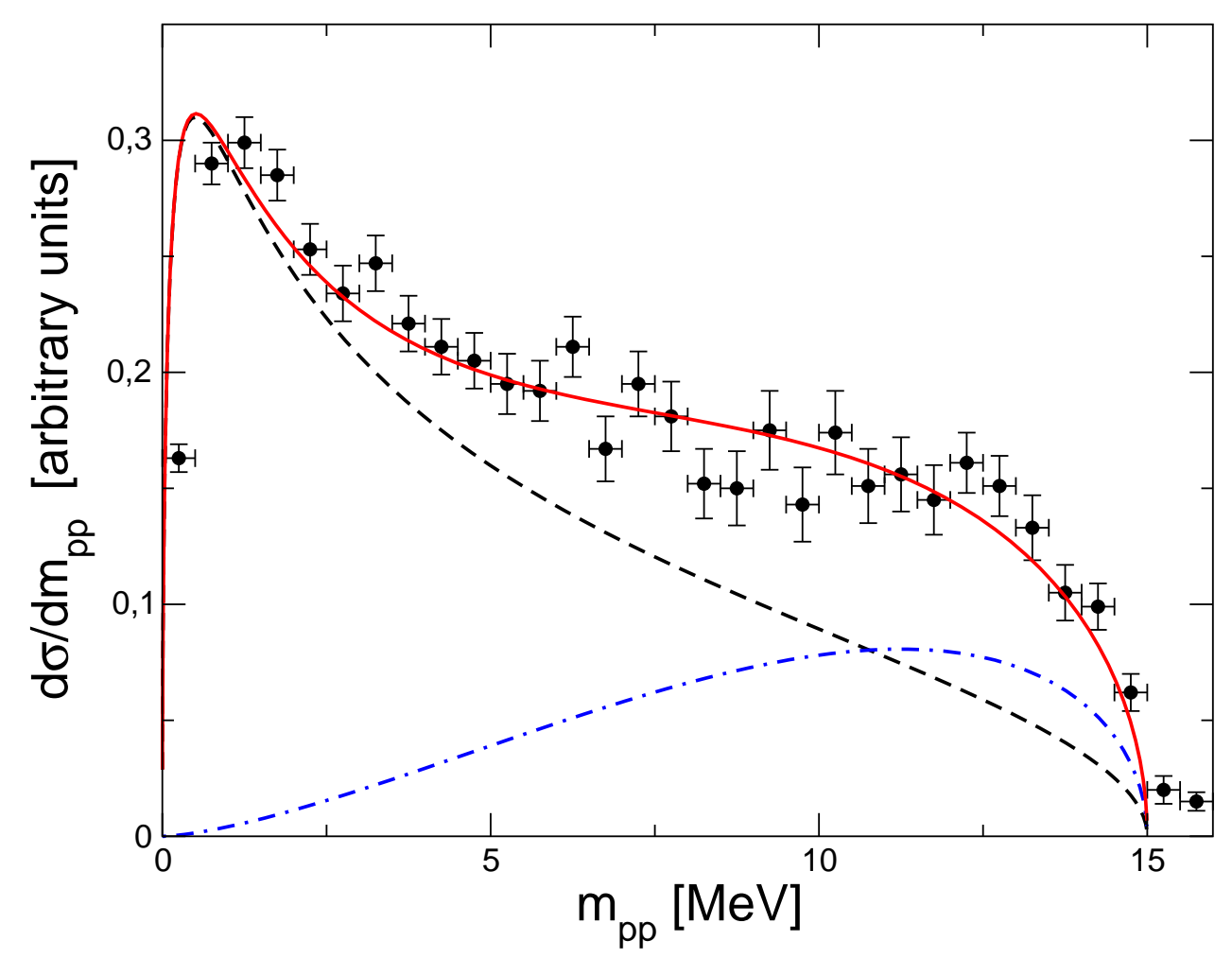

Fig. 33. Invariant mass spectrum for the two-proton system for the reaction $p p \rightarrow p p \eta$ at $Q=15 \mathrm{MeV}$. The dashed line shows the $S$ s contribution, the dot-dashed line the Ps distribution and the solid line the incoherent sum of both. The data is taken from Ref. [113].

is predicted based on the presence of the strong $p p$ FSI only (c.f. discussion of sec. 2). This is illustrated in Fig. 33, where the distribution for $p p S$-wave, $\eta s$-wave $(S s)$ is shown as the dashed line. Since the $\eta N$ interaction is known to be strong, its presence appears as the natural explanation for the deviation of the dashed line from the data. The structure, however, is even more pronounced at $Q=42 \mathrm{MeV}$ (c.f. Ref. [240]) in contrast to what should be expected for the $\eta N$ interaction.

On the other hand, the discrepancy between the data and the dashed curve can be well accounted for by a $P s$ configuration (given by phase-space times a factor of $p^{2}$, c.f. sec. 2.1). This is also illustrated in Fig. 33, where the pure $P s$ configuration is shown as the dashed-dotted line and the total result- - the incoherent sum of $P s$ and $S s$ cross section - as the solid line.

How is this compatible with the angular distribution of the two nucleon system 
being flat? This question can be very easily addressed in the amplitude method described in sec. 4.3. As long as we resrict ourselves to $\eta s$-waves and at most $P$ waves in the $N N$ system, the only non-vanishing terms in Eq. (48) are $\vec{Q}$, containing the amplitude for the $S s$ final state, and $\vec{A}$, containing the two possible amplitudes for the $P s$ final state. The explicit expressions for the amplitudes were given previously in Eqs. (55).

In this particular case we find for example (c.f. Eqs (49)-(54))

$$
\begin{aligned}
\sigma_{0} & =\frac{1}{4}\left(|\vec{Q}|^{2}+|\vec{A}|^{2}\right) \\
A_{0 i} \sigma_{0} & =0 \\
A_{x x} \sigma_{0} & =\frac{1}{4}\left(|\vec{Q}|^{2}-|\vec{A}|^{2}\right)
\end{aligned}
$$

where

$$
\begin{aligned}
& |\vec{Q}|^{2}=\left|a_{1}\right|^{2}, \\
& |\vec{A}|^{2}=p^{\prime 2}\left(\left|a_{2}-(1 / 3) a_{3}\right|^{2}+x^{2}\left\{\left|a_{3}\right|^{2}-(2 / 3) \operatorname{Re}\left(a_{3}^{*} a_{2}\right)\right\}\right),
\end{aligned}
$$

and $p^{\prime} x=\left(\vec{p}^{\prime} \cdot \hat{p}\right)$. Here the amplitudes $a_{1}, a_{2}$ and $a_{3}$ correspond to the transitions ${ }^{3} P_{0} \rightarrow{ }^{1} S_{0} s,{ }^{1} S_{0} \rightarrow{ }^{3} P_{0} s$, and ${ }^{1} D_{2} \rightarrow{ }^{3} P_{2} s$, respectively. From these equations we directly read that

- if $a_{3}$ is negligible, the $p p$ differential cross section $d \sigma / d x$ is flat

- the observable $\sigma_{0}\left(1-A_{x x}\right)$ directly measures the $N N P$-wave admixture

- any non-zero value for the analysing power is an indication for higher partial waves for the $\eta$.

It is easy to see that for $a_{3}=0$ the differential cross section has to be flat, since then the only partial wave that contributes to the $N N P$-waves is ${ }^{1} S_{0} \rightarrow{ }^{3} P_{0} s$ and a $J=0$ initial state does not contain any information about the beam direction.

Certainly, the $\eta N$ final state interaction has to be present in this reaction channel as well and it is important to understand its role in combination with the two-nucleon continuum state. It should be clear, however, that in order to understand quantitatively the role of the $\eta N$ interaction, it is important to 
pin down the $N N P$-wave contribution first. This is why a measurement of $A_{x x}$ for $p p \rightarrow p p \eta$ is so important.

What does it imply if the conjecture of a presence of $N N P$-waves at rather low excess energies were true? In Ref. [240] it is demonstrated that the need to populate predominantly the ${ }^{1} S_{0} \rightarrow{ }^{3} P_{0} s$ instead of the ${ }^{1} D_{2} \rightarrow{ }^{3} P_{2} s$ very strongly constrains the $N N \rightarrow S_{11} N$ transition potential. This indicates that a detailed study of $N N \rightarrow N N \eta$ should reveal information about the $S_{11}$ in a baryonic environment that might prove valuable in addressing the question of the nature of the lowest negative-parity nucleon resonance.

It should be mentioned that in Ref. [262] an alternative explanation for the shape of the invariant mass spectrum was given, namely an energy dependence was introduced to the production operator. Based on the arguments given in the previous chapters, we do not believe that this is a natural explanation. However, as we just outlined, with $A_{x x}$ an observable exists that allows to unambiguously distinguish between the two possible explanations. The experiment is possible at COSY [263].

\subsection{Associated strangeness production or the hyperon-nucleon interaction from production reactions}

In sec. 5 the small breaking of $S U(2)$ isospin symmetry present in the strong interaction was discussed. If we include strange particles in the analysis we can also study the breaking of flavor $S U(3)$. It is well-known that the light mesons and baryons can be arranged according to the irreducible representations of the group $S U(3)$. The mass splittings within the multiplet can be well accounted for by the number of strange quarks in some baryon or meson. However, not much is known about the dynamics of systems that contain strangeness. Many phenomenological models for, e.g., hyperon-nucleon scattering [268-271] use the flavor $S U(3)$ to fix the meson baryon-meson couplings. The remaining parameters, like the cut-off parameters, are then fit to the data. As we will discuss below, so far the existing data base for hyperon-nucleon scattering is insufficient to judge, if this procedure is appropriate. 

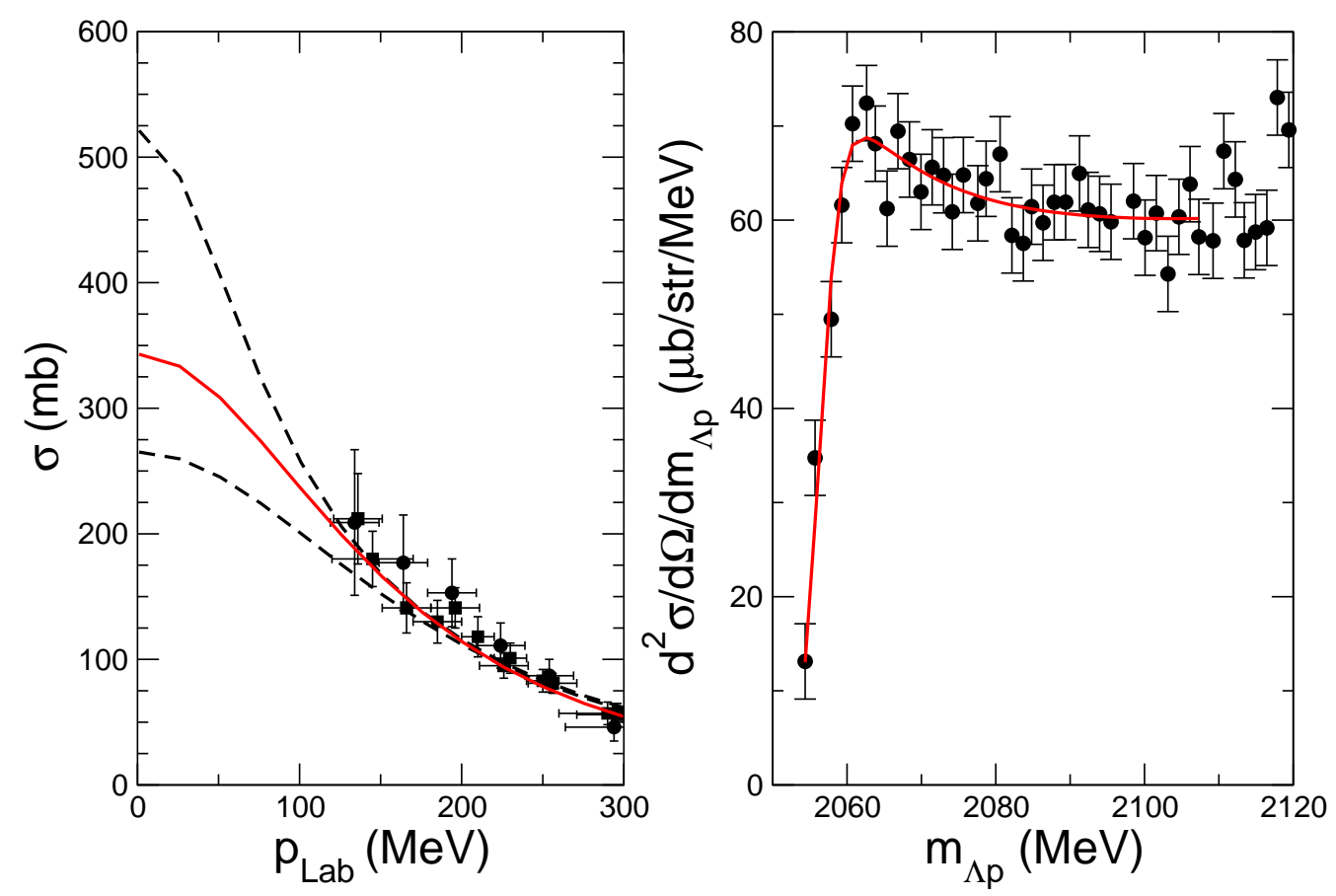

Fig. 34. Comparison of the quality of available data for the reactions $p \Lambda$ elastic scattering (data are from [264-266]) and $p p \rightarrow K^{+} \Lambda p$ at $T_{L a b}=2.3 \mathrm{GeV}$ [267] ${ }^{37}$. In both panels the red curve corresponds to a best fit to the data. In the left panel the dashed lines represent the spread in the energy behavior allowed by the data, according to the analysis of Ref. [266]; analogous curves in the right panel would lie almost on top of the solid line and are thus not shown explicitly.

As was stressed previously, effective field theories provide the bridge between the hadronic world and QCD. In connection with systems that contain strangeness there are still many open questions. Up to now it is not clear if the kaon is more appropriately treated as heavy or as light particle. In addition, in order to establish the counting rules it is important to know the value of the $S U(3)$ chiral condensate. For a review this very active field of research as well as the relevant references we refer to Ref. [272].

To further improve our understanding of the dynamics of systems that contain strangeness, better data is needed. The insights to be gained are relevant not only for few-body physics, but also for the formation of hypernuclei [273], and might even be relevant to the structure of neutron stars (for a recent discussion on the role of hyperons in the evolution of neutron stars see Ref. [274]). Naturally, the hyperon-nucleon scattering lengths are the quantities of interest in this context. 


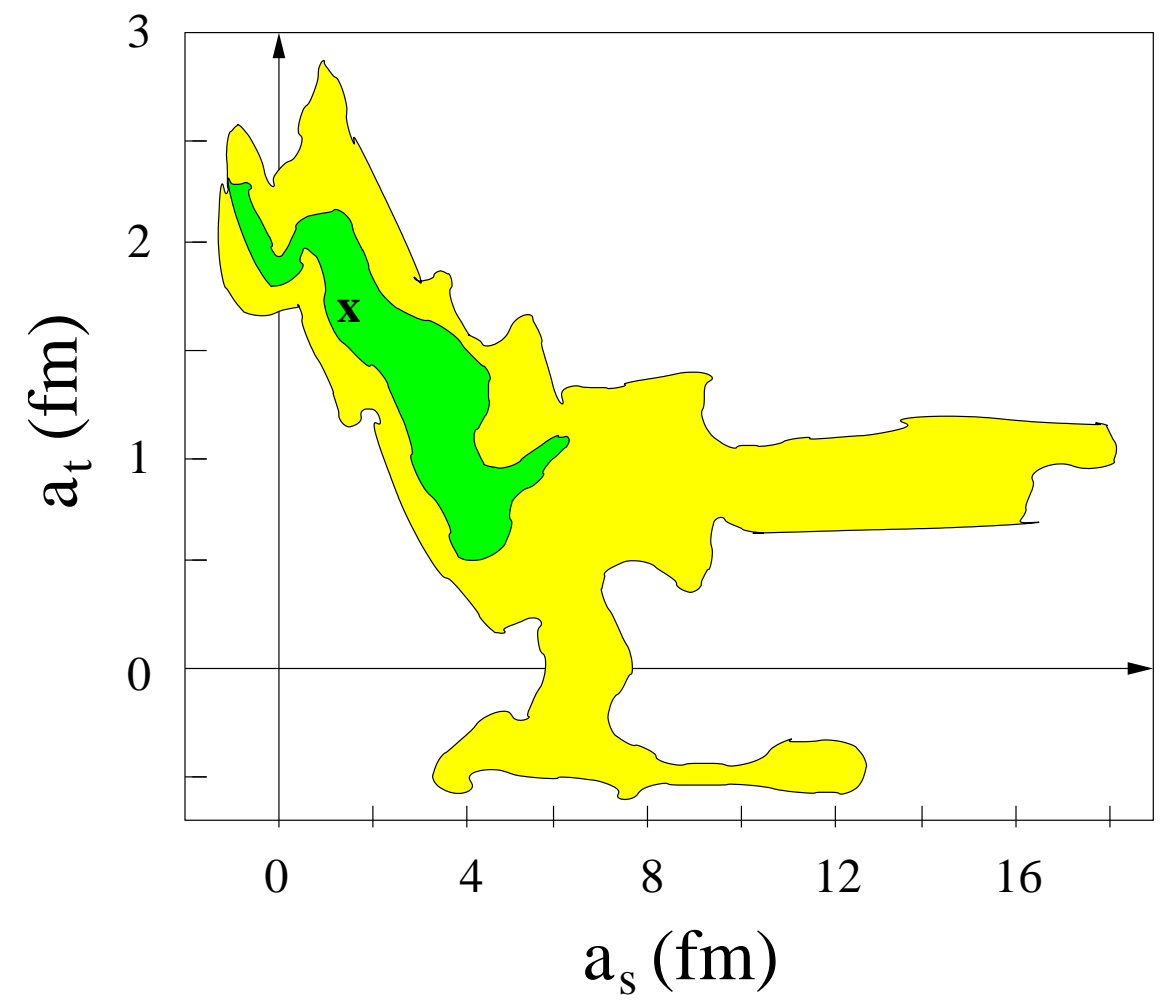

Fig. 35. Values allowed for the spin singlet and spin triplet scattering length by the $\Lambda N$ elastic scattering data according to Ref. [266]. The dark shaded area denotes the $1 \sigma$ range for the parameters and the light shaded area the $2 \sigma$ range. The cross shows the best fit value $\left(a_{s}=1.8 \mathrm{fm}\right.$ and $\left.a_{t}=1.6 \mathrm{fm}\right)$.

In the left panel of Fig. 34 we show the world data set for elastic $\Lambda N$ scattering. In Ref. [266] a Likelihood analysis based on the elastic scattering data was performed in order to extract the low energy $\Lambda N$ scattering parameters. The resulting contour levels are shown in Fig. 35, clearly demonstrating that the available elastic hyperon-nucleon scattering data do not significantly constrain the scattering lengths: the data allows for values of $(-1,2.3)$ as well as $(6,1)$ (all in fermi) for $\left(a_{s}, a_{t}\right)$ respectively. Later models were used to extrapolate the data. However, also in this way the scattering lengths could not be pinned down accurately. For example, in Ref. [269] one can find six different models that equally well describe the available data but whose ( $S$-wave) scattering lengths range from 0.7 to $2.6 \mathrm{fm}$ in the singlet channel and from 1.7 to $2.15 \mathrm{fm}$ in the triplet channel. Production reactions are therefore a promising alternative. In the literature the reactions $K^{-} d \rightarrow \gamma \Lambda n$ [9], $\gamma d \rightarrow K^{+} \Lambda n$ (Ref. [53] and references therein) and $p p \rightarrow p K^{+} \Lambda$ [122] were suggested. Therefore the method described in sec. 2.2, that applies to the latter two reactions, is an im- 
portant step towards a model-independent extraction of the hyperon-nucleon scattering lengths.

A natural question that arises is the quality of data needed e.g. for the reaction $p p \rightarrow p K^{+} \Lambda$ in order to significantly improve our knowledge about the hyperon-nucleon scattering lengths. In Ref. [32] it is demonstrated that data of the quality of the Saclay experiment for $p p \rightarrow K^{+} X$ [267], shown in right panel of Fig. $34{ }^{38}$ that had a mass resolution of $2 \mathrm{MeV}$, allows for an extraction of a scattering length with an experimental uncertainty of only $0.2 \mathrm{fm}$. Note, however, that the actual value of the scattering length extracted with Eq. (22) from those data is not meaningful, since the analysis presented can be applied only if just a single partial wave contributes to the invariant mass spectrum. The data set shown, however, represents the incoherent sum of the ${ }^{3} S_{1}$ and the ${ }^{1} S_{0}$ hyperon-nucleon final state.

The two spin states can be separated using polarization measurements. In sec. 4.3.1 as well as in Ref. [32] it was shown, that for the spin singlet final state the angular distributions of various polarization observables are largely constrained. This is sufficient to extract the spin dependence of the $\Lambda$-nucleon interaction from the reaction $p p \rightarrow p K^{+} \Lambda$. One more issue is important to stress: to make sure that the structure seen in the invariant mass spectrum truly stems from the final state interaction of interest, a Dalitz plot analysis is necessary, for resonances can well distort the spectra. This is discussed in detail in sec. 4.1 as well as at the end of sec. 2.2.

\subsection{Production of scalar mesons or properties of the lightest scalar}

The lightest scalar resonances $a_{0}(980)$ and $f_{0}(980)$ are two well-established states seen in various reactions [146], but their internal structure is still under discussion. Analyses can be found in the literature identifying these structures with conventional $q \bar{q}$ states (see Ref. [275] and references therein), compact $q q-\bar{q} \bar{q}$ states $[276,277]$ or loosely bound $K \bar{K}$ molecules [278,279]. In Ref. [280]

\footnotetext{
${ }^{38}$ Shown is only the low $m^{2}$ tail of the data taken at $T_{L a b}=2.3 \mathrm{GeV}$ and an angle of $10^{\circ}$
} 


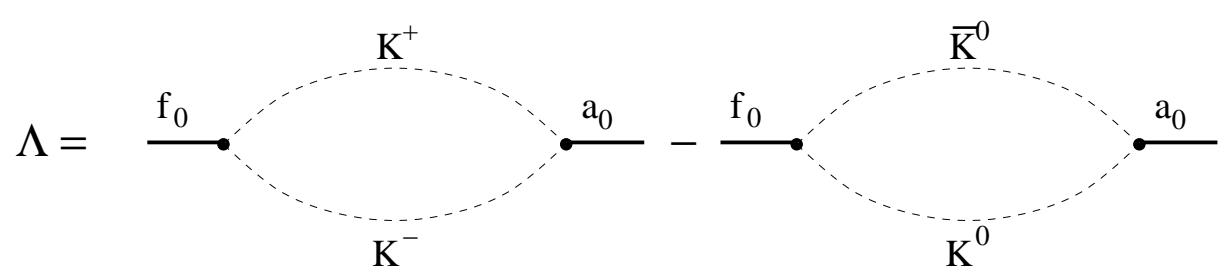

Fig. 36. Graphical illustration of the leading contribution to the $f_{0}-a_{0}$ mixing matrix element $\Lambda$ defined in Eq. (91).

a close connection between a possible molecule character of the light scalar mesons and chiral symmetry was stressed. It has even been suggested that at masses below 1.0 GeV a complete nonet of 4-quark states might exist [281].

Resolution of the nature of the light scalar resonances is one of the most pressing questions of current hadron physics. First of all we need to understand the multiplet structure of the light scalars in order to identify possible glueball candidates. In addition, it was pointed out recently that $a_{0}(980), f_{0}(980)$ as well as the newly discovered $D_{s}$ [282] might well be close relatives [283]. Thus, resolving the nature of the light scalar mesons allows one simultaneously to draw conclusions about the charmed sector and will also shed light on both the confining mechanism in light-light as well as light-heavy systems.

Although predicted long ago to be large [284], the phenomenon of $a_{0}-f_{0}$ mixing has not yet been established experimentally. In Ref. [284] it was demonstrated that the leading piece of the $f_{0}-a_{0}$ mixing amplitude can be written as $^{39}$

$$
\Lambda=\left\langle f_{0}|T| a_{0}\right\rangle=i g_{f_{0} K \bar{K}} g_{a_{0} K \bar{K}} \sqrt{s}\left(p_{K^{0}}-p_{K^{+}}\right)+\mathcal{O}\left(\frac{p_{K^{0}}^{2}-p_{K^{+}}^{2}}{s}\right)
$$

where $p_{K}$ denotes the modulus of the relative momentum of the kaon pair and the effective coupling constants are defined through $\Gamma_{x K \bar{K}}=g_{x K \bar{K}}^{2} p_{K}$. Obviously, this leading contribution is just that of the unitarity cut of the diagrams shown in Fig. 36 and is therefore model-independent. Note that in Eq. (91) electromagnetic effects were neglected, because they are expected to be small [284].

\footnotetext{
${ }^{39}$ Here we deviate from the original notation of Achasov et al. in order to introduce dimensionless coupling constants in line with the standard Flatté parameterization.
} 


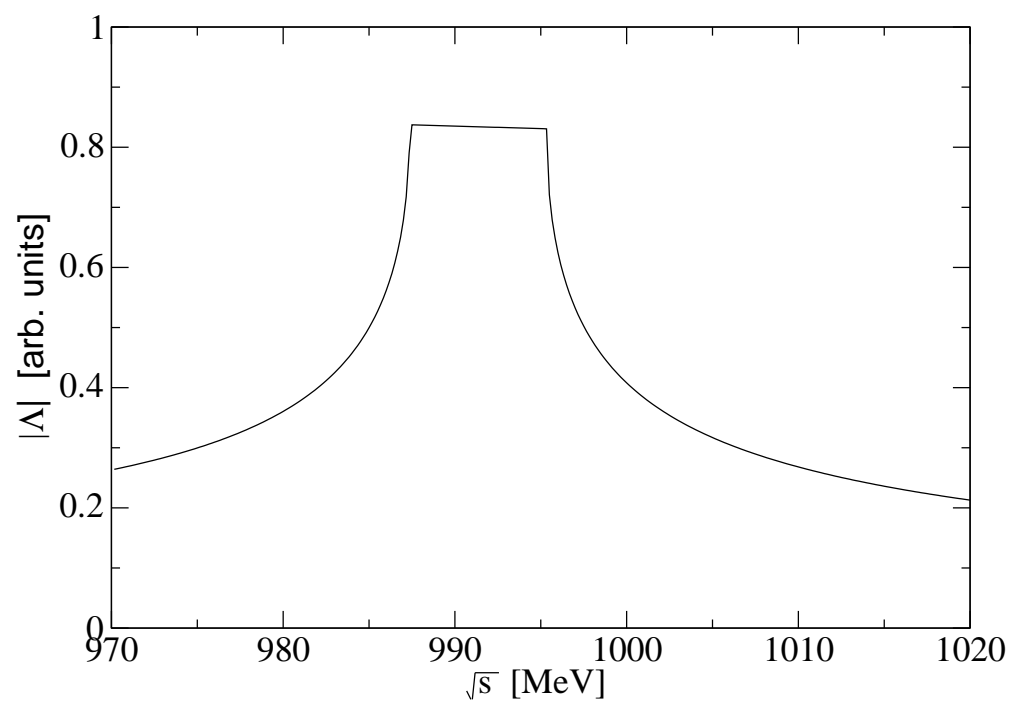

Fig. 37. Modulus of the leading piece of the mixing amplitude $\Lambda$ defined in Eq. (91). The two kinks occur at the $K^{+} K^{-}$(at 987.35 MeV) and the $\bar{K}^{0} K^{0}$ (995.34 MeV) threshold respectively.

The contribution shown in Eq. (91) is unusually enhanced between the $K^{+} K^{-}$ and the $\bar{K}^{0} K^{0}$ thresholds, a regime of only $8 \mathrm{MeV}$ width. Here it scales as (this formula is for illustration only - the Coulomb interaction contributes with similar strength to the kaon mass difference [285])

$$
\sqrt{\frac{m_{K^{0}}^{2}-m_{K^{+}}^{2}}{m_{K^{+}}^{2}+m_{K^{0}}^{2}}} \sim \sqrt{\frac{m_{u}-m_{d}}{\hat{m}+m_{s}}}
$$

where $m_{u}, m_{d}$ and $m_{s}$ denote the current quark mass of the up, down and strange quark, respectively, and $\hat{m}=\left(m_{u}+m_{d}\right) / 2$. This is in contrast to common CSB effects ${ }^{40}$ which scale as $\left(m_{u}-m_{d}\right) /\left(\hat{m}+m_{s}\right)$, since they have to be analytic in the quark masses. It is easy to see that away from the kaon thresholds $\Lambda$ returns to a value of natural size. This $\sqrt{s}$ dependence of $\Lambda$ is depicted in Fig. 37.

Within a microscopic model for $\pi \pi$ and $\bar{K} K$ scattering the mixing of $a_{0}$ and $f_{0}$ was studied in Ref. [286]. Within this model both resonances are of dynamical origin. The only mixing mechanism considered was the meson mass differences. Within this model the predictions of Ref. [284] were confirmed.

\footnotetext{
${ }^{40}$ Here we denote as common CSB effects those that occur at the Lagrangian level.
} 
As was demonstrated by Weinberg for the case of the deuteron [287], the effective couplings of resonances to the continuum states contain valuable information about the nature of the particles. In the case of the deuteron this analysis demonstrated that the effective coupling of the deuteron to the $p n$ continuum, as can be derived from the scattering length and the effective range, shows that the deuteron is a purely composite system. Recently it was demonstrated that, under certain conditions that apply in the case of $a_{0}$ and $f_{0}$, the analysis can be extended to unstable scalar states as well [288]. Accurate data on the effective couplings of the scalars to kaons should therefore provide valuable information about their nature. As was argued in sec. 5 , the occurrence of scalar mixing dominates the CSB effects in production reactions. Therefore quantifying the mixing matrix element might be one of the cleanest ways to measure the effective decay constants of $a_{0}$ and $f_{0}$. In sec. 5 it was demonstrated that from studies of $\pi \eta$ production in $N N$ and $d d$ collisions one should be able to extract the $a_{0}-f_{0}$ mixing amplitude. Those arguments were supported in sec. 4.3.2, where it was shown, that the reaction $p p \rightarrow d \bar{K}^{0} K^{+}$is indeed dominated by $\bar{K} K s$-waves. We should therefore expect a significant signal for the mixing as well. In the years to come we can thus expect the experimental information about the scalar mesons to be drastically improved.

\section{Meson production on light nuclei}

In this section we wish to make a few comments concerning meson production on light nuclei. Note, $d d$ induced meson production was discussed to some extent in section 5. A more extensive discussion can be found in Ref. [2].

\subsection{Generalities}

Almost all general statements made for meson production in $N N$ collisions apply equally well for meson production involving light nuclei. Naturally, now the selection rules are different (see discussion at the end of sec. 1.6) and the possible breakup of the nuclei introduces additional thresholds that must be 


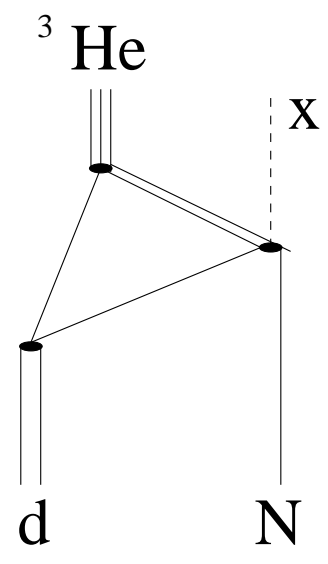

a)

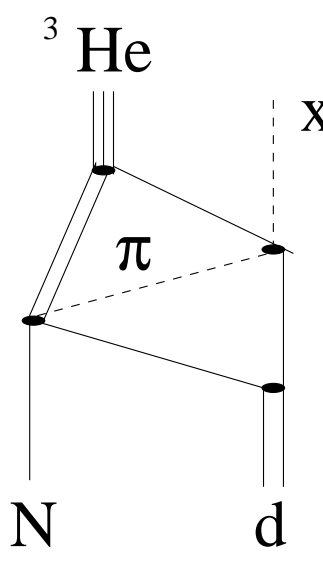

b)

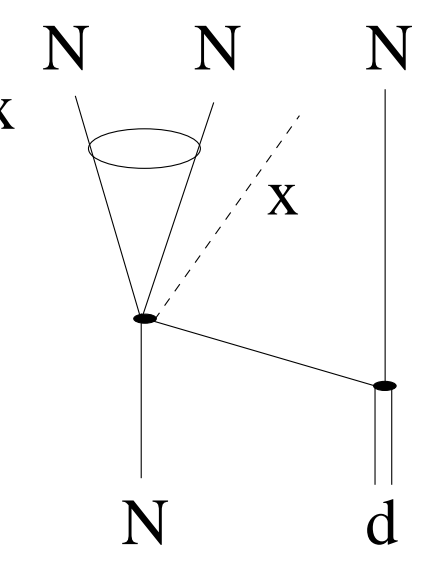

c)

Fig. 38. Possible diagrams to contribute to the reaction $N d \rightarrow 3 N x$. In diagram a) and b) the three nucleons in the final state from a bound state, whereas diagram c) shows the dominant diagram in quasi-free kinematics. Here it is assumed that only a particular nucleon pair (to be identified through proper choice of kinematics) interacts in the final state.

considered in theoretical analyses.

A comparison of meson production in two-nucleon collisions and in fewnucleon systems should improve our understanding of few-nucleon dynamics. As a result of a systematic study of, for example, both $N N$ and $p d$ induced reactions, a deeper insight into the importance of three-body forces should be gained. For example, a recent microscopic calculation using purely two-body input to calculate the reaction $\pi^{+3} \mathrm{He} \rightarrow p p p$, found that the data $[289,290]$ call for three-body correlations [291], confirming earlier studies [292]. It should be stressed, however, that this field is still in its infancy and a large amount theoretical effort is urgently called for.

\section{2 $2 \rightarrow 2$ reactions}

The reaction channel studied best up to date is $p d \rightarrow^{3}$ Hex. Data exist mainly from SATURNE for $x=\pi$ [293], $x=\omega$ [294], $x=\eta$ [295,296], as well as $x=\eta^{\prime}$ and $\phi[297]$. Most of the experiments were done using a polarized deuteron 
beam. In addition the reactions $d d \rightarrow \alpha \pi^{0}[140]^{41}$ and $d d \rightarrow \alpha \eta[298]$ were measured.

The reaction $p d \rightarrow^{3} \mathrm{He} \pi^{0}$ could be understood quantitatively from diagram a) of Fig. 38 [299]. However, it turned out that diagram a) alone largely under predicted the data $[300,301]$ for the reaction $p d \rightarrow^{3}$ He $\eta$, whereas diagram b) contributes sufficiently strongly to allow description of the data $[300,302,303]$. In all these approaches the individual amplitudes were taken from data directly; so far no microscopic calculation exists. The relevance of the pion exchange mechanism was explained in Ref. [304] as what the authors called a kinematic miracle: in the near-threshold regime the kinematics for eta production almost exactly matches that for $p p \rightarrow d \pi^{+}$followed by $\pi^{+} n \rightarrow \eta p$, with the intermediate pion on-shell.

The striking feature of reactions with an $\eta$-nucleus final state is the pronounced energy dependence that was already described in sec. 7.3 for the reaction $p n \rightarrow d \eta$ - often interpreted as a signal of an existing (quasi)bound state in the $\eta$-nucleus system. Indeed, since the quark structure of the $\eta \sim \bar{u} u+\bar{d} d$ is proportional to the number operator, one should expect the $\eta$-nucleus interaction to get stronger with increasing number of light quark flavors present in the interaction region. For a more detailed discussion of this issue we refer to Ref. [2].

\subsection{Quasi-free Production}

A deuteron is a loosely bound state of a proton and a neutron. For properly chosen kinematics, the deuteron can therefore be viewed as an effective neutron beam/target as alternative to neutron beams (see Ref. [2] and references therein). The corresponding diagram is shown in Fig. 38c).

Obviously, the existence of a three-nucleon bound states already shows that there must be a kinematic regime, where the interaction of all three nucleons in the final state is significant, namely, when all three have small relative

\footnotetext{
${ }^{41}$ Note that this reaction is charge symmetry breaking and was discussed in sec. 5 .
} 


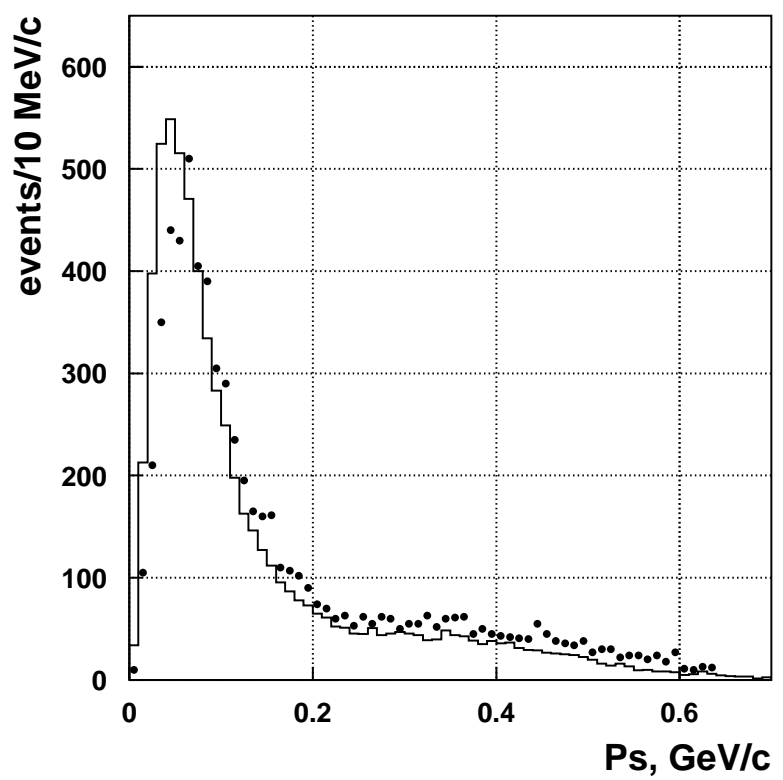

Fig. 39. Momentum distribution of proton-spectators in the reaction $\bar{p} d \rightarrow 3 \pi^{+} 2 \pi^{-} p$. The data are taken from Ref. [305] while the solid line is is based on a calculation that includes the quasi-free production as well as meson rescattering. The figure is taken from [306], where also details about the corresponding calculation can be found.

momenta. On the other hand, if the spectator nucleon escapes completely unaffected, its momentum distribution should be given by just half the deuteron momentum, convoluted with the deuteron wavefunction times the phase-space factors. Thus, we should expect a momentum distribution for the spectator that shows in addition to a pronounced peak from the quasi-free production a long tail stemming from rescattering in the final state.

Experimental data exist for the proton-spectator momentum distribution for the reaction $\bar{p} d \rightarrow 3 \pi^{+} 2 \pi^{-} p$ [305]. In Fig. 39 this data is shown together with the results of a calculation [306] that considers both the quasi-free piece as well as a rescattering piece. The data clearly shows the quasi-elastic peak. Thus, when considering spectator momenta that are of about $100 \mathrm{MeV}$ or less, to a good approximation, the reaction should be quasi-free.

Experimentally this conjecture can be checked by comparing data for $p p$ induced reactions with those stemming from a $p d$ initial state with a neutron 
spectator. Those comparisons were carried out at TRIUMF for pion production $[74,75]$ as well as at CELSIUS for $\eta$ production $[33,120]$, showing that the quasi-elastic assumption is a valid approximation.

In sec. 5 it was argued that a forward-backward asymmetry in the reaction $p n \rightarrow d\left(\pi^{0} \eta\right)_{s-w a v e}$ is a good system from which to extract the $f_{0}-a_{0}$ mixing matrix element. If investigated at COSY, this reaction can only be studied with a deuteron as effective neutron target. Since we are after a CSB effect, the expected asymmetry is of the order of a few percent. Thus we need to ensure a priori that the spectator does not introduce an asymmetry of this order through its strong interaction with, say, the deuteron. Theoretically it would be very demanding to control such a small effect in a four-particle system. Fortunately, we can test experimentally to what extent the spectator introduces a forward-backward asymmetry by studying, for example, the reaction $p d \rightarrow \pi^{+} d n$. If the reaction were purely quasi-elastic, the angular distribution of the $\pi^{+} d$ system in its rest frame would be forward-backward symmetric, for it would be stemming from a $p p$ initial state. However, any interaction of the spectator with either the pion or the deuteron, should immediately introduce some forward-backward asymmetry. Experimental investigations of this very sensitive test of the spectator approach are currently under way [307].

\section{Summary}

The physics of meson production in nucleon-nucleon collisions is very rich. The various observables that are nowadays accessible experimentally are, for example, influenced by baryon resonances and final state interactions as well as their interference. It is therefore inevitable to use polarized observables to disentangle the many different physics aspects.

From the authors personal point of view, the most important issues for the field are:

- that $N N$ and $d d$ induced reactions are very well suited for studies of charge-symmetry breaking [5]. Especially, investigation of the reactions 
$p n \rightarrow d\left(\pi^{0} \eta\right)_{s-\text { wave }}$ and $d d \rightarrow \alpha\left(\pi^{0} \eta\right)_{s-\text { wave }}$ should shed light on the nature of the scalar mesons. For the experiments planned in this context we refer to Ref. [308];

- that sufficiently strong final state interactions can be extracted from production reactions with large momentum transfer, as, for example, $p p \rightarrow p K^{+} \Lambda$. The condition for an accurate extraction is a measurement with high resolution, as should be possible with the HIRES experiment at COSY [309];

- that an effective field theory was developed for large momentum transfer reactions such as $N N \rightarrow N N \pi$. Once pushed to sufficiently high orders, those studies will not only provide us with a better understanding of the phenomenology of meson production in $N N$ collisions but also allow us to identify the charge symmetry breaking operators that lead to the cross sections reported in Refs. [6,140];

- that resonances can be studied in $N N$ induced reactions. Those studies are complementary to photon induced reactions, since the resonances can be excited by additional mechanisms, which can be selected using, for example, spin 0 particles as spin filter [4]. In addition, also the properties of resonances in the presence of another baryon can be systematically investigated.

There are very exciting times to come in the near future, for not only does the improvement of experimental apparatus in recent years permit the acquisition of a great deal of high-precision data but also because or theoretical understanding has now also improved to a point that important physics questions can be addressed systematically.

\section{Acknowledgments}

This article would not have been possible without the strong support, the lively discussions as well as the fruitful collaborations of the author with V. Baru, M. Büscher, J. Durso, Ch. Elster, A. Gasparyan, J. Haidenbauer, B. Holstein, V. Kleber, O. Krehl, N. Kaiser, S. Krewald, B. Kubis, A.E. Kudryavtsev, U.G. Meißner, K. Nakayama, J. Niskanen, A. Sibirtsev, and J. Speth. Thanks to all of you! I am especially grateful for the numerous editorial remarks by J. Durso. 


\section{Appendices}

\section{A Kinematical variables}

In this report we only deal with reactions, in which the energies of the final states are so low that a non-relativistic treatment of the baryons is justified. This greatly simplifies the kinematics. Thus, in the center of mass system we may write for the reaction $N N \rightarrow B_{1} B_{2} x$,

$$
\begin{aligned}
& E_{t o t}=2 \sqrt{\vec{p}^{2}+M_{N}^{2}} \\
& =\sqrt{M_{1}^{2}+{p_{1}^{\prime}}^{2}}+\sqrt{M_{2}^{2}+{p_{2}^{\prime}}^{2}}+\sqrt{m_{x}^{2}+{q^{\prime}}^{2}} \\
& \approx M_{1}+M_{2}+\frac{p^{\prime 2}}{2 \mu_{12}}+\frac{q^{\prime 2}}{2\left(M_{1}+M_{2}\right)}+\sqrt{m_{x}^{2}+q^{\prime 2}} \text {, }
\end{aligned}
$$

where $p$ denotes the momentum of the initial nucleons, $p^{\prime}$ the relative momentum of the final nucleons and $q^{\prime}$ the center of mass momentum of the meson of mass $m_{x}$. The reduced mass of the outgoing two baryon system is denoted by $\mu_{12}=M_{1} M_{2} /\left(M_{1}+M_{2}\right)$.

The kinematical variable traditionally used for the total energy of a meson

production reaction is $\eta$, the maximum meson momentum in units of the meson mass. Then one gets from Eq. (A.1):

$$
E_{t o t}(\eta) \approx M_{1}+M_{2}+\eta^{2} \frac{m_{x}^{2}}{2\left(M_{1}+M_{2}\right)}+m_{x} \sqrt{1+\eta^{2}}
$$

Another often used variable for the energy of a meson production reaction is

$$
Q=\sqrt{s}-\left(M_{1}+M_{2}+m_{x}\right) .
$$

It is straightforward to express $\eta$ in terms of $Q$ :

$$
\begin{aligned}
& \eta=\frac{1}{2 m_{x}} \sqrt{\frac{\left(s-M_{f}^{2}-m_{x}^{2}\right)^{2}-4\left(m_{x} M_{f}\right)^{2}}{s}} \\
& \simeq \frac{1}{m_{x}} \sqrt{2 \mu Q}\left(1+\frac{Q}{4 \mu}\left(1-3 \frac{\mu}{M_{f}+m_{x}}\right)\right) .
\end{aligned}
$$




\begin{tabular}{|c|c|c|c|c|c|c|}
\hline \multirow[b]{2}{*}{$\eta$} & \multicolumn{2}{|c|}{$p p \rightarrow d \pi^{+}$} & \multicolumn{2}{|c|}{$p p \rightarrow p p \pi^{0}$} & \multicolumn{2}{|c|}{$p p \rightarrow p n \pi^{+}$} \\
\hline & $T_{L a b}$ & $Q$ & $T_{L a b}$ & $Q$ & $T_{L a b}$ & $Q$ \\
\hline 0.00 & 287.6 & 0.00 & 279.6 & 0.00 & 292.3 & 0.00 \\
\hline 0.05 & 288.0 & 0.19 & 280.0 & 0.18 & 292.7 & 0.19 \\
\hline 0.10 & 289.2 & 0.75 & 281.2 & 0.72 & 293.9 & 0.75 \\
\hline 0.15 & 291.2 & 1.68 & 283.1 & 1.62 & 295.9 & 1.68 \\
\hline 0.20 & 293.9 & 2.97 & 285.8 & 2.87 & 298.7 & 2.97 \\
\hline 0.25 & 297.5 & 4.62 & 289.2 & 4.46 & 302.3 & 4.62 \\
\hline 0.30 & 301.8 & 6.61 & 293.3 & 6.38 & 306.6 & 6.61 \\
\hline 0.35 & 306.8 & 8.94 & 298.1 & 8.62 & 311.6 & 8.94 \\
\hline 0.40 & 312.5 & 11.58 & 303.6 & 11.17 & 317.3 & 11.58 \\
\hline 0.45 & 318.9 & 14.53 & 309.8 & 14.02 & 323.7 & 14.53 \\
\hline 0.50 & 325.9 & 17.77 & 316.5 & 17.14 & 330.7 & 17.77 \\
\hline 0.55 & 333.5 & 21.29 & 323.9 & 20.53 & 338.3 & 21.29 \\
\hline 0.60 & 341.7 & 25.06 & 331.8 & 24.18 & 346.6 & 25.06 \\
\hline 0.65 & 350.5 & 29.09 & 340.2 & 28.05 & 355.3 & 29.08 \\
\hline 0.70 & 359.8 & 33.34 & 349.1 & 32.16 & 364.6 & 33.34 \\
\hline 0.75 & 369.5 & 37.81 & 358.5 & 36.47 & 374.4 & 37.81 \\
\hline 0.80 & 379.8 & 42.49 & 368.4 & 40.98 & 384.6 & 42.48 \\
\hline 0.85 & 390.5 & 47.36 & 378.6 & 45.67 & 395.3 & 47.35 \\
\hline 0.90 & 401.6 & 52.40 & 389.3 & 50.54 & 406.5 & 52.40 \\
\hline 0.95 & 413.1 & 57.62 & 400.4 & 55.57 & 418.0 & 57.62 \\
\hline 1.00 & 425.0 & 63.00 & 411.8 & 60.75 & 429.9 & 62.99 \\
\hline 1.10 & 449.8 & 74.19 & 435.7 & 71.54 & 454.8 & 74.18 \\
\hline 1.20 & 476.0 & 85.91 & 460.9 & 82.83 & 481.0 & 85.90 \\
\hline 1.30 & 503.4 & 98.10 & 487.1 & 94.57 & 508.4 & 98.09 \\
\hline 1.40 & 531.9 & 110.71 & 514.5 & 106.72 & 536.9 & 110.69 \\
\hline 1.50 & 561.4 & 123.69 & 542.8 & 119.23 & 566.4 & 123.68 \\
\hline
\end{tabular}

Table A.1

The values for $T_{L a b}, Q$ and $\eta$ for the different channels for pion production.

where $M_{f}=M_{1}+M_{2}$ and the reduced mass of the full system is given by $\mu=M_{f} m_{x} /\left(M_{f}+m_{x}\right)$. For the latter approximation we used that in the close to threshold regime $Q \ll\left(M_{f}+m_{x}\right)$.

Traditionally $\eta$ is used for the pion production only, whereas $Q$ is used for the production of all heavier mesons. To simplify the comparison of results for 
those different reaction channels, we present in table A.1 the various values for $\eta, Q$ as well as $T_{L a b}$ for the different pion production channels.

For completeness we also give the relation to the Laboratory variables

$$
\begin{aligned}
& T_{L a b}=\frac{s-4 M^{2}}{2 M}, \\
& p_{L a b}^{2}=T_{L a b}^{2}+2 M T_{L a b} .
\end{aligned}
$$

\section{B Collection of useful formulas}

\section{B.1 Definition of Coordinate system}

In this appendix we give the explicit expressions for the vectors relevant for the description of a general $2 \rightarrow 3$ reaction. We work in the coordinate system, where the $z$-axis is given by the beam momentum $\vec{p}$. These formulas are particularly useful for sec. 4.3. Thus we have

$\vec{p}=p\left(\begin{array}{l}0 \\ 0 \\ 1\end{array}\right), \quad \vec{p}^{\prime}=p^{\prime}\left(\begin{array}{c}\sin \left(\theta_{p^{\prime}}\right) \cos \left(\phi_{p^{\prime}}\right) \\ \sin \left(\theta_{p^{\prime}}\right) \sin \left(\phi_{p^{\prime}}\right) \\ \cos \left(\theta_{p^{\prime}}\right)\end{array}\right), \quad \vec{q}^{\prime}=q^{\prime}\left(\begin{array}{c}\sin \left(\theta_{q^{\prime}}\right) \cos \left(\phi_{q^{\prime}}\right) \\ \sin \left(\theta_{q^{\prime}}\right) \sin \left(\phi_{q^{\prime}}\right) \\ \cos \left(\theta_{q^{\prime}}\right)\end{array}\right)$

From these one easily derives

$$
\begin{aligned}
& i\left(\vec{p} \times \vec{p}^{\prime}\right)=i p p^{\prime}\left(\begin{array}{c}
-\sin \left(\theta_{p^{\prime}}\right) \sin \left(\phi_{p^{\prime}}\right) \\
\sin \left(\theta_{p^{\prime}}\right) \cos \left(\phi_{p^{\prime}}\right) \\
0
\end{array}\right), \\
& i\left(\vec{p}^{\prime} \times \vec{q}^{\prime}\right)=i p^{\prime} q^{\prime}\left(\begin{array}{c}
\cos \left(\theta_{q^{\prime}}\right) \sin \left(\theta_{p^{\prime}}\right) \sin \left(\phi_{p^{\prime}}\right)-\cos \left(\theta_{p^{\prime}}\right) \sin \left(\theta_{q^{\prime}}\right) \sin \left(\phi_{q^{\prime}}\right) \\
-\cos \left(\theta_{q^{\prime}}\right) \sin \left(\theta_{p^{\prime}}\right) \cos \left(\phi_{p^{\prime}}\right)+\cos \left(\theta_{p^{\prime}}\right) \sin \left(\theta_{q^{\prime}}\right) \cos \left(\phi_{q^{\prime}}\right) \\
-\sin \left(\theta_{p^{\prime}}\right) \sin \left(\theta_{q^{\prime}}\right) \sin \left(\phi_{q^{\prime}}-\phi_{p^{\prime}}\right)
\end{array}\right),
\end{aligned}
$$

as well as the analogous expression for $i\left(\vec{p} \times \vec{q}^{\prime}\right)$. 


\section{B.2 Spin traces}

In this appendix some relations are given, that are useful to evaluate expression that arise from in the amplitude method appearing described in sec. 4.3.

$$
\sigma_{y} \chi_{i}^{*} \chi_{i}^{T} \sigma_{y}=\frac{1}{2} \sigma_{y}\left(1+\vec{P}_{i} \cdot \vec{\sigma}^{T}\right) \sigma_{y}=\frac{1}{2}\left(1-\vec{P}_{i} \cdot \vec{\sigma}\right)
$$

The sum over the spins of the external particles leads to traces in spin space, such as

$$
\begin{aligned}
\operatorname{tr}\left(\sigma_{i}\right) & =0 \\
\operatorname{tr}\left(\sigma_{i} \sigma_{j}\right) & =2 \delta_{i j} \\
\operatorname{tr}\left(\sigma_{i} \sigma_{\beta} \sigma_{j}\right) & =2 i \epsilon_{i \beta j} \\
\operatorname{tr}\left(\sigma_{\alpha} \sigma_{i} \sigma_{\beta} \sigma_{j}\right) & =2\left(\delta_{i \alpha} \delta_{j \beta}+\delta_{j \alpha} \delta_{i \beta}-\delta_{i j} \delta_{\alpha \beta}\right)
\end{aligned}
$$

To identify dependent structures the following reduction formula is useful

$$
\begin{aligned}
\vec{a}^{2} \vec{b} \cdot(\vec{c} \times \vec{d}) & = \\
(\vec{a} \cdot \vec{b}) & \vec{a} \cdot(\vec{c} \times \vec{d})+(\vec{a} \cdot \vec{d}) \vec{a} \cdot(\vec{b} \times \vec{c})+(\vec{a} \cdot \vec{c}) \vec{a} \cdot(\vec{d} \times \vec{b}) .
\end{aligned}
$$

To recall the sign or, better, the order of the vectors appearing, observe that on the right hand side the vectors other than $\vec{a}$ are rotated in cyclic order. To prove Eq. (B.7) we use

$$
\begin{aligned}
\delta_{i j} \epsilon_{k l m}-\delta_{i m} \epsilon_{k l j} & =\epsilon_{k l \gamma}\left(\delta_{i j} \delta_{m \gamma}-\delta_{i m} \delta_{j \gamma}\right) \\
& =\epsilon_{k l \gamma} \epsilon_{i \gamma \alpha} \epsilon_{j m \alpha} \\
& =\epsilon_{j m \alpha}\left(\delta_{k \alpha} \delta_{l i}-\delta_{k i} \delta_{l \alpha}\right)=\delta_{i l} \epsilon_{j m k}-\delta_{k i} \epsilon_{j m l}
\end{aligned}
$$

\section{Partial wave expansion}

In this appendix we give the explicit relations between the partial wave amplitudes for reactions of the type $N N \rightarrow N N x$ and the spherical tensors defined in Eq. (34), where $x$ is a scalar particle. The relations between the spherical tensors and the various observables is given in tables 4 and 5 . 
In terms of the partial wave amplitudes, we can write for two spin- $\frac{1}{2}$ particles in the initial state

$$
\begin{aligned}
T_{k_{1} q_{1}, k_{2} q_{2}}^{k_{3} q_{3}, k_{4} q_{4}}=\frac{1}{16 \pi} \sum & \left\langle S^{\prime} M_{S}^{\prime}, \vec{p}^{\prime}, \vec{q}^{\prime}|M| S M_{S}, \vec{p}\right\rangle\left\langle\bar{S} \bar{M}_{S}, \vec{p}\left|M^{\dagger}\right| \bar{S}^{\prime} \bar{M}_{S}^{\prime}, \vec{p}^{\prime}, \vec{q}^{\prime}\right\rangle \\
& \times\left\langle S M_{S}\left|\tau_{k_{1} q_{1}}^{(b)} \tau_{k_{2} q_{2}}^{(t)}\right| \bar{S} \bar{M}_{S}\right\rangle\left\langle\bar{S}^{\prime} \bar{M}_{S}^{\prime}\left|\tau_{k_{3} q_{3}}^{\left(f_{1}\right) \dagger} \tau_{k_{4} q_{4}}^{\left(f_{2}\right) \dagger}\right| S^{\prime} M_{S}^{\prime}\right\rangle \\
=\frac{1}{4} \sum \sqrt{\frac{(2 \bar{L}+1)(2 L+1)}{(2 \bar{J}+1)(2 J+1)}} & \times\left\langle S^{\prime} M_{S}^{\prime}, L^{\prime} M_{L}^{\prime} \mid j^{\prime} M_{j}^{\prime}\right\rangle\left\langle j^{\prime} M_{j}^{\prime}, l^{\prime} m_{l}^{\prime} \mid J M_{J}\right\rangle\left\langle S M_{S}, L 0 \mid J M_{J}\right\rangle \\
& \times\left\langle S^{\prime} M_{S}^{\prime}, \bar{L}^{\prime} \bar{M}_{L}^{\prime} \mid \bar{j}^{\prime} \bar{M}_{j}^{\prime}\right\rangle\left\langle\bar{j}^{\prime} \bar{M}_{j}^{\prime}, \bar{l}^{\prime} \bar{m}_{l}^{\prime} \mid \bar{J} \bar{M}_{J}\right\rangle\left\langle\bar{S} \bar{M}_{S}, \bar{L} 0 \mid \bar{J} \bar{M}_{J}\right\rangle \\
& \times\left\langle S M_{S}\left|\tau_{k_{1} q_{1}}^{(b)} \tau_{k_{2} q_{2}}^{(t)}\right| \bar{S} \bar{M}_{S}\right\rangle\left\langle\bar{S}^{\prime} \bar{M}_{S}^{\prime}\left|\tau_{k_{3} q_{3}}^{\left(f_{1}\right) \dagger} \tau_{k_{4} q_{4}}^{\left(f_{2}\right) \dagger}\right| S^{\prime} M_{S}^{\prime}\right\rangle \\
& \times Y_{l^{\prime} m_{l}^{\prime}}\left(\hat{q}^{\prime}\right) Y_{L^{\prime} M_{L}^{\prime}}\left(\hat{p}^{\prime}\right) Y_{\bar{l}^{\prime} \bar{m}_{l}^{\prime}}\left(\hat{q}^{\prime}\right)^{*} Y_{\bar{L}^{\prime} \bar{M}_{L}^{\prime}}\left(\hat{p}^{\prime}\right)^{*} \\
& \times M^{\alpha}(s, \epsilon) M^{\bar{\alpha}}(s, \epsilon)^{\dagger} .
\end{aligned}
$$

In order to proceed the following identities are useful [310]:

$$
\begin{aligned}
Y_{l_{1} m_{1}}(\hat{p}) Y_{l_{2} m_{2}}(\hat{p})=\sum_{l m} \sqrt{\frac{\left(2 l_{1}+1\right)\left(2 l_{2}+1\right)}{(2 l+1) 4 \pi}} \\
\\
\quad \times\left\langle l_{1} m_{1}, l_{2} m_{2} \mid l m\right\rangle\left\langle l_{1} 0, l_{2} 0 \mid l 0\right\rangle Y_{l m}(\hat{p}) \\
\left\langle\sigma\left|\tau_{k q}\right| \sigma^{\prime}\right\rangle=(-)^{q} \sqrt{2 k+1}\left\langle\frac{1}{2} \sigma, k(-q) \mid \frac{1}{2} \sigma^{\prime}\right\rangle .
\end{aligned}
$$

The latter, for instance, allows evaluation of the matrix element of the spin operators: 


$$
\begin{aligned}
\left\langle S M_{S}\right| & \tau_{k_{1} q_{1}}^{(b)} \tau_{k_{2} q_{2}}^{(t)}\left|\bar{S} \bar{M}_{S}\right\rangle \\
= & \sum\left\langle S M_{S}\left|\tau_{k_{1} q_{1}}^{(b)}\right| m_{1} m_{2}\right\rangle\left\langle m_{1} m_{2}\left|\tau_{k_{2} q_{2}}^{(t)}\right| \bar{S} \bar{M}_{S}\right\rangle \\
= & \sum\left\langle S M_{S} \mid \frac{1}{2}\left(M_{S}-m_{2}\right), \frac{1}{2} m_{2}\right\rangle\left\langle\bar{S} \bar{M}_{S} \mid \frac{1}{2} m_{1}, \frac{1}{2}\left(\bar{M}_{S}-m_{1}\right)\right\rangle \\
& \times\left\langle\frac{1}{2}\left(M_{S}-m_{2}\right)\left|\tau_{k_{1} q_{1}}^{(b)}\right| m_{1}\right\rangle\left\langle m_{2}\left|\tau_{k_{2} q_{2}}^{(t)}\right| \frac{1}{2}\left(\bar{M}_{S}-m_{1}\right)\right\rangle \\
= & \sum(-)^{q_{1}+q_{2}} \sqrt{\left(2 k_{1}+1\right)\left(2 k_{2}+1\right)} \\
& \times\left\langle S M_{S} \mid \frac{1}{2}\left(M_{S}-m_{2}\right), \frac{1}{2} m_{2}\right\rangle\left\langle\bar{S} \bar{M}_{S} \mid \frac{1}{2} m_{1}, \frac{1}{2}\left(\bar{M}_{S}-m_{1}\right)\right\rangle \\
& \times\left\langle\frac{1}{2}\left(M_{S}-m_{2}\right), k_{1}\left(-q_{1}\right) \mid \frac{1}{2} m_{1}\right\rangle\left\langle\frac{1}{2} m_{2}, k_{2}\left(-q_{2}\right) \mid \frac{1}{2}\left(\bar{M}_{S}-m_{1}\right)\right\rangle
\end{aligned}
$$

It is convenient to couple the remaining spherical harmonics to a common angular momentum and to define

$$
\frac{1}{4 \pi} Y_{\tilde{L} \tilde{M}_{L}}(\hat{p}) Y_{\tilde{l} \tilde{m}_{l}}(\hat{q})=: \sum_{\lambda}\left\langle\tilde{L} \tilde{M}_{L}, \tilde{l}_{\tilde{m}_{l}} \mid \lambda Q\right\rangle B_{\tilde{L} \tilde{l}, \lambda}^{Q}(\hat{q}, \hat{p})
$$

where we used the fact that the sum of the projections turns out to be equal to $q_{1}+q_{2}=Q ; B$ is then

$$
B_{\tilde{L} \tilde{l}, \lambda}^{Q}(\hat{q}, \hat{p})=\sum_{\mu_{L}, \mu_{l}} \frac{1}{4 \pi}\left\langle\tilde{L} \mu_{L}, \tilde{l} \mu_{l} \mid \lambda Q\right\rangle Y_{\tilde{L} \mu_{L}}(\hat{p}) Y_{\tilde{l}_{\mu_{l}}}(\hat{q})
$$

and normalized such that

$$
\int d \Omega_{p} d \Omega_{q} B_{\tilde{L} \tilde{l}, \lambda}^{Q}(\hat{q}, \hat{p})=\delta_{\lambda 0} \delta_{\tilde{L} 0} \delta_{\tilde{l} 0} \delta_{\tilde{Q} 0}
$$

Some properties of $B$ are derived in the next section.

After putting together the individual pieces we arrive at the final result:

$$
\mathcal{T}_{\rho}(\hat{p}, \hat{q})=\frac{1}{4} \sum_{\tilde{L} \tilde{l} \lambda} B_{\tilde{L} \tilde{l}, \lambda}^{Q}(\hat{q}, \hat{p}) \mathcal{A}_{\tilde{L} \tilde{l}, \lambda}^{\rho}
$$

where $\rho=\left\{k_{1} q_{1}, k_{2} q_{2}, k_{3} q_{3}, k_{4} q_{4}\right\}$, and

$$
\mathcal{A}_{\tilde{L} \tilde{l}, \lambda}^{\rho}=\sum_{\alpha, \bar{\alpha}} C_{\tilde{L} \tilde{l}, \lambda}^{\alpha, \bar{\alpha}, \rho} M^{\alpha}\left(M^{\bar{\alpha}}\right)^{\dagger}
$$


with

$$
\begin{aligned}
C_{\tilde{L} \tilde{l}, \lambda}^{\alpha, \bar{\alpha}, \rho}=\frac{1}{4 \pi} & \sum(-)^{M_{S}+M_{S}^{\prime} \kappa} \\
& \times\left\langle S^{\prime} M_{S}^{\prime}, L^{\prime} M_{L}^{\prime} \mid j^{\prime} M_{j}^{\prime}\right\rangle\left\langle j^{\prime} M_{j}^{\prime}, l^{\prime} m_{l}^{\prime} \mid J M_{J}\right\rangle\left\langle S M_{S}, L 0 \mid J M_{J}\right\rangle \\
& \times\left\langle\bar{S}^{\prime} \bar{M}_{S}^{\prime}, \bar{L}^{\prime} \bar{M}_{L}^{\prime} \mid \bar{j}^{\prime} \bar{M}_{j}^{\prime}\right\rangle\left\langle\bar{j}^{\prime} \bar{M}_{j}^{\prime}, \bar{l}^{\prime} \bar{m}_{l}^{\prime} \mid \bar{J} \bar{M}_{J}\right\rangle\left\langle\bar{S} \bar{M}_{S}, \bar{L} 0 \mid \bar{J} \bar{M}_{J}\right\rangle \\
& \times\left\langle L^{\prime} M_{L}^{\prime}, \bar{L}^{\prime}-\bar{M}_{L}^{\prime} \mid \tilde{L} \tilde{M}\right\rangle\left\langle l^{\prime} m_{l}^{\prime}, \bar{l}^{\prime}-\bar{m}_{l}^{\prime} \mid \tilde{l} \tilde{m}\right\rangle\langle\tilde{L} \tilde{M}, \tilde{l} \tilde{m} \mid \lambda Q\rangle \\
& \times\left\langle L^{\prime} 0, \bar{L}^{\prime} 0 \mid \tilde{L} 0\right\rangle\left\langle l^{\prime} 0, \bar{l}^{\prime} 0 \mid \tilde{l} 0\right\rangle \\
& \times\left\langle S M_{S} \mid \frac{1}{2}\left(M_{S}-m_{2}\right), \frac{1}{2} m_{2}\right\rangle\left\langle\bar{S} \bar{M}_{S} \mid \frac{1}{2} m_{1}, \frac{1}{2}\left(\bar{M}_{S}-m_{1}\right)\right\rangle \\
& \times\left\langle\frac{1}{2}\left(m_{1}+q_{1}\right), k_{1}\left(-q_{1}\right) \mid \frac{1}{2} m_{1}\right\rangle\left\langle\frac{1}{2} m_{2}, k_{2}\left(-q_{2}\right) \mid \frac{1}{2}\left(\bar{M}_{S}-m_{1}\right)\right\rangle \\
& \times\left\langle\frac{1}{2}\left(m_{1}^{\prime}-q_{3}\right), k_{3} q_{3} \mid \frac{1}{2} m_{1}^{\prime}\right\rangle\left\langle\frac{1}{2} m_{2}^{\prime}, k_{4} q_{4} \mid \frac{1}{2}\left(\bar{M}_{S}^{\prime}-m_{1}^{\prime}\right)\right\rangle, \quad(\mathrm{C} . g)
\end{aligned}
$$

where the sum runs over $\left\{M_{S}, M_{S}^{\prime}, M_{L}^{\prime}, \bar{M}_{L}^{\prime}, m_{1}, m_{1}^{\prime}\right\}$ and

$$
\kappa=\sqrt{\frac{\left(2 l^{\prime}+1\right)\left(2 \bar{l}^{\prime}+1\right)(2 L+1)(2 \bar{L}+1)\left(2 \bar{L}^{\prime}+1\right)\left(2 L^{\prime}+1\right)\left(2 k_{1}+1\right)\left(2 k_{2}+1\right)}{(2 \tilde{L}+1)(2 \tilde{l}+1)(2 J+1)(2 \bar{J}+1)}} .
$$

\section{On the non-factorization of a strong final state interaction}

In this appendix we will demonstrate the need for a consistent treatment of both the $N N$ scattering and production amplitudes in order to obtain quantitative predictions of meson-production reactions.

Let us assume a separable $N N$ potential

$$
V\left(p^{\prime}, k\right)=\alpha g\left(p^{\prime}\right) g(k)
$$

where $\alpha$ is a coupling constant and $g(p)$ an arbitrary real function of $p$. With this potential the $T$-matrix scattering equation can be readily solved to yield

$$
T\left(p^{\prime}, k\right)=\frac{V\left(p^{\prime}, k\right)}{1-R\left(p^{\prime}\right)+i \kappa\left(p^{\prime}\right) V\left(p^{\prime}, p^{\prime}\right)},
$$

with

$$
R\left(p^{\prime}\right) \equiv m \mathbf{P} \int_{0}^{\infty} d k^{\prime} \frac{k^{\prime 2} V\left(k^{\prime}, k^{\prime}\right)}{p^{\prime 2}-k^{\prime 2}}
$$

and $\kappa(p)=p \pi \mu$ denotes the phase space density here expressed in terms of the reduced mass of the outgoing two nucleon pair $\mu=m_{N} / 2$. 
Note that for an arbitrary function $g(k)$, such as $g(k) \equiv 1$ as discussed below, $R\left(p^{\prime}\right)$ may be divergent. In this case $R$ is to be understood as properly regularized. The principal value integral $R\left(p^{\prime}\right)$ given above is therefore a model-dependent quantity, for it depends on the regularization scheme used. The condition that the on-shell $N N$ scattering amplitude should satisfy Eq. 6 relates this to the on-shell potential, $V\left(p^{\prime}, p^{\prime}\right)$ :

$$
R\left(p^{\prime}\right)=1+\kappa\left(p^{\prime}\right) \cot \left(\delta\left(p^{\prime}\right)\right) V\left(p^{\prime}, p^{\prime}\right)
$$

where it is assumed that $\eta\left(p^{\prime}\right)=1$. This shows that, for a given potential, the regularization should be such that Eq. (D.4) be satisfied in order to reproduce Eq. 6. Indeed, in conventional calculations based on meson exchange models, where one introduces form factors to regularize the principal value integral, the cutoff parameters in these form factors are adjusted to reproduce the $N N$ scattering phase shifts through Eq. 6. Conversely, for a given regularization scheme, the $N N$ potential should be adjusted such as to obey Eq. (D.4). This is the procedure used in effective field theories [311], where the coupling constants in the $N N$ potential are dependent on the regularization.

We also assume that the production amplitude $M$ is given by a separable form,

$$
M(E, k)=\beta g(k) h(p),
$$

where $\beta$ is a coupling constant and $h(p)$ an arbitrary function of the relative momentum $p$ of the two nucleons in the initial state. With this we can express the total transition amplitude as

$$
A\left(E, p^{\prime}\right)=-\frac{1}{\kappa\left(p^{\prime}\right)} e^{i \delta\left(p^{\prime}\right)} \sin \left(\delta\left(p^{\prime}\right)\right)\left(\frac{M\left(E, p^{\prime}\right)}{V\left(p^{\prime}, p^{\prime}\right)}\right) .
$$

Equation D.6 is the desired formula for our discussion. It allows us to study the relationship between the $N N$ potential and the production amplitude $M\left(E, p^{\prime}\right)$ explicitly as different regularization schemes are used. For this purpose let us study the simplest case of a contact $N N$ potential (setting the function $g=1$ ) in the limit $p^{\prime} \rightarrow 0$. If we regularize the integrals by means of the power divergent subtraction (PDS) scheme [311] we get

$$
R=-\frac{a \mu}{1-a \mu}
$$


where $\mu$ denotes the regularization scale. Substituting this result into Eq. (D.4), we obtain

$$
\alpha=\left(\frac{2 a}{\pi m}\right) \frac{1}{1-a \mu}
$$

for the $N N$ coupling strength. Note that for $\mu=0$ the PDS scheme reduces to that of minimal subtraction [311]. Since the total production amplitude $A$ should not depend on the regularization scale we immediately read off Eq. D.6 that

$$
\beta \propto(1-a \mu)^{-1}
$$

Therefore the model clearly exhibits the point made in section 2: Namely, the necessity of calculating both the production amplitude and the FSI consistently in order to allow for quantitative predictions.

\section{E Chiral Counting for Pedestrians}

In this appendix we demonstrate how to estimate the size of a particular loop integral. This is a necessary step in identifying the chiral order of a diagram. It should be clear, however, that the same methods can be used to estimate the size of any integral. However, the importance of the chiral symmetry is that it ensures the existence of an ordering scheme that suppresses higher loops.

The necessary input are the expressions for the vertices and propagators at any given order. For the chiral perturbation theory those can be found in Ref. [197]. In addition we need an estimate for the measure of the integral.

Once each piece of a diagram is expressed in terms of the typical momenta/energies, one gets an estimate of the value of the particular diagram. The procedure works within both time-ordered perturbation theory and covariant theory. Obviously, for each irreducible diagram both methods have to give the same answer. If a diagram has a pure two-nucleon intermediate state, as is the case for the direct production, the covariant counting can only give the leading order piece of the counting within TOPT.

In this appendix we study only diagrams that are three-particle irreducible; i.e., the topology of the diagram does not allow an intermediate two-nucleon 


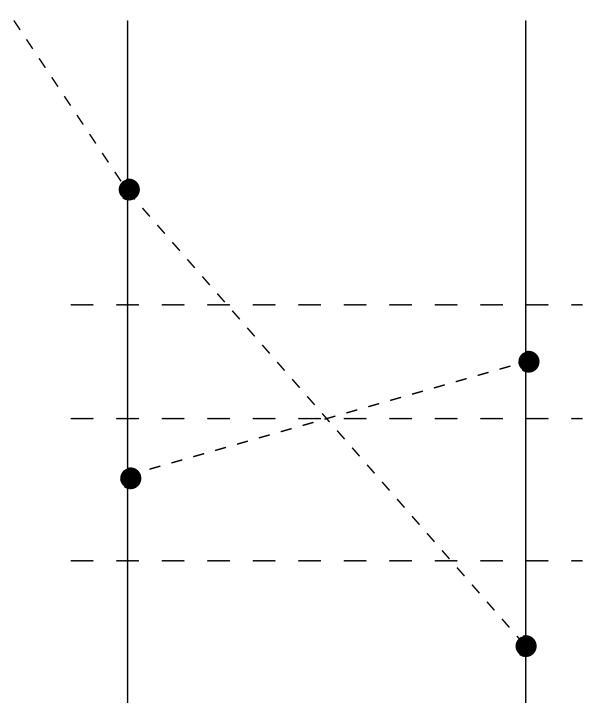

Fig. E.1. A typical loop that contributes to pion production in nucleon-nucleon collisions. Solid lines denote nucleons and dashed lines pions. The solid dots show the points of interactions. The horizontal dashed lines indicate equal time slices, as needed for the evaluation of the diagram in time-ordered perturbation theory.

state to go on-shell. The reducible diagrams require a different treatment and are discussed in detail in the main text. There is another group of diagrams mentioned in the main text that is not covered by the counting rules presented, namely radiation pions. Those occur if a pion in an intermediate state goes on-shell. It is argued in the main text that these are suppressed, because the pion, in order to go on-shell, is only allowed to carry momenta of the order of the external momenta, and thus the momentum scale within the loop is of order of the pion mass and not of the order of the initial momentum. Therefore we do not consider radiation pions any further.

\section{E.1 Counting within TOPT}

As mentioned above, if we want to assign a chiral order to a diagram, all we need to do is to replace each piece in the complete expression for the evaluation of the matrix element by its value when all momenta are of their typical size. In case of meson production in nucleon-nucleon collisions this typical momentum is given by the initial momentum $p_{i}$. Time-ordered perturbation theory contains only three-dimensional integrals and thus we do not need to 
fix the energy scale in the integral.

The counting rules are

- the energy of virtual pions is interpreted as $\mathcal{O}\left(p_{i}\right)^{42}$ and thus

- every time slice that contains a virtual pion is interpreted as $1 / p_{i}$ (see Fig.

- for each virtual pion line put an additional $1 / p_{i}$ (from the vertex factors),

- interpret the momenta in the vertices as $p_{i}$

- every time slice that contains no virtual pion is interpreted as $1 / m_{\pi} ;$ most of these diagrams, however, are reducible (c.f. main text)

- the integral measure is taken as $p_{i}^{3} /(4 \pi)^{2}$.

Here we used that $p_{i}^{2} / M_{N} \simeq m_{\pi} \ll p_{i}$, in accordance with Eqs. (80), and thus nucleons can be treated as static in the propagators if there is an additional pion present. However if there is a time slice that contains two nucleons only, the corresponding propagator needs to be identified with the inverse of the typical nucleon energy $1 / m_{\pi}$ and the static approximation is very bad [155].

In the diagram of Fig. E.1 three $\pi N N$ vertices, each $\simeq p_{i} / f_{\pi}$, appear as well as the $\pi \pi N N$ Weinberg-Tomozawa vertex $\simeq p_{i} / f_{\pi}^{2}$. In addition the three time slices give a factor $1 / p_{i}^{3}$ and we also need to include a factor $1 / p_{i}^{2}$, since there are two virtual pions. We therefore find

$$
M^{T O P T} \simeq\left(\frac{p_{i}}{f_{\pi}}\right)^{3} \frac{p_{i}}{f_{\pi}^{2}}\left(\frac{1}{p_{i}}\right)^{5} \frac{p_{i}^{3}}{(4 \pi)^{2}} \simeq \frac{1}{f_{\pi}^{3}}\left(\frac{m_{\pi}}{M_{N}}\right) .
$$

Here we used $4 \pi f_{\pi} \simeq M_{N}$ and $p_{i} \simeq \sqrt{M_{N} m_{\pi}}$.

\section{E.2 Counting within the covariant scheme}

Naturally, as TOPT and the covariant scheme are equivalent, the chiral order that is to be assigned to some diagram needs to be the same in both schemes. The reason why we demonstrate both is that here we are faced with a problem

$\overline{42}$ There is one exception to this rule: if a time derivative acts on a pion on a vertex, where all other particles are on-shell, then energy conservation fixes the energy. 
in which the typical energy scale $m_{\pi}$ and the typical momentum scale $p_{i}$ are different. In the covariant approach a four-dimensional integral measure enters and naturally the question arises whether $m_{\pi}$ or $p_{i}$ is appropriate for the zeroth component of this measure $p_{0}$. For example, in Ref. [163] it was argued, that one should choose $m_{\pi}$, although this choice is by no means obvious from the structure of the integrals. However, given the experience we now have in dealing with loops in time--ordered perturbation theory, where these ambiguities do not occur, the answer is simple: we just have to assign that scale to $p_{0}$ that will reproduce the same order for any diagram as in the counting within TOPT [204]. Once the choice for $p_{0}$ is fixed, the much easier to use covariant counting can be used to estimate the size of any loop integral. Thus have the following rules:

- the energy of virtual pions is interpreted as $\mathcal{O}\left(p_{i}\right)^{43}$

- each pion propagator is taken as $\mathcal{O}\left(1 / p_{i}^{2}\right)$

- each nucleon propagator that cannot occur in a two-nucleon cut is taken as $\mathcal{O}\left(1 / p_{0}\right)$ (the leading contribution of a nucleon propagator that can occur in a two-nucleon cut is $\mathcal{O}\left(1 / m_{\pi}\right)$; most of these diagrams, however, are reducible (c.f. main text))

- interpret the momenta in the vertices as $p_{i}$

- the integral measure is taken as $p_{0} p_{i}^{3} /(4 \pi)^{2}$ (when the diagram allows for a two-nucleon cut the measure reads $\left.\left(m_{\pi} p_{i}^{3}\right) /(4 \pi)^{2}\right)$.

Thus we have for the diagram of Fig. E.1

$$
M^{\operatorname{cov}} \simeq\left(\frac{p_{i}}{f_{\pi}}\right)^{3} \frac{p_{i}}{f_{\pi}^{2}}\left(\frac{1}{p_{i}^{2}}\right)^{2}\left(\frac{1}{p_{0}}\right)^{2} \frac{p_{0} p_{i}^{3}}{(4 \pi)^{2}} \simeq \frac{1}{f_{\pi}^{3}}\left(\frac{m_{\pi}}{M_{N}}\right)\left(\frac{p_{i}}{p_{0}}\right)
$$

Thus, we need to assign $p_{0} \sim p_{i}$ in order to get the same result in both schemes. As a side result we also showed, that the nucleons are indeed static in leading order inside loops that do not have a two-nucleon cut, as pointed out in Ref. [206].

\footnotetext{
$\overline{{ }^{43} \text { With }}$ the same exception as in the time ordered situation (c.f. corresponding footnote).
} 


\section{List of Figures}

1 Comarison of two different reactions that can be used to extract parameters of a strong final state interaction. Shown is a typical low momentum transfer reaction (diagram a), e.g., $\left.K^{-} d \rightarrow \gamma \Lambda n\right)$ and a large momentum transfer reaction (diagram b), e.g., $p p \rightarrow p K^{+} \Lambda$ ).

2 Some possible contributions to the production operator for neutral pion production in pp collisions. Solid lines are nucleons, dashed lines are pions.

3 Sketch of the production reaction showing the initial state interaction amongst the two nucleons (ISI), the final state interaction of all outgoing particles (FSI) as well as the production operator $A$ as defined in the text. The left diagram indicates the complexity possible, whereas the right diagram shows the first and potentially leading term for the FSI only.

$4 \quad$ Illustration of the momentum mismatch. The picture shows why one should a priori expect meson exchange currents to be very important close to the threshold.

5 Diagrammatic presentation of the distorted wave Born approximation.

6 Energy dependence of two different spin cross sections for the reaction $p p \rightarrow p p \pi^{0}$. The data for ${ }^{1} \sigma_{0}\left({ }^{3} \sigma_{1}\right)$, shown as solid (opaque) dots, are compared to a fit of the form $y=A \eta^{\tau}$ with $\tau=6(\tau=8)$. The data are from table $V$ of Ref. [47] with an additional error of $10 \%$ on the total cross section. 
$7 \quad$ Illustration of the sources of a discontinuity in the amplitude A from two baryon intermediate states. The dashed horizontal lines indicate the presence of a two baryon unitarity cut (Diagrams with initial state interaction are not shown explicitly (c.f. Fig. 5)).

8 The integration contours in the complex $m^{\prime 2}$ plane to be used in the original treatment by Geshkenbein [55,56] (a) and in the derivation of Eq. (18) (b). The thick lines indicate the branch cut singularities.

9 Illustration of the choice of variables in the over all center-of-mass system.

10 Sketch of a Dalitz plot for a reaction with a three-body final state (particles are labeled as 1,2 and 3). Regions of possibly strong final state interactions in the $(i j)$ system are labeled by $F_{(i j)}$; a resonance in the (12)-system would show up like the band labeled as $R$. The region of a possible interference of the two is labeled as I.

11 Angular distributions for the reaction $p p \rightarrow d \bar{K}^{0} K^{+}$measured at $Q=46 \mathrm{MeV}$ [66]. The solid line shows the result of the overall fit including both $\bar{K} K$ s-waves as well as $p$-waves. To obtain the dashed (dotted) line, the parameters for the $p$-wave (s-wave) were set to zero (see text). The small error shows the statistical uncertainty only, whereas the large ones contain both the systematic as well as the statistical uncertainty added linearly.

12 Various mass distributions for the reaction $p p \rightarrow d \bar{K}^{0} K^{+}$ (Line code as in Fig. 11). The small error bars show the statistical uncertainty only, whereas the large ones contain both the systematic as well as the statistical uncertainty (c.f. Ref. [66]). 
13 Some polarization observables reported in Ref. [47] for the reaction $\vec{p} \vec{p} \rightarrow p p \pi^{0}$ at $\eta=0.83$ as a function of the pion angle compared to predictions of the model of Ref. [71]. The solid lines show the results for the full model whereas contributions from the Delta where omitted for the dashed lines.

14 Some polarization observables reported in Ref. [47] for the reaction $\overrightarrow{p p} \rightarrow p p \pi^{0}$ at $\eta=0.83$ as a function of $\cos \left(\theta_{p}\right)$ compared to predictions of the model of Ref. [71]. The solid lines show the results for the full model whereas contributions from the Delta were omitted for the dashed lines.

15 Sensitivity of the analyzing power as well as the differential cross section for the reaction $p n \rightarrow p p \pi^{-}$to the sign of the ${ }^{3} P_{0} \rightarrow{ }^{1} S_{0} s$ amplitude $a_{1}$. The lines corresponds to the model of Ref. [73]: the solid line is the model prediction whereas for the dashed line the sign of $a_{1}$ was reversed. The experimental data are from Ref. [74] and [75] at $T_{L a b}=353 \mathrm{MeV}(\eta=0.65)$.

16 Illustration of the coordinate system used in the analysis for the reaction $p p \rightarrow d \bar{K}^{0} K^{+}$.

17 Demonstration of the selectivity of the spin cross sections. Shown are the spectra of the reaction $\vec{p} \vec{p} \rightarrow p n \pi^{+}$as a function of $\epsilon$ for an excess energy of $25 \mathrm{MeV}$. In each panel the solid line shows the full result for the corresponding cross section, the dot-dashed, long-dashed, dashed, and dotted lines show the $S p, S s, S d$, and Pp contribution respectively. The curves are from the model of Ref. [71].

18 The lowest meson production thresholds for single meson production in proton-proton collisions together with the corresponding energy ranges of the modern cooler synchrotrons. 
19 Illustration of different sources of charge symmetry breaking: diagram a) shows CSB in the production operator through $\pi-\eta$ mixing and diagram b) shows CSB in the propagation of the scalars. Thin solid lines denote nucleons, thick solid lines scalar mesons and dashed ones pseudoscalar mesons. The $X$ indicates the occurrence of a CSB matrix element.

20 Comparison of the model predictions of Ref. [73] to the data. The references for the experimental data can be found in table 8. The solid lines show the results of the full model; the dashed line shows the results without the $\Delta$ contributions.

21 The NN phase shifts for the model of Ref. [175] in the energy range relevant for pion production. The pion production threshold is at $E_{L A B}=286 \mathrm{MeV}$. The experimental data are from Refs. [17r]] (triangles) and [61] (circles).

22 Contributions to the potential of the model of Ref. [179].

23 The $\pi N$ phase shifts for the model of Ref. [179] in the energy range relevant for pion production. The experimental data are from Refs. [183,184]. Note the different scales of the various panels.

24 Comparison of the model predictions to the data taken from Ref. [47] (pp $\left.\rightarrow p p \pi^{o}\right)$, Ref. [108] $\left(p p \rightarrow d \pi^{+}\right)$and Ref. [94] $\left(p p \rightarrow p n \pi^{+}\right)$.

26 Illustration of the role of the $4 N \pi$ contact term in $N N \rightarrow N N \pi$ and three nucleon scattering. Solid lines denote nucleons, dashed lines denote pions. 
27 Comparison of the predictions from effective field theory at $L O$ (dashed line) and $N L O$ (with the $\pi N$ parameters from a $N L O$ (dotted line) and an NNLO (solid line) analysis) with the ${ }^{3} \sigma_{1}$ cross section for $p p \rightarrow p p \pi^{0} \quad[47]$.

$28 a_{0}$ of $p p \rightarrow n p \pi^{+}$in chiral perturbation theory. The different lines correspond to values of the parameter related to the three-nucleon force: $\delta=1$ (long dashed line). $\delta=0$ (dot-dashed line), $\delta=-0.2$ (solid line), and $\delta=-1$ (short dashed line). Data are from Ref. [219].

29 Visualisation of Eq. (90).

30 The one sigma exchange as it is perturbatively built up in the effective field theory, starting from the left with the lowest order diagram (NLO). The chiral order increases by one power in $\chi$ between each diagram from the left to the right.

31 Leading cut structure of the one pion exchange potential in nucleon-nucleon scattering.

32 Results for the total cross sections of the reactions $p p \rightarrow p p \eta$, $p n \rightarrow p n \eta$, and $p n \rightarrow d \eta$ from Ref. [34] employing different $N N$ models for the final state interaction. The solid lines represent the results with the CCF NN model [175] whereas the dashed-dotted lines were obtained for the Bonn B model [25]. Data are from Refs. [33,109-112,120].

33 Invariant mass spectrum for the two-proton system for the reaction $p p \rightarrow p p \eta$ at $Q=15 \mathrm{MeV}$. The dashed line shows the Ss contribution, the dot-dashed line the Ps distribution and the solid line the incoherent sum of both. The data is taken from Ref. [113]. 
34 Comparison of the quality of available data for the reactions $p \Lambda$ elastic scattering (data are from [264-266]) and $p p \rightarrow K^{+} \Lambda p$ at $T_{\text {Lab }}=2.3 \mathrm{GeV}[267]^{44}$. In both panels the red curve corresponds to a best fit to the data. In the left panel the dashed lines represent the spread in the energy behavior allowed by the data, according to the analysis of Ref. [266]; analogous curves in the right panel would lie almost on top of the solid line and are thus not shown explicitly.

35 Values allowed for the spin singlet and spin triplet scattering length by the $\Lambda N$ elastic scattering data according to Ref. [266]. The dark shaded area denotes the $1 \sigma$ range for the parameters and the light shaded area the $2 \sigma$ range. The cross shows the best fit value $\left(a_{s}=1.8 \mathrm{fm}\right.$ and $\left.a_{t}=1.6 \mathrm{fm}\right)$.

36 Graphical illustration of the leading contribution to the $f_{0}-a_{0}$ mixing matrix element $\Lambda$ defined in Eq. (91).

37 Modulus of the leading piece of the mixing amplitude $\Lambda$ defined in Eq. (91). The two kinks occur at the $K^{+} K^{-}$(at 987.35 $\mathrm{MeV})$ and the $\bar{K}^{0} K^{0}(995.34 \mathrm{MeV})$ threshold respectively.

38 Possible diagrams to contribute to the reaction $N d \rightarrow 3 N x$. In diagram a) and b) the three nucleons in the final state from a bound state, whereas diagram c) shows the dominant diagram in quasi-free kinematics. Here it is assumed that only a particular nucleon pair (to be identified through proper choice of kinematics) interacts in the final state.

\footnotetext{
${ }_{44}$ The data was taken inclusively (only the kaon was measured in the final state), however, for the small invariant masses shown only the $\Lambda \mathrm{K}$ channel is open.
} 
39 Momentum distribution of proton-spectators in the reaction $\bar{p} d \rightarrow 3 \pi^{+} 2 \pi^{-} p$. The data are taken from Ref. [305] while the solid line is is based on a calculation that includes the quasi-free production as well as meson rescattering. The figure is taken from [306], where also details about the corresponding calculation can be found.

E.1 A typical loop that contributes to pion production in nucleonnucleon collisions. Solid lines denote nucleons and dashed lines pions. The solid dots show the points of interactions. The horizontal dashed lines indicate equal time slices, as needed for the evaluation of the diagram in time-ordered perturbation theory.

\section{List of Tables}

1 The lowest partial waves for the production of a pseudo-scalar particle in $N N \rightarrow N N x$.

2 The lowest partial waves for the production of a scalar particle in $N N \rightarrow N N x$.

3 Results for the $C$ parameters from a fit to the experimental data. The parameters are given in units of $C_{0}^{q^{\prime}}$.

4 Relations between spherical tensors and some observables that do not contain the final state polarization following Ref. [69]. To simplify notation, the indices specifying the final state polarization are dropped. The symbol * indicates a possible set of independent observables. Note: For pp induced reactions more observables become equivalent, as described in the text. Those are marked by $\left(^{*}\right)$. The linear combinations of spin correlation observables appearing in the table are defined in Eqs. (39). 
$5 \quad$ Relations between spherical tensors and some observables that do contain the final state polarization. The symbol * indicates a possible set of independent observables. The linear combinations of spin correlation observables appearing in the table are defined in Eqs. (40).

6 List of a possible set of the independent amplitude structures that contribute to the reaction $p p \rightarrow p p+($ pseudoscalar $)$. In the last column shows the lowest partial waves for the final state that contribute to the given amplitude (using the notation of section 1.6). To keep the expressions simple we omitted to give the symmetric, trace free expressions for the terms listed in the last two lines.

$7 \quad$ List of the lowest partial waves in the final state that contribute to the individual spin cross sections. Capital letters denote the baryon-baryon partial waves whereas small letters that of the meson with respect to the baryon-baryon system.

8 List of observables measured for various $N N \rightarrow N N x$ channels for excess energies up to $Q=40 \mathrm{MeV}$.

9 The various low energy constants $c_{i}$ in units of $G e V^{-1}$. The first two columns give the values from the original references (left column: tree level calculation of Ref. [171]; right column: one loop calculation of Ref. [172]), whereas the last two columns give those values reduced by the Delta contribution as described in the text. It is those numbers that where used in the calculations presented here.

10 Comparison of the corresponding chiral order in the Weinberg scheme $\left(p \sim m_{\pi}\right)$ and the new counting scheme $\left(p \sim \sqrt{m_{\pi} M_{N}}\right)$ for several nucleonic contributions for $p$-wave pion production. Subleading vertices are marked as $\odot$. Here $q$ denotes the external pion momentum. For simplicity we assume the outgoing nucleons at rest. 
11 Comparison of the corresponding chiral order in the Weinberg scheme $\left(p \sim m_{\pi}\right)$ and the new counting scheme $\left(p \sim \sqrt{m_{\pi} M_{N}}\right)$ for several nucleonic contributions for $s$-wave pion production. Subleading vertices are marked as $\odot$. Not shown explicitly are the recoil corrections for low-order diagrams. For example, recoil corrections to diagram b) appear at order $\mathrm{pm}_{\pi} / M_{N}^{2}$.

12 Comparison of the corresponding chiral order in the Weinberg scheme $\left(p \sim m_{\pi}\right)$ and the new counting scheme $\left(p \sim \sqrt{m_{\pi} M_{N}}\right)$ for the leading and next-to-leading $\Delta$ contributions for s-wave pion production. Subleading vertices are marked as $\odot$. Not shown explicitly are the recoil corrections for low-order diagrams.

13 Comparison of the results of the analytic calculation of Ref. [205] with the expectations based on the two counting schemes as discussed in the text. The diagrams are labeled as in Fig. 11 (the label $j_{R}$ shows that here the recoil term of diagram $j$ is calculated; it appears at NNLO in the Weinberg scheme as well as in the new counting scheme).

A.1 The values for $T_{L a b}, Q$ and $\eta$ for the different channels for pion production.

\section{References}

[1] H. Machner and J. Haidenbauer. J. Phys., G25:R231-R271, 1999.

[2] P. Moskal, M. Wolke, A. Khoukaz, and W. Oelert. Prog. Part. Nucl. Phys., 49:1, 2002.

[3] S. Hirenzaki, P. Fernandez de Cordoba, and E. Oset. Phys. Rev., C53:277-284, 1996.

[4] H. P. Morsch et al. Phys. Rev. Lett., 69:1336-1339, 1992.

[5] G. A. Miller, B. M. K. Nefkens, and I. Slaus. Phys. Rept., 194:1-116, 1990. 
[6] A. K. Opper et al. nucl-ex/0306027, 2003.

[7] V. Baru, J. Haidenbauer, C. Hanhart, and J. A. Niskanen. nucl-th/0303061, 2003.

[8] W. R. Gibbs, B. F. Gibson, and Jr. Stephenson, G. J. Phys. Rev., C16:327, 1977.

[9] W. R. Gibbs, S. A. Coon, H. K. Han, and B. F. Gibson. Phys. Rev., C61:064003, 2000.

[10] H. Garcilazo and T. Mizutani. $\pi N N$ Systems. World Scientific, Singapore, 1990.

[11] B. Blankleider and I. R. Afnan. Phys. Rev., C24:1572-1595, 1981.

[12] Y. Avishai and T. Mizutani. Nucl. Phys., A326:352, 1979.

[13] A. W. Thomas and A. S. Rinat. Phys. Rev., C20:216-224, 1979.

[14] B. Blankleider and A. N. Kvinikhidze. Few Body Syst. Suppl., 12:223-228, 2000.

[15] P. U. Sauer, M. Sawicki, and S. Furui. Prog. Theor. Phys., 74:1290, 1985.

[16] B. Blankleider and A. N. Kvinikhidze. Few Body Syst. Suppl., 7:294-308, 1994.

[17] B. K. Jennings. Phys. Lett. B, 205:187, 1988.

[18] G. H. Lamot, J. L. Perrot, C. Fayard, and T. Mizutani. Phys. Rev., C35:239$253,1987$.

[19] A. N. Kvinikhidze and B. Blankleider. Nucl. Phys., A574:788, 1994.

[20] D. R. Phillips and I. R. Afnan. Ann. Phys., 240:266, 1995.

[21] A. D. Lahiff and I. R. Afnan. Phys. Rev., C60:024608, 1999.

[22] E. van Faassen and J. A. Tjon. Phys. Rev. C, 33:2105, 1986.

[23] A. N. Kvinikhidze and B. Blankleider. Phys. Lett., B307:7-12, 1993.

[24] Ch. Elster, K. Holinde, D. Schütte, and R. Machleidt. Phys. Rev. C, 38:1828, 1988.

[25] R. Machleidt. Adv. Nucl. Phys., 19:189, 1989. 
[26] R. Machleidt, K. Holinde, and Ch. Elster. Phys. Rep., 149:1, 1987.

[27] A. H. Rosenfeld. Phys. Rev., 96:1, 1954.

[28] M. Gell-Mann and K. M. Watson. Annu. Rev. Nucl. Sci., 4:219, 1954.

[29] P. Moskal et al. Phys. Rev. Lett., 80:3202-3205, 1998.

[30] P. Moskal et al. Phys. Lett., B482:356-362, 2000.

[31] D. Koltun and A. Reitan. Phys. Rev., 141:1413, 1966.

[32] A. Gasparyan, J. Haidenbauer, C. Hanhart, and J. Speth. arXiv:hepph/0311116.

[33] H. Calén et al. Phys. Rev. C, 58:2667, 1998.

[34] V. Baru et al. Phys. Rev., C67:024002, 2003.

[35] K. Nakayama, J. Speth, and T. S. H. Lee. Phys. Rev., C65:045210, 2002.

[36] G. Faldt and C. Wilkin. Phys. Scripta, 64:427-438, 2001.

[37] M. Goldberger and K.M. Watson. Collision Theory. Wilney, New York, 1964.

[38] J. Gillespie. Final-State interactions. Holden-Day, INC., San Francisco, 1964.

[39] A. Sibirtsev and W. Cassing. nucl-th/9904046, 1999.

[40] C. Hanhart and K. Nakayama. Phys. Lett., B454:176-180, 1999.

[41] K. Watson. Phys. Rev., 88:1163, 1952.

[42] C. J. Joachin. Quantum Collision Theory. North-Holland, Amsterdam, 1975.

[43] H. O. Meyer et al. Phys. Rev. Lett., 65:2846, 1990.

[44] H. O. Meyer et al. Nucl. Phys., A539:633, 1992.

[45] G. Miller and P. Sauer. Phys. Rev. C, 44:1725, 1991.

[46] J. T. Balewski et al. Eur. Phys. J., A2:99-104, 1998.

[47] H. O. Meyer et al. Phys. Rev., C63:064002, 2001.

[48] J. W. de Maag, L. P. Kok, and H. van Haeringen. J. Math. Phys., 25:684, 1984.

[49] F.M. Renard and Y. Renard. Nucl. Phys., B1:389, 1967. 
[50] B. Mecking et al. CEBAF proposal PR-89-045 (1989).

[51] R. A. Adelseck and L. E. Wright. Phys. Rev., C39:580-586, 1989.

[52] X. Li and L. E. Wright. J. Phys., G17:1127-1137, 1991.

[53] H. Yamamura, K. Miyagawa, T. Mart, C. Bennhold, and W. Glockle. Phys. Rev., C61:014001, 2000.

[54] B. O. Kerbikov. Phys. Atom. Nucl., 64:1835-1840, 2001.

[55] B. V. Geshkenbein. Sov. J. Nucl. Phys., 9:720, 1969.

[56] B. V. Geshkenbein. Phys. Rev., D61:033009, 2000.

[57] W. Eyrich et al. A. Sibirtsev. in preparation.

[58] R.D. Spital T.H. Bauer and D.R. Yennie. Rev. Mod. Phys., 50:261, 1978.

[59] K. Tsushima and K. Nakayama. Phys. Rev., C68:034612, 2003.

[60] M. Altmeier et al. Phys. Rev. Lett., 85:1819-1822, 2000.

[61] R. A. Arndt, I. Strakovsky, and R. Workman. Phys. Rev. C, 50:731, 1994.

[62] F. Rathmann. in Hadron physics at COSY, A. Gillitzer et al. (eds.), arXiv:hep$\mathrm{ph} / 0311071$.

[63] Eberhard Klempt. hep-ex/0101031, 2000.

[64] E. Byckling and K. Kajantie. Particle Kinematics. John Wiley and Sons, New-York, 1973.

[65] K. Kilian. private communication.

[66] V. Kleber et al. nucl-ex/0304020, 2003.

[67] A. E. Kudryavtsev, V. E. Tarasov, J. Haidenbauer, C. Hanhart, and J. Speth. Phys. Rev., C66:015207, 2002.

[68] G. G. Ohlsen. Rep. Prog. Phys., 35:717-801, 1972.

[69] L.D. Knutson. AIP Conf. Proc., 512:177-192, 2000.

[70] B. Bassalleck et al. Phys. Rev. Lett., 89:212302, 2002.

[71] C. Hanhart, J. Haidenbauer, O. Krehl, and J. Speth. Phys. Rev., C61:064008, 2000 . 
[72] M. P. Rekalo and E. Tomasi-Gustafsson. nucl-th/0105002, 2001.

[73] C. Hanhart, J. Haidenbauer, O. Krehl, and J. Speth. Phys. Lett., B444:25-31, 1998.

[74] H. Hahn et al. Phys. Rev. Lett., 82:2258-2261, 1999.

[75] F. Duncan et al. Phys. Rev. Lett., 80:4390-4393, 1998.

[76] N. K. Pak and M. P. Rekalo. Eur. Phys. J., A12:121-124, 2001.

[77] M. Daum et al. Eur. Phys. J., C23:43-59, 2002.

[78] M. Daum et al. Eur. Phys. J., C25:55-65, 2002.

[79] Y. Maeda, N. Matsuoka, and K. Tamura. Nucl. Phys., A684:392-396, 2001.

[80] S.E. Vigdor. Flavour and Spin in Hadronic and Electromagnetic Interactions, ed. F. Balestra et al. (Italian Physical Society, Bologna, 1993), p. 317.

[81] E. Oset, Jose A. Oller, and U.-G. Meißner. Eur. Phys. J., A12:435-446, 2001.

[82] C. Hanhart. nucl-th/0306073, 2003.

[83] S.M. Bilenky and R.M. Ryndin. Phys. Lett., 6:217, 1963.

[84] P. Thorngren Engblom et al. Nucl. Phys., A663:447-451, 2000.

[85] P. N. Deepak and G. Ramachandran. Phys. Rev., C65:027601, 2002.

[86] A. Bondar et al. Phys. Lett. B, 356:8, 1995.

[87] R. Bilger et al. Nucl. Phys., A693:633-662, 2001.

[88] S. AbdEl Samad et al. nucl-ex/0212024, 2002.

[89] S. Stanislaus, D. Horvath, D. F. Measday, and A. J. Noble. Phys. Rev. C, 44:2287, 1991.

[90] G. Rappenecker et al. Nucl. Phys., A590:763, 1995.

[91] W. W. Daehnick et al. Phys. Rev. Lett., 74:2913, 1995.

[92] J. G. Hardie et al. Phys. Rev. C, 59:20, 1997.

[93] K.T. Brinkmann et al. Acta Physica Polonica B, 29:2993, 1998.

[94] W. W. Daehnick et al. Phys. Rev., C65:024003, 2002. 
[95] D. A. Hutcheon et al. Phys. Rev. Lett., 64:176, 1990.

[96] C. M. Rose. Phys. Rev., 154:1305, 1967.

[97] B. G. Ritchie et al. Phys. Rev. Lett., 66:568, 1991.

[98] D. Aebischer et al. Nucl. Phys., B108:214, 1978.

[99] F. Shimizu et al. Nucl. Phys., A386:571, 1982.

[100] D. Axen et al. Nucl. Phys., A256:387, 1976.

[101] B. M. Preedom et al. Phys. Lett. B, 65:31, 1976.

[102] B. G. Ritchie et al. Phys. Lett. B, 300:24, 1993.

[103] M. Drochner et al. Phys. Rev. Lett., 77:454, 1996.

[104] P. Heimberg et al. Phys. Rev. Lett., 77:1012, 1996.

[105] E. Korkmaz et al. Nucl. Phys., A535:637-650, 1991.

[106] E. L. Mathie et al. Nucl. Phys., A397:469, 1983.

[107] C. L. Dolnick. Nucl. Phys., B22:461, 1970.

[108] B. von Przewoski et al. Phys. Rev., C61:064604, 2000.

[109] H. Calén et al. Phys. Rev. B, 366:366, 1996.

[110] H. Calén et al. Phys. Rev. Lett., 79:2642, 1997.

[111] J. Smyrski et al. Phys. Lett. B, 474:182, 2000.

[112] P. Moskal et al. nucl-ex/0307005, 2003.

[113] M. Abdel-Bary et al. Eur. Phys. J., A16:127-137, 2003.

[114] H. Calen et al. Phys. Rev. Lett., 79:2642-2645, 1997.

[115] A. M. Bergdolt et al. Phys. Rev., D48:2969-2973, 1993.

[116] E. Chiavassa et al. Phys. Lett., B322:270-274, 1994.

[117] F. Hibou et al. Phys. Lett., B438:41-46, 1998.

[118] H. Calen et al. Phys. Lett., B458:190-196, 1999.

[119] P. Winter et al. Phys. Lett., B544:251-258, 2002. 
[120] H. Calén et al. Phys. Rev. Lett., 80:2069, 1998.

[121] P. Moskal et al. Phys. Lett., B474:416-422, 2000.

[122] J. T. Balewski et al. Phys. Lett. B, 388:859, 1996.

[123] J. T. Balewski et al. Phys. Lett., B420:211-216, 1998.

[124] S. Sewerin et al. Phys. Rev. Lett., 83:682-685, 1999.

[125] F. Hibou et al. Phys. Rev. Lett., 83:492-495, 1999.

[126] S. Barsov et al. nucl-ex/0305031, 2003.

[127] F. Balestra et al. Phys. Rev. Lett., 81:4572-4575, 1998.

[128] P. Moskal et al. J. Phys., G29:2235-2246, 2003.

[129] W. Brodowski et al. Phys. Rev. Lett., 88:192301, 2002.

[130] J. Patzold et al. nucl-ex/0301019, 2003.

[131] J. Johanson et al. Nucl. Phys., A712:75-94, 2002.

[132] C. Quentmeier et al. Phys. Lett., B515:276-282, 2001.

[133] V. Kleber et al. nucl-ex/0304020, 2003.

[134] H. O. Meyer and P. Schwandt (eds.). Nuclear physics at storage rings. proceedings, 4th international conference, stori'99, bloomington, usa, september 12-16, 1999.

[135] H. E. Conzett. Phys. Rev., C48:423-428, 1993.

[136] F. Hinterberger et al. nucl-ex/9810003, 1998.

[137] H. Leutwyler. Phys. Lett., B378:313-318, 1996.

[138] B. Kerbikov and F. Tabakin. Phys. Rev., C62:064601, 2000.

[139] J. A. Niskanen and M. Vestama. Phys. Lett., B394:253-259, 1997.

[140] E. J. Stephenson et al. nucl-ex/0305032, 2003.

[141] U. van Kolck, J. A. Niskanen, and G. A. Miller. Phys. Lett., B493:65-72, 2000.

[142] A. Gardestig et al. nucl-th/0402021.

[143] A. E. Kudryavtsev and V. E. Tarasov. JETP Lett., 72:410-414, 2000. 
[144] V. Yu. Grishina, L. A. Kondratyuk, M. Buscher, W. Cassing, and H. Stroher. Phys. Lett., B521:217-224, 2001.

[145] A. E. Kudryavtsev, V. E. Tarasov, J. Haidenbauer, C. Hanhart, and J. Speth. nucl-th/0304052, 2003.

[146] K. Hagiwara et al. Phys. Rev. D, 66:010001, 2002.

[147] A. E. Woodruf. Phys. Rev., 117:1113, 1960.

[148] J. Niskanen. Phys. Lett., B289:227, 1992.

[149] T.-S. Lee and D. Riska. Phys. Rev. Lett., 70:2237, 1993.

[150] C. J. Horowitz, H. O. Meyer, and D. K. Griegel. Phys. Rev. C, 49:1337, 1994.

[151] F. Hachenberg and H. J. Pirner. Ann. Phys., 112:401, 1978.

[152] E. Gedalin, A. Moalem, and L. Rasdolskaya. Nucl. Phys., A652:287-307, 1999.

[153] C. Hanhart, J. Haidenbauer, A. Reuber, C. Schütz, and J. Speth. Phys. Lett. $B, 358: 21,1995$.

[154] A. Engel, A. K. Dutt-Mazumder, R. Shyam, and U. Mosel. Nucl. Phys., A603:387-414, 1996.

[155] V. Bernard, N. Kaiser, and U.-G. Meißner. Eur. Phys. J., A4:259-275, 1999.

[156] J. Adam, A. Stadler, M. T. Pena, and F. Gross. Phys. Lett. B, 407:97, 1997.

[157] G. H. Martinus, O. Scholten, and J. A. Tjon. Phys. Rev., C56:2945-2962, 1997.

[158] M. D. Cozma, G. H. Martinus, O. Scholten, R. G. E. Timmermans, and J. A. Tjon. Phys. Rev., C65:024001, 2002.

[159] U. van Kolck, G. A. Miller, and D. O. Riska. Phys. Lett. B, 388:679, 1996.

[160] M. T. Pena, D. O. Riska, and A. Stadler. Phys. Rev., C60:045201, 1999.

[161] E. Hernandez and E. Oset. Phys. Rev., C60:025204, 1999.

[162] B. Y. Park, F. Myhrer, J. R. Morones, T. Meißner, and K. Kubodera. Phys. Rev. C, 53:1519, 1996.

[163] T. D. Cohen, J. L. Friar, G. A. Miller, and U. van Kolck. Phys. Rev. C, 53:2661, 1996. 
[164] C. Hanhart, J. Haidenbauer, M. Hoffmann, U.-G. Meißner, and J. Speth. Phys. Lett., B424:8-14, 1998.

[165] C. da Rocha, G. Miller, and U. van Kolck. Phys. Rev., C61:034613, 2000.

[166] E. Gedalin, A. Moalem, and L. Razdolskaya. AIP Conf. Proc., 603:247-248, 2001.

[167] S. R. Beane, V. Bernard, E. Epelbaum, U.-G. Meißner, and D. R. Phillips. Nucl. Phys., A720:399-415, 2003.

[168] S. Weinberg. Phys. Rev. Lett., 17:616, 1966.

[169] Y. Tomozawa. Nuovo Cimento, 46A:707, 1966.

[170] V. Bernard, N. Kaiser, and U.-G. Meißner. Nucl. Phys., B457:147, 1995.

[171] V. Bernard, N. Kaiser, and U.-G. Meißner. Nucl. Phys., B457:147-174, 1995.

[172] V. Bernard, N. Kaiser, and U.-G. Meißner. Nucl. Phys., A615:483-500, 1997.

[173] T. Sato, T. S. H. Lee, F. Myhrer, and K. Kubodera. Phys. Rev. C, 56:1246, 1997.

[174] C. Hanhart, G. A. Miller, F. Myhrer, T. Sato, and U. van Kolck. Phys. Rev., C63:044002, 2001.

[175] J. Haidenbauer, K. Holinde, and M. B. Johnson. Phys. Rev. C, 48:2190, 1993.

[176] C. Schütz, J. Haidenbauer, and K. Holinde. Phys. Rev. C, 54:1561, 1996.

[177] D. V. Bugg and R. A.Bryon. Nucl. Phys., A540:449, 1992.

[178] M. B. Johnson. Ann. Phys., 97:400, 1976.

[179] C. Schütz, J. W. Durso, K. Holinde, and J. Speth. Phys. Rev. C, 49:2671, 1994.

[180] O. Krehl, C. Hanhart, S. Krewald, and J. Speth. Phys. Rev., C60:055206, 1999.

[181] K. Kawarabayashi and M. Suzuki. Phys. Rev. Lett., 16:255, 1966.

[182] Riazuddin and Fayyazuddin. Phys. Rev., 147:1071-1073, 1966.

[183] G. Höhler. Handbook of Pion-Nucleon Scattering. Fachinformationszentrum Karlsruhe, Karlsruhe, 1979. 
[184] R. A. Arndt, J. M. Ford, and L. D. Roper. Phys. Rev. D, 32:1085, 1985.

[185] T. Ericson and W. Weise. Pions and Nuclei. Clarendon Press, Oxford, 1988.

[186] S. Weinberg. Phys. Lett. B, 251:288, 1990.

[187] M. E. Schillaci, R. R. Silbar, and J. E. Young. Phys. Rev., 179:1539, 1969.

[188] D.S. Bender. Nuovo Cimento, 56A:625, 1968.

[189] R. Baier and H. Kühnelt. Nuovo Cimento, 63A:135, 1969.

[190] S.L. Adler and Y. Dothan. Phys. Rev., 151:1267, 1966.

[191] F.E. Low. Phys. Rev., 110:1958, 1958.

[192] A. Dobado, A. Gomez-Nicola, A.L. Maroto, and J.R. Pelaez. Effective Lagrangians for the Standard Model. Springer-Verlag, Berlin, 1997.

[193] C. Hanhart, D. R. Phillips, and S. Reddy. Phys. Lett., B499:9-15, 2001.

[194] S. Weinberg. Physica, A96:327, 1979.

[195] J. Gasser and H. Leutwyler. Ann. Phys., 158:142, 1984.

[196] J. Bijnens, G. Colangelo, G. Ecker, J. Gasser, and M. E. Sainio. Nucl. Phys., B508:263-310, 1997.

[197] V. Bernard, N. Kaiser, and U.-G. Meißner. Int. J. Mod. Phys., E4:193-346, 1995.

[198] C. Ordonez, L. Ray, and U. van Kolck. Phys. Rev. Lett., 72:1982-1985, 1994.

[199] C. Ordonez, L. Ray, and U. van Kolck. Phys. Rev., C53:2086-2105, 1996.

[200] E. Epelbaum, W. Glockle, and U.-G. Meißner. Nucl. Phys., A637:107-134, 1998.

[201] E. Epelbaum, W. Glockle, and U.-G. Meißner. Nucl. Phys., A671:295-331, 2000.

[202] T. R. Hemmert, B. R. Holstein, and J. Kambor. Phys. Lett., B395:89-95, 1997.

[203] C. Hanhart, U. van Kolck, and G. A. Miller. Phys. Rev. Lett., 85:2905-2908, 2000 .

[204] C. Hanhart and N. Kaiser. Phys. Rev., C66:054005, 2002. 
[205] V. Dmitrasinovic, K. Kubodera, F. Myhrer, and T. Sato. Phys. Lett., B465:4354, 1999.

[206] S. Ando, T. Park, and D.-P. Min. Phys. Lett., B509:253-262, 2001.

[207] E. Gedalin, A. Moalem, and L. Razdolskaya. AIP Conf. Proc., 603:247-248, 2001.

[208] U. van Kolck. Phys. Rev., C49:2932-2941, 1994.

[209] N. Fettes, U.-G. Meissner, and Sven Steininger. Nucl. Phys., A640:199-234, 1998.

[210] N. Fettes and U.-G. Meissner. Nucl. Phys., A676:311, 2000.

[211] P. Buttiker and U.-G. Meissner. Nucl. Phys., A668:97-112, 2000.

[212] N. Fettes and U.-G. Meissner. Nucl. Phys., A679:629-670, 2001.

[213] U. van Kolck. Prog. Part. Nucl. Phys., 43:337-418, 1999.

[214] M.F.M. Lutz. Nucl. Phys., A677:241-312, 2000.

[215] E. Epelbaum et al. Phys. Rev., C66:064001, 2002.

[216] S. Weinberg. Phys. Lett., B295:114-121, 1992.

[217] M. Gell-Mann, R. J. Oakes, and B. Renner. Phys. Rev., 175:2195-2199, 1968.

[218] V. Bernard, N. Kaiser, J. Gasser, and U.-G. Meißner. Phys. Lett., B268:291$295,1991$.

[219] R. W. Flammang et al. Phys. Rev., C58:916-931, 1998.

[220] D. Huber, J. L. Friar, A. Nogga, H. Witala, and U. van Kolck. Few Body Syst., 30:95-120, 2001.

[221] A. W. Thomas and R. H. Landau. Phys. Rept., 58:121, 1980.

[222] T. E. O. Ericson, B. Loiseau, and A. W. Thomas. Phys. Rev., C66:014005, 2002.

[223] V. Baru, J. Haidenbauer, C. Hanhart, and J. A. Niskanen. Eur. Phys. J., A16:437-446, 2003.

[224] R. Haag. Phys. Rev., 112:669, 1958. 
[225] H. Lehmann, K. Symanzik, and W. Zimmermann. Nuovo Cim., 1:205-225, 1955.

[226] H. W. Fearing. Phys. Rev. Lett., 81:758-761, 1998.

[227] H. W. Fearing and S. Scherer. Phys. Rev., C62:034003, 2000.

[228] E. Oset, H. Toki, M. Mizobe, and T. T. Takahashi. Prog. Theor. Phys., 103:351-365, 2000.

[229] G. Janssen, J. W. Durso, K. Holinde, B. C. Pearce, and J. Speth. Phys. Rev. Lett., 71:1978-1981, 1993.

[230] R. Bockmann, C. Hanhart, O. Krehl, S. Krewald, and J. Speth. Phys. Rev., C60:055212, 1999.

[231] M. Schwamb and H. Arenhovel. Nucl. Phys., A690:682-710, 2001.

[232] A. Motzke, C. Elster, and C. Hanhart. Phys. Rev., C66:054002, 2002.

[233] O. Krehl, C. Hanhart, S. Krewald, and J. Speth. Phys. Rev., C62:025207, 2000 .

[234] N. Kaiser. Phys. Rev., C60:057001, 1999.

[235] N. Kaiser. Eur. Phys. J., A5:105-110, 1999.

[236] K. Nakayama, A. Szczurek, C. Hanhart, J. Haidenbauer, and J. Speth. Phys. Rev., C57:1580-1587, 1998.

[237] K. Nakayama, J. Haidenbauer, and J. Speth. Phys. Rev., C63:015201, 2001.

[238] K. Nakayama, J. W. Durso, J. Haidenbauer, C. Hanhart, and J. Speth. Phys. Rev., C60:055209, 1999.

[239] K. Nakayama, J. Speth, and T. S. H. Lee. Phys. Rev., C65:045210, 2002.

[240] K. Nakayama, J. Haidenbauer, C. Hanhart, and J. Speth. Phys. Rev., C68:045201, 2003.

[241] K. Nakayama, H. F. Arellano, J. W. Durso, and J. Speth. Phys. Rev., C61:024001, 2000.

[242] M. Gell-Mann. Caltech Report CTSL-20 (1961).

[243] S. Okubo. Prog. Theor. Phys., 27:949, 1962. 
[244] N. Kaiser, P. B. Siegel, and W. Weise. Phys. Lett., B362:23-28, 1995.

[245] T. Inoue, E. Oset, and M. J. Vicente Vacas. Phys. Rev., C65:035204, 2002.

[246] C. Schutz, J. Haidenbauer, J. Speth, and J. W. Durso. Phys. Rev., C57:1464$1477,1998$.

[247] U. Loring, B. C. Metsch, and H. R. Petry. Eur. Phys. J., A10:395-446, 2001.

[248] H. Garcilazo, M. T. Pena, and ESFM Mexico. Phys. Rev., C66:034606, 2002.

[249] M. T. Pena, H. Garcilazo, and D. O. Riska. Nucl. Phys., A683:322-338, 2001.

[250] E. Gedalin, A. Moalem, and L. Razdolskaja. Nucl. Phys., A634:368-392, 1998.

[251] A. Moalem, E. Gedalin, L. Razdolskaya, and Z. Shorer. Nucl. Phys., A589:649659, 1995.

[252] A. Moalem, E. Gedalin, L. Razdolskaya, and Z. Shorer. Nucl. Phys., A600:445460, 1996.

[253] A. B. Santra and B. K. Jain. Phys. Rev., C64:025201, 2001.

[254] J. F. Germond and C. Wilkin. Nucl. Phys., A518:308-316, 1990.

[255] Goran Faldt and Colin Wilkin. Phys. Scripta, 64:427-438, 2001.

[256] T. Vetter, A. Engel, T. Biro, and U. Mosel. Phys. Lett., B263:153-156, 1991.

[257] N. I. Kochelev, V. Vento, and A. V. Vinnikov. Phys. Lett., B472:247-252, 2000.

[258] V. Yu. Grishina et al. Phys. Lett., B475:9-16, 2000.

[259] M. Batinic, A. Svarc, and T. S. H. Lee. Phys. Scripta, 56:321-324, 1997.

[260] A. Sibirtsev et al. Phys. Rev., C65:044007, 2002.

[261] A. Fix and H. Arenhoevel. arXiv:nucl-th/0310034.

[262] Andrzej Deloff. arXiv:nucl-th/0309059.

[263] F. Rathmann. in Hadron physics at COSY, A. Gillitzer et al. (eds.), arXiv:hep$\mathrm{ph} / 0311071$.

[264] B. Sechi-Zorn et al. Phys. Rev., 175:1735, 1968.

[265] F. Eisele et al. Phys. Lett. B, 37:204, 1971. 
[266] G. Alexander et al. Phys. Rev., 173:1452, 1968.

[267] R. Siebert et al. Nucl. Phys., A567:819-843, 1994.

[268] P. M. M. Maessen, T. A. Rijken, and J. J. de Swart. Phys. Rev. C, 40:2226, 1989.

[269] T. A. Rijken, V. G. J. Stoks, and Y. Yamamoto. Phys. Rev., C59:21-40, 1999.

[270] B. Holzenkamp, K. Holinde, and J. Speth. Nucl. Phys., A500:485, 1989.

[271] V. Mull, J. Haidenbauer, T. Hippchen, and K. Holinde. Phys. Rev. C, 44:1337, 1991.

[272] U.-G. Meißner. Phys. Scripta, T99:68-83, 2002.

[273] A. Nogga, H. Kamada, and W. Glockle. Phys. Rev. Lett., 88:172501, 2002.

[274] J. A. Pons, S. Reddy, M. Prakash, J. M. Lattimer, and J. A. Miralles. Astrophys. J., 513:780, 1999.

[275] A. V. Anisovich, V. V. Anisovich, and V. A. Nikonov. Eur. Phys. J., A12:103$115,2001$.

[276] R.L. Jaffe. Phys. Rev. D, 15:281, 1977.

[277] N. N. Achasov. Phys. Atom. Nucl., 65:546-551, 2002.

[278] J. D. Weinstein and N. Isgur. Phys. Rev., D41:2236, 1990.

[279] G. Janssen, B. C. Pearce, K. Holinde, and J. Speth. Phys. Rev., D52:2690$2700,1995$.

[280] J. A. Oller and E. Oset. Nucl. Phys., A620:438-456, 1997.

[281] F. E. Close and N. A. Tornqvist. J. Phys., G28:R249-R267, 2002.

[282] B. Aubert et al. Phys. Rev. Lett., 90:242001, 2003.

[283] E. van Beveren and G. Rupp. Phys. Rev. Lett., 91:012003, 2003.

[284] S.A. Devyanin N.N. Achasov and G.N. Shestakov. Phys. Lett., B88:367-371, December 1979.

[285] J. Gasser and H. Leutwyler. Phys. Rept., 87:77-169, 1982.

[286] O. Krehl, R. Rapp, and J. Speth. Phys. Lett., B390:23-28, 1997. 
[287] S. Weinberg. Phys. Rev., 130:776, 1963.

[288] V. Baru, J. Haidenbauer, C. Hanhart, Yu. Kalashnikova, and A. Kudryavtsev. arXiv:hep-ph/0308129.

[289] P. Weber et al. Nucl. Phys., A501:765, 1989.

[290] S. MayTal-Beck et al. Phys. Rev. Lett., 68:3012, 1992.

[291] S. Schneider, J. Haidenbauer, C. Hanhart, and J. A. Niskanen. Phys. Rev., C67:044003, 2003.

[292] J. Niskanen. Phys. Rev. C, 44:2222, 1991.

[293] V. N. Nikulin et al. Phys. Rev., C54:1732-1740, 1996.

[294] R. Wurzinger et al. Phys. Rev., C51:443-446, 1995.

[295] J. Berger et al. Phys. Rev. Lett., 61:919-922, 1988.

[296] B. Mayer et al. Phys. Rev., C53:2068-2074, 1996.

[297] R. Wurzinger et al. Phys. Lett., B374:283-288, 1996.

[298] R. Frascaria et al. Phys. Rev., C50:537-540, 1994.

[299] J. F. Germond and C. Wilkin. J. Phys., G14:181-190, 1988.

[300] J. M. Laget and J. F. Lecolley. Phys. Rev. Lett., 61:2069-2072, 1988.

[301] J. F. Germond and C. Wilkin. J. Phys., G15:437, 1989.

[302] G. Faeldt and C. Wilkin. Nucl. Phys., A587:769-786, 1995.

[303] K. P. Khemchandani, N. G. Kelkar, and B. K. Jain. Nucl. Phys., A708:312$324,2002$.

[304] K. Kilian and H. Nann. in: Meson production near threshold, eds. H. Nann and E.J. Stephenson, AIP Conf. Proc. No. 221 (AIP, New York, 1990) p. 185.

[305] S.Ahmad et al. Physics at LEAR with low-energy antiprotons (eds. Amsler et al., Harwood Academic, New York 1987) 447.

[306] Y. S. Golubeva, W. Cassing, L. A. Kondratyuk, A. Sibirtsev, and M. Buscher. Eur. Phys. J., A7:271-277, 2000.

[307] R. Schleichert. persönliche Mitteilung. 
[308] M. Buscher, F. P. Sassen, N. N. Achasov, and L. Kondratyuk. Investigation of light scalar resonances at cosy. hep-ph/0301126, 2003.

[309] R. Sindak et al. The hires experiment at cosy and first test of a new cherenkov detector. Prepared for Meson 2002: 7th International Workshop on Meson Production, Properties and Interaction, Cracow, Poland, 24- 28 May 2002.

[310] A.R. Edmonds. Angular momentum in Quantum Mechanics. Princeton University Press, Princeton, NJ, 1957.

[311] D. B. Kaplan, M. J. Savage, and M. B. Wise. Phys. Lett., B424:390-396, 1998. 